\title{
Principles of X-ray Navigation
}

\author{
John Eric Hanson \\ Stanford Linear Accelerator Center \\ Stanford University \\ Stanford, CA 94309
}

SLAC-Report-809

Prepared for the Department of Energy

under contract number DE-AC02-76SF00515

Printed in the United States of America. Available from the National Technical Information Service, U.S. Department of Commerce, 5285 Port Royal Road, Springfield, VA 22161. 
This document, and the material and data contained therein, was developed under sponsorship of the United States Government. Neither the United States nor the Department of Energy, nor the Leland Stanford Junior University, nor their employees, nor their respective contractors, subcontractors, or their employees, makes an warranty, express or implied, or assumes any liability of responsibility for accuracy, completeness or usefulness of any information, apparatus, product or process disclosed, or represents that its use will not infringe privately owned rights. Mention of any product, its manufacturer, or suppliers shall not, nor is it intended to, imply approval, disapproval, or fitness of any particular use. A royalty-free, nonexclusive right to use and disseminate same of whatsoever, is expressly reserved to the United States and the University. 


\title{
Principles of X-ray Navigation
}

\author{
John Eric Hanson \\ Stanford Linear Accelerator Center \\ Stanford University \\ Stanford, CA 94309
}

SLAC-Report-809

Prepared for the Department of Energy

under contract number DE-AC02-76SF00515

Printed in the United States of America. Available from the National Technical Information Service, U.S. Department of Commerce, 5285 Port Royal Road, Springfield, VA 22161. 


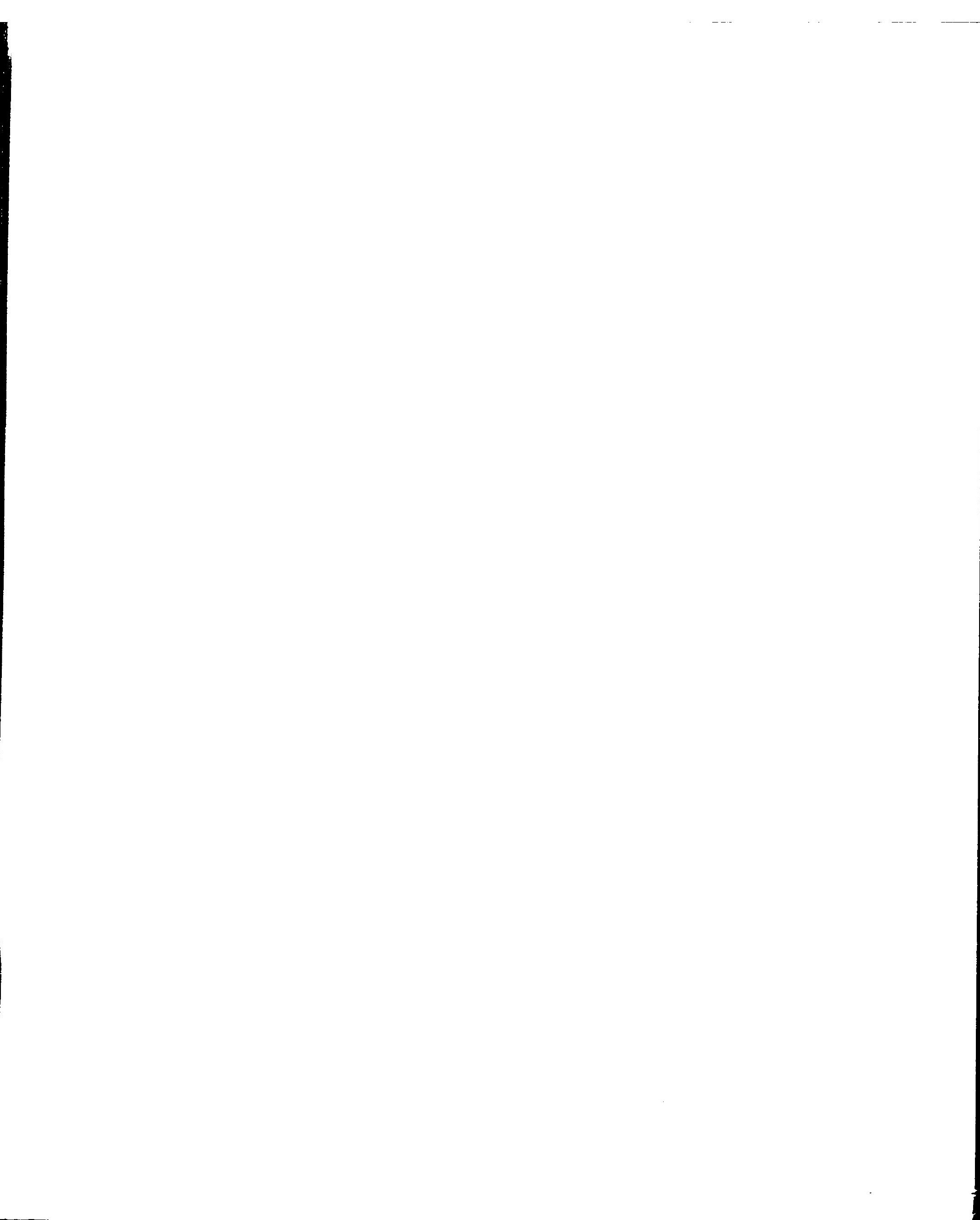


PRINCIPLES OF X-RAY NAVIGATION

\author{
A DISSERTATION SUBMTTED TO THE \\ DEPARTMENT OF AERONAUTICS AND ASTRONAUTICS \\ AND THE COMMITTEE ON GRADUATE STUDIES \\ OF STANFORD UNIVERSITY \\ IN PARTIAL FULFILLMENT OF THE REQUIREMENTS \\ FOR THE DEGREE OF \\ DOCTOR OF PHILOSOPHY
}

By

John Eric Hanson

March, 1996 
() Copyright 1996

by

John E. Hanson 


\begin{abstract}
$\mathrm{X}$-ray navigation is a new concept in satellite navigation in which orientation, position and time are measured by observing stellar emissions in $\mathrm{X}$-ray wavelengths. $\mathrm{X}$ ray navigation offers the opportunity for a single instrument to be used to measure these parameters autonomously. Furthermore, this concept is not limited to missions in close proximity to the earth. X-ray navigation can be used on a variety of missions from satellites in low earth orbit to spacecraft on interplanetary missions.

In 1997 the Unconventional Stellar Aspect Experiment (USA) will be launched as part of the Advanced Research and Global Observation Satellite (ARGOS). USA will provide the first platform for real-time experimentation in the field of $x$-ray navigation and also serves as an excellent case study for the design and manufacturing of space qualified systems in small, autonomous groups.

Current techniques for determining the orientation of a satellite rely on observations of the earth, sun and stars in infrared, visible or ultraviolet wavelengths. It is possible to use $x$-ray imaging devices to provide arcsecond level measurement of attitude based on star pattems in the $x$-ray sky. This technique is explored with a simple simulation.
\end{abstract}


Collimated $\mathrm{x}$-ray detectors can be used on spinning satellites to provide a cheap and reliable measure of orientation. This is demonstrated using observations of the Crab Pulsar taken by the High Energy Astronomy Observatory (HEAO-1) in 1977. A single instrument concept is shown to be effective, but dependent on an a priori estimate of the guide star intensity and thus susceptible to errors in that estimate. A star scanner based on a differential measurement from two $x$-ray detectors eliminates the need for an a priori estimate of the guide star intensity. A first order model and a second order model of the two star scanner concepts are considered.

Many of the stars that emit in the $x$-ray regime are also $x$-ray pulsars with frequency stability approaching a part in $10^{9}$. By observing these pulsations, a satellite can keep accurate time autonomously. We have demonstrated the acquisition and tracking of the Crab nebula pulsar by simulating the operation of a phase-locked loop. 


\section{Acknowledgments}

As I put the finishing touches on this dissertation, I should take a moment to thank the myriad of people who have made this possible.

First, I would like to thank my two advisors, Professor Dan DeBra and Professor Elliott Bloom, for their guidance and understanding during my stay here. I have been fortunate to work with both of their groups simultaneously and have learned more about engineering in the past three years than I ever thought possible. I thank Chris Lages and Han Wen both for their friendship and for the endless and far ranging discussions - some about work and some not.

I want to thank Professor Steve Rock for serving on my oral committee and my reading committee with Professor DeBra and Professor Bloom, and Professor Peter Michelson for chairing my oral committee. Their insights and suggestions have improved this work immeasurably.

I want to thank all of the members of the USA team for their friendship and help these past three years - Gary, John, Andrew and Linda Lee. I have enjoyed working with the entire USA team (SLAC and NRL contingents) immensely. The USA experiment and this thesis certainly would not have been possible without the tireless efforts of Dr. Kent Wood and Dr. Michael Lovellette and to them both I am grateful. I also want to thank Dr. Wood for allowing me to base this thesis on some of his original ideas for $\mathrm{x}$-ray navigation.

Much of this dissertation is made possible by the availability of data from the HEAO-Al mission. This has been maintained by Dr. Lynn Cominsky, Han Wen and Andrew Lee. I thank them all for their help in both getting the data I needed and in understanding it.

I would like to thank my parents, Jack and Judy Hanson, and my sisters Lori and Lisa for their support and encouragement over the past six years. Contrary to popular belief, life at Stanford is not always beautiful weather and wonderful scenery. It helps to 
have family and friends close by to take the edge off. To this end, I have been blessed with many old and new friends from the east coast to the west coast. Darren, Audrey and Kara have kept me generally sane, moderately cultured and occasionally well fed. Mark, Rob, Ed, Theresa, Jerry and Steve added much needed humor with every e-mail and were there for some of our less cultured (but nonetheless important) endeavors.

And last, but certainly not least, I want to thank all of the new friends I have made at Stanford (the aforementioned Chris, and the here-mentioned Bob, Steve, Phil, Ed and Susan) for a wonderful six years. 


\section{Table of Contents}

Abstract iv

Acknowledgments

1. Introduction 1

1.1 Problem Statement 1

1.2 History of X-ray Missions 2

$\begin{array}{lll}1.3 & \text { Satellite Navigation Principles } & 6\end{array}$

1.3.1 Attitude Determination 6

1.3.2 Time Keeping 8

1.3.3 Position Determination 9

1.4 USA Experiment Design Issues 10

$\begin{array}{lll}1.5 & \text { USA/HEAO Navigation Experiments } & 10\end{array}$

1.6 Contributions 11

$\begin{array}{lll}1.7 & \text { Thesis Outline } & 12\end{array}$

2. Design Aspects of the Unconventional Stellar Aspect Experiment 15

$\begin{array}{lll}2.1 & \text { USA Mission } & 15\end{array}$

$\begin{array}{lll}2.2 & \text { USA General Description } & 17\end{array}$

2.3 USA Structural Design and Analysis 22

2.4 USA Structural Test Program 28

2.5 USA Thermal Analysis 29 
3. X-ray Attitude Determination 38

3.1 Introduction 38

3.2 X-ray Star Mappers on Pointed Satellites 39

3.2.1 Missions and Methodologies $\quad 39$

3.2.2 Pointing Feasibility Study 41

3.3 X-ray Star Scanners on Spinning Spacecraft 49

$\begin{array}{ll}\text { 3.3.1 Instrument Fundamentals } & 50\end{array}$

3.3.2 General Star Scanner Description 53

3.3.3 X-ray Spin Rate Sensor $\quad 57$

3.3.4 Single Collimator X-ray Star Scanner 58

$\begin{array}{lll}\text { 3.3.5 Differential Collimator X-ray Star Scanner } & 60\end{array}$

3.3.6 Second Order Star Scanner Model 64

3.3.6.1 Rotation about Spin Axis 66

3.3.6.2 Rotation in Pitch Direction 66

3.3.6.3 Rotation about Instrument Line of Sight 66

$\begin{array}{lll}\text { 3.3.6.4 Polhode Motion } & 67\end{array}$

4. HEAO-A1 Attitude Determination Studies 68

$\begin{array}{lll}4.1 & \text { Introduction } & 68\end{array}$

4.2 HEAO-A1 Description $\quad 68$

4.3 HEAO-A1 Module 3 Attitude Determination Algorithm 72

4.4 HEAO-Al Module 3/5 Attitude Determination Algorithm 83

4.5 HEAO-A1 Module 3/5 with Second Order Model 91

4.6 HEAO-A1 Attitude Determination Summary 96 
5. Autonomous X-ray Time Keeping 97

$\begin{array}{lll}5.1 & \text { Introduction } & 97\end{array}$

5.2 Phase Locked Loops 98

$\begin{array}{lll}5.3 & \text { Error Sources } & 103\end{array}$

$\begin{array}{lll}5.4 & \text { Simulation Description } & 106\end{array}$

$\begin{array}{ll}\text { 5.5 Simulated Phase Locked Loop Design } & 110\end{array}$

$\begin{array}{lll}5.6 & \text { Simulation Results } & 112\end{array}$

6. Conclusions and Recommendations 116

$\begin{array}{lll}6.1 & \text { Conclusions } & 116\end{array}$

$\begin{array}{lll}6.2 & \text { Recommendations for Future Research } & 118\end{array}$

$\begin{array}{ll}\text { Appendices } & 120\end{array}$

A. Unconventional Stellar Aspect Experiment 120 Interface Control Document

B. Selected Astrogravity Notes 139

C. Pointing Feasibility Study - C Procedures 156

D. Derivation of Equations Describing Collimator Performance 163

$\begin{array}{ll}\text { E. First Order Star Scanner Model } & 166\end{array}$

F. Second Order Star Scanner Model $\quad 178$

G. HEAO-A1 Studies - IDL Procedures 184 
H. Phase Locked Loops

I. Phase Locked Loop Simulation - IDL Procedures

204

References

208 


\section{List of Figures}

2.1 Advanced Research and Global Observation Satellite 16

2.2 Advanced Research and Global Observation Satellite Orbit 16

2.3 Unconventional Stellar Aspect Experiment 19

$\begin{array}{lll}2.4 & \text { USA Experiment Proportional Chamber } & 19\end{array}$

2.5 USA Experiment Pylon/Baseplate Assembly 21

2.6 USA Experiment Finite Element Model 24

2.7 USA Experiment Yoke Assembly 26

2.8 USA Experiment Pylon Assembly 26

2.9 USA Experiment Thermal Control System 30

2.10 USA Experiment Pylon/Baseplate Thermal Model 32

2.11 USA Experiment Detector Thermal Model 32

2.12 USA Experiment Yoke Thermal Model 33

2.13a USA Experiment Operational Mode 37

2.13b USA Experiment Sunsafe Mode $\quad 37$

3.1 HEAO-A1 X-ray Sky Map 43

3.2 X-ray Star Mapper Attitude Determination Algorithm 45

3.3 Ideal Star Mapper Sensitivity 47

3.4 Ideal Star Mapper Exposure Time 48

3.5 Two Dimensional Collimator Geometry 51

3.6 Ideal Two Dimensional Collimator Response 51

3.7 Ideal Three Dimensional Collimator Response 53

3.8a Single Collimator X-ray Star Scanner Geometry 58

3.8b Single Collimator X-ray Star Scanner Response 59

3.9a Dual Collimator/Dual Detector X-ray Star Scanner Geometry 61

3.9b Dual Collimator/Dual Detector X-ray Star Scanner Response 61

3.10a Dual Collimator/Single Detector X-ray Star Scanner Geometry 62

3.10b Dual Collimator/Single Detector X-ray Star Scanner Response 62 
4.1 HEAO-AI LASS Mission $\quad 69$

4.2 Sample HEAO-A1 Module 3 Data 75

4.3 Single Detector Fit Parameters $\quad 77$

4.4 Single Detector Attitude Study Results 81

4.5 HEAO-A1 Differential Detector Ambiguity 84

4.6 Sample HEAO-A1 Module 3 and Module 5 Data 86

4.7 Dual Detector Fit Parameters 88

4.8 Dual Detector Attitude Study Results 90

4.9 Dual Detector/Second Order Model Fit Parameters 92

4.10 Dual Detector/Second Order Model Attitude Study Results 95

5.1 Phase Locked Loop Schematic 99

5.2 Observed Crab Nebula Pulsar Pulse Shape 109

5.3 Modeled Crab Nebula Pulsar Pulse Shape 109

5.4 Phase Locked Loop Simulated Performance 113

5.5 Phase Locked Loop Simulated Signal Comparison 114

5.6 Phase Locked Loop Simulated Performance (Zero Pulsar Input) 115

$\begin{array}{ll}\text { D.1 Collimator Angle Definitions } & 165\end{array}$

E.1 Transformation from Celestial to Guide Star Coordinate System 176

E.2 Transformation from Guide Star to Spacecraft Coordinate System 176

E.3 Transformation from Spacecraft to Instrument Coordinate System 177

E.4 Guide Star Representation in the Instrument Coordinate System 177 


\section{List of Tables}

$\begin{array}{lll}2.1 & \text { USA Orbit Parameters } & 17\end{array}$

2.2 USA Mass Properties 20

2.3 USA ICD Static Flight Accelerations 23

2.4 USA Design Flight Accelerations 23

2.5 USA Thermal Design 30

3.1 Ideal Detector Parameters 42

$\begin{array}{lll}4.1 & \text { HEAO-A1 Module Properties } & 70\end{array}$

$\begin{array}{lll}5.1 & \text { PLL Characteristics } & 103\end{array}$

$\begin{array}{lll}5.2 & \text { Pulsar Characteristics } & 107\end{array}$ 


\section{List of Abbreviations}

\begin{tabular}{|c|c|}
\hline ADACS & Attitude Determination and Control System \\
\hline $\mathrm{ADB}$ & Aluminum Dip Braze Corporation \\
\hline ARGOS & Advanced Research and Global Observation Satellite \\
\hline $\mathrm{CAD}$ & Computer Aided Design \\
\hline CAEDS & Computer Aided Engineering and Design Software \\
\hline CE Box & Central Electronics Box \\
\hline CXE & Cosmic X-ray Experiment \\
\hline FEM & Finite Element Model \\
\hline FOS & Factor of Safety \\
\hline FOV & Field of View \\
\hline GP-A & Gravity Probe A \\
\hline GPS & Global Positioning System \\
\hline HEAO-1 & High Energy Astronomical Observatory 1 \\
\hline HEAO-Al & High Energy Astronomical Observatory 1, Experiment A \\
\hline ICD & Interface Control Document \\
\hline IDL & Interactive Data Language \\
\hline LASS & Large Area Sky Survey \\
\hline LEO & Low Earth Orbit \\
\hline MC & Modulation Collimator \\
\hline Mod 3 & Module 3 of HEAO-A1 \\
\hline Mod 5 & Module 5 of HEAO-A1 \\
\hline MOS & Margin of Safety \\
\hline NRL & Naval Research Laboratory \\
\hline OSO-3 & Orbiting Solar Observatory 3 \\
\hline PLL & Phase Locked Loop \\
\hline
\end{tabular}


SIXI

SLAC

SNR

USA

$\mathrm{VCO}$
Silicon Imaging $\mathrm{X}$-ray Instrument

Stanford Linear Accelerator Center

Signal to Noise Ratio

Unconventional Stellar Aspect Experiment

Voltage Controlled Oscillator 


\title{
List of Symbols
}

\author{
Pointing Feasibility Study \\ $t_{0} \quad$ time of initial attitude estimate \\ $t_{1} \quad$ time of updated attitude estimate \\ $\Delta \mathrm{t} \quad \mathrm{x}$-ray star mapper sample period \\ te $\quad x$-ray star mapper exposure time \\ $X \quad$ number of photons from bin containing $x$-ray background \\ $Y_{i} \quad$ number of photons from bin containing $x$-ray guide star \\ $\mu_{\mathrm{s}} \quad$ mean guide star count rate (counts/sec*m2) \\ A instrument effective collecting area \\ $\mathrm{P}(\mathrm{S}) \quad$ probability of successful attitude update \\ $\mu_{\mathrm{b}} \quad$ mean background count rate (counts/sec${ }^{*} \mathrm{~m} 2$ ) \\ X-ray Star Scanners - Instrument Fundamentals \\ $\alpha \quad \mathrm{x}$-ray photon angle of incidence - one dimensional \\ $\mathrm{L}_{0} \quad$ geometric length of detector \\ $\mathrm{L} \quad$ effective length of detector \\ $\mathrm{T}_{\mathrm{c}} \quad$ collimator transmission \\ $\alpha_{0} \quad$ collimator half-cone angle - one dimensional \\ $h_{0} \quad$ collimator height \\ $\rho \quad x$-ray photon angle of incidence - two dimensional \\ $\rho_{0} \quad$ collimator half-cone angle - two dimensional
}

X-ray Star Scanner Models

$\left(\mathrm{X}_{\mathrm{c}}, \mathrm{Y}_{\mathrm{c}}, \mathrm{Z}_{\mathrm{c}}\right) \quad$ celestial coordinate system basis

RA right ascension of guide star in celestial coordinates

DEC declination of guide star in celestial coordinates

$(\mathrm{X}, \mathrm{Y}, \mathrm{Z}) \quad$ guide star coordinate system basis 


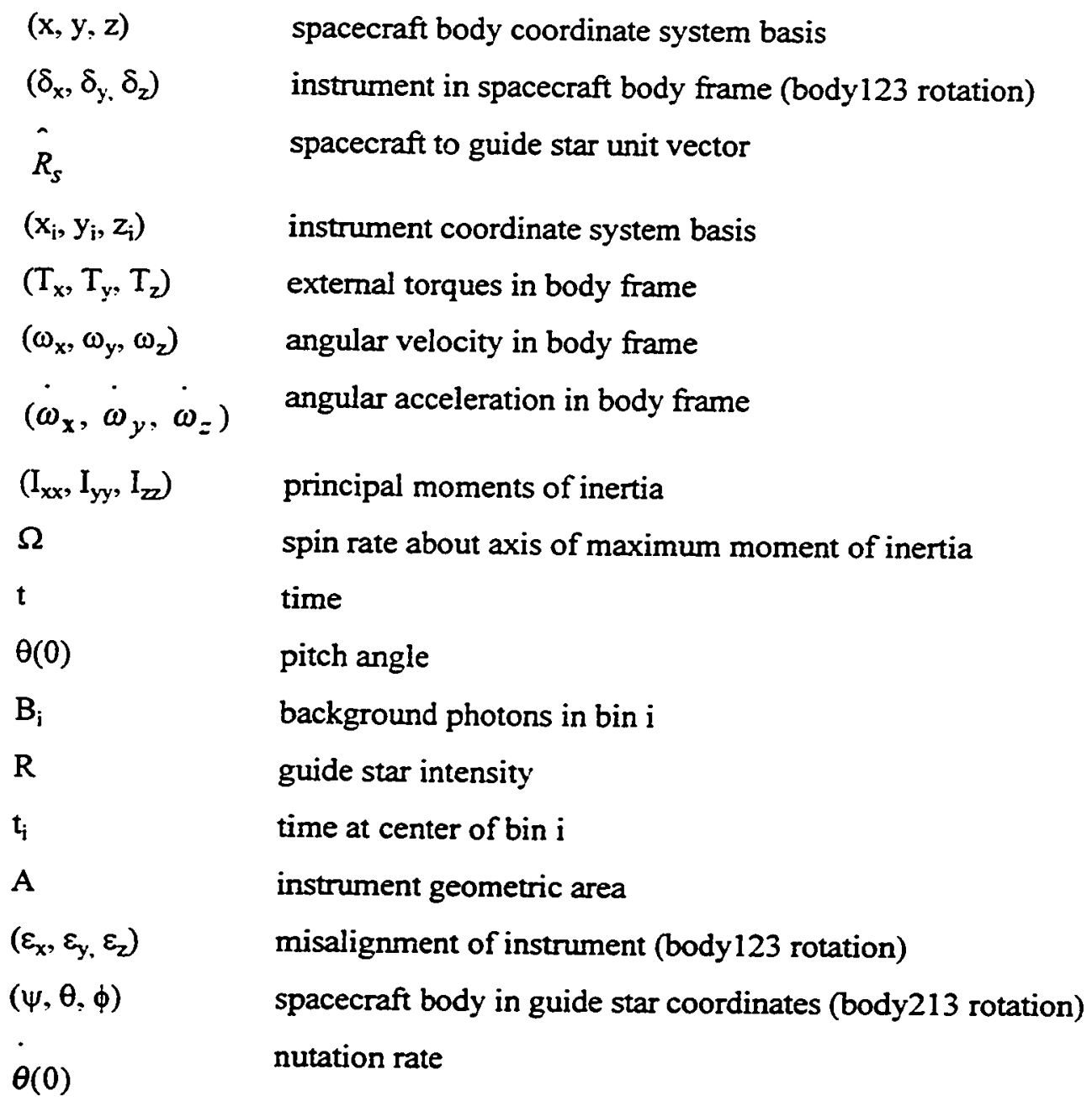




\begin{tabular}{|c|c|}
\hline \multicolumn{2}{|c|}{ HEAO Data and Model Fitting } \\
\hline $\mathrm{C}_{\mathbf{j r}}$ & raw counts from module $\mathrm{j}$ \\
\hline$C_{j}$ & counts from module $\mathrm{j}$ corrected for instrument dead time \\
\hline$\tau_{\mathrm{d}}$ & dead time \\
\hline$\tau_{b}$ & bin size \\
\hline$Y_{j i}$ & model counts in bin $\mathrm{i}$, module $\mathrm{j}$ \\
\hline $\mathrm{B}_{\mathrm{ji}}$ & model background counts in bin $i$, module $j$ \\
\hline $\mathrm{T}_{\mathrm{ji}}$ & collimator transmission in bin $\mathrm{i}$, module $\mathrm{j}$ \\
\hline $\mathrm{B}_{\mathrm{j} 0}, \mathrm{~B}_{\mathrm{jf}}$ & linear background fit, module $\mathrm{j}$ \\
\hline$B_{j 0}, B_{j f}$ & linear background fit, module $\mathrm{j}$ \\
\hline $\mathrm{B}_{\mathrm{ji}}$ & model background counts in bin $i$, module $j$ \\
\hline $\mathrm{R}$ & model guide star intensity \\
\hline $\mathrm{N}$ & number of bins in scan \\
\hline $\mathrm{i}$ & bin number \\
\hline$T_{\alpha \mathbf{i}}$ & transmission in pitch at bin $\mathrm{i}$ \\
\hline$T_{p i}$ & transmission in roll at bin $\mathrm{i}$ \\
\hline$t_{i}$ & time at center of bin $i$ \\
\hline $\mathrm{i}_{\mathrm{p}}$ & model bin number where roll angle is zero \\
\hline$\mu$ & total intensity \\
\hline$\delta_{\mathrm{jx}}$ & collimator pitch offset, module $j$ \\
\hline$\delta_{\mathrm{jz}}$ & collimator roll offset, module $j$ \\
\hline$\varepsilon_{\mathrm{y}}$ & collimator yaw misalignment \\
\hline \multicolumn{2}{|c|}{$X$-ray Time Keeping } \\
\hline$u_{0}$ & raw pulsar signal \\
\hline$\omega_{1}$ & prefiltered pulsar fundamental frequency \\
\hline $\mathrm{u}_{\mathrm{l}}$ & prefiltered pulsar signal \\
\hline$u_{d}$ & phase detector signal \\
\hline$\omega_{2}$ & VCO output frequency \\
\hline
\end{tabular}




\begin{tabular}{|c|c|}
\hline $\mathrm{u}_{2}$ & VCO output signal \\
\hline$\omega_{0}$ & VCO center frequency \\
\hline$u_{\mathrm{r}}$ & loop filter output signal \\
\hline$\theta_{e}$ & PLL phase error \\
\hline$\theta_{1}$ & preniltered pulsar phase \\
\hline$\theta_{2}$ & VCO output phase \\
\hline$F_{1 f}(s)$ & loop filter transfer function \\
\hline $\mathrm{K}_{\mathrm{f}}$ & loop filter gain \\
\hline$\tau_{1}, \tau_{2}$ & loop filter pole/zero locations \\
\hline $\mathbf{s}$ & LaPlace operator \\
\hline$\omega_{\mathrm{vco}}$ & VCO center frequency \\
\hline$\Theta_{2}$ & VCO output phase, LaPlace domain \\
\hline $\mathrm{U}_{\mathrm{f}}(\mathrm{s})$ & loop filter output signal, LaPlace domain \\
\hline $\mathrm{U}_{0}(\mathrm{~s})$ & pulsar raw signal, LaPlace domain \\
\hline $\mathrm{U}_{1}(\mathrm{~s})$ & pulsar prefiltered signal, LaPlace domain \\
\hline $\mathrm{U}_{\mathrm{d}}(\mathrm{s})$ & phase detector output signal, LaPlace domain \\
\hline$F_{p r}(s)$ & prefilter transfer function \\
\hline $\mathrm{H}(\mathbf{s})$ & PLL linear transfer function \\
\hline$\omega_{\mathrm{n}}$ & PLL natural frequency \\
\hline$\zeta$ & PLL damping ratio \\
\hline$\Delta \omega_{\mathrm{H}}$ & hold range \\
\hline$\Delta \omega_{\mathrm{P}}$ & pull-in range \\
\hline$\Delta \omega_{\mathrm{L}}$ & lock-in range \\
\hline$T_{L}$ & lock time \\
\hline$\Delta \omega_{\mathrm{PO}}$ & pull-out range \\
\hline$\Delta \mathrm{t}$ & time error \\
\hline$\Delta \mathrm{x}$ & position error \\
\hline c & speed of light \\
\hline$\Delta f$ & frequency offset \\
\hline
\end{tabular}




$\begin{array}{ll}v_{\text {los }} & \text { instrument velocity in the direction of the line of sight to the pulsar } \\ \mathrm{f}_{\text {pulsar }} & \text { pulsar fundamental frequency } \\ \mathrm{C}_{\mathrm{i}} & \text { total photons in bin } \mathrm{i} \\ \mathrm{B}_{\mathrm{i}} & \text { photons in bin } \mathrm{i} \text { due to background } \\ \mathrm{N}_{\mathrm{i}} & \text { photons in bin } \mathrm{i} \text { due to Poisson noise } \\ \mathrm{S}_{\mathrm{i}} & \text { photons in bin } \mathrm{i} \text { due to steady part of pulsar } \\ \mathrm{P}_{\mathrm{i}} & \text { photons in bin i due to pulsar oscillations } \\ \mathrm{a}_{1} \ldots \mathrm{a}_{4}, \mathrm{~b}_{1} \ldots \mathrm{b}_{4} & \text { Crab pulsar fit parameters } \\ \mathrm{i} & \text { bin number } \\ \mathrm{P}_{1 \mathrm{i}} & \text { mean rate in bin } \mathrm{i} \text { from first pulse } \\ \mathrm{P}_{2 \mathrm{i}} & \text { mean rate in bin i from second pulse } \\ \eta_{c} & \text { normalizing parameter } \\ \sigma_{\mathrm{i}} & \text { standard deviation of noise in bin } \mathrm{i} \\ \mathrm{K}_{\mathrm{vco}} & \text { VCO gain } \\ \mathrm{K}_{\text {loop }} & \text { PLL loop gain } \\ \mathrm{K}_{\mathrm{pd}} & \text { phase detector gain } \\ \mathrm{h}_{\mathrm{inp}} & \text { amplitude of prefiltered pulsar fundamental }\end{array}$




\section{Chapter 1: Introduction}

\subsection{Problem Statement}

Since the discovery in 1962 that many stars emit x-rays, the scientific community has invested a great deal of time and effort in characterizing and understanding the $x$-ray sky. This knowledge has reached a level of maturity that will now allow $\mathrm{x}$-ray sources to be used as a set of inertial references to measure the attitude, time and position of a satellite. First proposed by Dr. Kent Wood in a 1993 paper (Wood, 93), the process of using $\mathrm{x}$-ray sources as references for measuring the state of a spacecraft is referred to as "x-ray navigation."

Currently available instrumentation makes it possible to determine the attitude, time and position of a satellite in an autonomous or semi-autonomous fashion. In fact, technology such as GPS may soon make it possible, in some instances, to measure these parameters with a single, integrated instrument. $X$-ray navigation is another tool that will ultimately make it possible to determine the state of a spacecraft with a single, integrated instrument. Moreover, x-ray navigation can be used on satellites on interplanetary missions as well as those in low earth orbit. This new concept will also make it possible 
to use the payloads of satellites dedicated to $x$-ray science to track the satellite time, attitude and position thus obviating the need for instruments specifically dedicated to measuring these parameters. In this thesis, the feasibility of $x$-ray navigation will be explored. This feasibility is demonstrated through a combination of simulation and experimentation.

The launch of the Unconventional Stellar Aspect Experiment (USA) aboard the Advanced Research and Global Observation Satellite (ARGOS) in late 1996 will be the first step in developing this new class of sensors. Simulations of $\mathrm{x}$-ray timing concepts were performed with this platform in mind. Furthermore, extensive design, analysis and manufacturing work on USA was done to support the experiment. This design work serves as an excellent case study in the design of satellite systems and will be described in detail.

The High Energy Astronomical Observer (HEAO-1) was a satellite launched in 1977 and dedicated to making observations of the sky in the $x$-ray regime. Data from this mission is available from a database at SLAC and was used to demonstrate the feasibility of attitude determination of a spinning spacecraft from observations of $x$-ray sources.

\subsection{History of X-ray Missions}

The growth in the field of $x$-ray astronomy coincides directly with the development of launch vehicles capable of placing instruments sufficiently above the atmosphere of the earth to detect $x$-rays (Table 1.1). This is due to the fact that the atmosphere is opaque to light in the $x$-ray regime $(\sim 0.1 \mathrm{keV}$ to $\sim 1 \mathrm{MeV})$. The first detection of $\mathrm{x}$-rays from a nonterrestrial source came in 1949 when Geiger counters flown on a sounding rocket showed that the sun emits x-rays (Bradt et al 1992). In 1962 another sounding rocket experiment detected $x$-rays from Sco-X1, the first indication that $\mathrm{x}$-rays are emitted by bodies outside the solar system (Ibid). Early experiments were also performed using high altitude balloons to carry larger detectors to lower altitudes than the sounding rockets, but for much longer periods of time. These experiments typically 
looked for the higher energy photons that could penetrate the remaining atmosphere above the balloons.

The first satellite to make observations of the $\mathrm{x}$-ray sky was the Orbiting Solar Observatory 3 (OSO-3) launched in 1967. Among other things, it produced an all-sky map of the diffuse $x$-ray background in the $10-40 \mathrm{keV}$ range (Ibid). The Vela series of satellites launched by the Department of Defense also detected celestial $x$-ray sources in this time period.

With its launch in 1970, Uhuru became the first satellite to be dedicated entirely to celestial X-ray astronomy (Ibid). Uhuru was a spinning satellite with a spin period of about 12 minutes that was placed into a nearly equatorial orbit. Using a set of proportional counters sensitive to $2-20 \mathrm{keV}$ x-rays fitted with $1^{\circ} \times 10^{\circ}$ collimators (full width half maximum), Uhuru produced the first detailed $x$-ray catalog, a catalog that contains 339 objects with intensities as low as about $10^{-3}$ of the intensity of the Crab nebula (Ibid). This map showed the clustering of sources in the galactic plane as well as a large number of extragalactic sources spread isotropically in the sky (Ibid). Towards the end of its mission, Uhuru would be the first satellite to use $x$-ray sources as an attitude reference.

Launched in 1972, Copernicus (OAO-3) was the first mission to provide detailed ( 10 arc-minute) resolution of an extended x-ray source. The Skylab missions of 19731974 used two grazing incidence telescopes to take an image of the sun in x-rays with 2 arc-second resolution (Ibid). Using a coded aperture mask to image the sun in $\mathrm{x}$-rays was first proposed by Dicke in 1961 (Dicke, 1968). This foreshadowed wide-field pin-hole cameras flown on Ariel-5 in 1976 and the first use of a coded mask to image the galactic center in 1978 (Bradt et al, 1992). The Ariel-5 mission also found several $x$-ray pulsars with periods of minutes.

Between 1977 and 1981 NASA operated two large scientific satellites dedicated to $x$-ray astronomy. These were the High Energy Astronomy Observatories, HEAO-1 and HEAO-2 (Einstein). HEAO-1 was a spinning survey mission while HEAO-2 was a pointed imaging mission. The third satellite in the series, HEAO-3, was a cosmic ray and gamma-ray mission. 


\begin{tabular}{|l|l|l|l|}
\hline \multicolumn{1}{|c|}{ Mission } & \multicolumn{1}{|c|}{ Lifetime } & \multicolumn{1}{c|}{ Mission Class } & \multicolumn{1}{c|}{ Science } \\
\hline OSO-3 & $1967-1968$ & Spinning & Diffuse Background \\
\hline OSO-5 & $1969-1972$ & Spinning & Diffuse Background \\
\hline Vela Series & $1969-1979$ & & X-ray Bursts \\
\hline Uhuru & $1970-1973$ & Spinning & Catalog \\
\hline OSO-7 & $1971-1973$ & Spinning & Catalog \\
\hline Copernicus & $1972-1981$ & Pointed & SNR Mapping \\
\hline ANS & $1974-1976$ & Pointed & X-ray Bursts \\
\hline Salyut-4 & $1974-1975$ & Pointed & Cyg X-1, Her X-1 \\
\hline Ariel-5 & $1974-1980$ & Spinning & Catalog \\
\hline Apollo-Soyuz & 1975 & Pointed & SMC X-1 Pulsations \\
\hline SAS-3 & $1975-1979$ & Spinning/Pointed & Precise Positions \\
\hline OSO-8 & $1975-1978$ & Spinning & \\
\hline HEAO-1 & $1977-1979$ & Spinning/Pointed & All-sky Catalog \\
\hline Einstein & $1978-1981$ & Pointed, Imaging & Imaging \\
\hline Ariel-6 & $1979-1981$ & & GX 1+4 Position \\
\hline Hakucho & $1979-1984$ & Spinning & X-ray Bursts \\
\hline Astron & $1983-1988$ & Pointed & Her X-1 Low State \\
\hline Tenma & $1983-1984$ & Spinning & \\
\hline EXOSAT & $1983-1986$ & Pointed & QPO's \\
\hline Ginga & $1987-1991$ & Pointed & AGN's \\
\hline Kvant/Mir & $1987-$ & & \\
\hline Granat & $1989-$ & Pointed & Catalog \\
\hline ROSAT & $1990-$ & Pointed & Spectra \\
\hline Astro-1 & 1990 & & \\
\hline Spartan & & Gainted & \\
\hline
\end{tabular}

Table 1.1: X-ray Missions (Bradt et al, 1992) 
The HEAO-1 mission was a spinning platform consisting of four primary experiments (Ibid):

1. Large Area Sky Survey (LASS or HEAO-A1): Four proportional chambers totaling $1.0 \mathrm{~m}^{2}$ that were sensitive to $\mathrm{x}$-rays in the $1-20 \mathrm{keV}$ range. Each detector had a collimator and was designed to use the spinning action of the satellite to survey the entire x-ray sky. The HEAOAl experiment produced a catalog of sources with intensities as low as $10^{-3}$ the intensity of the Crab that included $842 \mathrm{x}$-ray sources. The raw data from these surveys was used in the attitude determination experiments described later. The HEAO-Al data also had excellent time resolution which may allow for future experiments in $x$-ray time determination studies.

2. Cosmic X-ray Experiment (CXE): An array of proportional chambers $0.4 \mathrm{~m}^{2}$ in area designed to study the diffuse $\mathrm{x}$-ray background in the 0.2 $\mathrm{keV}$ to $60 \mathrm{keV}$ range.

3. Modulation Collimator (MC): This experiment was designed to determine the position of $x$-ray sources to about one arc-minute accuracy. 4. A high energy experiment for observations up to $\sim 10 \mathrm{MeV}$.

The Einstein spacecraft was a powerful $x$-ray imaging mission that empioyed grazing-incidence focusing optics for the first time in celestial $x$-ray astronomy. This system was sensitive to energy in the range of $0.1-4 \mathrm{keV}$ and could resolve sources as dim as $10^{-7}$ the intensity of the Crab (Ibid).

The 1980's saw several satellites that extended the knowledge of the $\mathrm{x}$-ray sky in a variety of ways. These included EXOSAT, Hakucho, Tenma and Ginga (Ibid). 
These missions of the past three decades have led to a body of knowledge in x-ray astronomy that will enable the $x$-ray navigation concept. Some of the important characteristics of the $x$-ray sky are summarized below:

1. There are a relatively small number of bright sources in the $x$-ray sky. The 842 sources in the HEAO all-sky catalog vary by 4 orders of magnitude in intensity from brightest to dimmest.

2. Many sources reside in the galactic plane, but there are significant sources in other areas of the sky.

3. There is a faint diffuse $x$-ray background whose origin is not well defined at this time.

4. The sources and background tend to be fairly faint and observations of them may be modeled as Poisson processes.

5. Many of the sources have been identified as $x$-ray pulsars and have periods that range from tens of milliseconds to months.

\subsection{Satellite Navigation Principles}

\subsubsection{Attitude Determination}

The sensors designed to measure the attitude of a spacecraft can be grouped according to their reference objects. Sun-sensors measure the pointing angles of the spacecraft relative to the sun, horizon-sensors use the limb of the earth as a reference, and star trackers use optical or ultraviolet measurements of the locations of guide stars. Recently, it has been shown that the GPS system can be used to determine the attitude of LEO satellites by measuring the relative distances to the individual GPS satellites (Cohen, 1991). With the exception of GPS, all of these sensors are sensitive to emissions from celestial bodies in the infra-red, optical, or ultraviolet wavelengths.

Many of the celestial objects visible in optical wavelengths have significant radiant signatures in other wavelengths. In particular recent studies of the sky in $\mathrm{x}$-ray 
wavelengths indicate that a sensor designed to detect such objects would make an excellent star tracker. In fact, the Uhuru satellite was the first satellite to act as an x-ray star tracker. In December of 1972 the optical star trackers used to provide one arc-minute level orientation measurements failed. In order to superpose the count rates from successive scans the aspect data from the magnetometers and sun sensor were used. This was supplemented by using the locations of bright $\mathrm{x}$-ray sources to synchronize the scans (Forman et al 1978).

Dr. Wood proposed two basic designs of an x-ray star tracker (Wood, 1993). The first employs a coded aperture mask and a pixelated detector to image the sky directly. The image is then correlated with a map of the $x$-ray sky using any of the methods developed for more traditional star trackers. The second method would use a collimated detector (non-pixelated) and a mechanical scanning device to scan over a known source repeatedly. This is similar to traditional star scanners that search the sky either mechanically or electronically to keep a guide star in the field of view. If a collimated $x-$ ray detector is placed on a spinning spacecraft, the scanning mechanism is supplied by the spacecraft and a separate scanning mechanism is not necessary.

There are several advantages to measuring attitude by reference to $x$-ray emitting objects:

1. The sparsity of the $\mathrm{x}$-ray sky simplifies the pattern recognition problem.

2. The periodicity associated with some sources could be an excellent way of identifying guide stars and thereby reducing the scope of the pattern recognition problem.

3. X-ray detectors are robust and do not require any optics or special cooling.

4. The measurement of attitude is done in an inertial frame and is, in principle, capable of accuracies comparable to conventional star trackers.

5. The measurement of time, attitude and position can be done autonomously by a single integrated instrument. 


\subsubsection{Time Keeping}

Time on spacecraft has typically been kept by a local crystal oscillator with periodic corrections from the ground. A quartz crystal oscillator can be made stable to a part in $10^{6}$ without much effort. The Transit Navigation Satellites had a requirement for a very stable frequency source over short periods of time. These used a double dewar system to isolate an oscillator thermally, thus achieving a local frequency stability of a part in $10^{9}$ (Black, 1990).

The Gravity Probe A (GP-A) experiments flow by Bob Vessot used hydrogen masers to get a time reference that was stable to a part in $10^{14}$ over integration periods from one minute to one hour. Launched on a sounding rocket, the payload clock was compared to the time kept by a ground based hydrogen maser as a test of the Theory of Relativity (Vessot, 1995).

While these examples show that it is possible to fly extremely stable local clocks on satellites, they also show that it is very difficult and very costly to do so. Future missions that require accurate local timing will have to use new methods. With the advent of GPS, spacecraft in low earth orbit can now keep accurate local time semiautonomously (Hoech, et al, 1994).

A more flexible and potentially more accurate method of keeping accurate local time on satellites in any orbit would use the many $x$-ray emitting objects that are also $x$ ray pulsars. These pulsars have been shown to have periods with stability equivalent to atomic clocks and can be used as a completely autonomous absolute time reference (Wood, 93). By observing an x-ray pulsar over an extended period of time, an inexpensive local crystal oscillator can be corrected for long term drift by using a phaselocked loop to lock it in frequency and phase to the pulsar. By extension, several local oscillators, each with a different center frequency, can be locked to several different pulsars simultaneously. This will provide an autonomous measure of time from scales of milliseconds to months allowing for autonomous initialization as well as a redundancy in the time measurement. This technology will enable missions to be flown that require accurate time information to be delivered without ground intervention. 


\subsubsection{Position Determination}

The position of a satellite in orbit has traditionally been determined by ground based tracking stations. After estimating the ephemeris of the orbit, these parameters are uplinked so that the satellite can estimate its position as a function of time. Methods of determining the position of a spacecraft autonomously have been suggested, but have yet to be implemented on operational satellites. A great deal of research was performed in the late 1960's and early 1970's to perfect some of these techniques. These included tracking known landmarks on the earth, but this method was hindered at times when cloud cover obscured landmarks (White, 1975). The TRIAD-1 satellite used its drag-free reference to improve the accuracy of ephemeris predictions by removing the uncertainty in the propagation of the orbit parameters caused by unmodelled and random disturbances. By using the drag free system to cancel these disturbances, the satellite position could be projected 15 days into the future with an along track navigation error of $100 \mathrm{~m}$ and a slant range navigation error of $100 \mathrm{~m}$ (Staff of the Space Department, 1974).

Other methods involved measuring the time of occultation of a guide star by the earth or the moon. One interesting proposal would measure the atmospheric refraction from a known star as that star sets behind the earth (White et al, 1985). Recent studies

suggest that such an approach can pinpoint the position of a satellite to hundreds of meters.

With the GPS system coming on line, it will be possible to determine the position of a satellite in low earth orbit semi-autonomously. That is, the satellite will not need any direct interference from ground controllers, but will be dependent on the GPS system for its position updates. The GPS system is, in turn, dependent on ground control for its proper operation.

The position of a satellite in orbit can be determined by observing the occultation of an X-ray guide star by the earth or the moon (Wood, 1993). By measuring the occultation of several sources in different directions, a position fix can be made 
autonomously. This would require some sort of $x$-ray imaging system (coded mask, grazing incidence mirrors or scanning collimator).

\subsection{USA Experiment Design Issues}

The Stanford Linear Accelerator Center (SLAC) and the Naval Research Laboratory (NRL) are cooperating on the design, manufacturing and operation of the Unconventional Stellar Aspect Experiment (USA). USA is an x-ray telescope schedule to be launched into low earth orbit aboard ARGOS, a satellite funded as part of the Air Force STEP Program and built by Rockwell International for launch in early 1997. This experiment involves mounting two large $x$-ray detectors in a gimbaled structure. SLAC was responsible for the design, manufacturing and test of the support structure as well as the thermal design of the entire experiment. Additionally, SLAC was responsible for the design, manufacturing and testing of three collimators (two flight, one spare) used in the experiment as well as various flight and ground software issues. In order to build this experiment on the short timescale and limited budget it was afforded, it was necessary to work closely with other team members at the Naval Research Laboratory, Rockwell, the Air Force, and the Aerospace Corporation. This work offers an excellent case study for designing satellite systems in small semi-autonomous groups.

\subsection{USA/HEAO Navigation Experiments}

The Unconventional Stellar Aspect Experiment will provide a unique platform for the demonstration of $x$-ray navigation (Wood et al USA). Two RS-6000 class (20 Mips) computers will have real-time access to the data from the two USA detectors. This data can then be used to determine the state of the spacecraft. A truth measurement of time and position will be available from a GPS receiver on board the spacecraft while the attitude of the spacecraft will be available from the ADACS system. The time and 
attitude truth measurements will be available in real time while the position measurement will be available in post-processing.

USA will be used as an experimental scanning collimator system, thus demonstrating an operational x-ray attitude determination system. By scanning over a small section of the sky where a guide star is known to reside, an "image" of that section of the sky can be formed. The location of the guide star within this image is then used to determine the attitude of the satellite in real time.

USA can also be used to track the local time using x-ray pulsars. A simple timing experiment would involve programming the RS-6000 computer to run as a digital phaselocked loop with the stream of scientific $x$-ray data as its input. The performance of this loop can then be compared to the truth measurement and a simple performance measure sent down in the data stream.

USA will also make measurements of the position of the ARGOS satellite by observing the occultation of known guide stars by the earth and the moon.

Fortunately, the data from previous $\mathrm{x}$-ray missions can be used to prove some of these navigation concepts now. As previously discussed, the High Energy Astronomical Observatory is a satellite that flew in the late 1970's and mapped the sky in the $x$-ray regime. The data from this experiment are still available and have been used to demonstrate the feasibility of $x$-ray attitude determination on spinning spacecraft.

\subsection{Contributions}

This thesis is dedicated entirely to exploring the possibilities of $\mathrm{x}$-ray navigation. To this end a great deal of effort was expended to test the ideas developed here through simulation or experimentation. Furthermore, the USA Experiment is seen as a critical tool for exploring these concepts in the future. The main contributions of this thesis are as follows:

1. Designed, manufactured and tested the structural components of the Unconventional Stellar Aspect Experiment. This demonstrated that dip brazing can be 
used as a cost efficient manufacturing method for large space structures whose design is driven by stiffness considerations and not strength considerations.

2. Explored the use of $x$-ray imaging devices (star mapper) on inertially pointed and/or slowly rotating vehicles as an attitude measurement instrument, showing that measurement update rates in the $100 \mathrm{~Hz}$ range are feasible.

3. Explored the use of simple collimated $x$-ray detectors on spinning spacecraft as an attitude measurement instrument (star scanner). A theoretical framework for the design of both single detector and differential detector instruments was provided including a discussion of error sources. Errors due to the polhode motion of a spinning body and manufacturing misalignments of the instrument were described and models for their estimation were suggested.

4. Demonstrated the operation of single detector and differential detector star scanners using data from the High Energy Astronomical Observatory, a spinning satellite launched in 1978. Four different detector schemes were demonstrated: single detector, differential detector, single detector with a second order model and differential detector with a second order model.

5. Explored the use of an $x$-ray pulsar as a stable, autonomous frequency reference by simulating the performance of a phase locked loop in acquiring and locking onto the Crab Nebula pulsar. A discussion of potential error sources present in such an application was included.

\subsection{Thesis Outline}

When I first joined the SLAC/NRL team, it was with the understanding that I would be responsible for the structural and thermal analysis necessary to verify the design of the Unconventional Stellar Aspect Experiment. That responsibility soon grew to include the design, manufacturing and testing of the USA gimbal structure. This required a close working relationship with both those physicists building the experiment and those who would be the end-users of the data generated by USA. This relationship led to my appreciation for the possibilities of $\mathrm{x}$-ray navigation - the subject of this work. For this 
reason, I have arranged this thesis in two sections, starting with the design of the Unconventional Stellar Aspect Experiment and then moving on to discuss methods for using the $x$-ray sky to measure the orientation of a spacecraft and to provide it with a stable frequency reference.

Chapter 2 covers the design, manufacturing and testing of the Unconventional Stellar Aspect Experiment. This begins with a general description of the mission and experiment and the design requirements. An integrated design and analysis process is discussed in which a finite element model of various structural design options was used. This lead to a novel design for such a large structure as USA that was made possible by using a process called dip brazing. A complete discussion of the dip brazing process and the USA Structural Test Program is included. Finally, Chapter 2 ends with a summary of the USA thermal analysis, including a description of the thermal design and the various operational modes that are important considerations in a thermal design.

The concept of x-ray attitude determination is introduced in Chapter 3. First. a feasibility study that suggests $x$-ray imagers can be used as precision pointing devices (star mappers) on inertially pointed or slowly spinning spacecraft is presented. The second part of Chapter 3 develops the idea of using collimated $x$-ray detectors on a spinning spacecraft to determine its orientation (star scanners). This begins with a description of the signal generated by such an instrument given the general rotational motion of the spacecraft. Two models for the operation of $\mathrm{x}$-ray star scanners are presented - a first order model that assumes the spin axis is properly aligned with the instrument axes and a second order model that includes misalignment effects. Three general instruments are described - a single detector/collimator system, a differential detector/collimator system using two detectors and a differential detector/collimator system using a single detector and two collimators.

In Chapter 4 the concept of $x$-ray attitude determination is demonstrated by using archival data from the HEAO-1 mission. Four different cases are described and their results presented:

1. Single detector, single collimator with first order model

2. Dual detector, dual collimator differential instrument with first order model 
3. Single detector, single collimator with second order model

4. Dual detector, dual collimator differential instrument with second order model The concept of autonomous $\mathrm{x}$-ray timing is discussed in Chapter 5. A simulation of a phase locked loop used to lock onto the Crab Nebula pulsar is described. This includes a general discussion of phase locked loops as well as some of the errors to be anticipated in using an $\mathrm{x}$-ray pulsar as an autonomous frequency reference.

Chapter 6 provides an overview of the thesis and an extensive list of suggestions for future research. 


\section{Chapter 2: Design Aspects of the Unconventional Stellar Aspect Experiment}

\subsection{USA Mission}

The Unconventional Stellar Aspect Experiment (USA) will provide a unique platform for experiments in the area of x-ray navigation. Concurrently, USA is tasked to provide large quantities of high resolution $x$-ray timing data on 30 of the most $x$-ray luminous stellar objects known. Observations for each object will be made with timing resolution on the order of $10^{-6} \mathrm{sec}(1 \mu \mathrm{sec})$ for about one month of total elapsed time. As previously described, USA is an experiment that will be flown as one of several on an Air Force satellite called the Advanced Research and Global Observation Satellite (ARGOS). USA will be mounted on the aft or antivelocity face of ARGOS (Figure 2.1). From this position USA will be able to slew its x-ray detectors to track any target in its field of regard continuously for about 20 minutes per orbit. ARGOS is in a sun-synchronous orbit at an altitude of $450 \mathrm{n}$.mi. (Figure 2.2, Table 2.1). This will allow USA to observe 


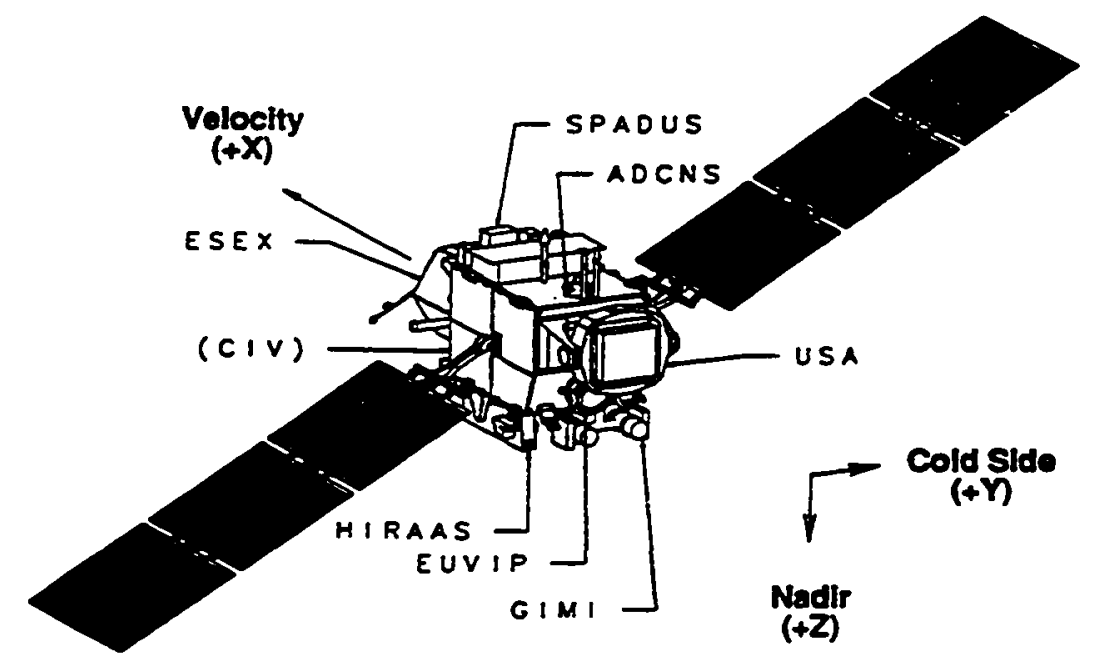

Figure 2.1: Advanced Research and Global Observation Satellite

Notes:

1. Orbit in $X Y$ Plane

2. Sun Line in $X Z$ Plane

3. $(X, Y, Z)$ ore ECl Coordinates

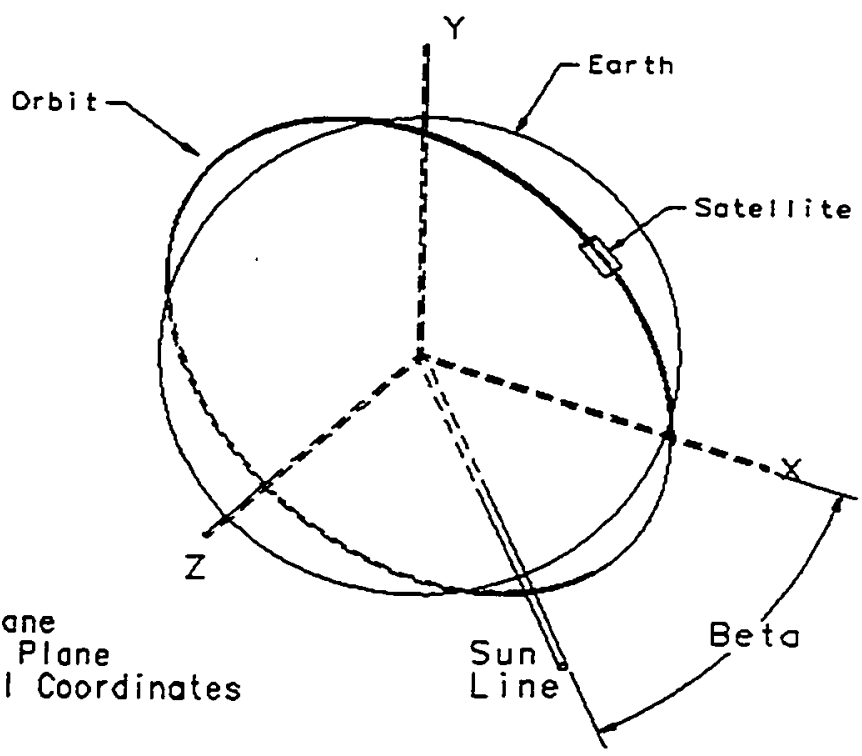

Figure 2.2: Advanced Research and Global Observation Satellite Orbit 
the entire celestial sphere over its lifetime as the precession of the orbit brings targets into the instrument field of regard.

\begin{tabular}{|l|l|}
\hline Orbit Apogee & $450+/-10 \mathrm{n} . \mathrm{mi}$. \\
\hline Orbit Perigee & $450+/-10 \mathrm{n} . \mathrm{mi}$. \\
\hline Orbit Inclination & $98.7+/-0.06$ degrees \\
\hline Beta Angle (BOL) & $36+/-3$ degrees \\
\hline Beta Angle (EOL Min) & 15 degrees \\
\hline Beta Angle (EOL Max) & 54 degrees \\
\hline Orbit Period & 101.6 min (nominal) \\
\hline
\end{tabular}

Table 2.1: USA Orbit Parameters

\subsection{USA General Description}

The Unconventional Stellar Aspect Experiment (Figure 2.3) consists of two proportional chambers in a two-axis gimbal system. This gimbal system allows for full movement of the detectors in pitch and yaw so that any source in the field of regard can be tracked during an orbit $\left(+70^{\circ} /-110^{\circ}\right.$ pitch, $+/-90^{\circ}$ yaw). The proportional chambers were refurbished after a previous mission on the shuttle called Spartan. Combined they weigh approximately $210 \mathrm{lb}$. This includes the two 12 in spherical gas tanks carried on the backs of the detectors. A complete description of the mass properties of the USA experiment is given in Table 2.2. Each proportional chamber consists of the following components (see Figure 2.4):

1. One aluminum proportional chamber made from the detector body and detector backplane. The detector backplane is bolted to the body with a viton o-ring between them to provide a vacuum tight seal. The detector electronics are located on the outside of the detector backplane. The proportional chamber is filled with P-10 gas ( $90 \%$ Argon, 


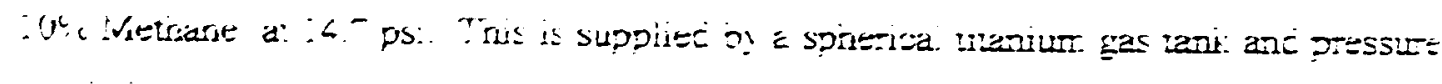
reguiatior sysierr.

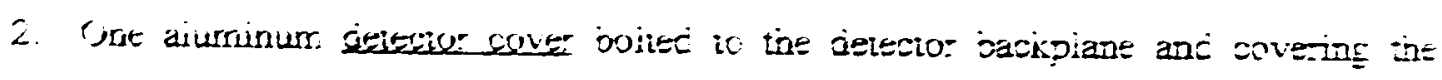

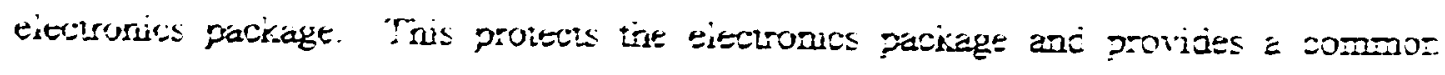
radiator for al: of the tiectronits.

3. Tre 12 ir diarreter sphtricai tivanium yas borie containing ?-10 gas a: 2700 ps. This is bolted u the detecior backplane. The gas botile contains no iniena: jea: generavo=

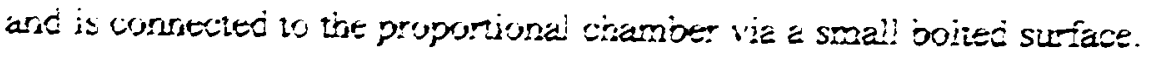

4. (Nit 2.5 micron thich mviar windou heid between the deiecior body and the collirratur trame. This wincuw provides containmen for the P-10 gas wivile aosorbing a mirimum of the incuming $x$-rays. This is pazicularly imporant for $x$-rays with energies of about $1 \mathrm{keV}$ since the presence of the mylar uindou defines the lower ead of the detector energy acceptance.

5. (Jne aluminum collinator frame holding two collimator modules against the mylawindow. The frame is bolted against the detector body with another viton o-ring between the two, making a seal that prevents the loss of chamber gas.

6. I wo sollimator modules made from copper hex-cell. The modules are held within the collimatur frame by an interference fit and provide support to the mylar window. A fine wire mesh is placed between the window and the collimator modules for additional support and to protect the window from any small burs on the collimators.

7. One heal shield and aluminum heal shield frame. The heat shield is made from 2.5 micron thick second surface aluminized mylar and is suspended 0.25 in above the collimator modules by the heat shield frame. This keeps the sun heat input through the lace of the collimator to a minimum. 


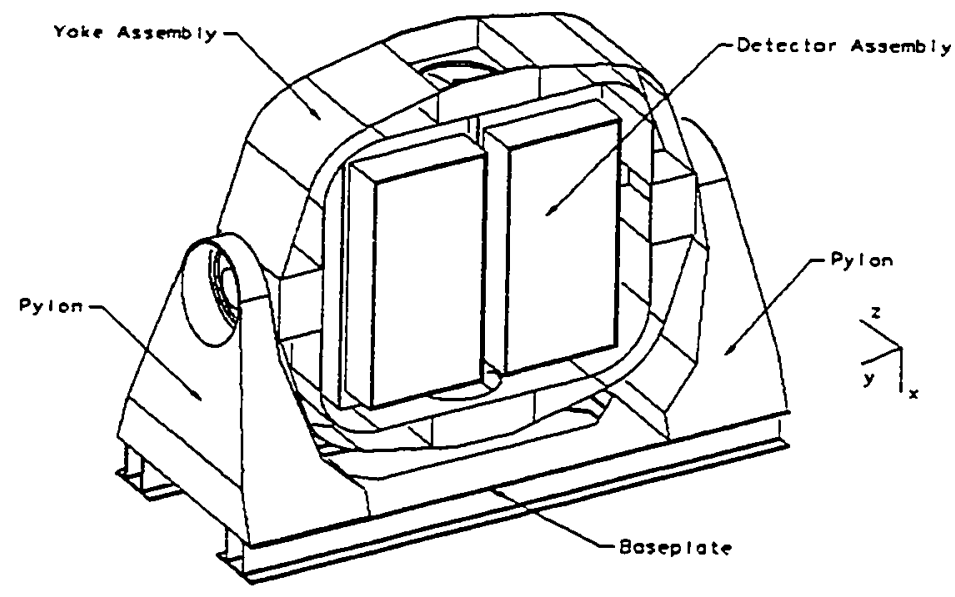

Figure 2.3: Unconventional Stellar Aspect Experiment

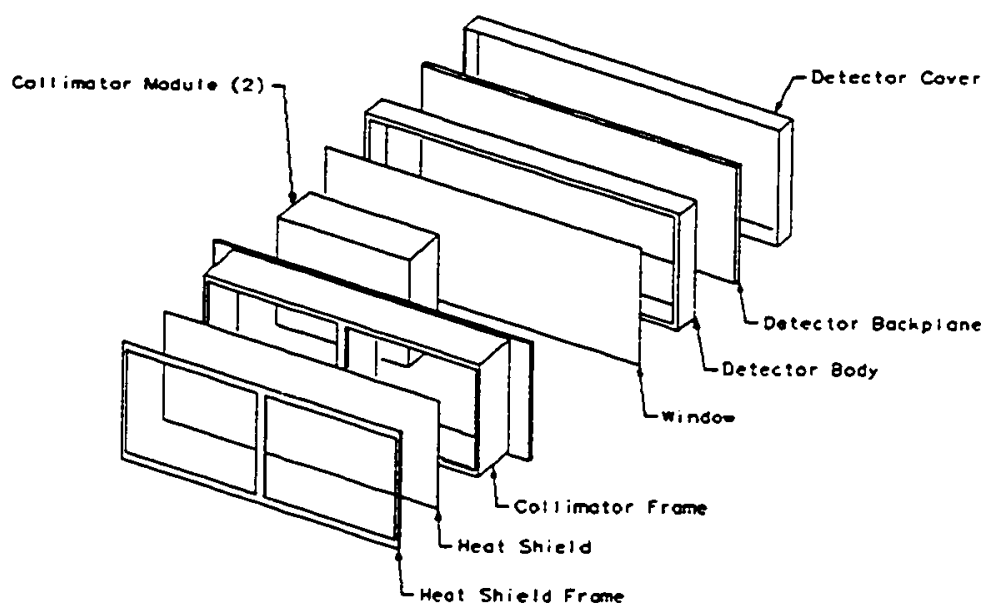

Figure 2.4: Unconventional Stellar Aspect Experiment Proportional Chamber 


\begin{tabular}{|l|l|c|l|}
\hline \multicolumn{1}{|c|}{ Component } & \multicolumn{1}{|c|}{ System } & Quantity & \multicolumn{1}{c|}{ Mass (each) (lb) } \\
\hline Collimator & Detector & 2 & 15.1 \\
\hline Collimator Frame & Detector & 2 & 10.3 \\
\hline Heat Shield Frame & Detector & 2 & 10 \\
\hline $\begin{array}{l}\text { Proportional } \\
\text { Chamber }\end{array}$ & Detector & 2 & \\
\hline $\begin{array}{l}\text { Detector Backplane } \\
\text { \& Electronics }\end{array}$ & Detector & 2 & 39.8 \\
\hline Gas Tank & Detector & 1 & 24.1 \\
\hline Detector Subtotal & Detector & 2 & 210.6 \\
\hline Pylon & Gimbal & 2 & 22 \\
\hline Motor & Gimbal & 2 & 22 \\
\hline Encoder & Gimbal & 1 & 51 \\
\hline Baseplate & Gimbal & 1 & 20 \\
\hline Central Electronics & Gimbal & Yoke & 64 \\
\hline Yoke & Yoke/Gimbal & 1 & 43 \\
\hline Wire Harnesses & USA & 2 & 5.6 \\
\hline USA Total & Tabl 2: USA & 2 & 2 \\
\hline
\end{tabular}

Table 2.2: USA Mass Properties

The two detectors are mounted in an aluminum frame which is suspended inside of an aluminum yoke assembly by an encoder assembly on one end and a motor assembly on the other. A launch lock that constrains the motion of the detector about the yaw axis during ascent is mounted to the inside of the yoke and attaches to a flange that is mounted on one of the detectors. The yoke is suspended between two aluminum pylons by another pair of motor/encoders. The pylons are bolted to an aluminum I-beam baseplate. The baseplate is attached to the spacecraft with eight $3 / 8$ in titanium bolts (four on each side). Pointing of the detectors is achieved through the two motor/encoder assemblies. The yaw motor/encoder assembly pivots the detector inside of the yoke while the pitch 
motor/encoder assembly pivots the yoke assembly between the two pylons. The pylon/baseplate system (Figure 2.5, Table 2.2) consists of four primary components:

1. Two pylons constructed from 0.25 in $6061 / \mathrm{T} 6$ aluminum plate forming a box structure. Each pylon is bolted to the baseplate with six 5/16 in stainless steel bolts and two flange assemblies. The flange assemblies tie the pylon outside plate to the baseplate in two locations.

2. One baseplate constructed from 6061/T6 aluminum I-beams. The baseplate is bolted to the space vehicle through eight $3 / 8$ in titanium bolts. The launch lock that constrains the yoke in motion about the pitch axis during ascent is mounted in the center of the baseplate.

3. One central electronic box radiator (CE Box) that is integrally attached to the $+Y$ side pylon. The CE Box is bolted to the baseplate and the radiator. The radiator, in turn, is bolted to the pylon.

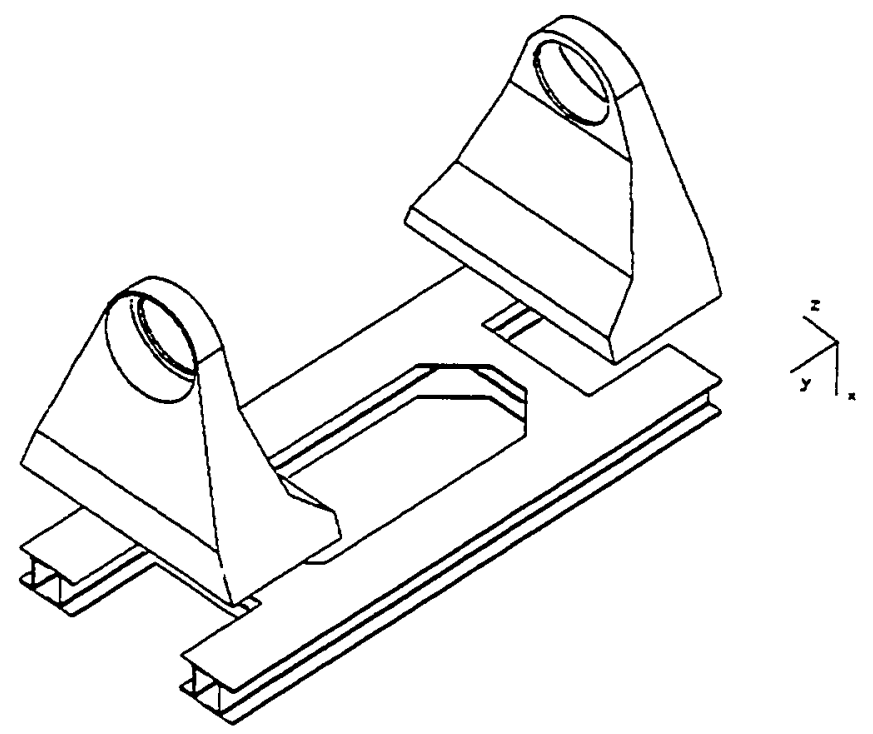

Figure 2.5: Unconventional Stellar Aspect Experiment Pylon/Baseplate Assembly 


\subsection{USA Structural Design and Analysis}

The design of the USA gimbal structure is governed by the P91-1/USA Interface Control Document (ICD, included in Appendix A). This document specifies the design environment in terms of expected external loads and sets the design performance requirements. The following is a list of design issues pertinent to the USA structure:

1. The lowest vibration mode of the structure in the launch condition must be greater than $50 \mathrm{~Hz}$ in frequency. This allowed the spacecraft design to proceed by modeling the experiments as rigid bodies, thus alleviating the need for the experimenters to deliver extensive finite element models early in the program.

2. All structural members must be designed with a Factor of Safety (FOS) of 2.0 if no static load testing is performed. Members that are to be static load tested must be designed with a FOS of 1.25. (See Tables 2.3 and 2.4) 3. A positive margin of safety under launch loads must exist on all structural members. This must be demonstrated through analysis and/or testing.

4. Clearance between the detector and yoke and between the yoke and pylons and baseplate must be maintained throughout their motion.

5. Launch locks must be positioned to provide high stiffness at launch while allowing full motion of the assemblies after their retraction.

6. The central electronics box must be positioned inside one of the pylons.

7. The structure must fit inside the envelope specified in the USA ICD.

8. Access to the central electronics box, the motors, encoders and feedthroughs must be provided.

9. Final assembly of the structure should be made simple as possible.

10. Each motor/ encoder pair should be co-aligned and the lines of action of the two pair should be normal to each other in the assembled system.

11. Weight and cost of the structure should be kept to a minimum. 


\begin{tabular}{|l|c|c|}
\hline & Axial (g's) & Lateral (g's) \\
\hline Lift Off & $+3.25 /-0.26$ & 4.0 \\
\hline Main Engine Cut Off & +7.1 & 0.13 \\
\hline
\end{tabular}

Table 2.3: USA ICD Static Flight Accelerations

\begin{tabular}{|l|c|c|}
\hline & Axial (g's) & Lateral (g's) \\
\hline Lift Off & $+6.5 /-0.52$ & 8.0 \\
\hline Main Engine Cut Off & +14.2 & 0.26 \\
\hline
\end{tabular}

Table 2.4: USA Design Flight Accelerations

A preliminary structural design for the USA experiment was performed at NRL using design techniques that are standard in the aerospace community. This called for machining the yoke and pylons from solid pieces of 6061/T6 aluminum plate. This design was accompanied by a finite element model run on NASTRAN that verified its acceptability as a preliminary design. The model was transferred to SLAC (along with the responsibility for the analysis and design of the supporting structure) and ported into CAEDS. CAEDS is a standard CAD package written by SDRC Corporation and supported on IBM RS6000 machines. It provides an excellent platform for both finite element modeling (FEM), solid modeling and drafting. The solid modeling and drafting capabilities would later prove to be critical to the manufacturing of USA.

An initial analysis of this model indicated that the primary design driver is the minimum natural frequency requirement $(>50 \mathrm{~Hz})$. Additionally, the primary static load is due to the axial component of the acceleration (in the $Z$ direction, along the direction of launch) of the detector in the yoke. This load causes a bending load in the yoke and is passed to the pylons through the motor and encoder to the pylons. The pylons are then subject to a combination of compression and bending. The lateral component of acceleration san act in any direction in the $\mathrm{YZ}$ plane. This will cause a combination of bending and twisting of the yoke and a bending and twisting of the pylon depending on the direction of the acceleration. 
Several different yoke cross-sections were modeled. These included the baseline C-section (NRL), a T-section and an I-section. Each design was kept to a constant total weight. The performance of the design in terms of the static load and natural frequency were compared to the baseline design. It should be noted that the NRL designed yoke was limited to a $\mathrm{C}$-section with external access by machining requirements. The depth of cut necessary to machine an I-beam cross-section ( $\sim 5$ in) was not feasible at a reasonable price. Furthermore, a $\mathrm{C}$-section opening to the inside of the yoke would make machine tool access very difficult, driving up the manufacturing costs. However, the design survey indicated that the most efficient cross section for the yoke was an I-beam with the web lying in the XY-plane. Furthermore, it was found that by closing the I-beam section at the four corners of yoke, the box structure formed would greatly increase the torsional stiffness of the structure (Figure 2.6). This could be done by placing two additional webs between the inner and outer flanges at each comer. This additional stiffness was not necessary for the preliminary design to meet the specifications set in the ICD.

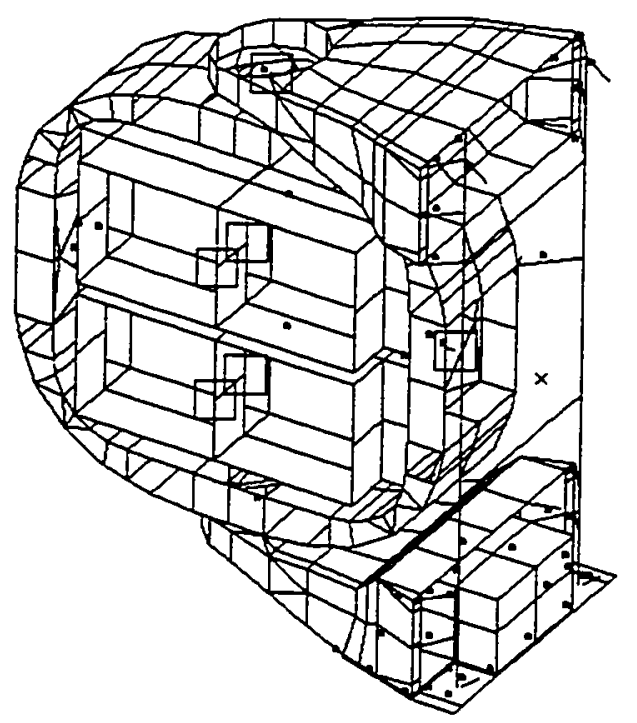

Figure 2.6: Unconventional Stellar Aspect Experiment Finite Element Model As the overall USA design became better defined, we suffered a weight growth of approximately $25 \%$ from the estimated masses to the as-manufactured masses. it then 
became necessary to include the box structure in the design to meet the natural frequency requirement. This closed box structure made machining the yoke from a solid block of aluminum even more impractical. Three alternate methods were devised to manufacture the yoke: bolted structure, welded structure, and a dip-brazed structure. The approach of using several small pieces bolted together was rejected for three reasons: such structures would be difficult to analyze, inefficient in their use of weight and would make it difficult to maintain close tolerances on the motor mount locations without expensive jigging. The fact that this structure contained precisely aligned motors and encoders acting along common axes meant that the locations and alignments of the motor and encoder mounts had to be placed as accurately as possible. The thermal deformations associated with a welded structure were deemed to be too great to use this technique. Welding also requires extensive testing and qualification for use in manufacturing space qualified structures.

It was decided to manufacture the support assembly with a process called dipbrazing (Figure 2.7). Dip brazing has been used for 30 years to bond fairly small parts together where a direct contact is necessary, but has not been used extensively to manufacture space qualified structural components (Altshuller, et al, 1990). With this technique a bond is formed between two pieces of aluminum as follows:

1. The bond surfaces of the joints are cleaned and prepared.

2. Braze material is inserted into the joints and the joints are held together by tack welds. The braze material has a melting point that is typically 50 of lower than the parent material.

3. The assembly is preheated.

4. The assembly is dipped in a bath of molten salt. This heats the joints to a level that melts the braze material and causes it flow, forming the bond.

5. The assembly is removed from the bath and quenched.

6. The assembly is heat-treated. 


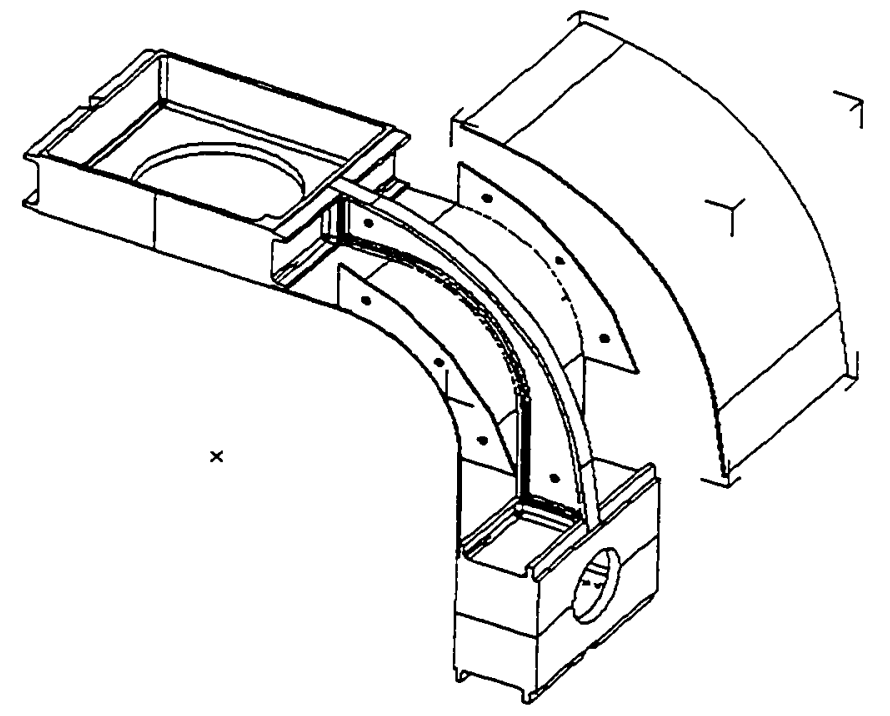

Figure 2.7: Unconventional Stellar Aspect Experiment Yoke Assembly

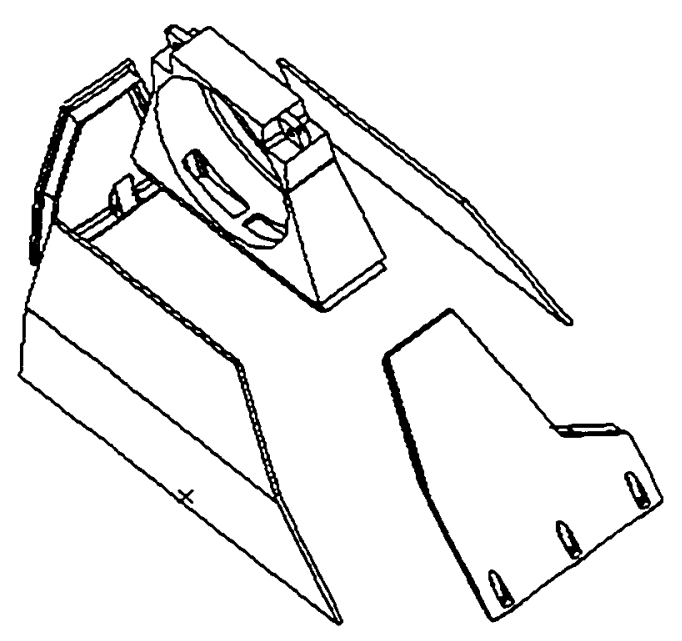

Figure 2.8: Unconventional Stellar Aspect Experiment Pylon Manufacturing Assembly

Because the dip-brazing process is fairly new to the aerospace community and no one within the collaboration, at the Aerospace Corporation or at Rockwell had any 
experience building large assemblies with it, a conservative approach to manufacturing was used. This included the following elements:

1. Large margins in the strength of the structure were maintained, especially at the beginning of the program.

2. The dip-brazing vendor was qualified before the flight articles were constructed.

3. A conservative approach to the design of the braze joints was employed by using large faying surfaces and interlocking parts at all critical joints.

4. A prototype pylon was constructed and static load tested to $125 \%$ of the expected worst-case flight load.

5. A structural test program was implemented that was integral to the manufacturing process (see below).

The pylons were also constructed by using the dip-brazing process (Figure 2.8). The design of the pylons was governed primarily by ease of manufacturing and ease of access questions. The $+Y$ side pylon has to contain and support the central electronics box and its accompanying external radiator. Both pylons have wire harnesses that run up to the motor ( $+Y$ side) and encoder ( $-Y$ side). Therefore both pylons have to provide easy access during assembly and testing. In order to minimize the cost of the pylons and to allow easy access internally, a box construction was used. The side pieces were machined from 1 in $(25 \mathrm{~mm})$ aluminum plate stock while the front and back were made from 0.25 in $(6 \mathrm{~mm})$ aluminum plate. A bolted cover piece was provided on both pylons.

The dip-brazed design had a $20 \%$ reduction in structural weight over the original design and cost about $\$ 35,000$ to manufacture and test compared with the $\$ 80,000$ estimate for an integrally machined design. 


\subsection{USA Structural Test Program}

below:

The testing program that was applied to the USA experiment is summarized

1. Construction to established specifications:

All dip-brazing was done by Aluminum Dip Braze Co. (ADB) in Burbank, Ca. This work was done in accordance with Mil-B-7553-B Type V Grade B with quality standards per Mil-I-45208. All joint preparation was done to Mil-B-7553-B Type V Grade A specifications. Following the dip-brazing process, all parts were heat-treated to attain T6 conditions. All dip-brazed joints were then inspected by radiographic methods and the results interpreted to determine the fractional braze wetting of each joint (Appendix B). Certifications of Conformance are on file with radiographs for all parts. I was present to videotape the brazing of the prototype pylon and the yoke.

2. Manufacturing qualification:

A series of qualification test samples were built by $\mathrm{ADB}$ and tested to failure at SLAC. This work is described in detail in Appendix B.

3. Prototype pylon and flight articles:

After qualifying the manufacturing process, one prototype pylon was manufactured by $\mathrm{ADB}$ to prove the overall manufacturing concept. This pylon would later be subjected to a static load test. Only after visual and radiographic inspections of the prototype pylon were performed were the flight articles manufactured. The flight articles included two pylons and one yoke.

\section{Quality assurance pull samples:}

Each item dip-brazed by ADB was accompanied by five shear samples similar to those used in the manufacturing qualification process. These samples were prepared in the same manner and to the same specification as the prototype and flight articles and were brazed in the same bath and at the same time as their parent articles. The results of these tests are described in Appendix B. 


\section{Static Load Test}

As a final test of the USA structure, the prototype pylon was used in a static load test that subjected the pylon to $125 \%$ of the USA ICD loads. This test is described in detail in Appendix B.

\subsection{USA Thermal Analysis}

An important aspect in the design of any satellite is its response to the thermal environment on orbit. Many components of a spacecraft (electronics, batteries, etc.) must be kept within strict temperature bounds in order to ensure their reliable operation. In order to keep the operating temperatures of these components below the design maximum, the heat generated internally must be given proper paths to flow to specially designed radiators that then radiate that heat to outer space. This is in addition to the significant heat from the sun and earth that is absorbed by all surfaces of the vehicle. To complicate matters, there are scenarios where the power to certain non-essential systems is turned off. In these survival modes, it is necessary to supply survival heat to sensitive components to keep them from getting too cold. The design of the thermal control system requires a detailed model of the spacecraft and simulations of its operation on orbit in all possible scenarios.

While several software packages exist that solve time varying thermal analysis problems, none of these were available to the USA project. So, preliminary thermal design studies were conducted using software that I wrote in MATLAB. The initial versions of this software solved the static case where all temperatures are constant and the thermal inputs are averaged over the orbit. By first considering the static case, I was able to get estimates for survival heat and radiator areas that were within $10 \%$ of the final design. The software package was later expanded to the time varying case by integrating the governing equations with time varying heat inputs. This complete package was used to finalize the USA thermal design.

The thermal control system for the USA experiment uses a combination of thermal radiators and survival heaters to maintain the temperature of critical components 
within strict bounds (Figure 2.9). The only variables open to the designer are the area of the radiator, the amount of survival heat, and, to a lesser extent, the optical properties of the surfaces of the experiment. The basic thermal design is summarized in Table 2.3.

\begin{tabular}{|l|c|c|c|c|c|}
\cline { 2 - 6 } \multicolumn{1}{c|}{} & \multicolumn{2}{c|}{ Power Dissipation (W) } & \multicolumn{2}{c|}{ Design Temperature (C) } & Radiator \\
\hline Component & Operational & Survival & Minimum & Maximum & Area $\left(\mathrm{m}^{2}\right)$ \\
\hline CE Box & 35 & 23 & -29 & +50 & 0.15 \\
\hline Motors (2) & $1(\mathrm{ea})$ & 0 & -125 & +125 & 0.00 \\
\hline Encoders (2) & $0.5(\mathrm{ea})$ & 0 & -125 & +125 & 0.00 \\
\hline Detector & & $10(\mathrm{ea})$ & -29 & +45 & $0.05(\mathrm{ea})$ \\
\hline Electronics (2) & $10(\mathrm{ea})$ & 0 & -10 & +40 & 0.00 \\
\hline Viton Seal (2) & 0 & 0 & -50 & +250 & 0.00 \\
\hline Heat Shield (2) & 0 & & 0 & & \\
\hline
\end{tabular}

Table 2.5: USA Thermal Design

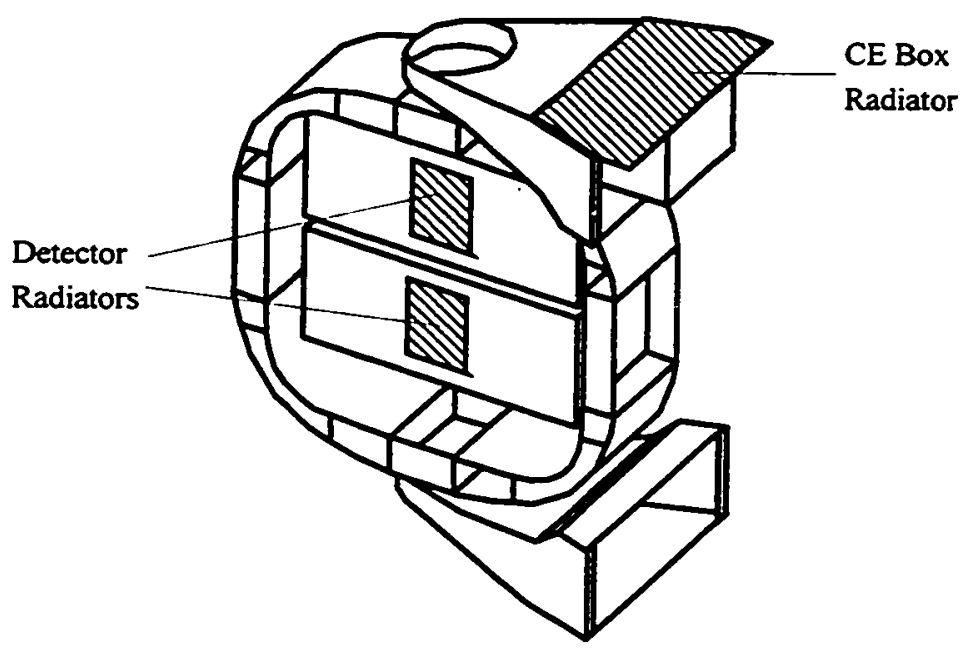

Figure 2.9: USA Thermal Control System 
For the purpose of thermal analysis, the USA experiment can be broken into three independent models: the detector assembly, the yoke assembly and the pylon/baseplate assembly. The detector assembly consists of the two proportional chambers and their accompanying collimators, electronics, and gas systems and the detector frame to which they are mounted. This assembly is mounted inside the yoke via a stepper motor and encoder such that it can rotate freely about the local "yaw" axis. The yoke is an aluminum assembly that is in turn mounted between the pylons of the pylon/baseplate assembly via another stepper motor and encoder to provide rotational freedom about the spacecraft pitch axis. The conductive paths connecting the detector and pylon assemblies to the yoke travel a circuitous route through the motor and encoder assemblies. The thermal resistance along these paths is quite large and allows the three models to be considered independently. These models are described pictorially in Figures 2.10, 2.11 and 2.12.

All components of the USA experiment are covered completely in multi-layer insulation, with the exception of specific radiating areas and the detector aperture. This insulation has a blackened kapton outer sheet. The MLI is assumed to have an effective emissivity of 0.01 . The effects of the external optical properties of the MLI on the heat leak through the insulation are negligible when compared with the effective emissivity. That is, while the optical properties of the blanket's outer sheet may reject $20 \%$ of the incident radiation, the layered properties of the MLI alone rejects $99 \%$. The radiators are covered with a silver-teflon tape that provides a high emissivity in the infrared bands $(\sim 0.80)$ for efficient radiation of internal heat but a low absorptivity in optical bands $(\sim 0.20)$ for good rejection of incident radiation. There are three such radiators - one on the back of each detector and one on the central electronics box. The detector apertures are covered with second surface aluminized mylar heat shields. These insulate the detectors while absorbing a minimum of incident low energy $x$-rays.

Survival heater power is provided to the electronics of each detector and the central electronics box from the spacecraft main power bus. Each survival heater is governed by a thermostat. 


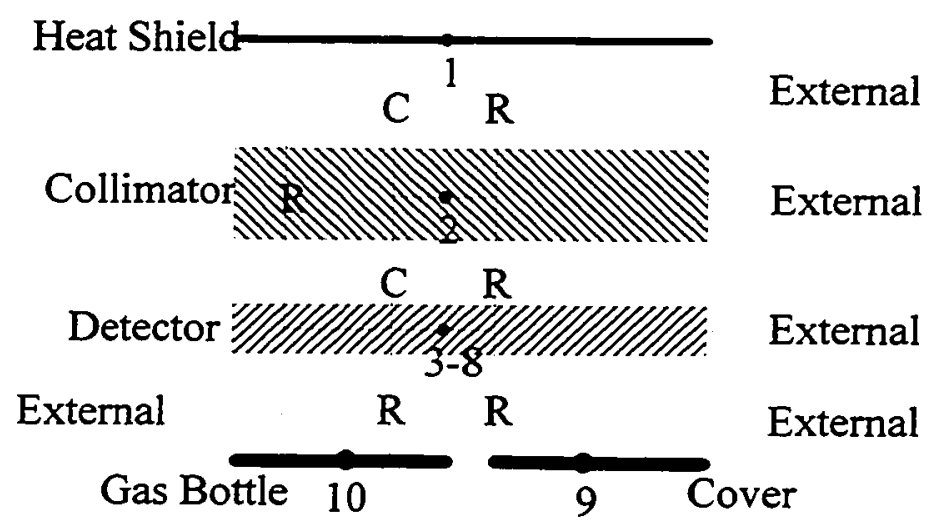

Figure 2.10: USA Detector System Thermal Model

y

$\mathbf{x}$

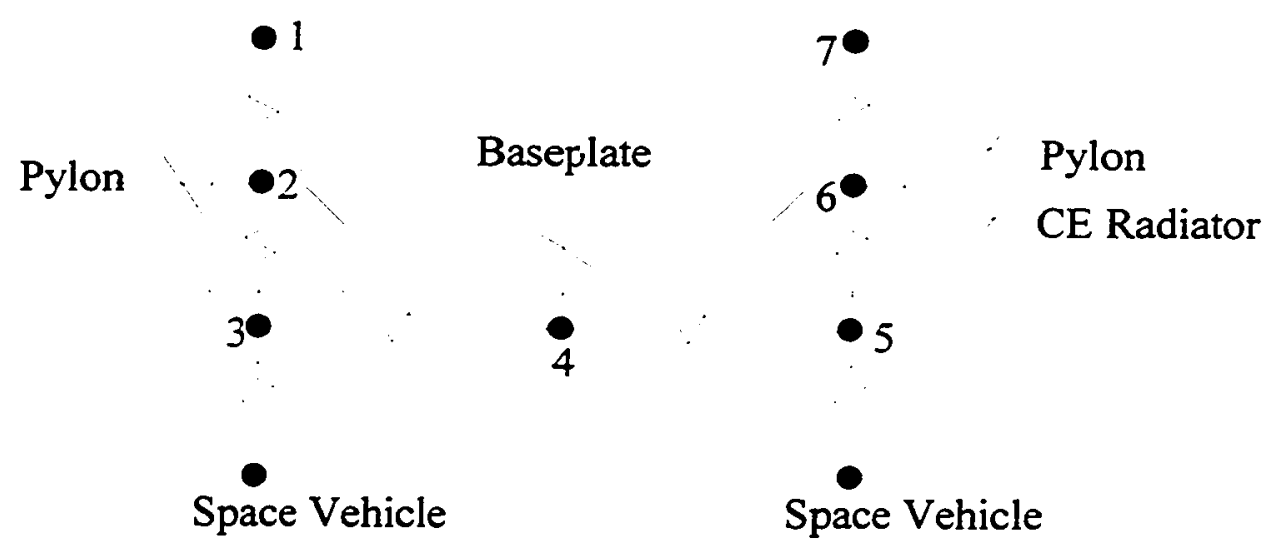

Figure 2.11: USA Pylon/Baseplate Thermal Model
Radiation Connection Conduction Connection 
Y

\section{Conduction}

$\mathbf{X}$

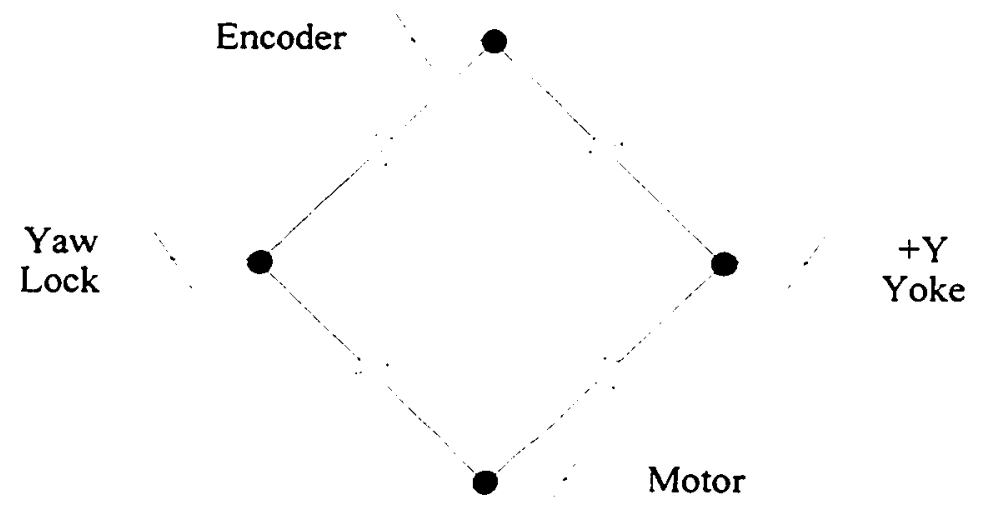

Figure 2.12: USA Yoke System Thermal Model

The pylon/baseplate system was modeled with the following assumptions:

1. The interface resistance between the pylons and the baseplate was neglected in this model. Both pylons are covered in multi-layer insulation with a blackened kapton outer surface.

2. The baseplate is bolted to the space vehicle through eight $3 / 8$ in titanium bolts. The interface resistance of these bolts was modeled by two conductive elements, one on the $+Y$ side of the experiment and one on the - Y side. The baseplate is covered in multi-layer insulation with a blackened kapton outer surface.

3. The central electronic box radiator (CE Box) is integrally attached to the $+Y$ side pylon. Heat dissipated by the electronics in operational and survival modes is assumed to be dissipated directly into the radiator. The external radiator surface is covered with silver teflon tape. Radiative heat 
transfer between the bottom of the CE Box and the spacecraft interface was neglected. These surfaces will be covered with low emissivity materials to eliminate such heat transfer. The thermal design for USA used the temperature of the radiator as the design goal for the central electronics box temperature.

4. The pylon/baseplate system is broken into three diffusion and 5 arithmetic nodes.

5. In the calculation of the conductivities between pylon nodes, the area used to calculate the conductivity between two nodes is the average crosssectional area of the conduction path between those nodes.

A simple model was created to describe the yoke system. It consists of four diffusion nodes representing the four quadrants of the yoke. The entire yoke is covered in $\mathrm{MLI}$ and it has no radiators. Again, in order to calculate the conductivity of the yoke between nodes, the average cross-sectional area along the conduction path was used.

The USA detector system, consisting of two proportional chamber subsystems suspended in an aluminum frame, is modeled with the following assumptions:

1. All external surfaces of the proportional chamber and collimator frame are covered with MLI.

2. The aluminum detector cover covers the entire backplane of the detector. The external surfaces are covered with $\mathrm{MLI}$, with the exception of a single paich of radiator area that is covered with silvered teflon tape. The inside of the cover is radiatively coupled to the detector backplane. 
3. The gas bottle is completely covered in MLI and contains no internal heat generation. It is connected to the proportional chamber via a small bolted surface.

4. The conductive resistance of the mylar window and heat shield was neglected. All heat absorbed by the window is reradiated as a diffuse gray body.

5. The collimator frame and collimators were lumped together as a single diffuse node. They include a conductive path to the detector body as well as radiative paths between the heat shield and the window.

6. The heat shield was modeled as a second surface reflector with optical properties provided by Swales Associates.

7. The detector body is modeled with five diffusion nodes with conductive and radiative connections between them.

There are three operational modes on the ARGOS satellite - initial deployment. operational and sunsafe. Each of these modes presents a different set of heat inputs and boundary conditions to the USA experiment. In the initial deployment mode (Figure 2.13a), the satellite is in its nominal orbit configuration. The $+Z$ axis is nadir pointed and the $\mathrm{X}$ axis is in the velocity direction. The USA detectors and electronics are turned off, but the survival heaters are turned on to keep the temperatures of sensitive components within specifications. Throughout initial deployment the $+Y$ side of the vehicle never sees direct sunlight.

A few weeks into the mission the USA experiment will go from initial deployment mode to operational mode. In this mode the satellite is in the same nominal configuration as in initial deployment, but the experiment is free to rotate inside the pylons. Additionally, the detectors and electronics are turned on. While the power to the 
survival heater buses is never turned off, the heaters are controlled by thermostats located on critical USA components. During USA operations, the experiment should never get cold enough for the survival heaters to be turned on.

A special survival or sunsafe mode has been provided on the ARGOS spacecraft (Figure 2.13b). In this mode the satellite configures itself for maximum power generation and minimum power consumption. The satellite is inertially stabilized with the solar arrays pointed directly at the sun. This places the $-Z$ axis $30^{\circ}$ off the sunline. The operational power to the experiments is turned off and the survival heaters are left on. 


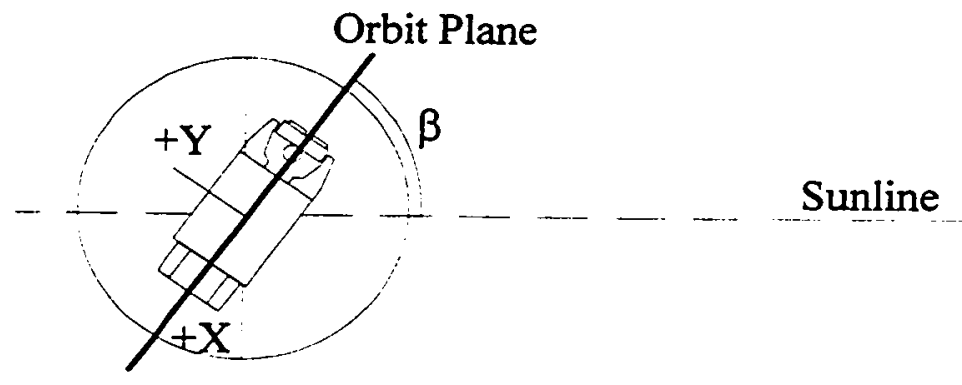

Figure 2.13a: USA Initial Deployment/ Operational Mode

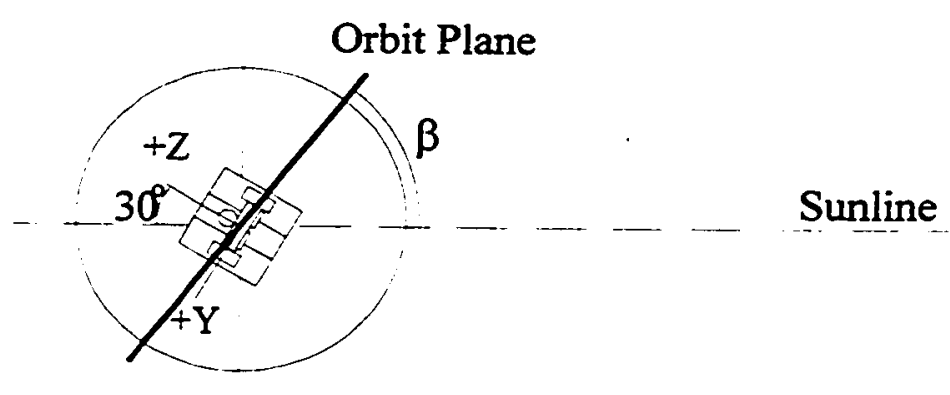

Figure 2.13b: USA Sunsafe Mode 


\section{Chapter 3: X-ray Attitude Determination}

\subsection{Introduction}

The orientation of a spacecraft relative to an inertial frame commonly referred to as the celestial sphere can be determined by measuring the location of one or more guide stars in the spacecraft frame. It is common practice to make these observations using instruments sensitive to guide star emissions in the optical or ultraviolet light wavelengths. These measurements can also be made by using detectors sensitive to the $\mathrm{x}$-ray light emitted by many of the same guide stars.

Two such instruments will be considered here. An x-ray star mapper takes a single image of a large portion of the sky and locates several guide stars in the image. The pattern of guide stars is compared to an on-board catalog to determine the spacecraft orientation. An x-ray star scanner measures the location of each guide star independently by scanning an $x$-ray detector over each star successively.

There are several advantages to using the $x$-ray sky as an inertial reference. The small number of bright sources emitting in the $x$-ray regime means that the number of stars in an on-board catalog need not be very large. This results in a reduction in the 
instrument memory requirements needed to store the catalog. The instrument computing requirements are also reduced since the complexity of the image matching routine is lessened. The complexity of an x-ray star scanner is also reduced since fewer bright sources generally means less source confusion. Additionally, the temporal variabilities (pulsations) that are present in the intensities of many $x$-ray sources can be used during attitude initialization to identify a crude pointing direction. This can also be used during the image matching process to explicitly identify specific sources in an image, further reducing the complexity of the image matching process.

\subsection{X-ray Star Mappers on Pointed Satellites}

\subsubsection{Missions and Methodologies}

Star mappers have traditionally been used for missions where pointing knowledge requirements are very strict - as tight as arc-second resolution. Recent designs of these instruments have involved using a lens system and a planar CCD array to image the optical or ultraviolet star field in detector coordinates. A comparison of bright sources in the image to a catalog of guide stars by one of several image matching algorithms results in a measure of the inertial attitude of the spacecraft. A great deal of effort has been put into developing efficient image matching routines. These generally involve measuring the angular displacements in the image between pairs or triplets of guide stars. These measurements are then compared to the known angular displacements between a large number of guide stars to determine the orientation of the spacecraft (van Bezooijen, 1992). The high performance of these instruments is accompanied by several drawbacks. They tend to be expensive, heavy and require a lot of power when compared to other attitude sensors. Furthermore, bright sources other than the guide stars (sun, earth, moon) can introduce errors in the measurement process. The image system can even be damaged if care is not taken to keep such sources from shining directly on the collecting area of the instrument. This generally involves placing a large sun shield in front of the star mapper to limit its field of view and constraining the attitude motion of the vehicle to 
keep bright sources from passing in front of the sensitive instrument. The detectors used to collect $\mathrm{x}$-rays can operate effectively in such harsh environments and can withstand very high count rates (e.g. from the sun) without being permanently damaged (electronics sensitive to high count rates can be shut off before damage occurs). X-ray star mappers can be used without special constraints on the attitude motion of the spacecraft since they will not be damaged by viewing bright sources.

There are three ways to create an $\mathrm{x}$-ray image:

1. By measuring the direction of each incoming photon directly.

2. By using a lens or mirror system with a position sensitive detector.

3. By using a coded mask with a position sensitive detector.

Detectors capable of measuring the direction of a photon directly are effective at detecting only high energy $x$-rays and $\gamma$-rays. The low count rates associated with sources at such energies makes them undesirable for use as an inertial pointing reference. The collecting area required for a reasonable signal-to-noise ratio and measurement bandwidth would be quite large (i.e. tens of square meters).

Grazing incidence mirrors have been used in conjunction with position sensitive $x$-ray detectors to image the $x$-ray sky. While an instrument with grazing incidence mirrors and a pixelated detector offers a direct and continuous image of the x-ray sky, these mirrors tend to be large, heavy and expensive.

Using coded masks to image the $x$-ray sky was first proposed by Dicke in 1961 (Dicke, 1961). These systems use a pixelated detector with a mask that has a pseudorandom pattern of holes in it. The image returned by the detector is then a convolution of the actual $x$-ray sky and the coded mask pattern. The literature on the design of these masks is quite extensive (Fenimore, 1978; Massen, 1987). A number of reconstruction schemes have been demonstrated for deconvolving the detector image into an image of the $x$-ray sky (Skinner, 1984). This image can then be compared to an onboard catalog of $\mathrm{x}$-ray guide stars with the same methods as those developed for optical star mappers. While these instruments are relatively easy to manufacture, they do have 
one drawback. The process of deconvolving the detector image introduces errors in the reconstructed image that would not be present when using direct imaging systems. Typically, these errors appear as ghost images and are due to the fact that there are an infinite number of solutions to the reconstruction problem.

\subsubsection{Pointing Feasibility Study}

In order to show that an $x$-ray star mapper can be used as a precision pointing instrument, a simple $x$-ray imaging system is considered. This instrument is based on a proposal by Dr. Kent Wood for an $\mathrm{x}$-ray imaging system called the Silicon Imaging $\mathrm{X}$-ray Instrument (SIXI) that will demonstrate the performance of an x-ray star mapper as a precision attitude reference. The resolution of this instrument is fairly moderate at $0.05^{\circ}$ and its collecting area is roughly $0.36 \mathrm{~m}$ on a side. The SIXI mission will use a coded aperture system to generate the images. Assuming a coded mask that occludes $50 \%$ of the detector area. the effective collecting area of this instrument is reduced by a factor of two to $0.0648 \mathrm{~m}^{2}\left(648 \mathrm{~cm}^{2}\right)$. Such an instrument could be used on an inertially pointed or slowly spinning (e.g. nadir pointing) spacecraft. The following assumptions are made in this analysis:

1. An $\mathrm{x}$-ray imaging device is considered.

2. An image of the sky is taken in x-rays without imaging errors. In reality there will be errors (ghosts. blurring) in the image associated with imperfect lenses or the coded aperture reconstruction.

3. It is assumed that the jitter or spin of the satellite that occurs while taking an image is less than the resolution of the detector.

4. A simple attitude determination algorithm is used and will be discussed later.

5. The $x$-ray sources are assumed to be point sources. A few $x$-ray sources actually behave as diffuse sources several arc-seconds across. 
6. The image is "taken" with an instrument with the same effective energy response as the HEAO-Al instrument. That is. it is sensitive to photons with energies between I keV and $30 \mathrm{keV}$.

7. The HEAO-Al catalog is assumed to be a complete and accurate representation of the $\mathrm{x}$-ray sky. A map of the $\mathrm{x}$-ray sky corresponding to the sources in this catalog is shown in Figure 3.1.

8. The number of counts in the image bin corresponding to a particular source are defined by a Poisson distribution with a mean corresponding to the source intensity as indicated in the HEAO-Al catalog.

9. The instrument configuration is assumed to be similar to the SIXI mission. The relevant parameters are summarized in Table 3.1 .

\begin{tabular}{|l|l|}
\hline Field of View & Cone with $40^{\circ}$ half angle \\
\hline Attitude Solution Error Box & Cone with $2.5^{\circ}$ half angle \\
\hline Bin Resolution (Size) & $0.05^{\circ} \times 0.05^{\circ}$ \\
\hline \# Solution Bins (n) & 10.000 \\
\hline Energy Range & Approximately HEAO-A1 \\
\hline Detector Area & $648 \mathrm{~cm}^{2}$ \\
\hline Background Rate & 30 counts $/\left(\mathrm{sec}^{*} \mathrm{~cm}^{2}\right)$ whole sky \\
\hline
\end{tabular}

Table 3.1: Ideal Detector Parameters 


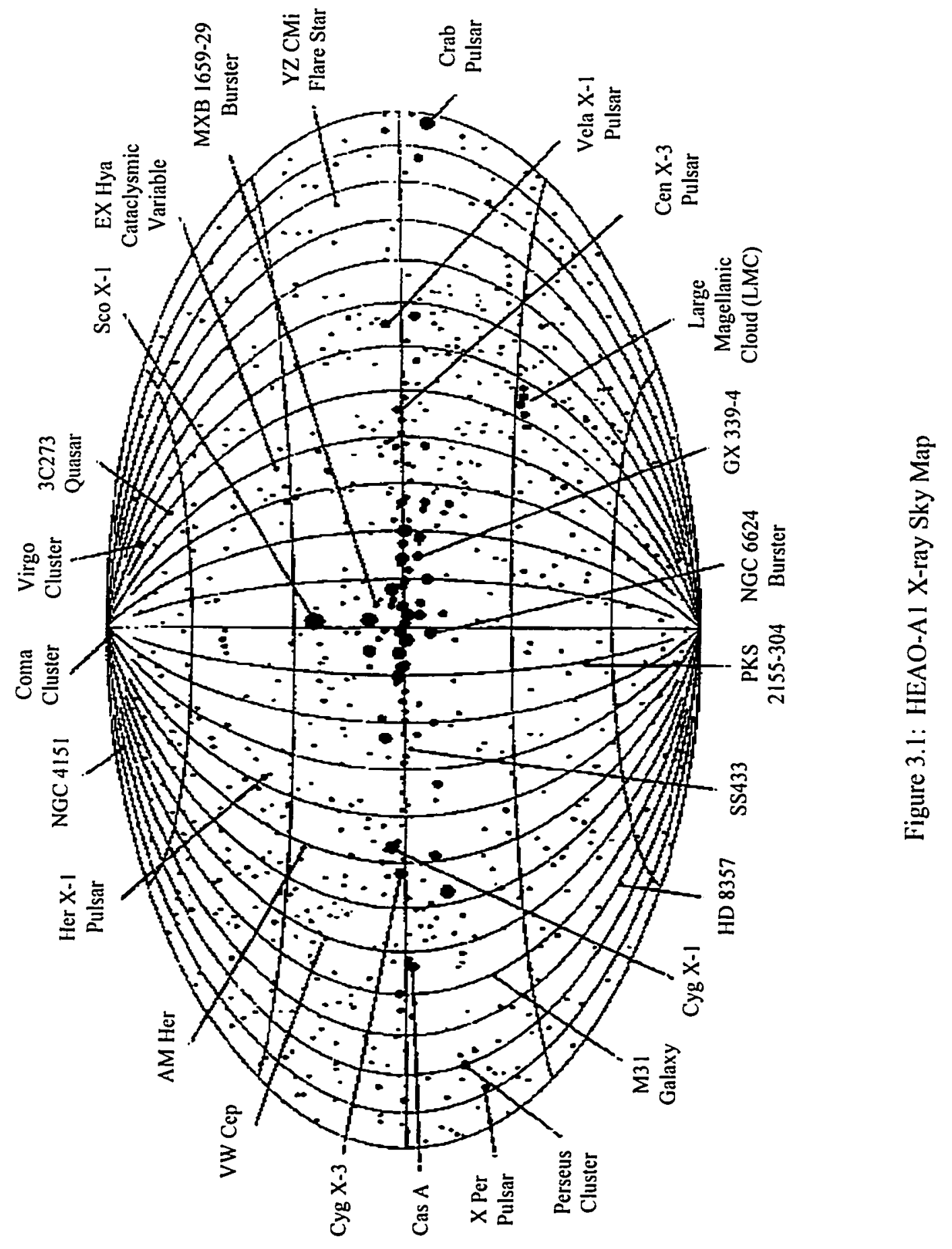


The attitude determination algorithm used in this study is as follows (see Figure 3.2):

1. Assume that an attitude fix has been made at time $t_{0}$.

2. Predict the attitude at time $t_{1}=t_{0}+\Delta t$ by some form of forward integration. This gives an expected field of view for the $x$-ray star mapper. 3. Search the on-board catalog for the brightest $x$-ray source in the expected field of view. This is the guide star.

4. Estimate the maximum possible error in the attitude prediction at time $t_{1}$. This gives the attitude solution error box that is centered about the guide star.

5. Collect $x$-ray photons for $t_{c}$ seconds to create an image of the $x$-ray sky.

6. Search the region of the $x$-ray image corresponding to the attitude solution error box for the brightest source in that box. If the exposure time is sufficiently long, the probability of a background bin in the image having more counts than the guide star bin is negligible.

7. The possibility of source confusion is neglected. It is assumed that a more complex algorithm can be used to alleviate this problem.

8. The position of the guide star in instrument coordinates can then be compared to the inertial position of the guide star from the on-board catalog to get an inertial attitude fix at time $t_{1}$. 


\title{
Initial Attitude
}

Fix Given

A0, to

\section{Estimate \\ Attitude \\ Al, $\mathrm{tl}$}

\section{Estimate}

Attitude Error

$\Delta \mathrm{Al}, \tau \mathrm{l}$

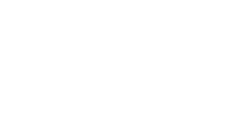

Select

Guide Star

from Catalog

\section{Determine}

Attitude Error

Box

Find Brightest

Source in Image

within Error Box

Image X-ray

Sky for te

\section{Determine \\ Attitude \\ Al, tl}

Attitude

Determination

No

Is Guide Star.

Brighest Spot

Yes

Attitude

FAILED

Figure 3.2: X-ray Star Mapper Feasibility Study 
The problem then is to compute the exposure time necessary to guarantee (to an acceptable probability) that the guide star will be the brightest source in the attitude solution error box. If the image of the sky is divided into a number of equal bins. then the number of counts in any bin is given by a Poisson distribution. Defining $X$ to be the number of counts in the bin containing the guide star and $Y_{i}$ to be the number of counts in the $i^{\text {th }}$ background bin, the number of counts in any bin are given by:

$$
\begin{array}{ll}
P(X=k)=e^{-\mu_{s} A t_{e}} \frac{\left(\mu_{s} A t_{e}\right)^{k}}{k !} & \text { (guide star bin) } \\
P\left(Y_{i}=l\right)=e^{-\mu_{b} A t_{e}} \frac{\left(\mu_{b} A t_{e}\right)^{l}}{l !} & \quad \text { (i }
\end{array}
$$

where.

$$
\begin{aligned}
& \left.\mu_{\mathrm{s}}=\text { Mean rate of guide star (counts } / \mathrm{sec}^{*} \mathrm{~m}^{2}\right) \\
& \mu_{b}=\text { Mean rate of background (counts } / \mathrm{sec}^{*} \mathrm{~m}^{2} \text { ) } \\
& A=\text { Instrument effective collecting area }\left(\mathrm{m}^{2}\right) \\
& t_{c}=\text { Instrument exposure time (sec) }
\end{aligned}
$$

The probability of successfully determining the attitude is the probability that the image bin of the guide star contains more counts than any other bin in the solution error box ( $\mathrm{n}$ bins total in the solution error box). That is.

$$
\begin{aligned}
& P(S)=P(\text { success })=P(\mathrm{X} \text { is maximum }) \\
& P(S)=\sum_{k=1}^{\infty} P(X=k) P\left(y_{1}<k\right) P\left(y_{2}<k\right) \ldots P\left(y_{n-1}<k\right) \\
& P(S)=\sum_{k=1}^{\infty} P(X=k)[P(y<k)]^{n-1} \\
& P(S)=\sum_{k=1}^{\infty} P(X=k)\left[\sum_{l=0}^{k-1} P(y=l)\right]^{n-1} \\
& P(S)=\sum_{k=1}^{\infty} e^{-\mu_{s} A t_{e}} \frac{\left(\mu_{s} A t_{e}\right)^{k}}{k !}\left[\sum_{l=0}^{k-1} e^{-\mu_{b} A t_{e}} \frac{\left(\mu_{b} A t_{e}\right)^{l}}{l !}\right]^{n-1}
\end{aligned}
$$


This equation can be solved numerically for $t_{e}$ given a series of source strengths. $\mu_{\mathrm{s}}$ a desired probability of success (in this case. $\mathrm{P}(\mathrm{S})=99.7 \%$ ), and the instrument parameters given in Table 3.1. The relationship between guide star intensity and required exposure time is plotted in Figure 3.3. This information is then used in conjunction with the HEAO catalog to generate a contour plot of the exposure time necessary to achieve an attitude solution as a function of the pointing direction of the $\mathrm{x}$-ray star mapper (Figure 3.4). The abscissa and ordinate in Figure 3.4 correspond to the galactic longitude and galactic latitude of the instrument boresight during a given observation. For each observation, the HEAO catalog is searched for the brightest source in the instrument field of view and it is selected as the guide star as previously discussed. The intensity of the guide star $\left(\mu_{\mathrm{s}}\right)$ is corrected for the effect of reduced detector area when the photons from the guide star impacted the detector at an angle by multiplying by the cosine of the angle between the instrument boresight and the line of sight to the guide star.

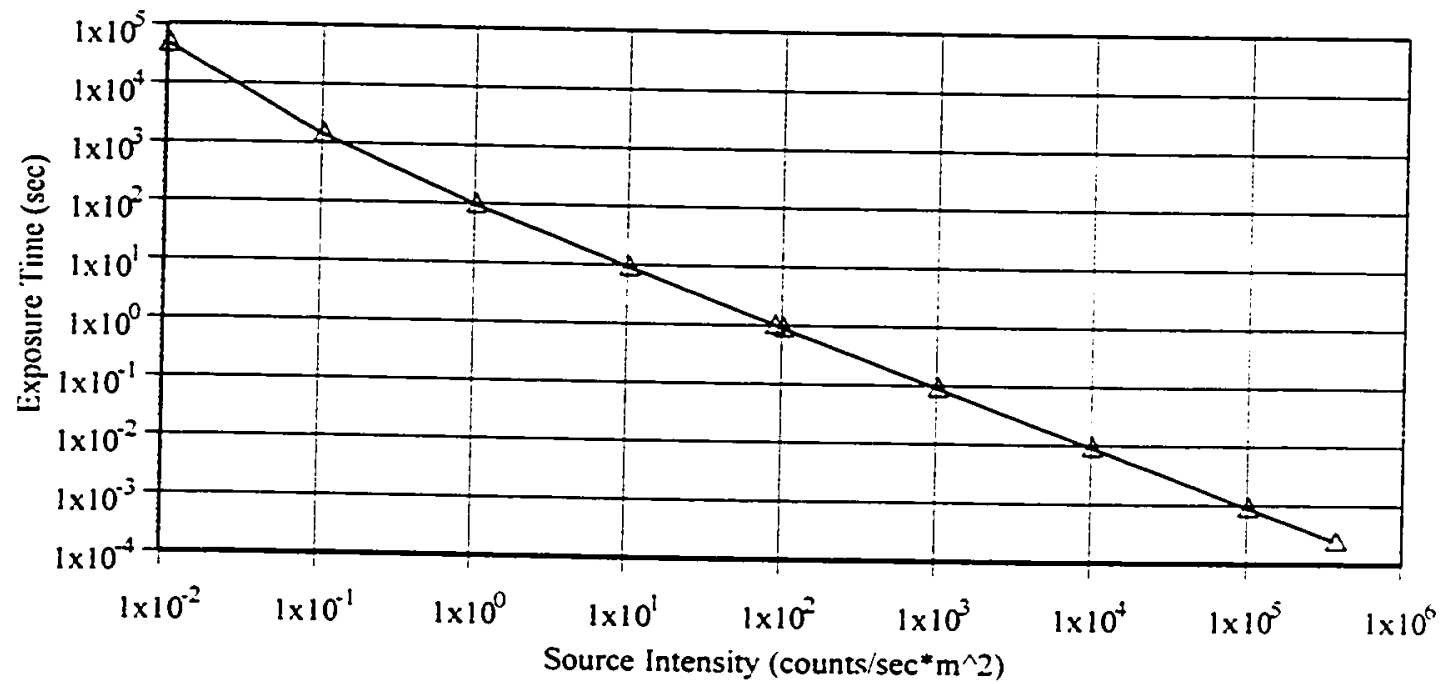

Figure 3.3: Ideal Star Mapper Sensitivity 


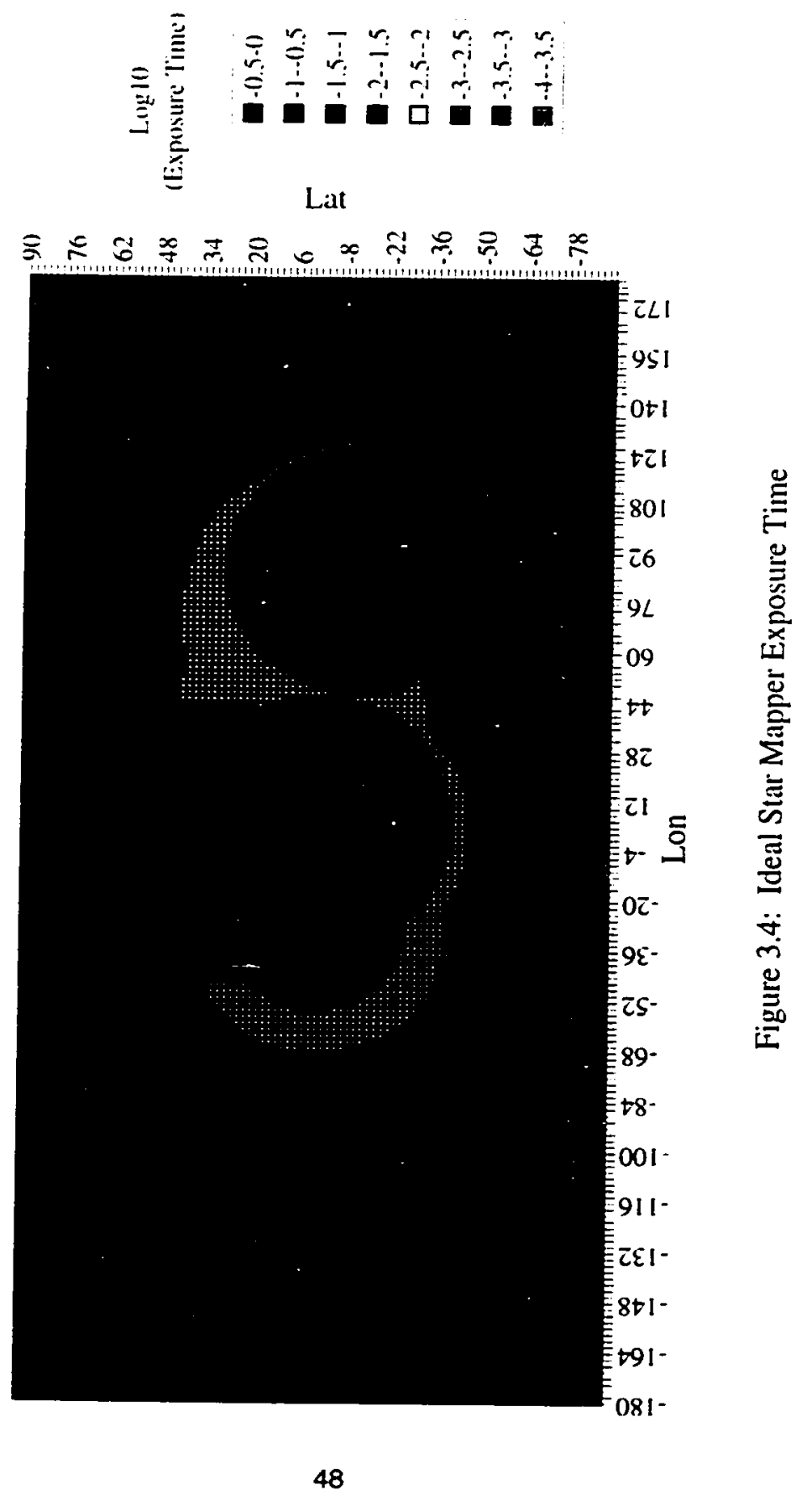


The results of this study suggest that for an x-ray star mapper with characteristics similar to the SIXI instrument, a minimum exposure time of about $1 \mathrm{msec}$ will be necessary when the brightest guide star (Sco-X1) is in the field of view. The required exposure time increases to about $10 \mathrm{sec}$ in areas where the guide stars are fainter. Note that the exposure time necessary to make a measurement is inversely proportional to the collecting area of the instrument. Thus, by doubling the instrument area, the exposure time can be cut in half. doubling the bandwidth of the instrument. The bandwidth of the attitude determination system can also be increased by using more than one instrument. each pointed in a different direction. When one instrument is looking at a portion of the sky where the guide stars are dim. the other instrument will see brighter guide stars allowing it to make measurements at a faster rate.

\subsection{X-ray Star Scanners on Spinning Spacecraft}

Spacecraft that spin about their maximum principal axes of inertia fill an important role in the design of spacecraft. These vehicles have two advantages: the location of the spin axis is relatively stable in inertial space and the spinning action of the spacecraft makes it an excellent scanning platform for observations of the earth and space. The stability of the spin axis makes this an inexpensive way of stabilizing a satellite when compared to a satellite that is controlled about all three axes. Measuring the attitude of a spinning satellite has been done with combinations of sun sensors. earth horizon sensors, star scanners and gyroscopes.

Using an $x$-ray star scanner is an excellent way to make accurate measurements of the orientation of a spinning spacecraft. This instrument is of simple design. consisting of a detector and a collimator and some associated electronics. The detector records the number of photons from the guide star as a function of time. The collimator attenuates the guide star intensity as a function of the orientation of the spacecraft. This effect is called the collimator transmission. The spinning action of the spacecraft causes the detector to "map" the collimator transmission as a function of time. making it possible to 
determine the orientation of the spacecraft. The sparsity of celestial objects radiating brightly in the $x$-ray regime makes an $x$-ray star scanner very appealing for use on spinning spacecraft. A star scanner has the advantage that the spinning action of the spacecraft causes guide stars to come into the field of view (FOV) of an instrument that has a relatively small inherent field of view. The FOV is effectively 360 degrees in the spin direction (normal to the spin axis). Instruments on satellites in planetary orbits would be limited to something less than a 360 degree FOV by the presence of the planet. but would certainly have a FOV greater that 180 degrees. Thus, the high signal to noise ratios associated with a small FOV instrument will be available to an instrument with a large FOV. The small number of potential guide stars $(<100)$ minimizes the potential for two guide stars to be in the instrument FOV simultaneously, thus minimizing the possibility of source confusion.

\subsubsection{Instrument Fundamentals}

Instruments used to detect $x$-rays are capable of measuring the time of arrival and the energy of a photon entering the detector. Some of these instruments (pixelated proportional chamber. silicon strip detector) also return the location of the photon hit on the detector. No information is available as to the direction the photon was traveling when it impacted the detector. But in order to use these photons to specify a direction to a target source. it is necessary to measure the direction a photon is traveling as it impacts the detector.

One way to determine the direction a photon is traveling is to place a collimator in front of the detector to limit its field of view. Any photon recorded by the detector then came from a small section of the sky defined by the collimator. In its simplest form a collimator can be a tube with walls dense enough and thick enough to block the path of photons incident upon them. A detailed analysis of the performance of collimators is given in Appendix $\mathrm{D}$ and outlined below.

Consider the two dimensional collimator shown in Figure 3.5. An arriving x-ray photon is constrained to move in a plane and is incident on the detector surface at an 
angle $\alpha$. Before the photon reaches the detector, it must pass between the two collimator walls. The collimator shadows the detector, reducing its effective length. The effective length of the detector is reduced from $L_{0}$ to $L$ by this shadowing effect and by the fact that the $\mathrm{x}$-rays are incident on the detector at an angle.

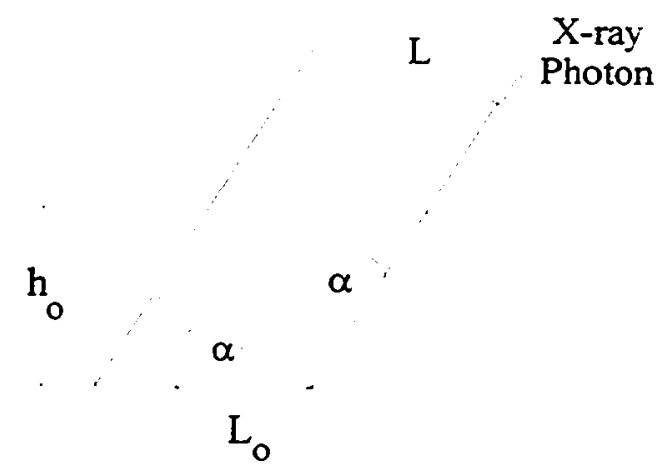

Figure 3.5: Two Dimensional Collimator Geometry
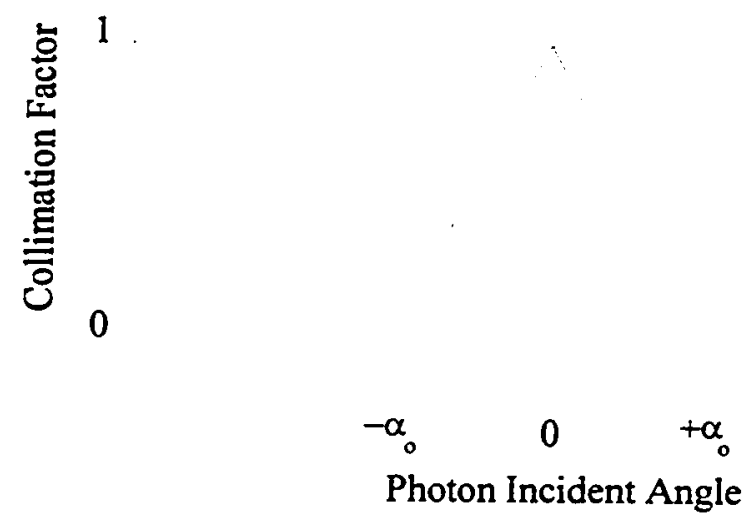

Figure 3.6: Ideal Two Dimensional Collimator Transmission 
The effectiveness of a two dimensional collimator can be described by a single parameter, $T_{c}$ that is a function of the incident angle. $\alpha$, and the instrument geometry:

$$
\begin{array}{ll}
\mathrm{T}_{c}=\left(1-\frac{\tan |\alpha|}{\tan \left(\alpha_{o}\right)}\right) \cos (\alpha), & |\alpha| \leq \alpha_{o} \\
\mathrm{~T}_{c}=0, & |\alpha| \geq \alpha_{o} \\
\text { where, } \tan \left(\alpha_{o}\right)=\frac{h_{o}}{L_{o}} &
\end{array}
$$

$\alpha_{o}$ is called the "full-width-half-max" of the detector and is also one half the instrument field-of-view.

For a collimator with a very narrow field-of-view (small $\alpha_{0}$ ), this parameter can be approximated by,

$$
\begin{array}{ll}
\mathrm{T}_{c}=\left(1-\frac{|\alpha|}{\alpha_{o}}\right), & |\alpha| \leq \alpha_{o} \\
\mathrm{~T}_{c}=0, & |\alpha| \geq \alpha_{o}
\end{array}
$$

This simplified expression is the equation for a triangle with base $2 \alpha_{0}$ and height 1 centered at $\alpha=0$. The collimator transmission. $T_{C}$ is shown as a function of the incident angle. $\alpha$ in Figure 3.6.

A simple three dimensional collimator can be constructed from one. or a series of. rectangular tubes. open on each end to allow for the passage of $x$-rays. The transmission of such a collimator can be modeled as if it is independent in two orthogonal directions. These orthogonal directions are called the principal directions of the collimator. The overall transmission is simply the product of the transmission of two two-dimensional collimators oriented orthogonally, viz.:

$$
T_{c}=\left(1-\frac{\tan |\rho|}{\tan \left(\rho_{0}\right)}\right) \cos (\rho)\left(1-\frac{\tan |\alpha|}{\tan \left(\alpha_{0}\right)}\right) \cos (\alpha)
$$


A contour plot of the transmission of such a collimator is shown in Figure 3.7. Note that the zero transmission lines (lines that define the instrument FOV) form a square. Lines of constant transmission are hyperbolae with asymptotes that are the zero transmission lines.

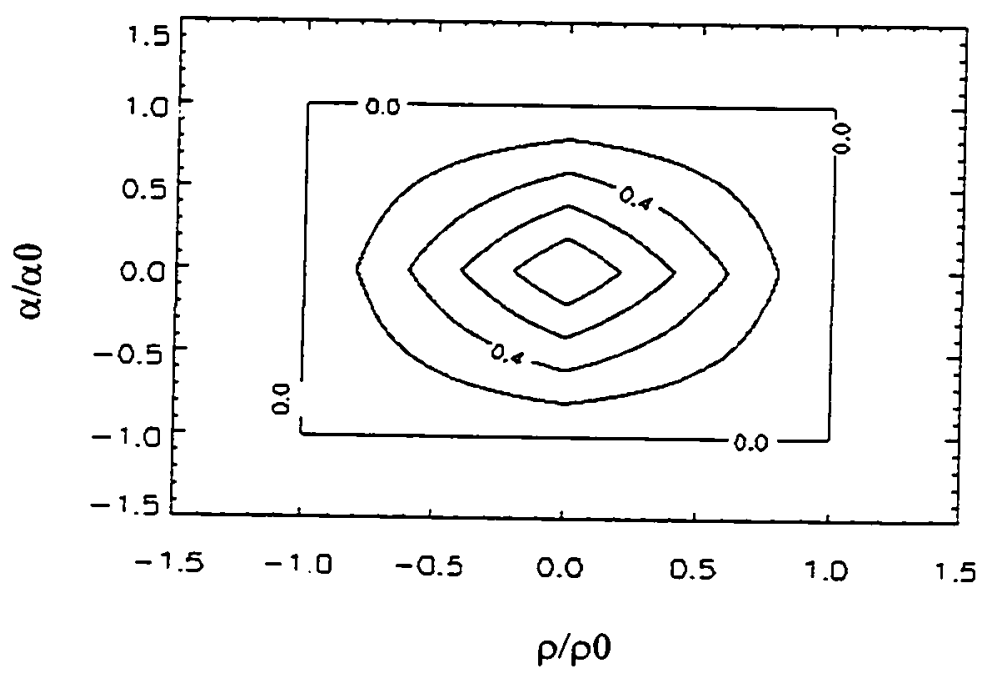

Figure 3.7: Ideal Three Dimensional Collimator Response

\subsubsection{General Star Scanner Description}

A series of simple $x$-ray star scanners can be constructed by combining a rectangular collimator with an $\mathrm{x}$-ray detector. The collimator must be oriented such that the detector line of sight is perpendicular to the spin axis and one of the detector principal axes is parallel to it. These directions must be known before the instrument is used as an attitude reference. As the spacecraft rotates, $x$-ray sources will come into the collimator field of view. A scan over the guide star is created by counting the photons and placing them in bins of constant elapsed time. If the spin rate and source intensity are constant during the scan. the number of counts recorded in each bin over a scan will map the 
triangular response of a two dimensional collimator. By matching the known transmission of the collimator to the shape of the scan. the orientation of the satellite can be determined.

In order to describe the operation of an x-ray star scanner it is necessary to describe the apparent motion of a guide star in the instrument frame of reference as a function of time. A complete derivation of the related equations of motion is included in Appendix E and summarized here. This model will be called the First Order Star Scanner Model.

First, several coordinate systems must be defined:

Celestial Coordinates - Inertially fixed frame. Denoted $\left(X_{c}, Y_{c} . Z_{c}\right)$. the $X_{c}$ axis points to galactic north while the $Y_{c}$ axis is in the direction of the first point of Aries.

Guide Star Coordinates - Inertially fixed frame. A guide star is defined by its position in celestial coordinates. This position is given by the right ascension of the ascending node (RA) and the declination (DEC) of the star. First. a rotation through $R A$ about the $X_{c}$ axis to a set of coordinates $\left(X^{\prime}, Y^{*} . Z^{*}\right)$ is performed. A rotation through DEC about the $Z^{*}$ axis to the guide star coordinates $(X, Y, Z$ ) is then made. This places the $Y$ axis pointing directly at the guide star. Derivation of all equations of motion will take place in the Guide Star inertial frame.

Spacecraft Coordinates - Body fixed frame. The spacecraft coordinates are in the principal directions of the inertia tensor. The $y$-axis points nominally out the instrument face. The z-axis is in the nominal spin direction. This system is defined by the unit vectors $(x, y, z)$. 
Instrument Coordinates - Body fixed frame. The $y_{i}$-axis is in the direction of maximum instrument transmission. The $x_{i}$-axis defines the pitch axis of the instrument. The $z_{i}$-axis defines the roll axis of the instrument. This coordinate system can be defined by in terms of the Spacecraft Coordinates by three euler rotations $\left(\delta_{x}, \delta_{y}, \delta_{z}\right)$.

The attitude motion of the spacecraft can be divided into two categories: torque free motion and forced motion. The forced motion is governed by the Euler Equations:

$$
\begin{aligned}
& T_{x}=I_{x x} \dot{\omega}_{x}+\left(I_{z z}-I_{y y}\right) \omega_{y} \omega_{z} \\
& T_{y}=I_{y y} \dot{\omega}_{y}+\left(I_{x x}-I_{z z}\right) \omega_{x} \omega_{z} \\
& T_{z}=I_{z z} \dot{\omega}_{z}+\left(I_{y y}-I_{x x}\right) \omega_{y} \omega_{x}
\end{aligned}
$$

These equations are written in the body fixed spacecraft coordinate system. The torque free or polhode motion of the vehicle can be described by:

$$
\begin{aligned}
& 0=I_{x x} \dot{\omega}_{x}+\left(I_{z z}-I_{y y}\right) \omega_{y} \omega_{z} \\
& 0=I_{y y} \dot{\omega}_{y}+\left(I_{x x}-I_{z z}\right) \omega_{x} \omega_{z} \\
& 0=I_{z z} \dot{\omega}_{z}+\left(I_{y y}-I_{x x}\right) \omega_{y} \omega_{x}
\end{aligned}
$$

While spin stabilized spacecraft are designed to have an inertially stable spin axis, there will always be some polhode motion and some forced motion present. As described in Appendix E, this motion causes the guide star to travel a complex path in instrument coordinates. Fortunately, if we assume that the period of the polhode motion is long when compared to the spin period of the satellite and that the spin axis is always close to the desired spin axis and that the misalignment of the instrument relative to the spacecraft is small, it can be shown that the guide star will track a simple path over the instrument. 
The collimator parameters, $\rho$ and $\alpha$, can be written in terms of the motion of the spacecraft. viz.:

$$
\begin{aligned}
& \rho=-\left(\Omega t+\delta_{z}\right) \\
& \alpha=-\left(\theta(0)+\delta_{x}\right)
\end{aligned}
$$

Note that during a scan the pitch angle to the guide star (described by $\alpha$ ) is a constant and that the roll angle (described by $\rho$ ) is linear with time. The transmission of the collimator can then be written as a function of time. viz.:

$$
T_{c}(\alpha, \rho)=T_{\alpha}\left(-\theta(0)-\delta_{x}\right) T_{\rho}\left(-\Omega t-\delta_{z}\right)
$$

The rolling motion of the vehicle causes the scan to be triangular shaped while the constant pitch angle defines the height of the peak.

Given the basic configuration of a detector. rectangular collimator and some electronics, there are three basic types of $x$-ray attitude measurement instruments that can be defined:

1. X-ray spin rate sensor

2. Single collimator $x$-ray star scanner

3. Differential collimator $x$-ray star scanner 


\subsubsection{X-ray Spin Rate Sensor}

A simple $x$-ray spin rate sensor can be constructed by simply measuring the time it takes for a guide star to pass through the FOV of the collimator. The spin rate of the satellite is directly proportional to the length of time required for the source to pass through the FOV. That is, it is proportional to the width of the triangle base. This instrument consists of the following components:

1. A collimator to define the field-of-view of the instrument.

2. A detector to count the photons passing through the collimator.

3. A threshold to indicate the presence of a guide star in the FOV and trigger the signal processing software.

4. Signal processing circuitry to fit collimator functions to selected scans to determine the spin rate of the satellite.

This instrument offers the following advantages:

1. The number of celestial sources radiating in $x$-rays is large enough to provide a number of candidates $(-100)$ for making the measurement. but small enough to minimize the problem of source confusion (vis-à-vis optical observations).

2. Measuring spin rate does not require knowledge of which source is being observed or of the spin phase. This reduces the need for on-board computation.

3. Using a collimator and solid-state detector provides for a durable. yet easily manufactured instrument. No optics or precision machining are required.

4. To first order, the measurement of spin rate is immune to calibration errors in the alignment of the collimator and the measurement of the gain of the detector.

5. A single. small detector can be used to measure this quantity. 


\subsubsection{Single Collimator X-ray Star Scanner}

An x-ray star scanner uses all of the information present in a scan to determine the orientation of the spacecraft. It is apparent from equation 3.3.7 that there is sufficient information in a scan to determine the roll angle and pitch angle of the spacecraft in guide star coordinates. This is in addition to the roll rate measurement as described above. If the guide star associated with the scan can be identified, two of the three angles defining the orientation of the spacecraft relative to the celestial sphere can be determined. This can be done either from a priori information (e.g. a previous attitude fix) or by observing the temporal characteristics of the guide star as it passes through the FOV. For the purposes of this discussion, the process of identifying the guide star is assumed to be handled separately from the process of determining the orientation of the vehicle. It is apparent, however, that the operation of this instrument will require a limited catalog of guide stars and their celestial coordinates to be kept on-board. A simple drawing of this instrument is given in Figure 3.8a.

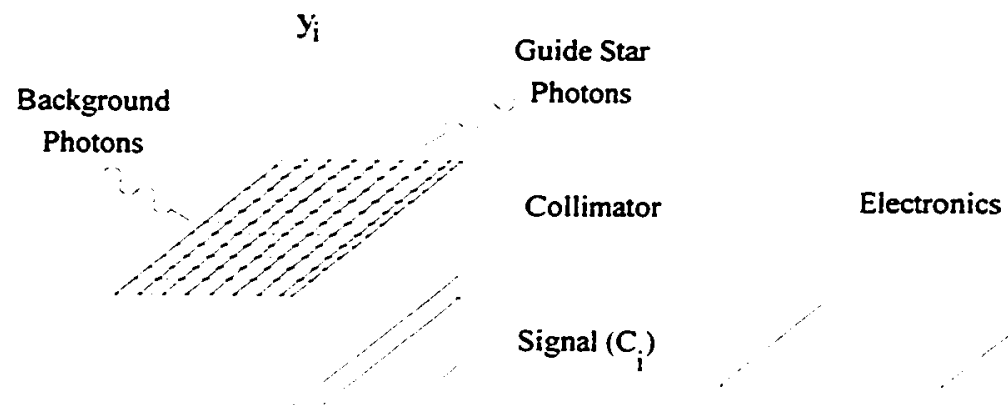

Detector

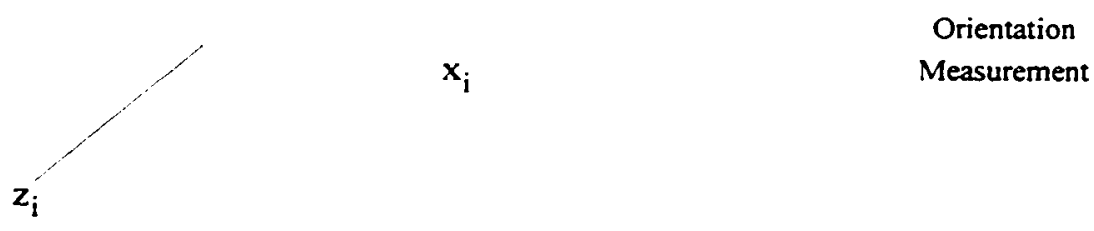

Figure 3.8a: Single Collimator X-ray Star Scanner Geometry 


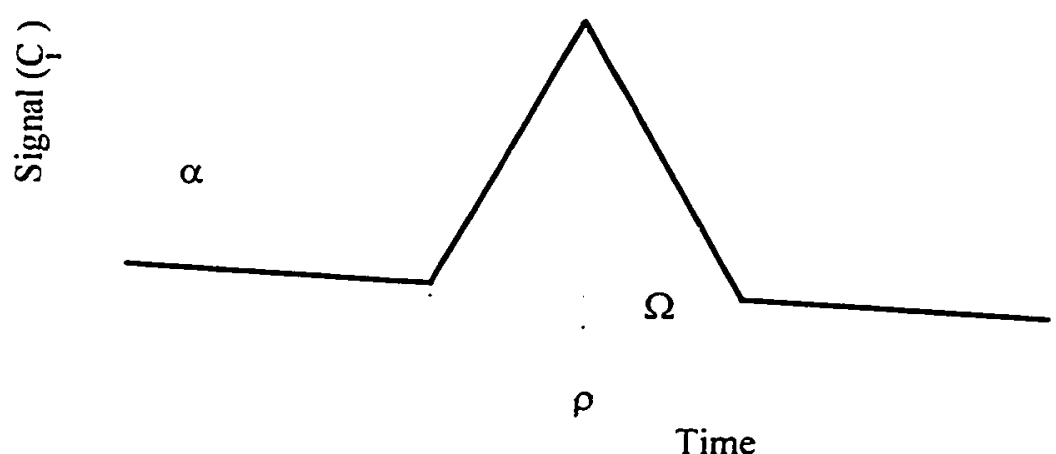

Figure 3.8b: Single Collimator X-ray Star Scanner Response

The measurement process can be described in terms of the number of photons present in a given bin, $Y_{i}$, during a scan, viz.:

$$
Y_{i}=A B_{i}+R A\left(1-\frac{\tan \left|\theta(0)+\delta_{x}\right|}{\tan \left(\alpha_{0}\right)}\right) \cos \left(\theta(0)+\delta_{x}\right)\left(1-\frac{\tan \left|\Omega_{t_{i}}+\delta_{z}\right|}{\tan \left(\rho_{0}\right)}\right) \cos \left(\Omega t+\delta_{z}\right)
$$

$$
\text { where, } \quad \begin{aligned}
& A=\text { Detector Area } \\
& B_{i}=\text { Background Count Rate } \\
& R=\text { Guide Star Intensity }
\end{aligned}
$$

Each scan over a known guide star will give two pointing measurements - the roll angle and the magnitude of the pitch angle (Figure 3.8b). At the time when the peak collimator transmission occurs, the line of sight to the guide star, the collimator normal and the spin axis all lie in the same plane. The sign of the pitch angle and the angle defining a rotation about the line of sight to the guide star are unobservable. By scanning over two sources successively the complete state of the satellite can be determined - with the exception of the pitch sign, which is still unobservable. The primary shortcoming of this instrument lies in its need for an a priori measurement of the guide star intensity. 
While a catalog of known intensities can be kept on board, a majority of the x-ray sources show significant temporal variability in their intensities. This variability will lead directly to errors in the pitch angle measurement.

\subsubsection{Differential Collimator X-ray Star Scanner}

The need for an a priori estimate of the guide star intensity presents a major limitation to the performance of an x-ray star scanner. By using two collimated detectors to produce a differential signal, it is possible to measure the "pitch" of the satellite spinaxis relative to a target source and do so independent of the source intensity. This is in addition to a measurement of the spin phase and rate. To produce the necessary differential pitch signal, the two detectors must be oriented such that a guide star passing into the field of view of the detectors appears at a different pitch angle to each. That is. the instrument coordinates of the two detectors must be offset in pitch $\left(\delta_{x}\right)$ relative to one another. The differential signal of the scans from each detector is a measure of the pitch angle and is independent of the instantaneous intensity of the guide star. The sign of the pitch angle becomes observable as well.

There are two basic design approaches to this type of $x$-ray star scanner. The first approach uses two completely independent instruments. each with its own collimator. detector and electronics (Figure 3.9a). The two independent signals then provide enough information to determine the spacecraft orientation as previously described (Figure $3.9 \mathrm{~b}$ ). Alternately, a single detector can be used with two independent collimators to provide the two signals (Figure $3.10 \mathrm{a}$ ). One collimator is canted in the pitch direction by $\div \delta_{\mathrm{v}}$ degrees. while the other collimator is canted by $-\delta_{x}$ degrees. In order to separate the two signals (one detector, two collimators) they must appear sequentially in time (Figure $3.10 \mathrm{~b})$. This can be done by canting the collimators apart in the roll direction as well $\left(\delta_{z}\right)$. If the collimators are not canted apart. the signal from the instrument will be the sum of the two signals rather than two peaks that can be fitted separately. 


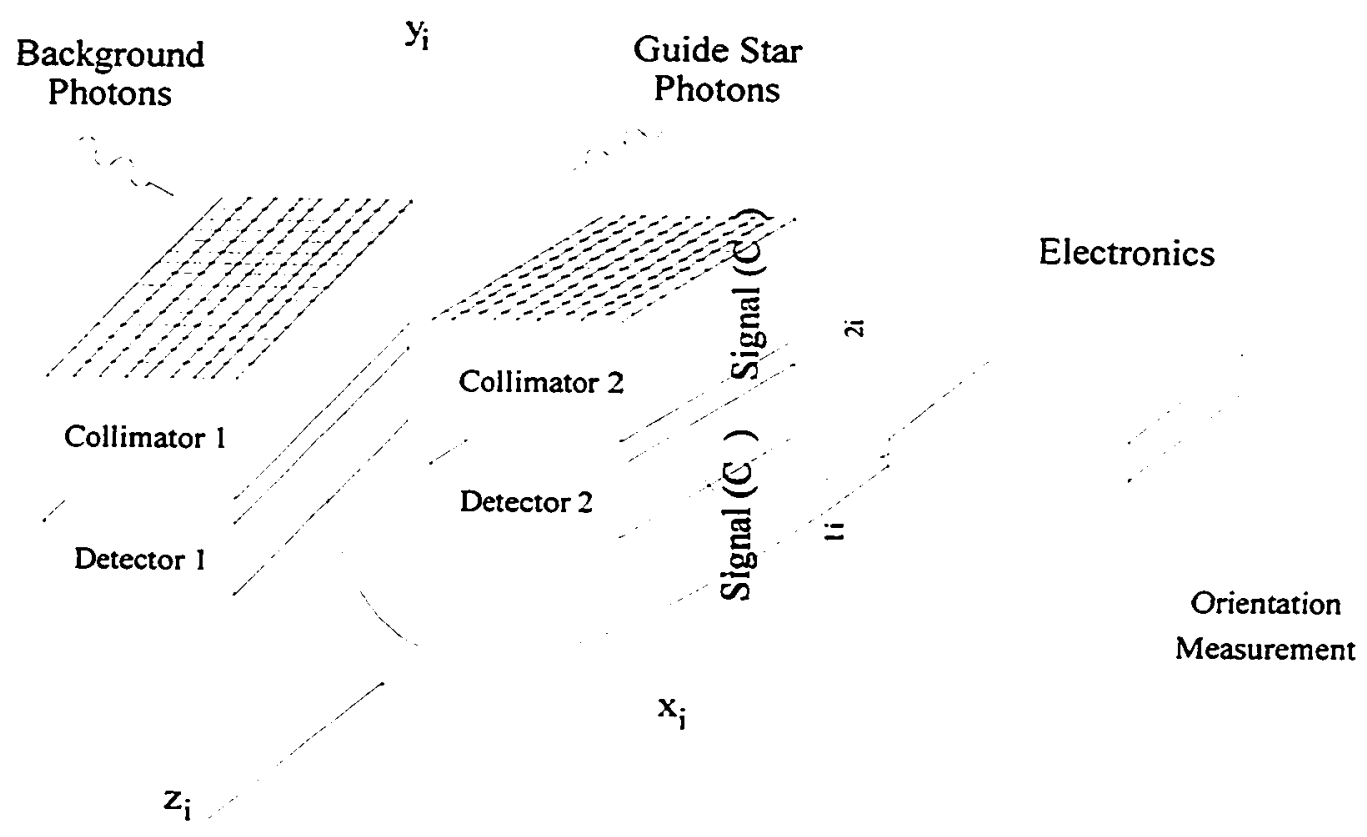

Figure 3.9a: Dual Collimator/Dual Detector X-ray Star Scanner Geometry
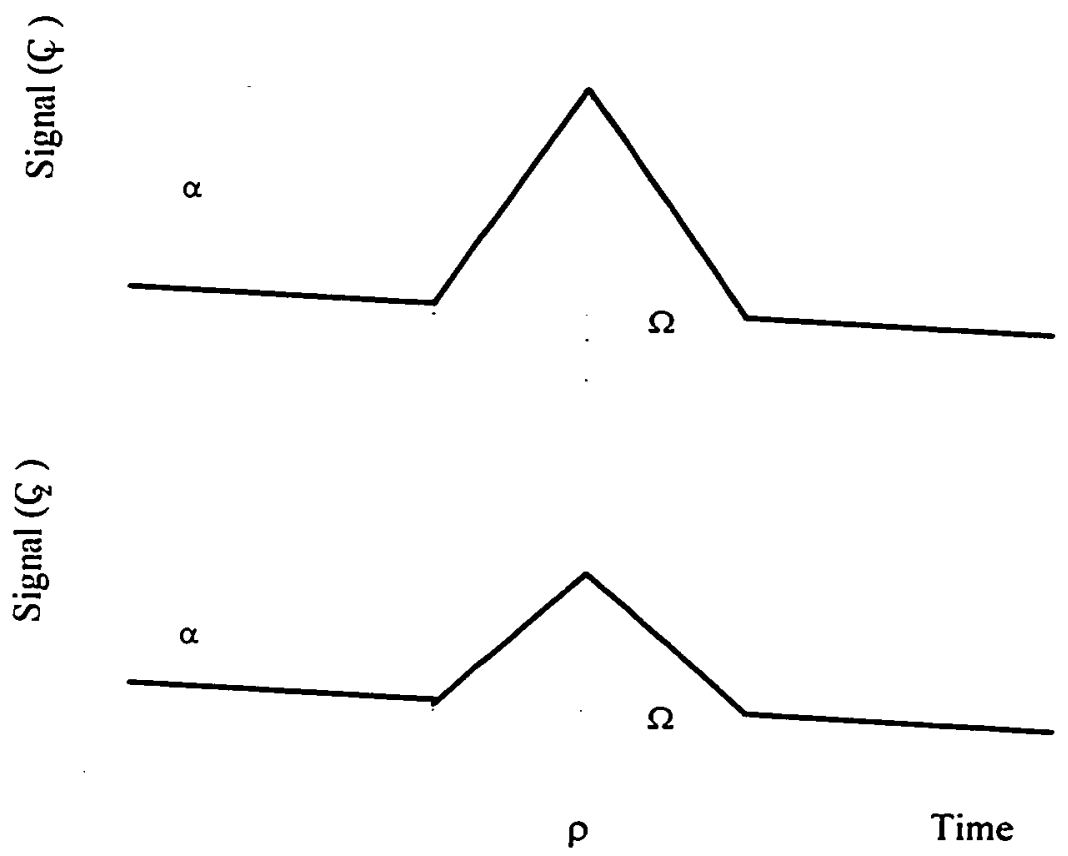

Figure 3.9b: Dual Collimator/Dual Detector X-ray Star Scanner Response 


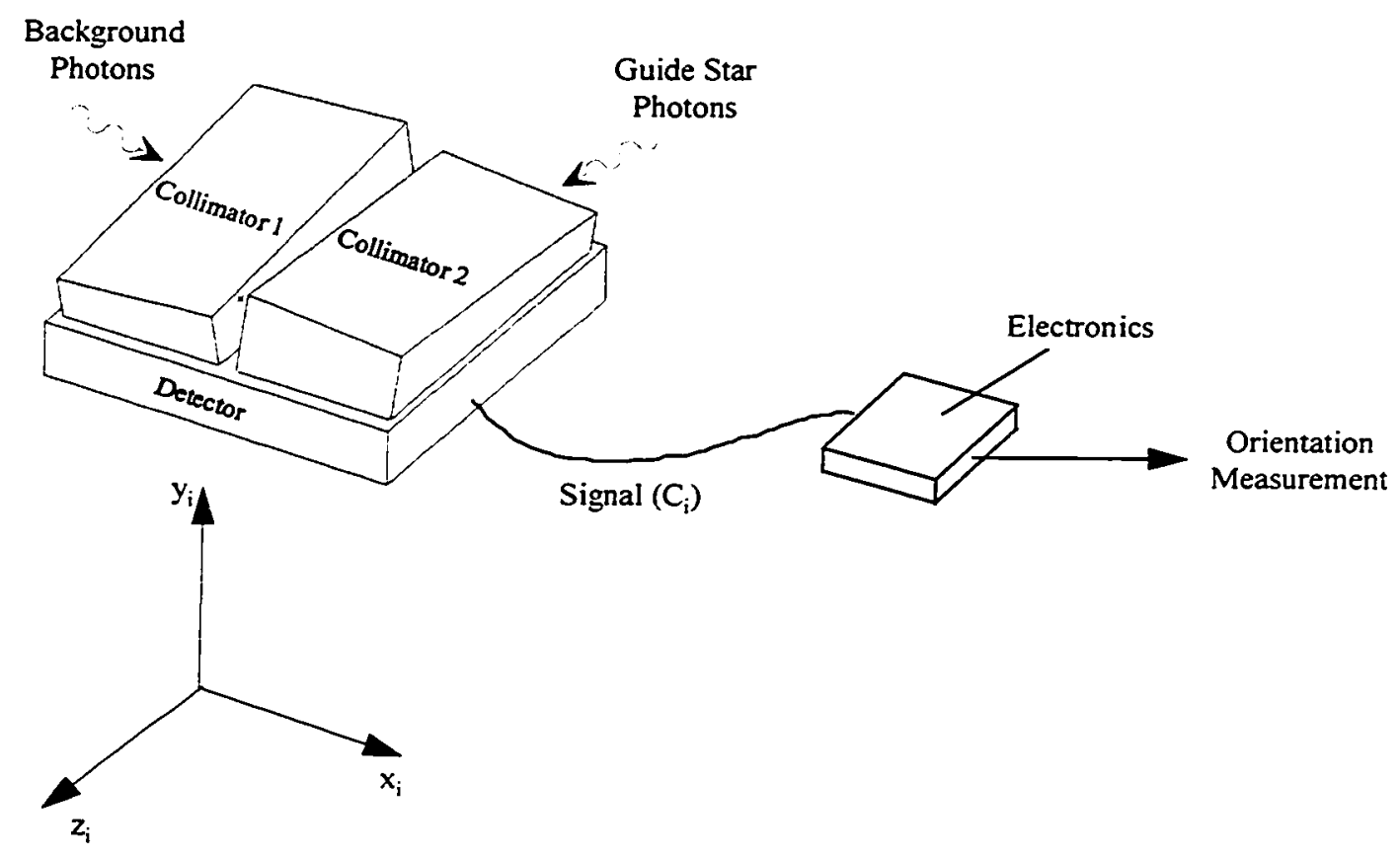

Figure 3.10a: Dual Collimator/Single Detector X-ray Star Scanner Geometry

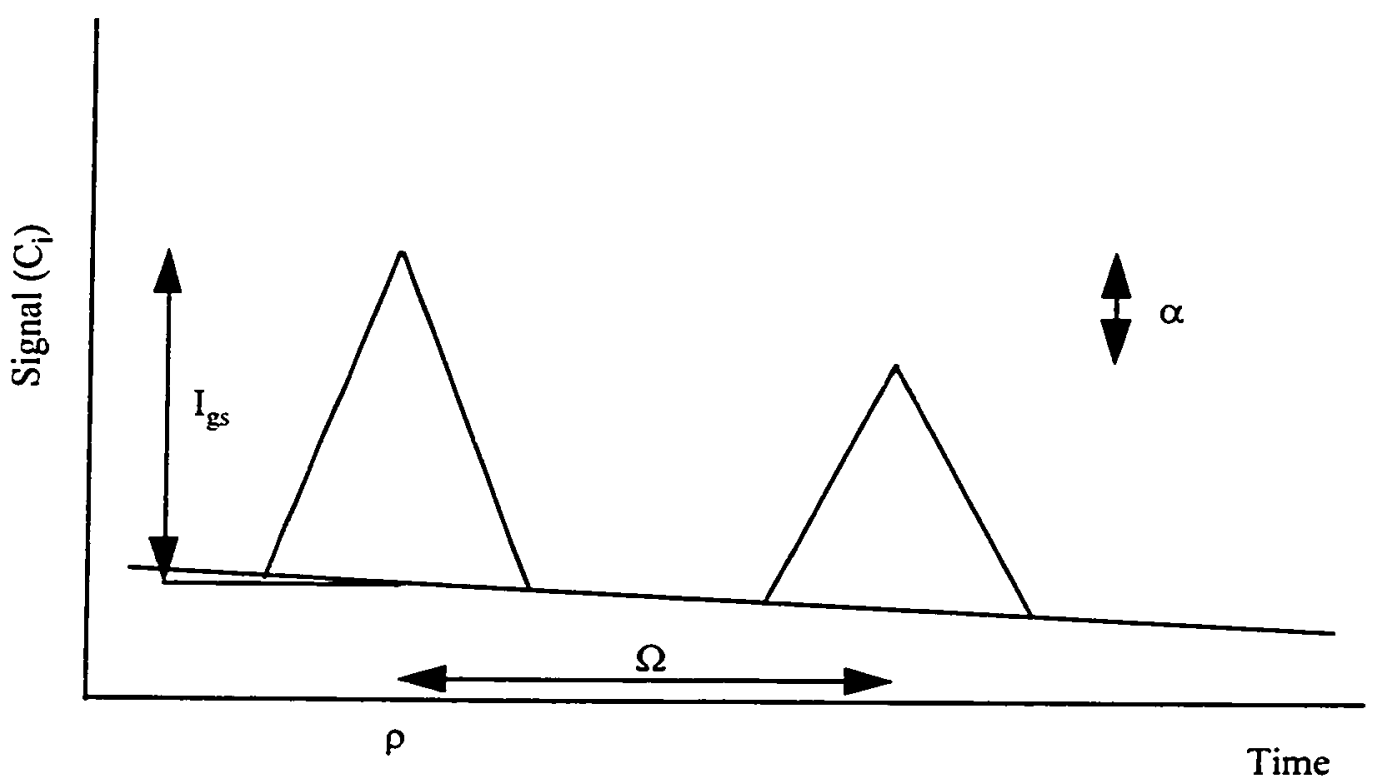

Figure 3.10b: Dual Collimator/Single Detector X-ray Star Scanner Response 
A comparison of the performance of a dual instrument versus a single instrument with dual collimators can be performed by examining the relative signal to noise ratios. A crude measure of SNR is the ratio of the average number of counts collected from the source divided by the standard deviation of the number of counts collected due to noise (Poisson fluctuations due to the source and background). The steady background can be removed during the fit routine.

By using two detectors, each with area A. the SNR for one of the detectors would be:

$$
\begin{aligned}
& \text { Signal }=S=A \frac{\rho_{o}}{\Omega} R \quad \rho_{o}=\text { FOV Half Cone Angle in Roll } \\
& \text { Noise }=N=\sqrt{A \frac{\rho_{o}}{\Omega}(B+R)} \quad \begin{aligned}
\mathrm{B} & =\text { Background Inten } \\
\text { where. } \mathrm{R} & =\text { Source Intensity }
\end{aligned} \\
& \begin{array}{ll}
S N R_{2}=\frac{S}{N}=\sqrt{A \frac{\rho_{o}}{\Omega}} \frac{R}{\sqrt{B+R}} & \\
& \Omega=\text { Roll Rate }
\end{array}
\end{aligned}
$$

An equivalent size single detector/dual collimator instrument has double the collecting area of the dual instrument model. But the background intensity is also doubled because there are two collimators feeding the detector. A "collimator packing factor", $(l \leq 1)$ is added to account for geometric constraints is placing the dual collimator system on the detector. This will reduce the effective area of the instrument.

$$
\begin{aligned}
& \text { Signal }=S=2 l A \frac{\rho_{o}}{\omega_{s}} R \\
& \text { Noise }=N=\sqrt{2 l A \frac{\rho_{o}}{\omega_{s}}(2 B+R)} \\
& S N R_{2}=\frac{S}{N}=\sqrt{2 l A \frac{\rho_{o}}{\omega_{s}}} \frac{R}{\sqrt{2 B+R}}
\end{aligned}
$$


By taking the ratio of the SNR's, the relative performance of these instruments as a function of the guide star and background intensities can be determined, viz:

$$
\frac{S N R_{1}}{S N R_{2}}=\frac{\sqrt{2 A l \rho_{o}} \frac{R}{\omega_{s}} \sqrt{2 B+R}}{\sqrt{A \begin{array}{l}
\rho_{o} \\
\omega_{s}
\end{array} \sqrt{B+R}}}=\sqrt{\begin{array}{r}
1+\frac{R}{B} \\
2 l \frac{B}{R} \\
B
\end{array}}
$$

It is apparent then that the single detector/dual collimator approach is superior to the dual detector approach when the ratio of the source strength to the background strength is greater than some function of the detector geometry, viz:

$$
\frac{R}{B} \geq \frac{2(1-l)}{2 l-1}
$$

In fact, if the collimator packing factor is unity, the single detector approach will always yield the superior instrument.

\subsubsection{Second Order Star Scanner Model}

The operation of an x-ray star scanner as manufactured will not be ideal. The peak of the collimator transmission will not be sharp as manufacturing errors will tend to round this peak off. Additionally. it will have tails where there is a measurable transmission at incident angles greater than the instrument field-of-view - a parameter that itself will not turn out to be exactly the desired value. Thus. it will be necessary to calibrate the collimators used on x-ray star trackers.

Once the collimator has been calibrated, there will be misalignments between the spin axis of the satellite and the orientation of the detector axes. These misalignments may be caused by integration errors in the placement of the collimator detector system or by a misalignment between the spacecraft coordinate system and the actual principal axes of the spacecraft. The polhode motion of the vehicle will also introduce errors in the orientation measurement process. By expanding the First Order Star Scanner Model to include second order small terms we arrive at The Second Order Star Scanner Model. Since this model includes the polhode motion of the vehicle and collimator misalignment 
terms, it should be possible to use it to improve the estimate of the instrument attitude from the scanned data. The derivation of this model is presented in detail in Appendix $F$ and summarized here.

To derive the second order model, we follow the derivation of our first order model. neglecting terms that are third order small and smaller. One additional assumption must be made. The spacecraft is assumed to be a body of revolution about the spin axis. The resulting description of the polhode motion in terms of the spacecraft angular velocity in body axes is:

$$
\begin{aligned}
& \omega_{x}=\dot{\omega}_{1}(0) t+\omega_{1}(0) \\
& \omega_{y}=\dot{\omega}_{2}(0) t+\omega_{2}(0) \\
& \omega_{z}=\Omega
\end{aligned}
$$

With the polhode motion $\left(\omega_{x}, \omega_{y}\right)$ decoupled from the spin motion $\left(\omega_{z}\right)$, these equations can be integrated in terms of the spacecraft euler angles to get the time history. of the spacecraft attitude, viz.:

$$
\begin{aligned}
& \phi=\Omega t \\
& \theta=\theta(0)+\theta(0) t \\
& \psi=\psi(0)+\dot{\psi}(0) t-\Omega \theta(0) t
\end{aligned}
$$

The location of the guide star in instrument coordinates can then be determined by transforming the guide star vector $\left(\bar{R}_{s}\right)$ first into spacecraft coordinates and then into instrument coordinates. The transformation from spacecraft coordinates into instrument coordinates accounts for the small. unknown misalignments in the detector orientation $\left(\varepsilon_{x}, \varepsilon_{y}, \varepsilon_{z}\right)$. The result is a time history of the guide star location in terms of the collimator angles. viz.:

$$
\begin{aligned}
& \rho=-\left(\Omega t+\delta_{z}+\varepsilon_{z}+\left(\theta(0)+\delta_{x}+\varepsilon_{x}\right)\left(\delta_{y}+\varepsilon_{y}\right)\right) \\
& \alpha=-\left(\theta(0)+\delta_{x}+\varepsilon_{x}\right)-\theta(0) t+\left(\varepsilon_{y}+\delta_{y}\right)\left(\Omega t+\delta_{z}+\varepsilon_{z}\right)
\end{aligned}
$$


There are three possible manufacturing errors in the placement of the collimator:

1. Rotation about spin axis $\left(\varepsilon_{z}\right)$

2. Rotation in pitch direction $\left(\varepsilon_{\mathrm{x}}\right)$

3. Rotation about instrument line of sight $\left(\varepsilon_{y}\right)$

There is only one term due to the polhode motion in this model - the pitch rate.

$\theta(0)$. While the collimator orientation angles $\left(\delta_{x}, \delta_{y}, \delta_{z}\right)$ are known parameters. it should be possible to extract some of the misalignment terms and the polhode motion term from scanned data.

\subsubsection{Rotation about Spin Axis}

A misalignment of the collimator about the spin axis $\left(\varepsilon_{z}\right)$ will introduce a bias of $\varepsilon_{\mathrm{z}}$ in the roll phase measurement. There will be no change in the transmission function of the collimator other than to shift it in roll phase. This misalignment cannot be observed and thus cannot be removed from the measurements.

\subsubsection{Rotation in Pitch Direction}

A misalignment of the instrument in the pitch direction $\left(\varepsilon_{x}\right.$ about the instrument $x-$ axis) will introduce a bias in the measurement of the spacecraft pitch angle. $\theta(0)$ of $\varepsilon_{\mathrm{x}}$. Again. this misalignment is unobservable and cannot be removed from the measurements.

\subsubsection{Rotation about Instrument Line of Sight}

A misalignment of the instrument about the instrument line of sight $\left(\varepsilon_{y}\right)$ will introduce some nonlinearities into the star scanner operations. First. the location of the peak transmission will be shifted by an amount $\theta(0) \varepsilon_{\mathrm{y}}$ in roll. It will also affect the shape of the collimator response function. The presence of this misalignment will cause the transmission of the collimator to be increased/decreased as the peak transmission is 


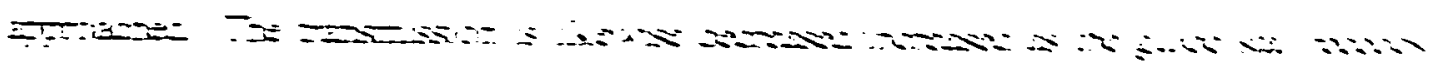
न

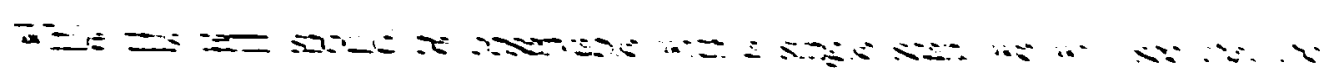

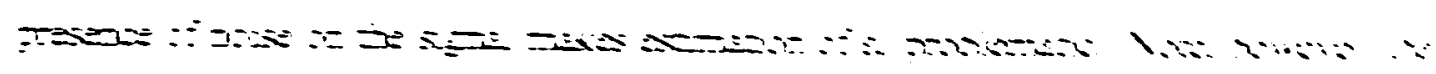

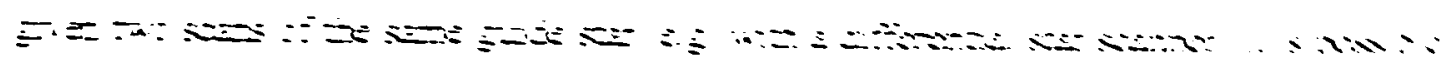

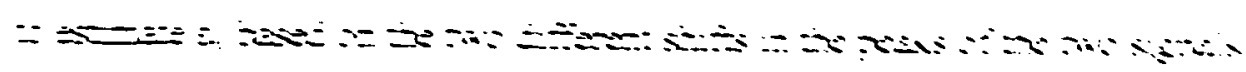

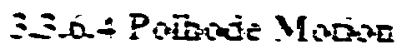

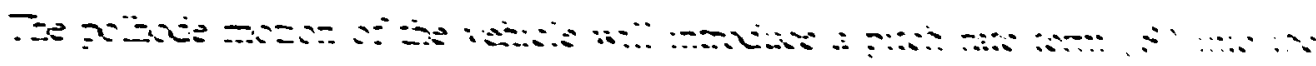

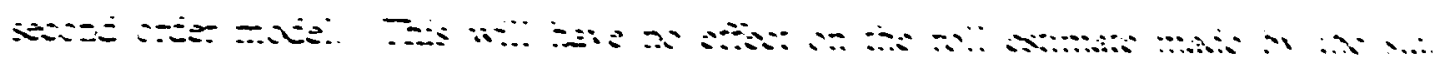

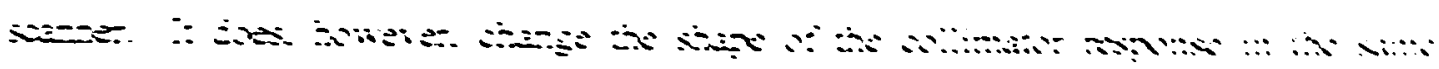

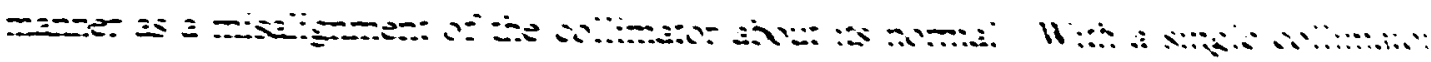

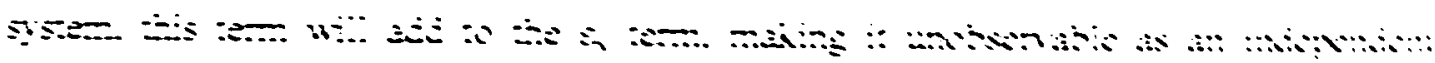

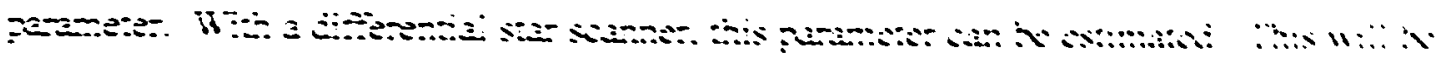

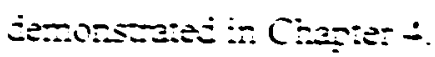




\section{Chapter 4: HEAO-A1 Attitude Determination Studies}

\subsection{Introduction}

The data from the HEAO-Al surveys of the $x$-ray sky provide an excellent opportunity to prove the concept of x-ray attitude determination on spinning spacecraft. The scanning data can be used to demonstrate the performance of both the single detector attitude determination scheme and the differential detector scheme.

\subsection{HEAO-A1 Description}

As previously described. the HEAO-1 mission (Figure 4.1) contained four experiments, one of which was the Large Area Sky Survey (LASS or HEAO-A1) consisting of seven proportional chambers sensitive to $x$-rays in the $250 \mathrm{eV}$ to $25 \mathrm{keV}$ range (Wood et al, 1984). All of the detectors were proportional chambers of the same basic design. although the collecting areas and collimators were different on modules 5 . 6 , and 7. The counting modules had three layers of high voltage wires that provided 

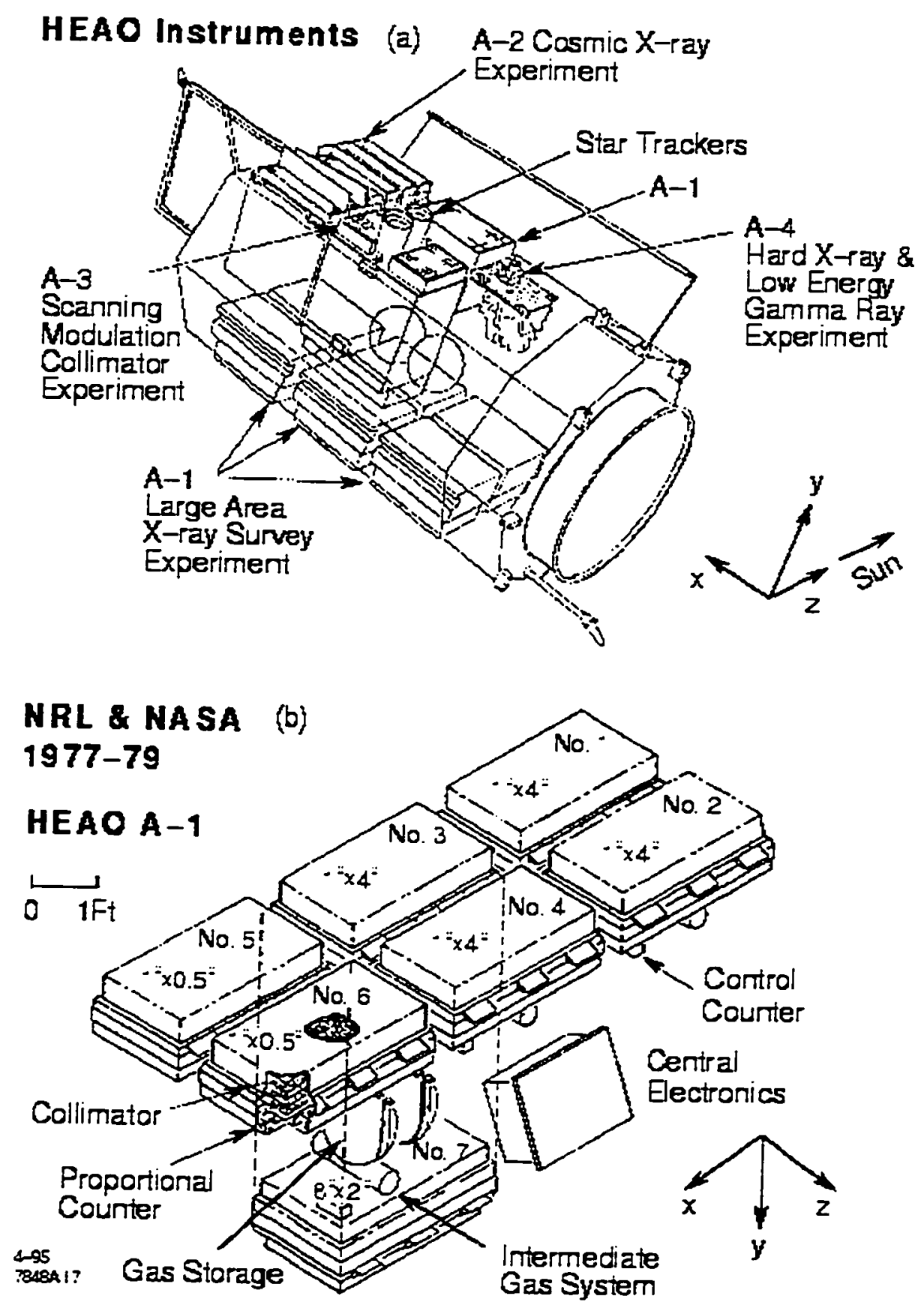

Figure 4.1: HEAO-Al LASS Mission 
counting as well as anticoincidence protection against charged particle events. These detectors operated with a counter gas that was $22 \%$ methane and $78 \%$ xenon at a nominal pressure of 2 psia. The gas was contained within the counter by the counter body on five sides (opaque to x-rays) and a $2.5 \mu \mathrm{m}$ Mylar film that was transparent to x-rays above $250 \mathrm{eV}$ on the sixth side. The window was supported against the gas pressure by a rectangular cell honeycomb stainless steel strongback with a stainless steel mesh between the two. The honeycomb strongback also formed the second stage of the collimator. A multigrid collimator was placed above the strongback to provide the first stage of collimation. These were a series of etched molybdenum sheets with spacer frames between them. A $2 \mu \mathrm{m} \mathrm{Kimfol} \mathrm{polycarbonate} \mathrm{film} \mathrm{with} \mathrm{a} \mathrm{second} \mathrm{surface} \mathrm{aluminum}$ reflector was placed above the multigrid collimator to act as a heat shield (Wood et al. 1984). The physical properties of each module are summarized in Table 4.1.

\begin{tabular}{|l|c|l|l|l|}
\hline $\begin{array}{c}\text { Sensor } \\
\text { Module }\end{array}$ & $\begin{array}{c}\text { FOV } \\
\text { (roll } \times \text { pitch) }\end{array}$ & \multicolumn{1}{|c|}{$\begin{array}{c}\text { View } \\
\text { Direction }\end{array}$} & $\begin{array}{c}\text { Collecting } \\
\text { Area }\left(\mathrm{cm}^{2}, \mathrm{~m}^{2}\right)\end{array}$ & \multicolumn{1}{|c|}{ Comments } \\
\hline 1 & $2 \mathrm{deg} \times 8 \mathrm{deg}$ & $-\mathrm{Y}$ & $1650(0.165)$ & Failed 9/23/77 \\
\hline 2 & $2 \mathrm{deg} \times 8 \mathrm{deg}$ & $-\mathrm{Y}$ & $1650(0.165)$ & Failed 9/22/77 \\
\hline 3 & $2 \operatorname{deg} \times 8 \mathrm{deg}$ & $-\mathrm{Y}$ & $1650(0.165)$ & Single Detector Case \\
\hline 4 & $2 \operatorname{deg} \times 8 \mathrm{deg}$ & $-\mathrm{Y}$ & $1650(0.165)$ & Failed 1/26/78 \\
\hline 5 & $2 \operatorname{deg} \times 1 \mathrm{deg}$ & $-\mathrm{Y}+1 / 3 \operatorname{deg} \mathrm{Z}$ & $1350(0.135)$ & Dual Detector Case \\
\hline 6 & $2 \operatorname{deg} \times 1 \mathrm{deg}$ & $-\mathrm{Y}-1 / 3 \operatorname{deg} \mathrm{Z}$ & $1350(0.135)$ & \\
\hline 7 & $16 \operatorname{deg} \times 4 \operatorname{deg}$ & $+\mathrm{Y}$ & $1900(0.135)$ & \\
\hline
\end{tabular}

Table 4.1: HEAO-A1 Module Properties

The transmission of the rectangular collimators of the HEAO-Al modules can be modeled as the product of two two-dimensional collimators acting in orthogonal directions as described in Chapter 3. These are the in-scan (roll) direction and the spin axis (pitch) direction. This model was validated for the HEAO mission by a Monte Carlo simulation before launch and by scans through the Crab nebula once the satellite was on orbit and operational (Ibid). These scans were also used to calibrate the collimator 
responses of the detectors for the purpose of generating the all-sky catalog. These calibrations were not used in the HEAO attitude determination studies described here.

All seven detectors were oriented nominally perpendicular to the spin axis of the satellite such that the spinning action caused them to sweep out great circles of the sky normal to the earth-sun line. Thus, every six months the entire celestial sphere would be scanned. The spin axis, called the ' $Z$-axis', was pointed at the sun and the nominal spin period was $0.00272 \mathrm{rad} / \mathrm{sec}$. After some initial processing by the detector electronics. the photons from these scans were sent to the A-1 central electronics module where they were binned in either $0.320 \mathrm{sec}(320 \mathrm{~ms})$ or $0.005 \mathrm{sec}(5 \mathrm{~ms})$ time bins depending on the mode of operation. These data are stored along with energy information. housekeeping information and the orientation of the spacecraft on a tape recorder prior to downlink to a ground station (Ibid).

Of critical importance to the HEAO-1 mission and particularly to this research was the presence of a precision attitude determination system on board. This system consisted of two BBRC CT-401 fixed-head star trackers from Ball Research and four single degree of freedom gyroscopes. Course and fine sun sensors were also present on the spacecraft for initial acquisition and station keeping (Fallon. Wertz et al 1990). The CT-401 star tracker has an $8 \mathrm{deg} \times 8 \mathrm{deg}$ field of view and has an accuracy of $+1-3$ arcminutes without calibration and $+/-10$ arcseconds when calibrated for such effects as temperature and star intensity variations. The calibration constants associated with these corrections are obtained from pre-flight testing (Ibid).

Information from these sensors was used to construct an aspect solution (as a function of time) for the spacecraft that was downlinked with the science data. This information was used in both the construction of the x-ray catalog and as a truth measurement in the attitude determination studies of this chapter. 


\subsection{HEAO-A1 Module 3 Attitude Determination Algorithm}

The scanning data from Module 3 (Mod3) of the HEAO-Al mission were used to demonstrate the concept of a single collimator x-ray star scanner. Furthermore, a sufficient number of trial scans existed to predict the performance of such an instrument. This analysis was done using the Interactive Data Language (IDL) on a UNIX workstation.

The steps taken to determine the attitude of the HEAO spacecraft from Mod3 scanned data are:

1. Select a single scan of the guide star. A sample of such a scan is shown later in Figure 4.2.

2. Fit an appropriate response function to the scan.

3. Determine the location of the guide star in detector coordinates from the fitted parameters.

4. Compare this to the known location of the guide star as given by the spacecraft aspect solution.

5. Generate an error signal in detector coordinates based on this difference.

The database from the HEAO-Al mission is maintained by the Particle Astrophysics Group at SLAC by Professor Lynn Cominsky and graduate students Han Wen and Andrew Lee. This consists of scanning data from Modules 3 and 5 (some of which is binned at $0.320 \mathrm{sec}$ and some of which is binned at $0.005 \mathrm{sec}$ ), pointed data from the high bit rate mode operations and programs necessary to process this information. The data from a series of scans by Module 3 over the Crab nebula were used in this study. Module 3 data were selected for two reasons: Module 3 was operational and stable for most of the mission and its large field-of-view in the pitch direction allowed it to see a given guide star for a number of scans. While the database consists of scans over hundreds of different sources, proving the star scanner concept only requires the analysis 
of the performance of the instrument in scanning a single guide star. The Crab nebula was chosen as the guide star for several reasons:

1. Being the fourth brightest $\mathrm{X}$-ray source in the sky (after the sun, Sco-X1 and $\mathrm{GX} 4+1$ ), it offers an excellent SNR at "large" instrument pitch angles.

2. The Crab is the "standard candle" of the $\mathrm{x}$-ray sky. Problems due to the temporal variability of $\mathrm{x}$-ray sources are minimized.

3. There are no bright sources in the vicinity of the Crab. Source confusion is eliminated.

4. The Crab Nebula has a pulsar at its center with a pulse period of 0.033 $\sec (33 \mathrm{~ms})$. This offers a tie-in to $\mathrm{x}$-ray timing studies discussed in Chapter 5.

5. The scanning data from the Crab have been used extensively by Han Wen in various other work. This made them readily available in a usable form.

The scans of Module 3 over the Crab were selected from the larger database by software written by Dr. Ken Fairfield. This data subset consists of the scanned data binned at $0.320 \mathrm{sec}$ and the satellite aspect solutions at each time bin from the spacecraft attitude determination system. Each scan is constructed by concatenating three to five successive major frames. For the $320 \mathrm{msec}$ data, a major frame is divided into 128 bins. each $0.320 \mathrm{sec}$ long. The number of photons recorded by the detector are integrated over successive $320 \mathrm{msec}$ segments and recorded in each bin. Each major frame is $40.96 \mathrm{sec}$ long. Each scan is between $122 \mathrm{sec}$ and $205 \mathrm{sec}$ in length and contains a total of 384 to 640 bins.

When a subset of the Module 3 data is requested, that subset actually includes scans where the guide star was just out of the field of view of the instrument. This ensures that every usable scan is retrieved. Each of these must then be checked visually to eliminate those where the source does not appear or where there was an error in the detector. As an example, there were two scans where the data ended before the guide star 
had passed through the collimator field of view completely. All sets where the data showed a complete scan over the guide star were kept in the subset. Some of the scans contained bins where the number of photons counted in that time step were in error. For example, the background rates (i.e. bins where the guide star was not in the field of view) were generally 20 to 50 counts per bin. In a few cases a bin that should have contained only background photons had a value of 2000 counts or more, while the bins preceding and following it had values corresponding to the expected background rate. The fitting algorithm employed to estimate the spacecraft orientation was specifically designed to be immune to such effects. This algorithm will be discussed in detail later.

The detectors used on HEAO-A1 had a deadtime associated with their processing electronics. Deadtime is the length of time it takes a detector to process a given photon hit during which the detector is insensitive to further x-rays. If a photon arrives at the detector before it has finished process the previous photon hit, the newly arriving photon will not be registered. The net effect of deadtime is to underestimate the number of photons arriving in a given bin. The data used here were corrected for the deadtime effect per the work of Han Wen in AstroGravity Note \#14 (Han Wen. 1994) with an estimated detector deadtime of $1.4 \times 10^{-5} \mathrm{sec}(14 \mu \mathrm{sec})$. This correction is,

$$
\begin{aligned}
& C_{3}=\frac{C_{3 d}}{1-C_{3 d} T_{d}} T_{b} \\
& C_{3}=\text { Counts Corrected for Deadtime Effects } \\
& \mathrm{C}_{3 \mathrm{~d}}=\text { Counts from Database } \\
& \mathrm{T}_{\mathrm{d}}=\text { Detector Deadtime } \\
& \mathrm{T}_{\mathrm{b}}=\text { Binning Time }(320 \mathrm{msec})
\end{aligned}
$$

A representative scan of the Crab, after it has been corrected for deadtime effects is shown in Figure 4.2. The bins were numbered according to the IDL standard for arrays from 0 to $\mathrm{N}-1$ where $\mathrm{N}$ is the number of bins in a given scan. 


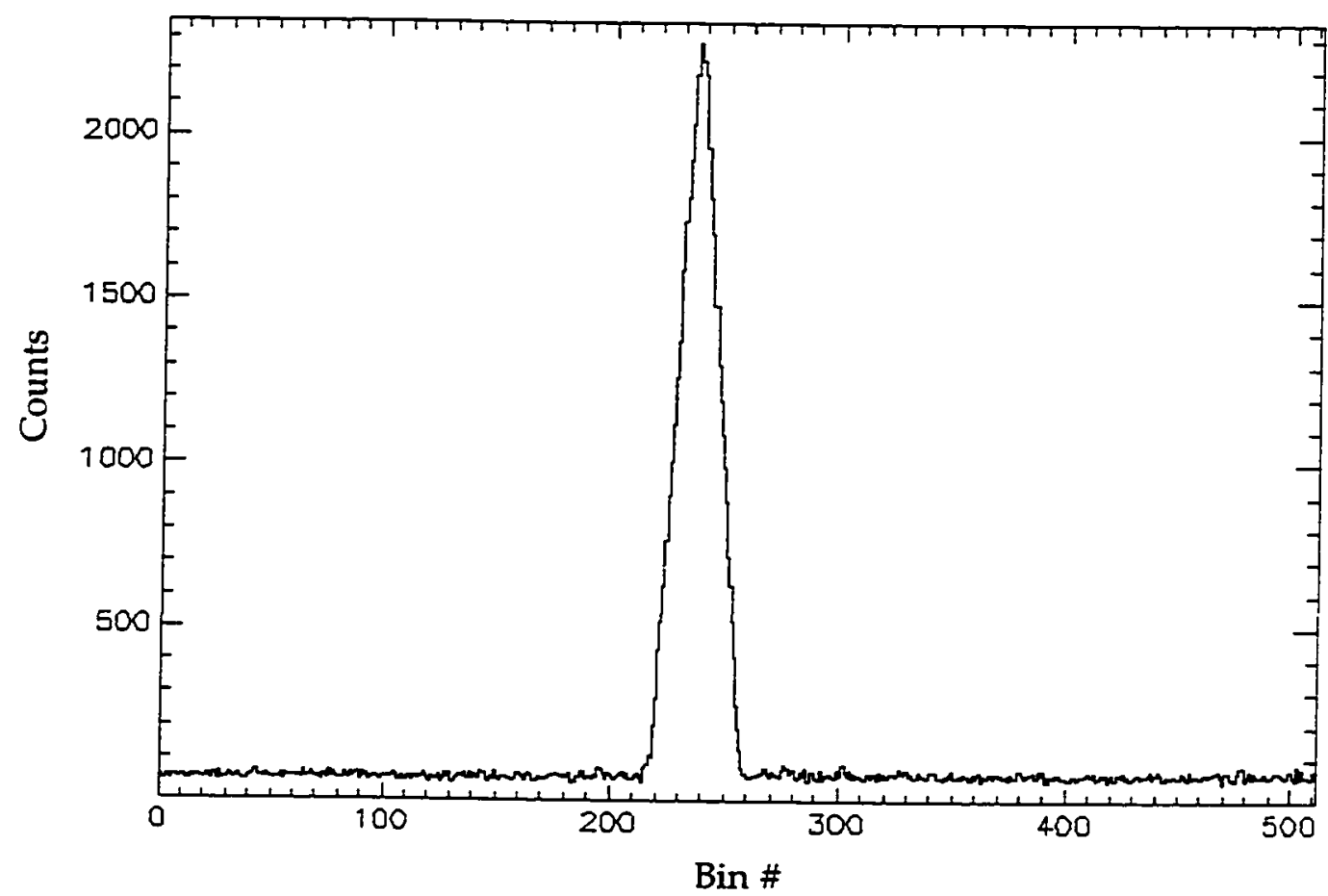

Figure 4.2: Sample HEAO-A1 Module 3 Data

The scan shown in Figure 4.2 is the sum of those photons collected by scanning over the guide star (the peak) and background photons (the constant offset), viz.:

$$
\begin{aligned}
Y_{i}=\left(B_{i}+R T_{i}\right) A \\
\mathrm{Y}_{\mathrm{i}}=\text { Counts in Bin } \mathrm{i} \\
\mathrm{B}_{\mathrm{i}}=\text { Background Rate in Bin } \mathrm{i} \\
\text { where, } \mathrm{R}=\text { Source Rate } \\
\mathrm{T}_{\mathrm{i}}=\text { Collimator Transmission at Bin } \mathrm{i} \\
\mathrm{A}=\text { Geometric Area of Detector }
\end{aligned}
$$


The background can be modeled by a simple function that is linear with time. The function has two parameters. $B_{0}$ and $B_{f}$, representing the number of background counts in the first and last bins of the scan respectively, viz.:

$$
B_{i}=B_{0}+\frac{i\left(B_{f}-B_{0}\right)}{N-1}
$$

where, $\begin{aligned} & \mathrm{B}_{0}=\text { Background Rate at } \mathrm{Bin} 0 \\ & \mathrm{~B}_{\mathrm{f}}=\text { Background Rate at Bin } \mathrm{N}-1\end{aligned}$

The guide star intensity, $\mathrm{R}$, must be given by a priori information. In this case. the intensity of the Crab Nebula is 4.121 counts $/\left(\mathrm{sec}^{*} \mathrm{~cm}^{2}\right)\left(4.121\left(10^{4}\right)\right.$ counts $\left./\left(\mathrm{sec}^{*} \mathrm{~m}^{2}\right)\right)$ (Wood et al. 1984).

Recalling from Chapter 3 and Appendix D that the transmission of a rectangular collimator can be represented by the product of two independent, orthogonal one dimensional collimators, viz:

$$
T_{i}=T_{\alpha i} T_{\rho i} \quad \text { where. } \begin{aligned}
& T_{\alpha i}=\text { Transmission in Pitch at Bin i } \\
& T_{\rho i}=\text { Transmission in Roll at Bin i }
\end{aligned}
$$

The individual transmission functions are.

$$
\begin{array}{ll}
\mathrm{T}_{\alpha i}=\left(1-\frac{\tan \left|\theta(0)+\delta_{x}\right|}{\tan \left(\alpha_{o}\right)}\right) \cos \left(\theta(0)+\delta_{x}\right), & \left|\theta(0)+\delta_{x}\right| \leq \alpha_{o} \\
\mathrm{~T}_{\alpha i}=0, & \left|\theta(0)+\delta_{x}\right| \geq \alpha_{o} \\
\mathrm{~T}_{\rho i}=\left(1-\frac{\tan \left|\Omega t_{i}+\delta_{z}\right|}{\tan \left(\rho_{o}\right)}\right) \cos \left(\Omega t_{i}+\delta_{z}\right), & \left|\Omega t_{i}+\delta_{z}\right| \leq \rho_{o} \\
\mathrm{~T}_{\rho i}=0 . & \left|\Omega t_{i}+\delta_{z}\right| \geq \rho_{o}
\end{array}
$$


Recalling that $t=0$ is defined to be the time when the instrument roll angle. $\rho$. is zero, $t_{i}$ can be written as follows:

$$
t_{i}=t_{b}\left(i-i_{p}\right) \quad \text { where. } \quad \begin{aligned}
t_{h} & =\text { Bin Size }(\mathrm{sec}) \\
i_{p}= & \text { Bin Number Where } \\
& \text { Roll Angle is Zero }
\end{aligned}
$$

Combining equations 4.3.2 through 4.3.6, the signal from the x-ray star scanner. $\mathrm{Y}_{3 \mathrm{i}}$ can be written in terms of the fit parameters. viz.:

$$
Y_{t}=\left(B_{i}\left(B_{0}, B_{f}\right)+R T_{i}\left(i_{p}, \theta(0), \Omega\right)\right) A
$$

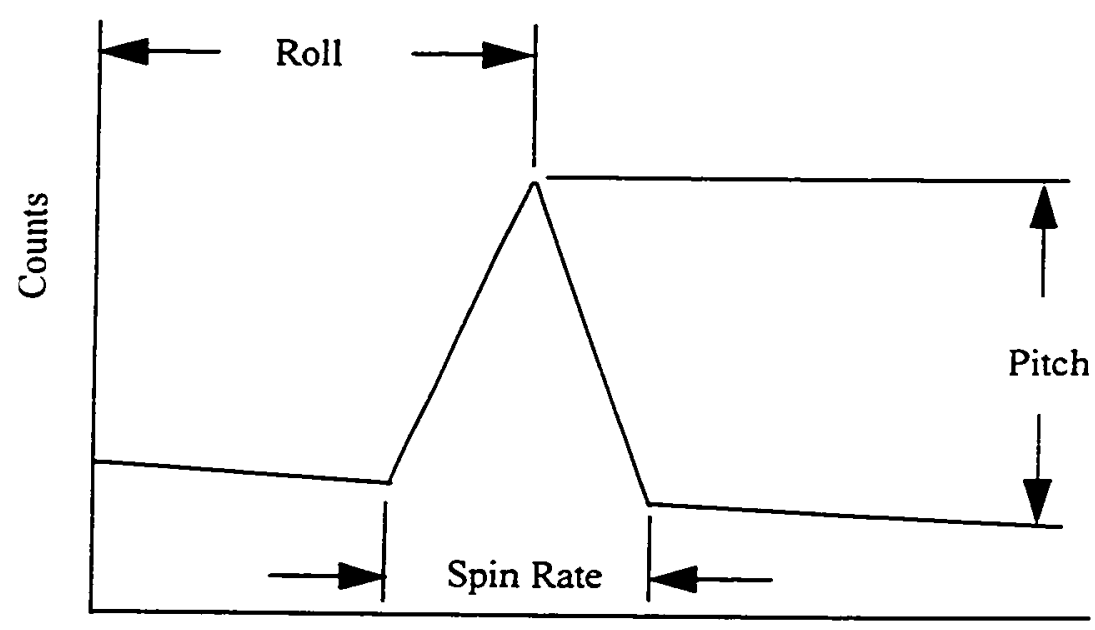

Time

Figure 4.3: Single Detector Fit Parameters 
Summarizing the five parameters in the model that we will fit (Figure 4.j):

1. The location of the peak transmission is a measure of the roll phase of the vehicle $\left(i_{p}\right)$.

2. The height of the triangle is a measure of the pitch angle of the spin axis $(\theta(0))$.

3. The width of the triangle is a measure of the spin rate of the vehicle $(\Omega)$.

$4 \& 5$. The background is modeled as a linear function of time $\left(\mathrm{B}_{0} \cdot \mathrm{B}_{\mathrm{f}}\right)$.

The parameter vector is $P=\left[B_{0}, B_{f}, i_{p}, \Omega, \theta(0)\right]^{\tilde{3}}$

The number of counts actually measured by the HEAO-A1 Module 3 detector in bin $i$ of a scan is $C_{3 i}$. The problem is to find a set of parameters that makes $Y_{3 i}$ the most probable representation of the data. $\mathrm{C}_{3 i}$.

As described in Section 3.2.2. the number of photons arriving at the detector in a given time bin can be modeled by a Poisson distribution. That is. the probability of detecting $\mathrm{n}$ photons is governed by,

$$
P(X=n)=e^{-\mu-A t_{b}} \frac{\left(\mu A t_{b}\right)^{n}}{n !} \text { where. } \mu=\text { Total Intensity }
$$

As the mean value of the distribution. $\mu \mathrm{At}_{\mathrm{b}}$. becomes large. the Poisson distribution approaches the Gaussian distriburion. viz:

$$
\lim _{\mu A t_{b} \rightarrow \infty} P(x \leq n)=\int_{-\infty}^{n} \frac{1}{\sqrt{2 \pi \mu A t_{b}}} e^{-\left(z_{\left.-\mu A t_{b}\right)^{2}}^{\mu A t_{b}}\right.} d z
$$


The number of counts in a given bin can be represented by a Gaussian distribution with mean. $\mu A t_{b}$ and standard deviation. $\sqrt{\mu A t_{b}}$.

The set of parameters, $P$, that provide the maximum likelihood solution to a data set with additive Gaussian noise is that set minimizing the Chi-squared error (Bevington. Robinson, 1992), viz.:

$$
\min \chi^{2}=\sum_{i=0}^{N-1} \frac{\left[Y_{3 i}(P)-C_{3 i}\right]^{2}}{\sqrt{C_{3 i}}}
$$

This cost function was minimized by using a gradient search algorithm supplied by the IDL software called "CRVFIT". This method requires the user to supply the derivatives of the fitted curve. $Y_{3 i}$, taken with respect to the fit parameters. The fitted curve used here is discontinuous at the edges of the field-of-view and at the peak of the transmission. Therefore, the derivatives of $Y_{3 i}$ were computed numerically.

To start the gradient search it is necessary to make an initial guess of the solution vector (the fitted parameters. P). It is not necessary to be extremely accurate in the initial guess. However. since the model of the data is nonlinear, the search can converge to different solutions given widely varying initial guesses. An easily computed starting point for the two background parameters $\left(B_{o}, B_{f}\right)$ is a constant at the level of the average of the number of counts per bin over the whole scan. viz.:

$$
B_{0}=B_{f}=\frac{1}{N} \sum_{j=0}^{N-1} C_{3 j}
$$

The nominal spin rate for the HEAO satellite is $0.00272 \mathrm{rad} / \mathrm{sec}$. This is used as the initial guess for the spin rate parameter, $\Omega$. Since no information about the pitch angle. $\theta(0)$, is available, $\theta(0)$ was initially set to zero. The zero roll bin. $i_{p}$. is the most critical initial guess in guaranteeing that the fitting process converges to the correct solution. This is due to the presence of random data upsets (discussed previously). For example. a simple criterion for selecting an initial value for $i_{p}$ is to set it equal to the bin containing the most counts - expecting this bin to be near the middle of the peak in the scan. If there is an upset in the data that causes a background bin to have more colints than any bin in 
the peak, the fit process will not converge properly. This can be avoided by using a slightly more complicated criterion: The number of counts in each bin are replaced by the average number of counts in that bin, the preceding bin and the following bin. The initial guess for the zero roll bin, $i_{p}$, is set to the bin with the most total counts, viz:

$$
f\left(i_{p}\right)=\max _{i}\left(\sum_{j=i-1}^{i+1} C_{3 j}\right)
$$

The result of this fitting process is a measure of the satellite orientation with the maximum likelihood of representing the true set of parameters that created the scan. This process was used to estimate the orientation of the satellite from 99 independent scans of the Crab Nebula.

Each estimate of the satellite orientation was then compared to the truth measure of the spacecraft orientation to determine the errors in the star scanner estimates. The truth measurements were provided by the on-board star trackers and were recorded four times per major frame. The actual spacecraft orientation at each time bin was determined by linearly interpolating between the two nearest truth measurements. These measurements provided the pointing directions of the spacecraft $y$ and $z$ axes in celestial coordinates.

Given the position of the guide star in celestial coordinates (from the HEAO Catalog) and the orientation of the spacecraft in celestial coordinates. the spacecraft orientation can be transformed into guide star coordinates per Appendix E. The location of the guide star can then be represented by the instrument pitch and roll angles, $\rho_{i}$ and $\alpha_{i}$ as a function of the time bin. $i$. The $x$-ray star scanner estimate of the spacecraft orientation occurs at just one time bin. $i_{p}$. At this point the estimated instrument roll angle is 0 , while the instrument pitch angle is $\theta(0)$. The true spacecraft roll rate can be determined from successive orientation solutions. viz.:

$$
\Omega_{t}=\sin ^{-1}\left(\frac{\hat{Y}\left(i_{b}\right) \cdot \hat{Y}\left(i_{b}+1\right)}{t_{b}}\right)
$$


The error in the star scanner orientation estimate can now be determined. viz.:

$$
\begin{aligned}
& \alpha_{e}=\left|\alpha_{t}\right|-|\theta(0)| \\
& \rho_{e}=\rho_{t}-0 \\
& \Omega_{e}=\left|\Omega_{t}\right|-|\Omega|
\end{aligned}
$$

Note that the pitch angle error and roll rate error involve the differences in the magnitudes of the truth measures and the star scanner estimates. This is due to the fact that the sign of the pitch angle and the sign of the roll rate are not observable by an x-ray star scanner. The results of this study are shown in Figure 4.4.
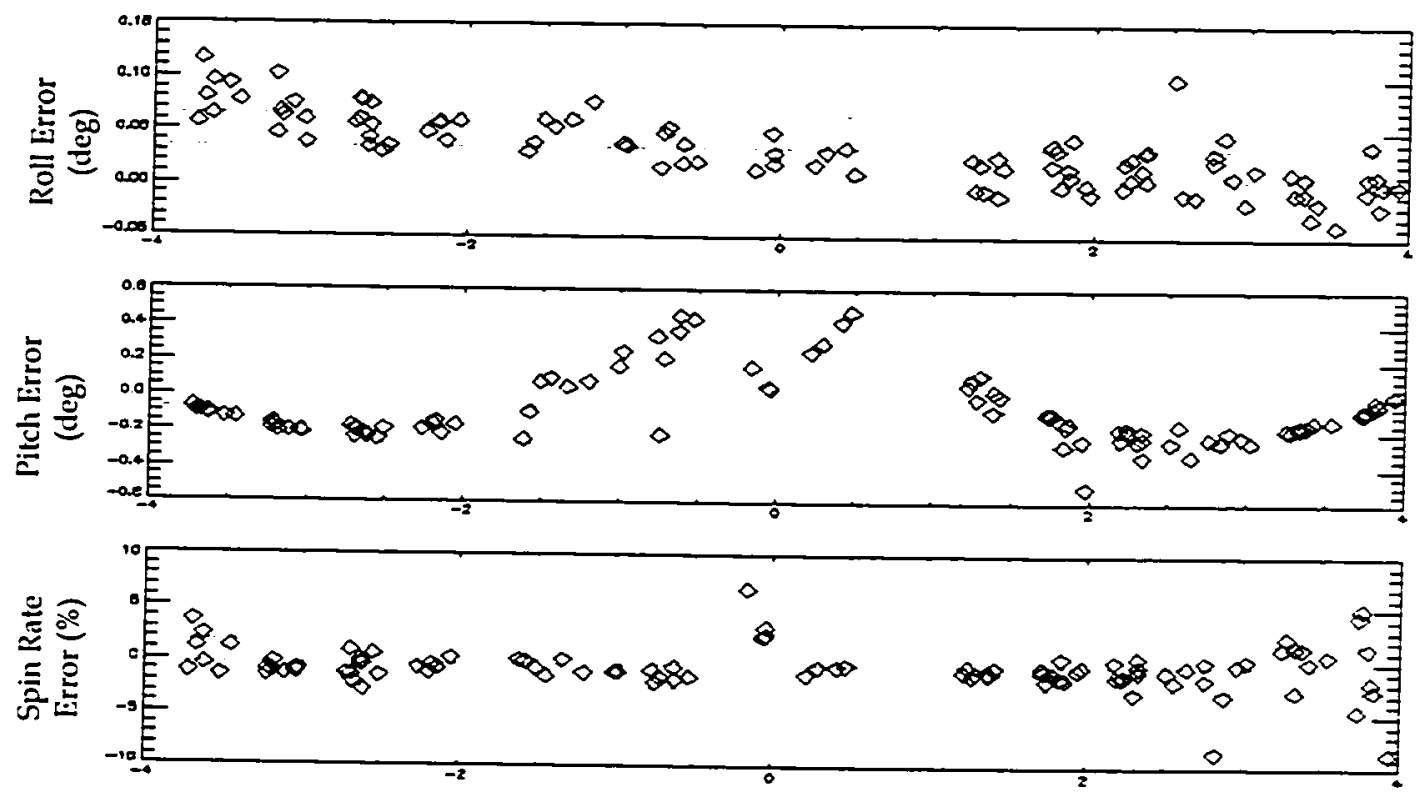

Pitch Angle (deg)

Figure 4.4: Single Detector Results

This study demonstrates the general concept of using an x-ray star scanner to determine the orientation of a spinning spacecraft. The error in the roll estimate over the 99 scans had a mean of $0.032 \mathrm{deg}$ and a standard deviation of $0.030 \mathrm{deg}$. When plotted as a function of the true pitch angle. the roll error appears to be a linear function of the pitch 
angle. This is consistent with a slight misalignment of the instrument axes relative to the spacecraft axes as discussed in Section 3.3.6.

The errors involved in measuring the pitch angle are much greater $(-0.084 \mathrm{deg}$ mean and $0.19 \mathrm{deg}$ standard deviation). The large errors in the pitch angle estimate can be attributed to two factors. First and foremost, the pitch angle estimate a function of the a priori estimate of the guide star intensity. This estimate may be in error either due to the inherent temporal variability of the guide star or due to errors in the intensity measurements reported in the HEAO Catalog. An underestimation of the guide star intensity will lead to an over estimate in the pitch angle that is proportional to the magnitude of the true pitch angle. That is, when the guide star is near the limit in the instrument field of view, the total guide star signal will be small and the error in the pitch angle estimate will be small. When the guide star is close to the instrument line of sight. the total guide star signal will be large and the error the pitch angle will be at its greatest. Note that the overall structure of the pitch error shown in Figure 4.4 is consistent with an underestimate in the intensity of the Crab Nebula.

The simple model of the collimator transmission used here may also lead to errors in the pitch estimate. The collimator transmission, as manufactured, will be more complicated than the simple linear model described in Chapter 3. Calibration of the $x$-ray star scanner before launch would allow a more accurate model of the instrument to be used.

The measurement of the spin rate has a bias of $-0.56 \%$ of the actual rate and a standard deviation of about $2 \%$. The bias may be introduced by differences between the approximate geometry of the collimator and its actual geometry. That is, if the actual field of view of the collimator is $1 \%$ larger than the anticipated field of view $\left(\rho_{0}\right)$, the spin rate estimate will be $1 \%$ slow. Note also that the spin rate estimates at the edges of the collimator field of view show decreasing accuracies. This is due to the fact that the smaller signal present at large pitch angles makes it more difficult to fit the collimator transmission to the data. 
Three of the roll rate estimates made when the pitch angle was nearly zero have errors as large as 7\%. This can be attributed to the error in the estimate of the guide star intensity. This intensity was probably underestimated as described above. At pitch angles near zero, the fitting process has a problem - it cannot make the fitted peak high enough to match the data. The closest fit to the data has zero pitch angle (to get as close as possible to the peak) and a wider base than the data. This wider base results in a spin rate estimate that is slower than the true spin rate.

\subsection{HEAO-A1 Module 3/5 Attitude Determination Algorithm}

There are two major drawbacks to using a single detector as an x-ray star scanner:

1. The sign of the pitch angle cannot be determined.

2. The magnitude of the pitch angle estimate is dependent on an a priori estimate of the intensity of the guide star. Any error in the estimate of the intensity leads to an error in the estimate of the pitch angle. The previous study with a single collimator instrument demonstrates this.

A differential x-ray star scanner can be constructed by using two single collimator $\mathrm{x}$-ray star scanners in conjunction as described in Chapter 3. If each scanner is oriented such that during a scan the guide star is at different pitch angles in each instrument fame. then the relative strengths of the two signals is a measure of the pitch angle of the spacecraft and is independent of the guide star intensity. In fact. one of the parameters in the model will be the instantaneous guide star intensity.

By using data from the Module 5 (Mod5) detector in conjunction with that from the Module 3 detector, the operation of a differential detector as an X-ray star scanner can be demonstrated. The Mod5 detector was offset by $1 / 3 \mathrm{deg}$ in pitch relative to the Mod3 detector which was aligned with the spacecraft axes. It also had a much smaller field-ofview in the pitch direction ( $0.5 \mathrm{deg}$ vs. $8 \mathrm{deg}$ for Mod3). Unfortunately, this difference in collimator geometry means that a double solution exists for the pitch angle measurement 
- one on either side of the Mod5 pointing direction. This ambiguity is shown in Figure 4.5. The section of the Module 5 pitch axis transmission curve that is non-zero falls completely within that portion of the Module 3 pitch axis transmission that is non-zero. Thus, there are two possible spacecraft pitch angles that could generate any single scan. Each solution will have a different measure of the instantaneous guide star intensity. Since we no longer use a priori information about this parameter, there is no way to determine which solution is correct. This was resolved by generating both solutions and selecting the solution with the proper pitch angle sign based on the sign of the truth measurement. Note that a dual colliınator instrument specifically designed as a star scanner would not have this ambiguity problem.

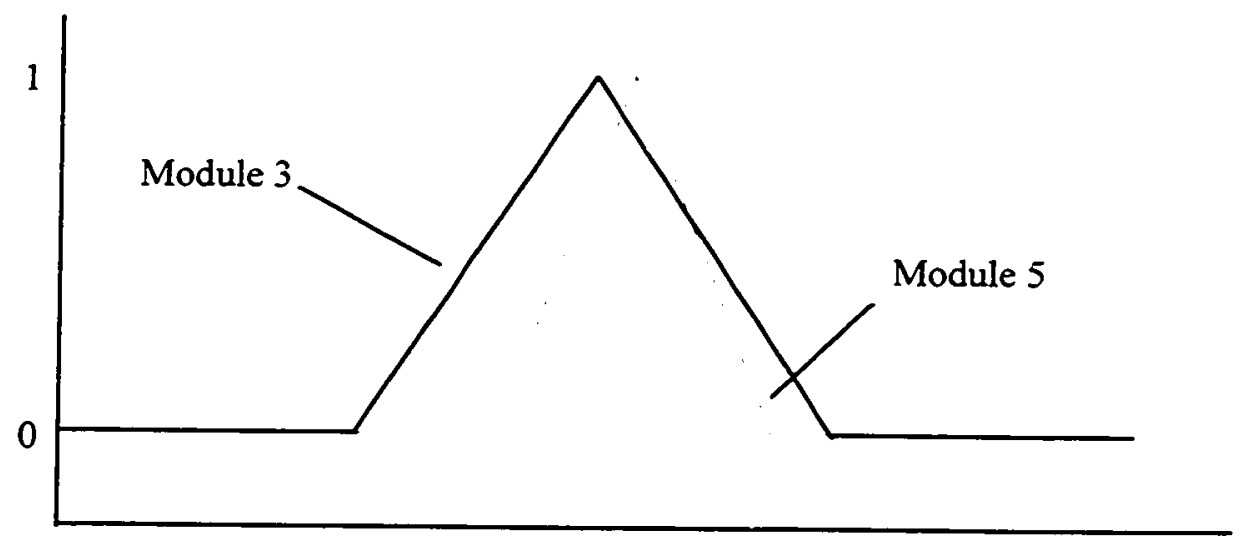

Time

Figure 4.4: HEAO-A1 Differential Detector Ambiguity 
The same basic algorithm was followed for the differential star scanner analysis as was followed for the single collimator star scanner analysis:

1. Select a single scan where the target source appears in both Module 3 and Module 5.

2. Fit an appropriate response function to the scans.

3. Determine the location of the source in instrument coordinates from the fitted parameters.

4. Compare this to the known location of the source as given by the spacecraft aspect solution.

5. Generate an error signal based on this difference.

In order to allow direct comparison to the results for the single detector, the Crab was chosen as the guide star for the dual detector analysis. Because the FOV of Module 5 is considerably smaller than that of Module 3, only a small subset of the scans available to Module 3 are also available to Module 5. This subset was further reduced by the fact that Module 5 was not operational at all times. The count rates for the data from both Module 3 and Module 5 were corrected for deadtime effects as described previously. The deadtime for Module 5 was assumed to be $14 \mu \mathrm{sec}$ since the detectors had identical electronics suites. An exampie of the data from the two modules corresponding to one scan is shown in Figure 4.6.

In addition to differences in the signals caused by the geometry of the two collimators. Module 3 and Module 5 have different collecting areas. These differences in geometry were accounted for in the modeling process. Thie modeling process for the differential star scanner case is similar to that for the single collimator star scanner case with three exceptions. The backgrounds for each detector were modeled as linear functions in time with independent parameters for each. The intensity of the guide star was added to the model. The roll phase, pitch angle and guide star intensity parameters make it necessary to perform the fit on the two scans simultaneously. 

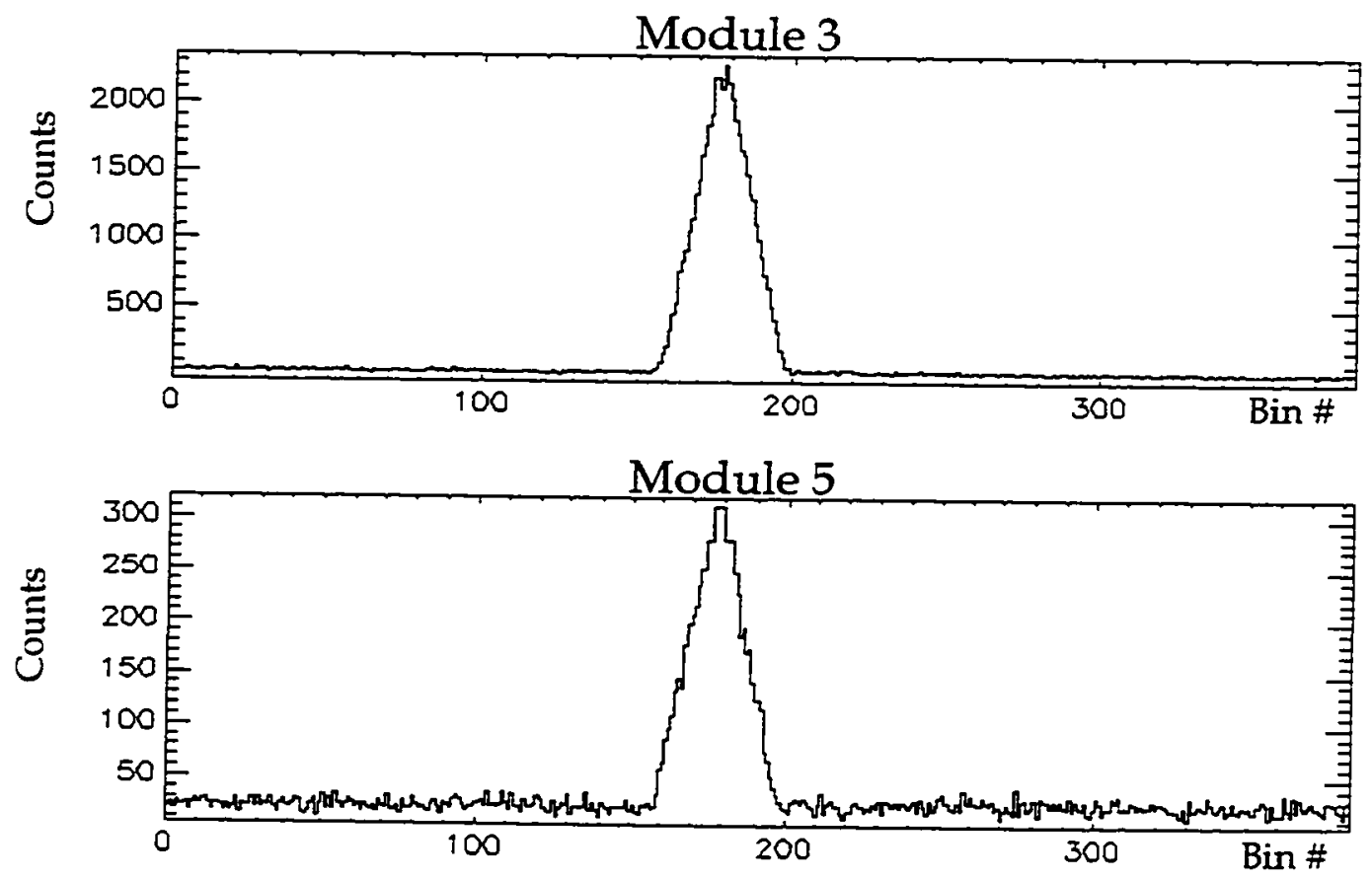

Figure 4.6: Dual Detector Sample Data

Reviewing Section 4.3. the number of counts in any bin of a scan by Module 3 or 5 can be written as,

$$
\begin{aligned}
& Y_{3 i}=\left(B_{3 i}+R T_{3 i}\right) A_{3} \\
& Y_{5 i}=\left(B_{5 i}+R T_{5 i}\right) A_{5}
\end{aligned}
$$

$Y_{\mathrm{ji}}=$ Counts in Bin $\mathrm{i}$ of Module $\mathrm{j}$

$\mathrm{B}_{\mathrm{ji}}=$ Background Rate in Bin $\mathrm{i}$ of Module $\mathrm{j}$

where. $\mathrm{R}=$ Source Rate

$\mathrm{T}_{\mathrm{ji}}=$ Module $\mathrm{j}$ Collimator Transmission at Bin $\mathrm{i}$

$A_{j}=$ Geometric Area of Module $j$ Detector 
The background seen by each detector is modeled as a linear function in time. viz.:

$$
\begin{aligned}
& B_{3 i}=B_{30}+\frac{i\left(B_{3 f}-B_{30}\right)}{N-1} \quad \text { where, } \begin{aligned}
& \mathrm{B}_{30}= \text { Background Rate at } \\
& \text { Bin } 0 \text { of Module } 3 \\
& \mathrm{~B}_{3 \mathrm{f}}= \text { Background Rate at } \\
& \text { Bin } N-1 \text { of Module } 3
\end{aligned} \\
& B_{5 i}=B_{50}+\frac{i\left(B_{5 f}-B_{50}\right)}{N-1} \quad \begin{aligned}
B_{50} & =\text { Background Rate at } \\
& \text { Bin } 0 \text { of Module } 5 \\
\mathrm{~B}_{5 f}= & \text { Background Rate at }
\end{aligned} \\
& \text { Bin N-1 of Module } 5
\end{aligned}
$$

The transmission of Module 3 is modeled as in Section 4.2. The Module 5 transmission is modeled similarly. The axes of the Module 5 collimator are not aligned with the spacecraft axes. This is modeled by the $\delta_{5 \mathrm{x}}$ term that is now non-zero.

$$
\begin{array}{ll}
\mathrm{T}_{5 \alpha i}=\left(1-\frac{\tan \left|\theta(0)+\delta_{5 x}\right|}{\tan \left(\alpha_{5 o}\right)}\right) \cos \left(\theta(0)+\delta_{5 x}\right), & \left|\theta(0)+\delta_{5 x}\right| \leq \alpha_{5 o} \\
\mathrm{~T}_{5 \alpha i}=0, & \left|\theta(0)+\delta_{5 x}\right| \geq \alpha_{5 o} \\
\mathrm{~T}_{5 \rho i}=\left(1-\frac{\tan \left|\Omega t_{i}+\delta_{5 z}\right|}{\tan \left(\rho_{5 o}\right)}\right) \cos \left(\Omega t_{i}+\delta_{5 z}\right), & \left|\Omega t_{i}+\delta_{5 z}\right| \leq \rho_{5 o} \\
\mathrm{~T}_{5 \rho i}=0 . & \left|\Omega t_{i}+\delta_{5 z}\right| \geq \rho_{5 o}
\end{array}
$$

Following the notation of Section 4.2. the signals from the differential star scanner can be modeled, viz.:

$$
\begin{aligned}
& Y_{3 i}=\left(B_{i}\left(B_{30}, B_{3 f}\right)+R T_{3 i}\left(i_{p}, \theta(0), \Omega\right)\right) A_{3} \\
& Y_{5 i}=\left(B_{i}\left(B_{50}, B_{5 f}\right)+R T_{5 i}\left(i_{p}, \theta(0), \Omega\right)\right) A_{5}
\end{aligned}
$$


Summarizing the eight parameters in the model that will be fitted to the data (Figure 4.7):

1. The location of the peak transmission is a measure of the roll phase of the vehicle $\left(i_{p}\right)$.

2. The difference in the heights of the triangles is a measure of the pitch angle of the spin axis $(\theta(0))$.

3. The absolute heights of the triangles is a measure of the intensity of the guide star (R).

4. The width of the triangles is a measure of the spin rate of the vehicle $\left(\omega_{\mathrm{s}}\right)$.

5-8. the background is modeled as a linear function of time. The background seen by each module is modeled independently $\left(B_{30}, B_{3 f}\right.$, $\left.\mathrm{B}_{50}, \mathrm{~B}_{5 f}\right)$.

The parameter vector is $P=\left[B_{30}, B_{3 f}, B_{50}, B_{5 f}, i_{p}, \Omega, \theta(0), R\right]^{T}$

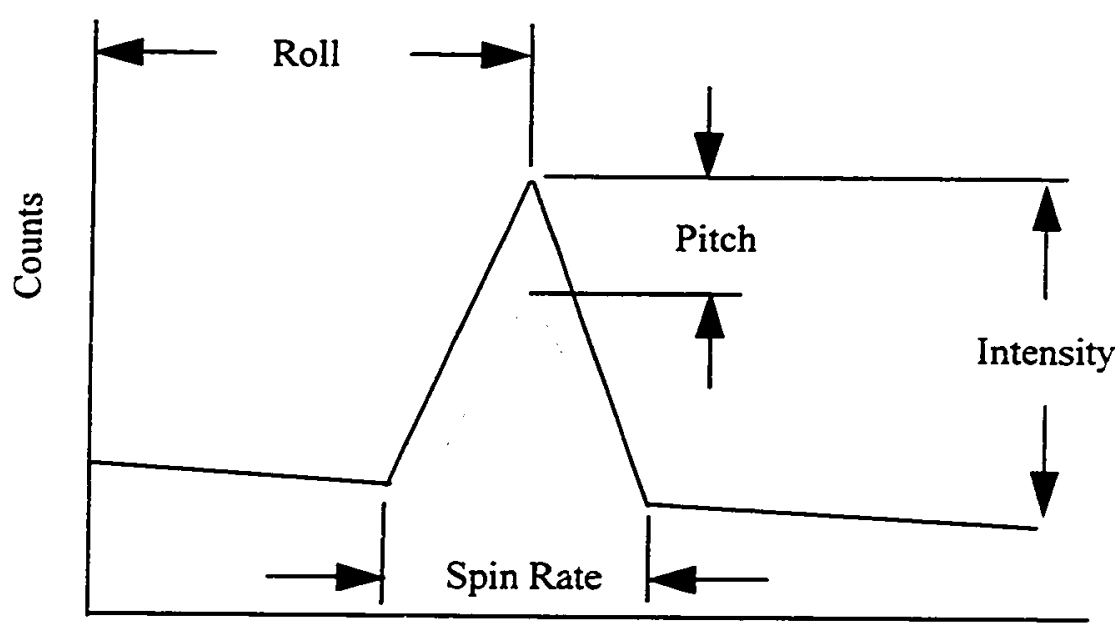

Time

Figure 4.7: Dual Detector Fit Parameters 
The two curves (one for each Module) were fitted to the data simultaneously by minimizing the Chi-Squared error. That is.

$$
\min \chi^{2}=\sum_{i=0}^{N-1}\left\{\frac{\left[Y_{3 i}(P)-C_{3 i}\right]^{2}}{\sqrt{C_{3 i}}}+\frac{\left[Y_{5 i}(P)-C_{5 i}\right]^{2}}{\sqrt{C_{5 i}}}\right\}
$$

Again, this was minimized using the IDL routine "CRVFIT" and the derivatives were computed numerically. The initial guess was generated as in Section 4.3 with three exceptions. The roll phase was initialized by using the Mod3 data alone as discussed previously. The initial intensity of the guide star was taken from the HEAO catalog. The background rates were initialized by computing the mean count rates from the two scans separately.

After solving for the set of parameters that minimize this cost function. the attitude solution was compared to the truth measurement of the spacecraft attitude. Seven scans were available where the Crab nebula passed through both the Mod3 and Mod5 collimator fields of view. The resulting improvements in the roll angle estimate. pitch angle estimate and roll rate estimate are shown in Figure 4.8.

The additional information available from Module 5 leads to a three fold improvement in the roll estimate. In particular, the standard deviation in the roll error was reduced to $0.0075 \mathrm{deg}$ while the bias dropped to $0.012 \mathrm{deg}$. This improvement is due in part to the subset of the data used for the differential x-ray star scanner studies. The small field of view on the Mod5 collimator limited the data set to scans where the guide star was at small pitch angles. This means that the linear structure that appears in the single collimator roll error data is suppressed.

The estimate of the pitch angle improved dramatically. The magnitude of the bias was reduced by a factor of four to $0.018 \mathrm{deg}$ and the standard deviation improved by an order of magnitude to $0.021 \mathrm{deg}$. This is due to the fact that the intensity of the source did not need to be known a priori. Thus. any ambiguity in the knowledge of this term did not introduce error into the pitch estimate. Note that if we examine the performance of the single collimator $\mathrm{X}$-ray star scanner in measuring the pitch angle over the small subset 

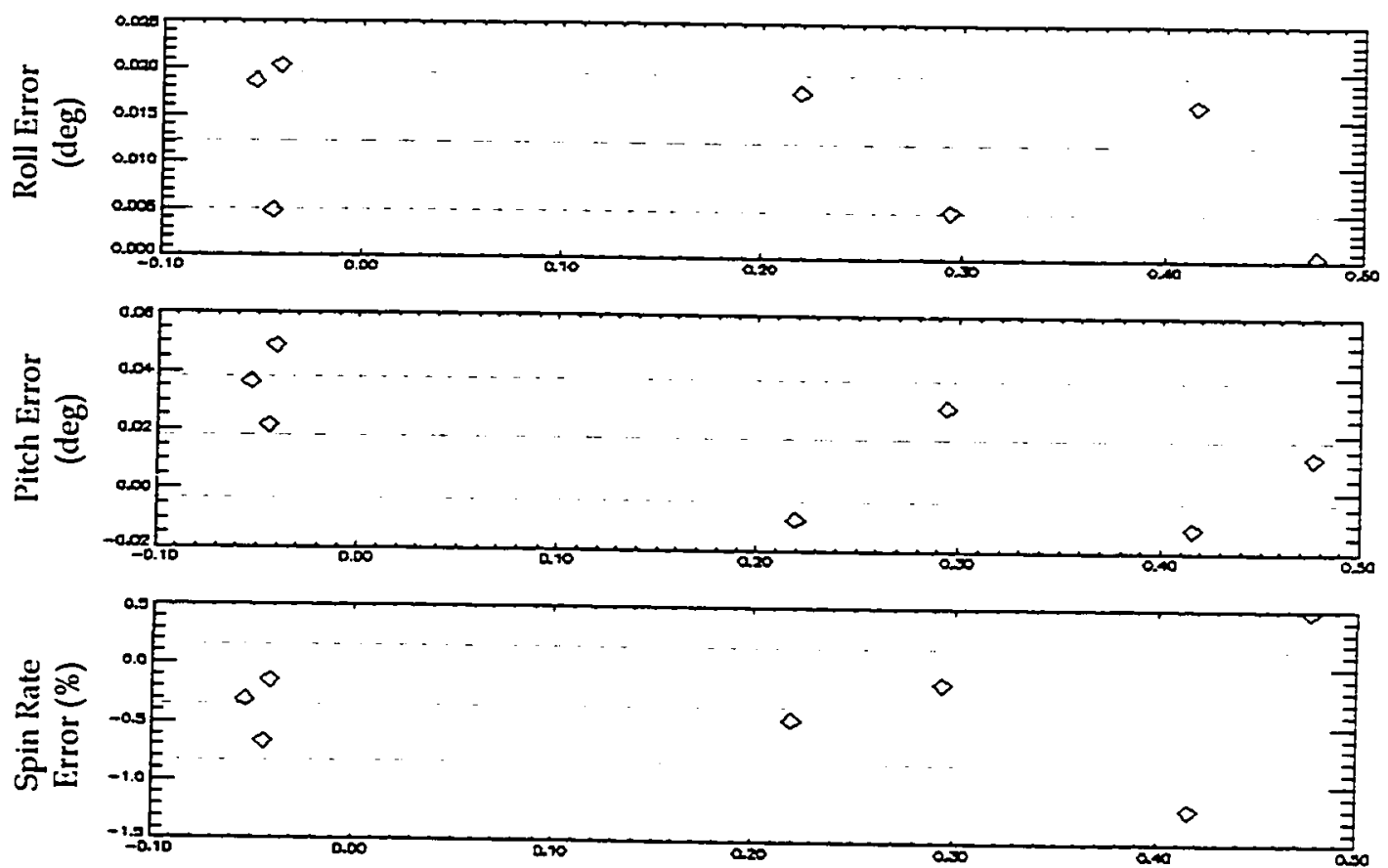

Pitch Angle (deg)

Figure 4.8: Dual Detector Performance

of scans available to the differential $x$-ray star scanner. the improvement in the differential collimator approach in seen to be even more dramatic.

The roll rate error showed a slight improvement in bias $(-0.35 \%)$ and a four-fold improvement in the standard deviation of the error $(0.48 \%)$. The addition of the guide star intensity to the model has led to an improvement in the estimate of the spin rate when the guide star is at small pitch angles. The $2 \%$ to $7 \%$ error seen in this parameter as described in Section 4.2 has been eliminated. The model can now match the large count rates consistent with small pitch angles that could not be effectively modeled before. Note also that scans with large pitch angles were not part of the subset used in this analysis.

While only seven scans were available to demonstrate the performance of differential $x$-ray star scanners, this instrument is shown to be capable of using $x$-ray 
guide stars to measure the orientation of the spacecraft with accuracies on the order of $0.01 \mathrm{deg}$.

\subsection{Dual Detector with Second Order Model}

While the performance of an x-ray star tracker using a first order model in the attitude fitting process demonstrates promising performance, there appear to be residual errors in the data that may be modelable. In particular. the roll error data from the single detector case shows a correlation between the roll error and the true pitch angle wherein the roll error appears to be linear with the pitch angle. As described in Chapter 3, the second order star scanner model predicts just such an effect. If the collimator is not properly aligned with the vehicle axes. a roll error that is proportional to the pitch angle will be introduced, viz.:

$$
\begin{aligned}
T(\rho, \alpha)=T_{\rho}(\rho) T_{\alpha}(\alpha)= & \left(1-\frac{\left|\Omega t+\delta_{z}+\left(\theta(0)+\delta_{x}\right)\left(\delta_{y}+\varepsilon_{y}\right)\right|}{\rho_{o}}\right) * \\
& \left(1-\frac{\left|\theta(0)+\delta_{x}-\theta(0) t+\left(\varepsilon_{y}+\delta_{y}\right)\left(\Omega t+\delta_{z}\right)\right|}{\alpha_{o}}\right)
\end{aligned}
$$

Note that this model predicts that the peak transmission is moved in roll linearly with the pitch angle due to the misalignment term. $\varepsilon_{y}$. The roll error shown in Figure 4.4 corresponds to a misalignment of about $1.5 \mathrm{deg}$. This happens to be approximately the size of the deadband of the attitude control system. There is also a term due to the polhode motion of the vehicle. $\theta(0)$ (described in Chapter 3 ).

The second order model was implemented in the fit process of the single detector star scanner by adding a term for the collimator misalignment and a term for the polhode motion to the parameter vector. Unfortunately, the results were not encouraging. More than half of the 99 scans available failed to converge to a solution and those that did showed a markedly worse estimate of roll and pitch than the estimates made without the 
spin axis misalignment. This is due to the following. When only one scan is available. the spin axis misalignment term, $\varepsilon_{y}$, is determined by fitting the skewness of the scan. The pitch angle is estimated from the height of the transmission peak. The roll angle estimate is then modified by the product of $\varepsilon_{y}$ and $\alpha$. Fitting the skewness of the scan is susceptible to noise and the pitch angle estimate is a function of the predicted intensity of the source. Any error in the estimate of these two parameters leads to a direct error in the estimate of the roll angle. With the pitch angle in error by as much as $0.8^{\circ}$. the roll angle estimate becomes corrupted.

By using two detectors, the estimate of the spin angle can be determined from the differential location of the peak in the scans (Figure 4.9). Since the two detectors have different pitch alignments, a misalignment of the spin axis about the nominal collimator normal will cause the peaks of the transmission curves in the two modules to shift by differing amounts. If there is no spin misalignment, the peak transmission of the two modules will occur at the same time.

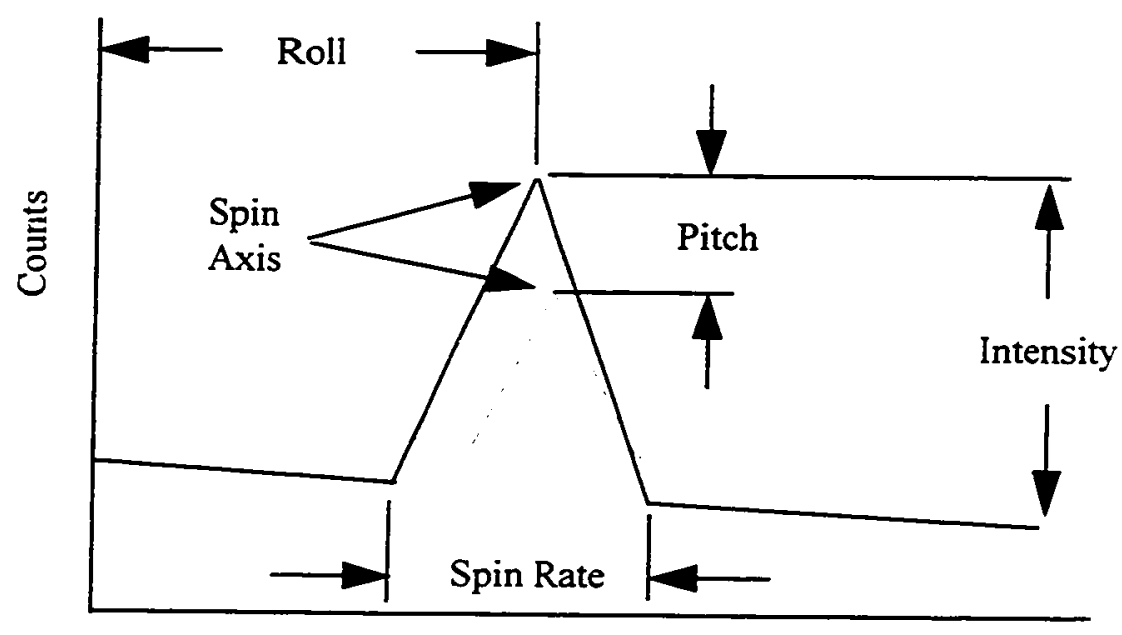

Time

Figure 4.9: Dual Detector/Second Order Model Fit Parameters 
The dual detector analysis of Section 4.4 was modified to use the second order star scanner model in the fitting process. The resulting transmission functions are.

$$
\begin{aligned}
& \mathrm{T}_{5 \alpha i}=\left(\begin{array}{r}
\tan \left|\theta(0)+\delta_{x}-\theta(0) t+\varepsilon_{y} \Omega t\right| \\
\tan \left(\alpha_{5 o}\right)
\end{array}\right) \cos \left(\theta(0)+\delta_{x}-\theta(0) t+\varepsilon_{y} \Omega t\right), \\
& \text { when }\left|\theta(0)+\delta_{x}-\dot{\theta}(0) t+\varepsilon_{y} \Omega t\right| \leq \alpha_{5 o} \\
& \mathrm{~T}_{5 \alpha i}=0, \quad \text { when }\left|\theta(0)+\delta_{x}-\dot{\theta}(0) t+\varepsilon_{y} \Omega t\right| \geq \alpha_{5 o}
\end{aligned}
$$

$$
\begin{array}{ll}
\mathrm{T}_{5 \rho i}=\left(1-\frac{\tan \left|\Omega t+\theta(0)\left(\delta_{y}+\varepsilon_{y}\right)\right|}{\tan \left(\rho_{50}\right)}\right) \cos \left(\Omega t+\left(\theta(0)+\delta_{x}\right) \varepsilon_{y}\right), \\
\mathrm{T}_{5 \rho i}=0 . & \text { when }\left|\Omega t+\theta(0)\left(\delta_{y}+\varepsilon_{y}\right)\right| \leq \rho_{5 o} \\
& \text { when }\left|\Omega t+\theta(0)\left(\delta_{y}+\varepsilon_{y}\right)\right| \geq \rho_{5 o}
\end{array}
$$

Summarizing the ten fit parameters:

1. The location of the peak transmissions is a measure of the roll phase of the vehicle $\left(i_{p}\right)$.

2. The difference in the heights of the triangles is a measure of the pitch angle of the spin axis $(\theta(0))$.

3. The absolute heights of the triangles is a measure of the intensity of the guide star (R).

4. The width of the triangles is a measure of the spin rate of the vehicle $\left(\omega_{s}\right)$. 
5. The difference in the location of the two peaks in a measure of the spin axis misalignment $\left(\varepsilon_{\mathrm{y}}\right)$.

6. The skewness of the scan is a measure of the polhode motion, $\theta(0)$

7-10. A sloped background was fit to the data from each Module $\left(B_{30}, B_{3 f}\right.$. $\mathrm{B}_{50}, \mathrm{~B}_{5 \mathrm{f}}$ ).

The parameter vector is $P=\left[B_{30}, B_{3 f}, B_{50}, B_{s f}, i_{p}, \omega_{s}, \alpha, R, \varepsilon_{y}, \dot{\theta}(0)\right]^{T}$

The two curves (one for each Module) were again fit to the data simultaneously by minimizing the Chi-Squared error and the attitude solution was compared to the true aspect solution as previously discussed. Figure 4.10 shows that the increased complexity of the second order star model does not improve the performance of the instrument. This is probably due to the reduced set of scans available to the study. The small FOV of Module 5 limited the data set to scans with a small pitch angle - the same scans that should have the smallest error in roll angle due to spin axis misalignment. The spin misalignment estimate term was generally in the one degree range while the nutation rate was a few millidegrees per second. 

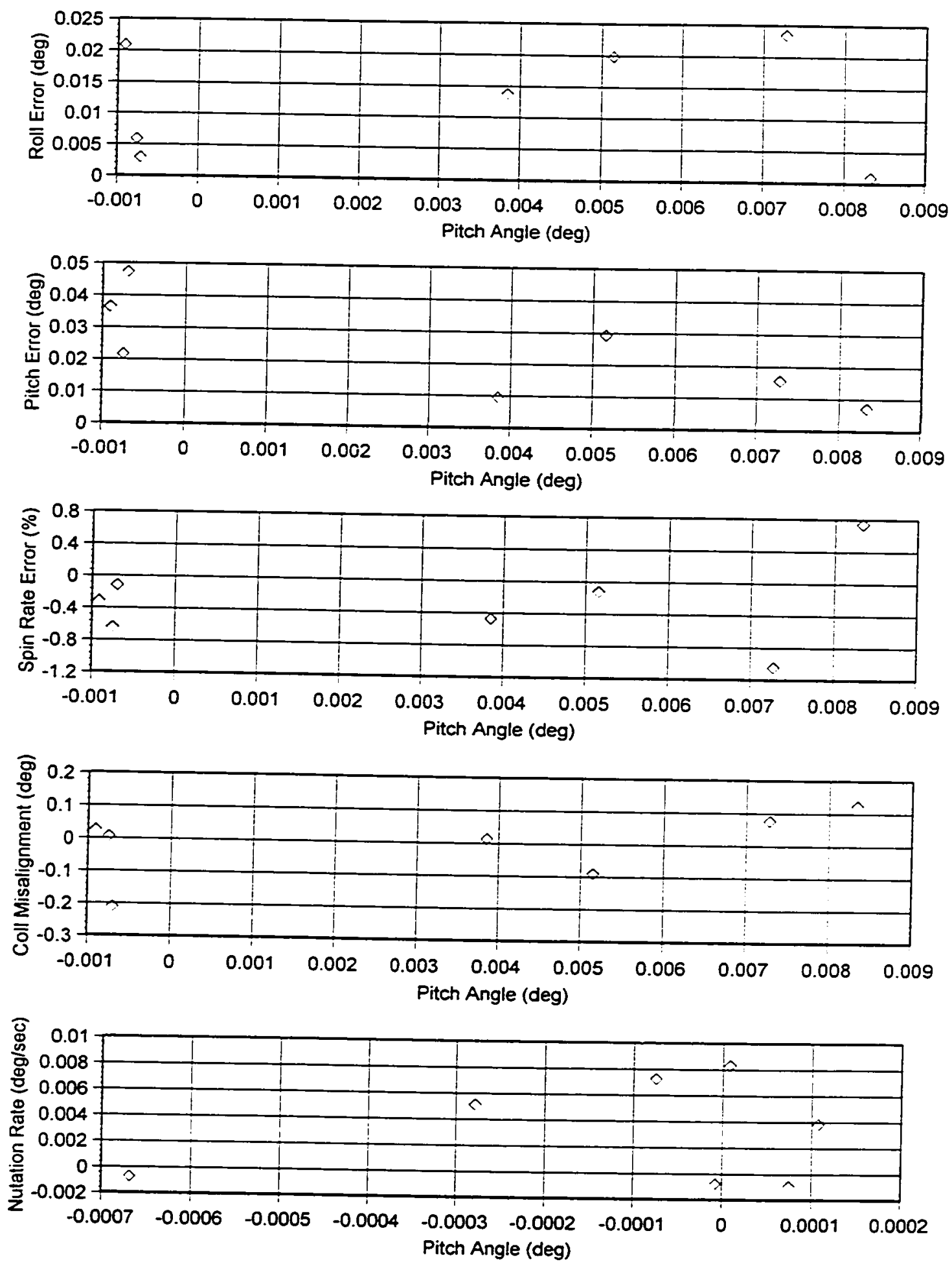

Figure 4.10: Differential Detector / Second Order Model Results 


\subsection{HEAO-A1 Attitude Determination Summary}

\begin{tabular}{|c|c|c|c|c|c|c|}
\hline \multirow{2}{*}{$\begin{array}{c}\text { Measured } \\
\text { Parameter }\end{array}$} & \multicolumn{2}{|c|}{$\begin{array}{c}\text { Single } \\
\text { Detector }\end{array}$} & \multicolumn{2}{c|}{$\begin{array}{c}c \\
\text { Detector (1st Order) }\end{array}$} & \multicolumn{2}{c|}{ Detector (2nd Order) } \\
\cline { 2 - 7 } & $\mu$ & $\sigma$ & $\mu$ & $\sigma$ & $\mu$ & $\sigma$ \\
\hline $\begin{array}{c}\text { Roll Error } \\
\text { (deg) }\end{array}$ & 0.032 & 0.030 & 0.012 & 0.0075 & 0.013 & 0.0093 \\
\hline $\begin{array}{c}\text { Pitch Error } \\
\text { (deg) }\end{array}$ & -0.084 & 0.19 & 0.018 & 0.021 & 0.024 & 0.015 \\
\hline $\begin{array}{c}\text { Roll Rate Error } \\
(\%)\end{array}$ & $-0.56 \%$ & $2.0 \%$ & $-0.35 \%$ & $0.48 \%$ & $-0.28 \%$ & $0.56 \%$ \\
\hline
\end{tabular}




\section{Chapter 5: Autonomous X-ray Time Keeping}

\subsection{Introduction}

One of the most exciting aspects of $x$-ray navigation is the prospect of using $x$-ray pulsars as frequency references. A single $\mathrm{x}$-ray pulsar can provide a stable reference for keeping local time. Since the periods of known $x$-ray pulsars vary from milliseconds to months, by observing several pulsars simultaneously an unambiguous measurement of local time can be made. That is, each pulsar will give a measure of time on a different timescale - one for seconds, one for hours, one for days and so on (Wood, 93). Unlike current methods of keeping time, this method can be used to measure local time on satellites anywhere, so long as their position is known. Furthermore, this process requires no intervention from ground support or other systems (e.g. Global Positioning System).

In this chapter, we will consider the use of a single $x$-ray pulsar (Crab Nebula pulsar) to provide a frequency reference at short timescales $\left(-10^{-2} \mathrm{sec}\right)$. 


\subsection{Phase Locked Loops}

The signal from an $\mathrm{x}$-ray pulsar cannot be used directly as a clock signal by a satellite. The pulsations have a complex shape and are imbedded in an overall signal that has a very poor signal-to-noise ratio (SNR). To be useful, the satellite clock must have a signal with a specific pulse shape (e.g. sawtooth, square, sinusoid) and phase noise low enough to meet the mission timing requirements. This local clock is typically provided by an oscillator. The frequency of the local oscillator will drift with time due to environmental effects such as changing ambient temperature, vibration and even fluctuations in the local gravitational field (Gardner, 1979). To provide accurate time information over long timescales, it is necessary to slave the oscillator to a stable reference frequency - in this case an $\mathrm{x}$-ray pulsar. In this way the long period fluctuations of the local oscillator can be removed.

While many different designs have been suggested to accomplish this, one of the most popular is to use a mechanism called the phase locked loop (PLL). A PLL is an estimator that uses a feedback loop to drive the phase difference between the reference signal (in this case the signal from the pulsar) and the signal from a voltage controlled oscillator (VCO) to zero. The VCO is a local oscillator that produces a periodic signal (square, sinusoid, sawtooth, etc.) whose frequency can be varied by an extemal signal. Furthermore, the presence of the VCO introduces a nonlinear element into the PLL that causes frequency of the VCO to be matched to the average frequency of the input signal. This allows the PLL to combine a narrow bandwidth in tracking for good noise rejection with a wide bandwidth in acquisition for robust performance.

The PLL (Figure 5.1) consists of four components:

1. Prefilter

2. Phase Detector

3. Loop Filter

4. Voltage Controlled Oscillator 


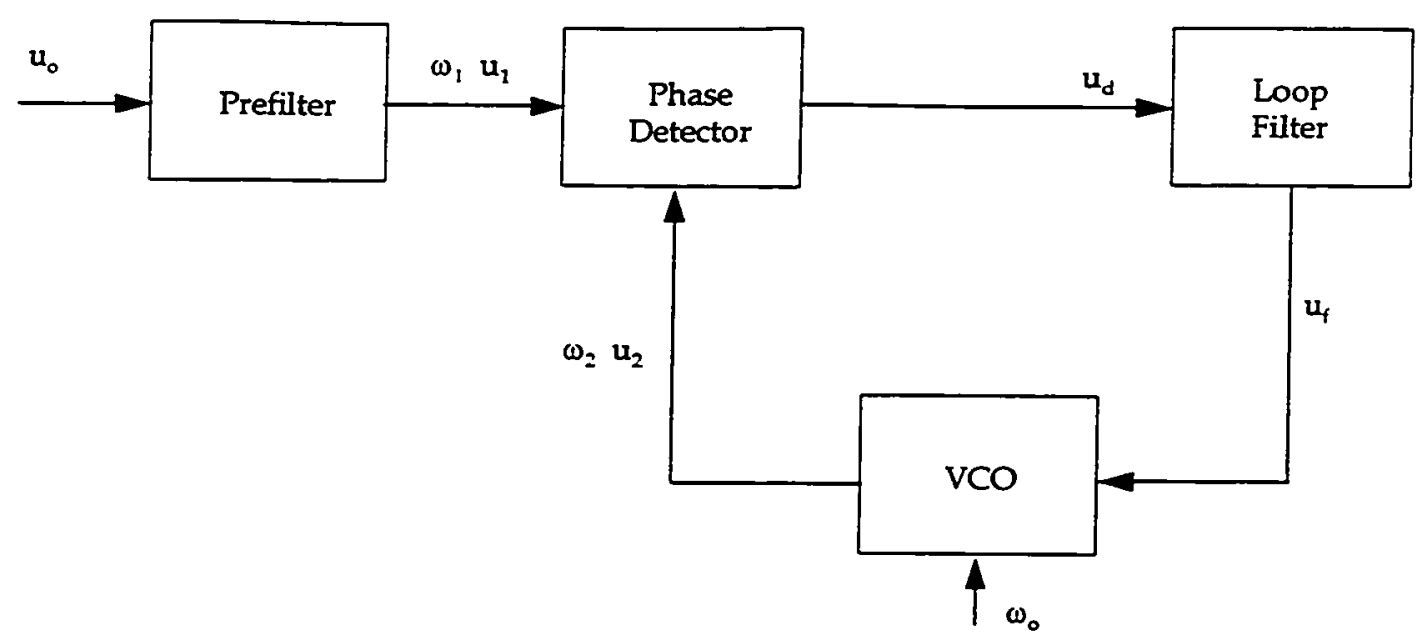

Figure 5.1: Phase Locked Loop Schematic

The reference signal $\left(u_{0}\right)$ is first passed through a prefilter. This is typically a bandpass filter with a center frequency approximately equal to the reference frequency. The passband of the prefilter must be large enough to guarantee that any drift in the reference frequency or the prefilter parameters do not cause the reference signal to become attenuated, but small enough to remove as much noise as possible. Removing the noise with a prefilter helps the performance of the PLL in both acquisition and tracking.

The prefiltered signal, $u_{1}$, is then sent to the PLL. A complete description of the modeling of the phase locked loop is contained in Appendix $\mathrm{H}$ and a host of publications (Gardner, 1979; Best, 1993). This description is summarized here.

The phase detector takes the prefiltered reference signal, $\mathrm{u}_{\mathrm{l}}$, and the VCO signal, $\mathrm{u}_{2}$, and computes the difference in phase between the two signals, viz.:

$$
\begin{aligned}
& \theta_{e}=\theta_{1}-\theta_{2} \\
& u_{d}=K_{p d} \theta_{e}
\end{aligned}
$$

This phase error, $u_{d}$, is sent to its output. Ideally, the phase error would be computed directly. Unfortunately, $u_{1}$ and $u_{2}$ are typically sine waves - not direct phase measurements, so the phase error cannot be computed by simple subtraction. One way 
to compute the phase difference between two signals is to multiply them together. Consider the simplest case where $u_{1}$ and $u_{2}$ are sine waves, but $90^{\circ}$ out of phase:

$$
\begin{aligned}
& u_{1}=\sin \left(\theta_{1}\right) \\
& u_{2}=\cos \left(\theta_{2}\right) \\
& u_{d}=K_{p d} u_{1} u_{2}
\end{aligned}
$$

It can be shown (Appendix $\mathrm{H}$ ) that for small phase errors the detector signal is,

$$
u_{d} \cong K_{p d} \theta_{e}+\text { terms at twice the reference frequency }
$$

If this signal is passed through a loop filter that suppresses signals at twice the input frequency, the phase detector can be approximated as ideal. This implies that the loop is operating in a locked state. That is, the VCO frequency, $\omega_{2}$, is the same as the reference frequency, $\omega_{1}$.

One of the roles of the loop filter is to suppress the errors in the performance of the phase detector. Additionally, the loop filter is the primary factor in determining the performance of the PLL, in both tracking and acquisition modes. The literature is extensive on this point (Gardner, 1979). Many methods exist to design filters of varying complexity. Unfortunately, analytical description of the nonlinear acquisition of PLL operations exist only for PLL's with a few specific loop filters. In fact, it is difficult to guarantee the dynamic stability of loops with filters of higher than second order. Two loop filters whose performance have been described analytically are:

$$
\begin{array}{ll}
\text { Active Lag-Lead: } & F_{l f}(s)=K_{f} \frac{1+\tau_{2} s}{1+\tau_{1} s} \\
\text { Proportional + Integral Control: } & F_{l f}(s)=\frac{1+\tau_{2} s}{\tau_{1} s}
\end{array}
$$

While the types of loop filters available are as varied as the applications of PLL's, these two filters provide an excellent set of design options while being simple enough to allow accurate prediction of their performance in acquiring and tracking a complex signal in the presence of noise (Gardner, 1979). 
The voltage controlled oscillator is the local clock that will be used as the frequency reference by the satellite. The signal from the loop filter $\left(u_{f}\right)$ is used to control the frequency of the $\mathrm{VCO}$,

$$
\omega_{v c o}(t)=\omega_{0}+K_{v c o} u_{f}(t)
$$

The phase of the $\operatorname{VCO}\left(\theta_{2}\right)$, is just the integral of $\omega_{v c o}$,

$$
\begin{aligned}
& \theta_{2}(t)=\int_{0}^{t} \omega_{v c o}(t) d t+\theta_{2}(0) \\
& \Theta_{2}(s)=\frac{K_{v c o}}{s} U_{f}(s) \\
& u_{2}(t)=\cos \left(\theta_{2}(t)\right)
\end{aligned}
$$

Note that when the VCO is tracking the reference signal $\left(\theta_{e}\right.$ is small), the output of the $\mathrm{VCO}, \mathrm{u}_{2}$, is actually $90^{\circ}$ out of phase with the reference.

The general nonlinear equations for a phase locked loop are.

$$
\begin{aligned}
& U_{\mathrm{l}}(s)=F_{p f}(s) U_{0}(s) \\
& u_{d}(t)=K_{p d} u_{1}(t) u_{2}(t) \quad F_{p f}(s)=\text { Prefilter Transfer Function } \\
& U_{f}(s)=F_{l f}(s) U_{d}(s) \quad K_{p d}=\text { Phase detector Gain } \\
& \omega_{v c o}(t)=\omega_{0}+K_{v c o} u_{f}(t) \quad \text { where, } F_{l f}(s)=\text { Loop Filter Transfer Function } \\
& \theta_{2}(t)=\int_{0}^{t} \omega_{v c o}(t) d t+\theta_{2}(0) \quad \omega_{0}=\text { VCO Center Frequency } \\
& K_{v c o}=\text { VCO Gain } \\
& u_{2}(t)=\cos \left(\theta_{2}(t)\right)
\end{aligned}
$$

If the loop is in the locked state, the equations for the phase-locked loop can be linearized in terms of the phase of reference signal and the VCO as (Best. 1993),

$$
\begin{aligned}
& H(s)=\frac{\Theta_{2}(s)}{\Theta_{1}(s)}=\frac{K_{v c o} K_{p d} F(s)}{s+K_{v c o} K_{p d} F(s)} \\
& H_{e}(s)=\frac{\Theta_{e}(s)}{\Theta_{1}(s)}=\frac{s}{s+K_{v c o} K_{p d} F(s)}
\end{aligned}
$$


Note that the performance of the loop, being generally defined by its closed loop poles, is a function of the detector gain $\mathrm{K}_{\mathrm{pd}}$. Since $\mathrm{K}_{\mathrm{pd}}$ is a function of the amplitude of the input signal, variations in this amplitude or errors in predicting its size will affect the dynamics of the loop. This is demonstrated further in the PLL simulation discussed in Section 5.5.

Before the PLL can get into its locked state, it must undergo acquisition. In this process the nonlinear characteristics of the loop cause it to search for the frequency of the input signal. For example, if the VCO frequency is slower than the input signal frequency, over a cycle the input signal will lead the VCO signal causing a positive phase error. This is multiplied by the filter gain and ends up as a positive signal to the input of the VCO increasing the frequency of the VCO until it catches the input signal and locks.

There are several regimes of operation in which the nonlinear characteristics of the loop are important (Best, 1993). These ranges are generally described by a frequency offset about the nominal VCO frequency (Figure 5.2). The "Hold Range" is that frequency range where once the loop becomes locked, it will remain locked if no disturbances are present. This is the static stability limit of the loop. The "Pull-in Range" defines the dynamic stability limit of the loop. If the loop is unlocked, but the frequency offset is within the pull-in range, the loop will eventually lock-in. A subset of the pull-in range is the "Lock Range". The lock range defines a range of frequency offset inside which the loop will lock onto the reference signal within one beat note of the reference and VCO signals. That is, there will be no cycle slips before the PLL acquires the reference signal. Most loops are designed such that any conceivable initial frequency offset will be within the lock range. The acquisition performance of phase-locked loops with simple loop filters has been analyzed (Best, 1993). The performance of three simple loops are summarized in Table 5.1. 


\begin{tabular}{|l|c|c|}
\hline \multicolumn{1}{|c|}{ Loop Type } & Active Lag-Lead & $\begin{array}{c}\text { Proporional - Integral } \\
\text { Control }\end{array}$ \\
\hline Transfer Function & $F_{l y}(s)=K, \frac{1+\tau_{2} s}{1+\tau_{1} s}$ & $F_{v}(s)=\frac{1+\tau_{2} s}{\tau_{i} s}$ \\
\hline Loop Natural Frequency & $\omega_{n}=\frac{\overline{K_{v c o} K_{p d} K_{f}}}{\tau_{1}}$ & $\omega_{n}=\frac{K_{v c o} K_{p i}}{\tau_{1}}$ \\
\hline Loop Damping & $\zeta=\frac{\omega_{n}}{2}\left(\tau_{2}+\frac{1}{K_{v c o} K_{p d} K_{f}}\right)$ & $\zeta=\frac{\omega_{n} \tau_{2}}{2}$ \\
\hline Hold Range & $\Delta \omega_{H}=K_{v c o} K_{p d} K_{f}$ & $\Delta \omega_{H}=\infty$ \\
\hline Pull-In Range & Complex & $\Delta \omega_{P}=\infty$ \\
\hline Lock Range & $\Delta \omega_{L}=K_{f} \frac{\tau_{2}}{\tau_{1}}$ & $\Delta \omega_{L}=\frac{\tau_{2}}{\tau_{1}}$ \\
\hline Lock Time & $T_{L}=\frac{2 \pi}{\omega_{n}}$ & $T_{L}=\frac{2 \pi}{\omega_{n}}$ \\
\hline
\end{tabular}

Table 5.1: PLL Characteristics

\subsection{Error Sources}

Several factors can introduce errors into timing measurements that are made from $\mathrm{X}$-ray pulsars.

\section{Poisson Fluctuations}

The dominant source of noise in an x-ray pulsar is the photon noise associated with the low count rates of these sources and appears to a PLL as a phase error. The purpose of the PLL is to reduce this error to an acceptable level. 


\section{Phase Detector Offset}

The same non-linearities that give the phase-locked loop its adaptive characteristics also cause it to have a steady-state error in phase, even when the loop filter has an integral term that should drive such errors to zero. This is due to the multiplying effect of the phase detector and is a function of such measurable parameters as signal strength, detector and VCO gain and loop filter frequency response. It should be possible to describe this error analytically and remove it.

The complexity of the pulse shape will also introduce an offset in the time measurement as the loop will not lock onto the front of the pulse, but will lock in phase with the fundamental of the pulsation. This offset can be predicted analytically and removed as well.

\section{Position-Timing Error}

An error in the measurement of the position of a satellite will cause an error in the measurement of absolute time and, if fluctuations in position over short time scales are large enough, will cause errors in local time measurements by way of an apparent modulation of the reference frequency. Consider two detectors in two different positions measuring time from the same pulsar. If the distance traveled by the pulse wavefront from the star to each detector is different, the trailing detector will be measuring a time behind the leading detector, viz.:

$$
\Delta t=\frac{\Delta x}{c}
$$

Thus, an error in the measured position of the satellite relative to some "inertial" point (i.e. the barycenter of the earth-sun system) of $300 \mathrm{~m}$ will cause an error in the inertial time measurement of $1 \mathrm{~ms}$. Known satellite displacements (e.g. as the satellite orbits the earth and the earth orbits the sun) can be taken out of the measurements predictively to attain inertial measurements of time. 


\section{"Free-riding Zones"}

At times a pulsar may not be available to a satellite (e.g. when the earth, sun or moon occludes the reference pulsar from the satellite). At these times the clock will be running at the last frequency update from a locked condition. This error cannot be removed from the measurements, but its magnitude can be bounded before the mission. Alternately, the clock can shift from one pulsar to the next as it orbits the earth.

\section{Pulse Frequency Knowledge}

The measurement of time is only as good as the reference. If there is error in the knowledge of the pulsar pulse period, this will be propagated into the absolute time measurement. Consider a detector that is locked onto a pulsar that has a pulse frequency of $10 \mathrm{~Hz}$, but the knowledge of that pulse period is only good to a part in $10^{9}$. If the actual pulse period of the frequency reference is $10+10^{-8}$, the local clock will run fast and the local time will diverge from absolute time. These errors cannot be removed, but they can be bounded.

\section{Spin Down of X-ray Pulsars}

The spin rates of pulsars are known to be increasing. These accelerations are very small and are systematic. They have been measured or bounded so that they can be removed from the measurements.

\section{Local Oscillator Fluctuations}

The local oscillator is not a perfect instrument, otherwise it would not need to be slaved to an $x$-ray source. Small variations in its frequency will increase the error in the local time. Additionally, if the local oscillator is used to time-tag the detector photons, this may cause an error in the measured signal. 


\section{Random Fluctuations of X-ray Pulsars}

Some X-ray pulsars are known to have occasional random jumps in phase. If multiple sources are used for timing information, a fluctuation in one pulsar should be observable.

\section{Doppler Shift of Pulsar Frequency}

The moition of the detector along the line of sight to the reference object will cause the frequency of the pulsation to be Doppler shifted. This will cause a frequency error in the detector that is periodic with the orbit:

$$
\Delta f=\frac{v_{\text {los }}}{c} f_{\text {puilsar }}
$$

where $v_{\text {los }}$ is the velocity of the satellite along the line of sight to the pulsar.

This error will not grow with time and can be bounded and even removed to the level that the velocity of the satellite is known. Additionally, by observing multiple pulsars simultaneously, it should be possible to remove any long-term effects of the Doppler shift.

\subsection{Simulation Description}

To demonstrate the feasibility of lising $x$-ray pulsars as inertial time references, the performance of a phase-locked loop was simulated. This simulation included the acquisition phase. Anticipating the USA mission, it was assumed that the HEAO catalog described the overall intensity of $\mathrm{x}$-ray sources and that the response of the $\mathrm{x}$-ray detector could be modeled as a HEAO detector (including the geometric area). This assumption also anticipates the availability of data from the pointed portions of the HEAO data. The results of this simulation should predict to some degree the performance of a PLL on HEAO pointed data as well as on a real-time USA mission. 


\begin{tabular}{|c|c|c|c|c|}
\hline Source & $\begin{array}{c}\text { Alternate } \\
\text { Name } \\
\end{array}$ & $\begin{array}{c}\text { Pulse Period } \\
\text { (sec) }\end{array}$ & Type & $\begin{array}{c}\text { Year of } \\
\text { Discovery }\end{array}$ \\
\hline & Crab Nebula & 0.033 & & \\
\hline $\mathrm{X} 0535-668$ & A0538-66 & 0.069 & Transient & 1982 \\
\hline $\mathrm{X} 0115-737$ & SMC X-1 & 0.71 & Transient & 1976 \\
\hline $\mathrm{X} 1656+354$ & Her X-1 & 1.24 & Variable & 1972 \\
\hline $\mathrm{X} 0115+634$ & V625 Cas & 3.6 & Transient & 1978 \\
\hline $\mathrm{X} 0332+530$ & BQ Cam & 4.4 & Transient & 1985 \\
\hline X1119-603 & Cen X-3 & 4.8 & & 1971 \\
\hline $\mathrm{X} 1048-594$ & & 6.4 & & 1990 \\
\hline $\mathrm{X} 2259+587$ & & 7.0 & & 1980 \\
\hline $\mathrm{X} 1627-673$ & & 7.7 & & 1977 \\
\hline $\mathrm{X} 1553-542$ & & 9.3 & & 1983 \\
\hline $\mathrm{X} 0834-430$ & GR0834-430 & 12.2 & Transient & 1991 \\
\hline $\mathrm{X0532-664}$ & LMC X-4 & 13.5 & Variable & 1983 \\
\hline $\mathrm{X} 1417-624$ & & 17.6 & Transient & 1981 \\
\hline $\mathrm{X} 1843+009$ & & 29.5 & & 1990 \\
\hline $\mathrm{X} 1657-415$ & & 38 & & 1979 \\
\hline $\mathrm{X} 2030+375$ & & 42 & & 1989 \\
\hline $\mathrm{X} 2138+568$ & Cep X-4 & 66 & & 1991 \\
\hline $\mathrm{X} 1836-045$ & & 81 & & 1990 \\
\hline $\mathrm{X} 1843-024$ & & 95 & & 1990 \\
\hline $\mathrm{X} 0535+262$ & & 104 & Transient & 1975 \\
\hline $\mathrm{X} 1833-076$ & Sct X-1 & 111 & & 1991 \\
\hline $\mathrm{X} 1728-247$ & GX $1+4$ & 114 & & 1971 \\
\hline $\mathrm{X} 0900-403$ & Vela X-1 & 283 & & 1976 \\
\hline $\mathrm{X} 1258-613$ & GX 304-1 & 272 & & 1977 \\
\hline $\mathrm{X} 1145-614$ & & 298 & & 1978 \\
\hline $\mathrm{X} 1145-619$ & & 292 & & 1978 \\
\hline $\mathrm{X} 1118-615$ & Al118-61 & 405 & Transient & 1975 \\
\hline $\mathrm{X} 1722-363$ & & 413 & & 1989 \\
\hline $\mathrm{X} 1907+097$ & & 438 & & 1984 \\
\hline X1538-522 & QV Nor & 529 & & 1977 \\
\hline $\mathrm{X} 1223-624$ & GX 301-2 & 696 & Transient & 1976 \\
\hline $\mathrm{X0352-309}$ & X Per & 835 & & 1990 \\
\hline
\end{tabular}

Table 5.2: Pulsar Characteristics (White, 1995) 
There are a number of pulsars that could be used as time references. Some of these are summarized in Table 5.2. The pulsar at the center of the Crab Nebula has one of the shortest periods yet discovered in an x-ray pulsar. It is one of the brightest sources in the $\mathrm{x}$-ray sky in terms of total flux and has been studied extensively for a number of years. This source was also used as a guide star in the attitude determination portion of this thesis. For these reasons, the Crab Nebula was chosen as the pulsar reference for the inertial time reference simulation.

The Einstein mission was able to characterize the pulsar in the Crab Nebula quite extensively (Harnden, 1984). The pulse shape observed by Einstein in shown in Figure 5.2. Over a single period these pulsations account for about $4 \%$ of the total flux from the Crab. When pointed directly at the Crab, the rest of the signal comes from the steady flux from the nebula and from background (diffuse x-ray and trapped particles). The steady flux from the nebula is the primary contributor to the noise on the received signal. The signal from a pulsar at time step i can be written as,

$$
\begin{array}{cl}
C_{i}=B_{i}+S_{i}+P_{i}+N_{i} & \\
& B_{i}=\text { Counts from Background } \\
& S_{i}=\text { Counts from Steady Part of Source } \\
& P_{i}=\text { Counts from Pulsar } \\
N_{i} & =\text { Counts from Noise }
\end{array}
$$

The pulsar was modeled by fitting a composite curve of four exponentials to the Einstein data. Each sub-pulse was modeled by a rising and a falling exponential and the resulting curves were added together. The location of the two peaks were fixed at bins 94 and 222. The two curves use to fit the two peaks are,

$$
\begin{array}{ll}
P_{1 i}=a_{1}\left(e^{b_{1} i}-1\right) & i<94 \\
P_{2 i}=a_{3}\left(e^{b_{3} i}-1\right) & i<222 \\
P_{1 i}=a_{2}\left(e^{b_{2}(188-i)}-1\right) & i \geq 94 \\
P_{1 i}=a_{4}\left(e^{b_{4}(444-i)}-1\right) & i \geq 222
\end{array}
$$




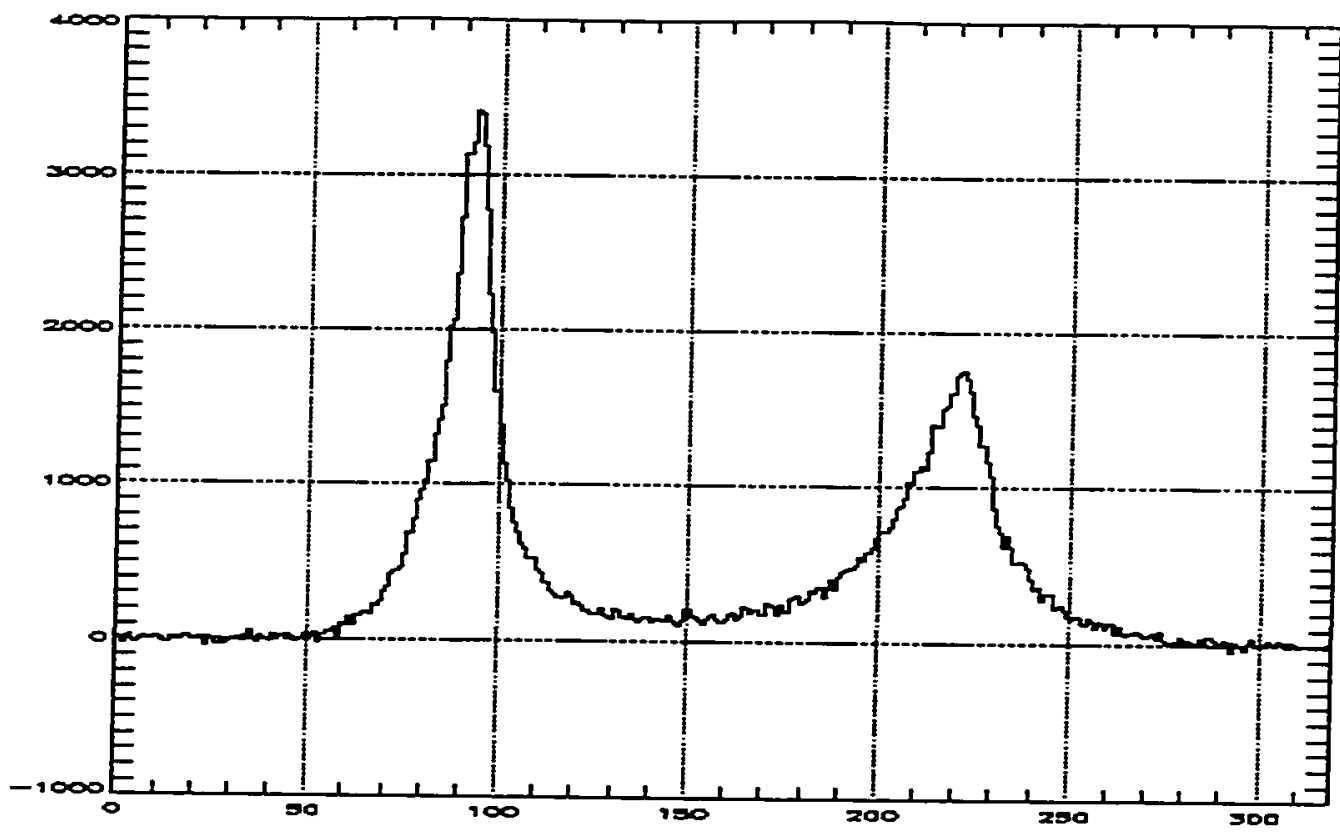

Figure 5.2: Observed Crab Pulse Shape

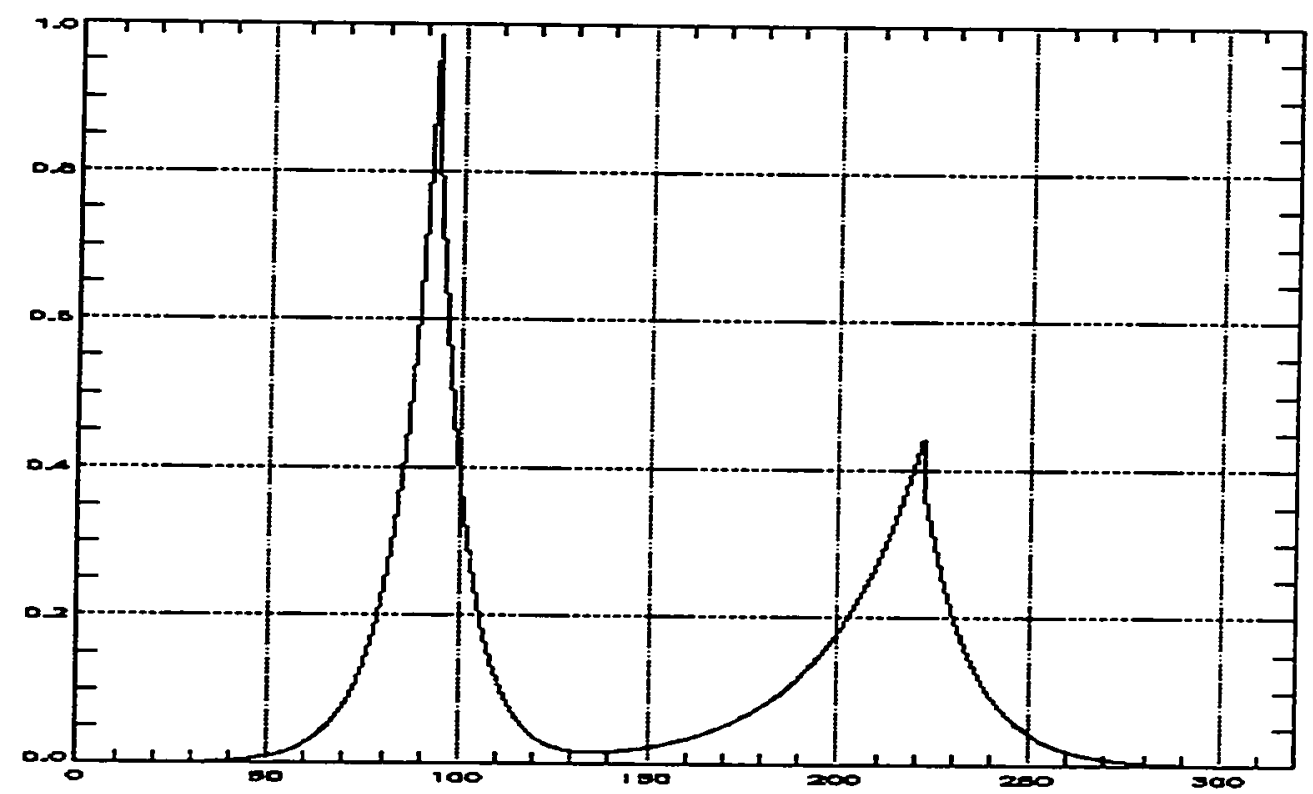

Figure 5.3: Crab Pulsar Fitted Shape 
The parameters $\left(a_{1}, b_{1}, a_{2}, b_{2}, a_{3}, b_{3}, a_{4}, b_{4}\right)$ were fit to the data by minimizing the Chisquared error (Figure 5.3). The total number of pulsar counts at a particular bin is then ( $\eta_{c}$ is a normalizing parameter to get the proper ratio of pulsar counts to total counts),

$$
P_{i}=\left(P_{1 i}+P_{2 i}\right) \eta_{c}
$$

When the size of the time bin is large compared to the features in the pulse shape, it is necessary to integrate the instantaneous count rate from the model over the total bin time. This has the effect of lowpass filtering the pulsar signal and was necessary in this simulation.

The noise present in the received signal is Poisson and can be added by using the POIDEV procedure in IDL. The mean of the signal is just the calculated signal level. For analysis purposes this noise can be approximated as Gaussian with zero mean and standard deviation,

$$
\sigma_{i}=\sqrt{B_{i}+S_{i}+P_{i}}
$$

The signal to noise ratio can then be approximated as,

$$
S N R=\frac{P_{i}}{\sqrt{B_{i}+S_{i}+P_{i}}} \cong \frac{P_{i}}{\sqrt{B_{i}+S_{i}}}
$$

\subsection{Simulated PLL Design}

While it is not the purpose of this simulation to optimize the performance of the phase locked loop in tracking the Crab pulsar, it is necessary to synthesize a design that will yield reasonable results. The first step in the design of the PLL is to determine the rate at which the reference pulsar will be sampled. This sets the bin size for the input signal, $\mathrm{u}_{0}$. There are tradeoffs to be made in selecting a bin size. The smaller the bin size, the more points sampled per pulse and the better the representation of the pulse. But the noise in a given bin is inversely proportional to the square-root of the bin size. Thus, the smaller the bin size, the greater the noise on the signal. A large bin size was used to 
reduce the level of noise on the signal, but at $5\left(10^{-3}\right) \sec (5 \mathrm{msec})$ sampling, several samples were taken per pulsation.

A series of simulated Crab data binned at $5 \mathrm{msec}$ was generated based on the fitted pulsar curve. The mean rate from the steady portion of the Crab nebula was subtracted. A residual of the steady portion of the signal was left in the reference signal to simulate the uncertainties in the knowledge of this parameter. This was passed through a prefilter to eliminate any remaining bias in the signal and to reduced the level of noise in the signal to help the PLL gain and maintain lock. A second order Butterworth bandpass filter was designed in MATLAB using the "BUTTER" command (MATLAB, 1995). A Butterworth filter is an optimal filter that has the property that its passband is flat with zero gain. The size of the passband can be specified when constructing the filter. This must be large enough that any error in the expected input frequency will not place the fundamental of the pulsar signal outside the passband, resulting in a reduction of signal strength. The passband must be small enough. though. to reduce the noise on the signal as much as possible. It also suppresses the harmonics inherent in a pulse shape as complex as the Crab. The passband of the prefilter used here is $+1-1 \%$ of the center frequency. The state space equations are contained in Appendix $\mathrm{H}$.

The primary driver in determining the performance of the PLL is the design of the loop filter. An active lag loop filter was chosen since it has the most free parameters (3). There are two opposing goals in the performance of the PLL - good steady state noise rejection and fast acquisition time. It turns out that to get good noise rejection, a loop should have a low natural frequency, but to get fast acquisition it should have a high natural frequency. The maximum acquisition time is limited by the length of time that a pulsar can be tracked without interruption. For example. the USA Experiment can track a pulsar for no more than $1200 \mathrm{sec}(20 \mathrm{~min})$. On the other hand, the minimum acquisition time is limited by the desired accuracy of the local clock. These competing requirements were resolved by running the loop as slowly as possible while still acquiring the signal in a single USA pass - less than $1000 \mathrm{sec}$. The natural frequency $\left(\omega_{n}\right)$ of the loop was set at $0.01 \mathrm{~Hz}$. The noise rejection is relatively insensitive to the closed loop damping $(\xi)$ 
in the neighborhood of its optimum - the critical damping ratio. Thus, the loop damping was set to 1.0 .

Given the desired overall loop parameters $\left(\omega_{n}, \xi, K_{\text {loop }}\right)$, the loop filter can be designed in terms of the two crossover frequencies, $\tau_{1}$ and $\tau_{2}$ as follows,

$$
\begin{gathered}
\tau_{1}=\frac{K_{\text {loop }}}{\omega_{n}^{2}} \\
\tau_{2}=\frac{2 \xi}{\omega_{n}}
\end{gathered}
$$

The loop gain $\left(\mathrm{K}_{\text {loop }}\right)$ determines the VCO gain $\left(\mathrm{K}_{\mathrm{veo}}\right)$ as follows,

$$
K_{v c o}=\frac{K_{l o o p}}{K_{p d} h_{i n p}}
$$

The phase detector gain $\left(\mathrm{K}_{\mathrm{pd}}\right)$ is chosen such that the total gain through the phase detector $\left(K_{\mathrm{pd}} \mathrm{h}_{\mathrm{inp}}\right)$ is unity.

The loop performance parameters can then be calculated as indicated in Table 5.1.

\subsection{Simulation Results}

The performance of the phase locked loop was simulated with the following inputs:

$$
\begin{array}{ll}
\text { VCO Frequency Offset: } & 0.01 \% \\
\text { Mean Background Error: } & 1 \% \\
\text { Initial Phase Error: } & 0 \mathrm{deg}
\end{array}
$$

The clock error is shown as a function of time in Figure 5.4 for a $2000 \mathrm{sec}$ run. Note that the PLL initially runs ahead of the reference due to the initial frequency error. The loop begins to slow the VCO down at about $50 \mathrm{sec}$ and is tracking the reference clock by $500 \mathrm{sec}$. The first $500 \mathrm{sec}$ or so is the acquisition phase. For the remaining $1500 \mathrm{sec}$, the loop is tracking its reference. On average the VCO is $0.069 \mathrm{sec}$ behind the reference and is generally within $0.0015 \mathrm{sec}$ of this mean. This offset is not entirely a 
error since the location of the zero phase point for the Crab pulse shape is arbitrary. The fundamental is not necessarily in phase with this selection. Since the PLL locks to the fundamental of the pulse shape, it will not necessarily lock to a zero phase offset.

Figure 5.5 shows a comparison of three signals within the loop: the raw input, the prefiltered signal and the VCO signal. Note that the raw signal cannot be used in any sense as a clock. The prefiltered signal at least appears sinusoidal, although there is a lot of amplitude and certainly some phase modulation of the signal. The tight bandwidth of the PLL yields the sinusoidal signal from the VCO shown in the bottom plot. The zero crossings can be used from this signal to determine time directly. Alternately, the phase measure from the simulation can be used as a time reference for higher update rates.

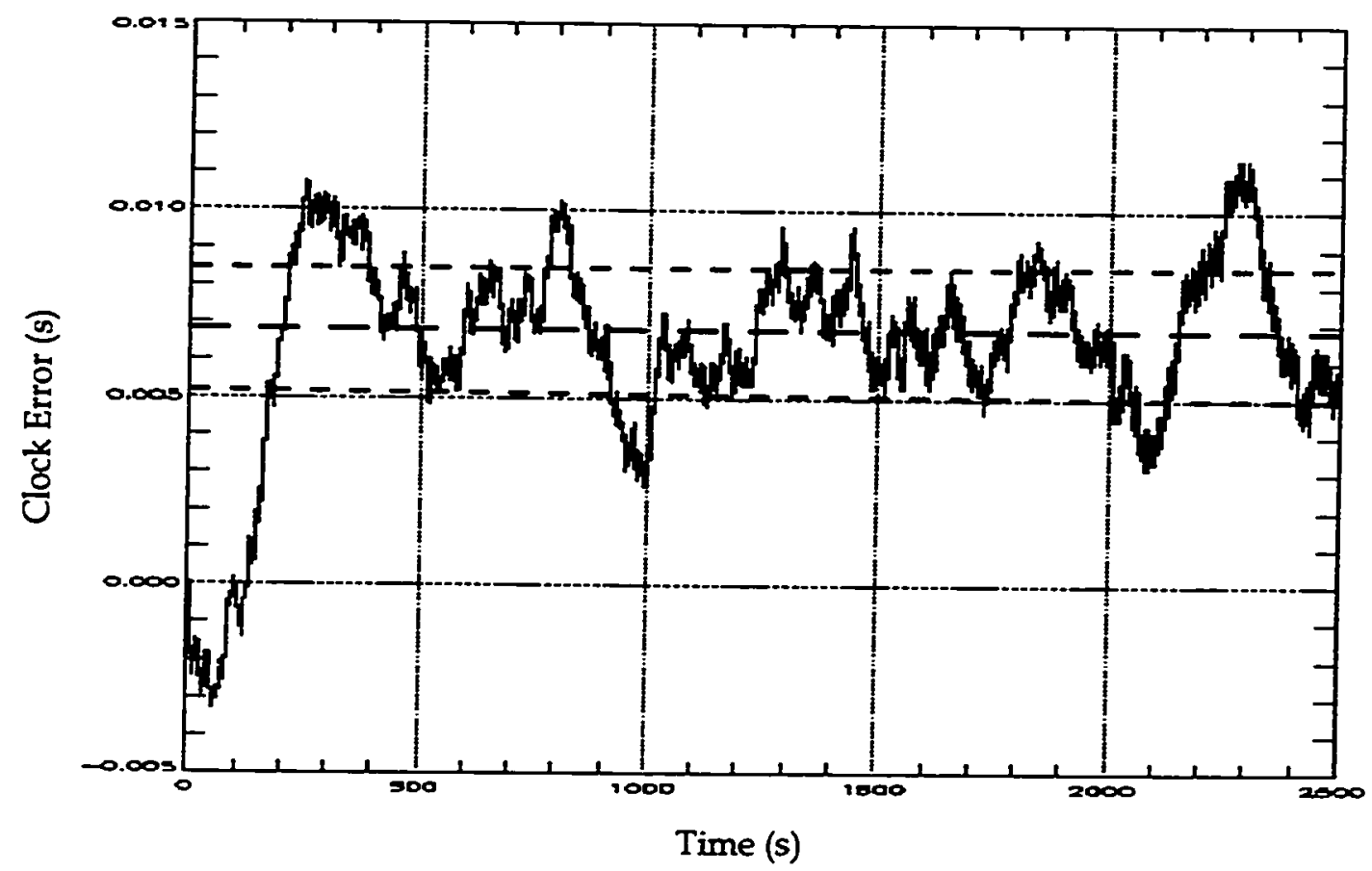

Figure 5.4: PLL Simulated Performance 


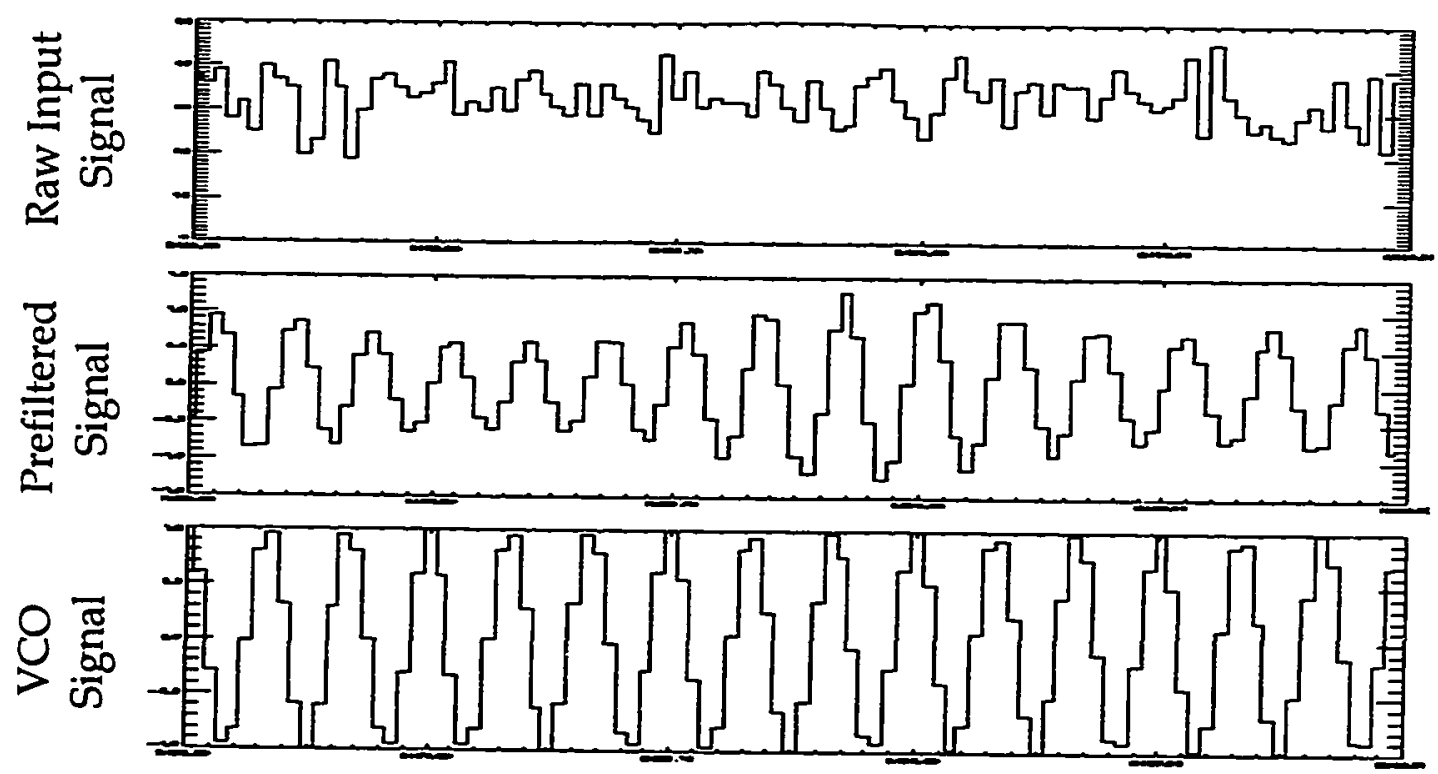

Time (s)

Figure 5.5: PLL Signal Comparison

It is possible that the simulated PLL did not lock onto any signal at all. It may have been able to lock onto noise that, after prefiltering, just appeared to be an input signal at the Crab frequency. In order to guarantee that the PLL simulation actually locked onto a real signal, the simulation was rerun with one exception - the pulse signal amplitude was set to zero. Figure 5.6 shows that the result is a loop that never achieves lock and simply drifts off in time. 


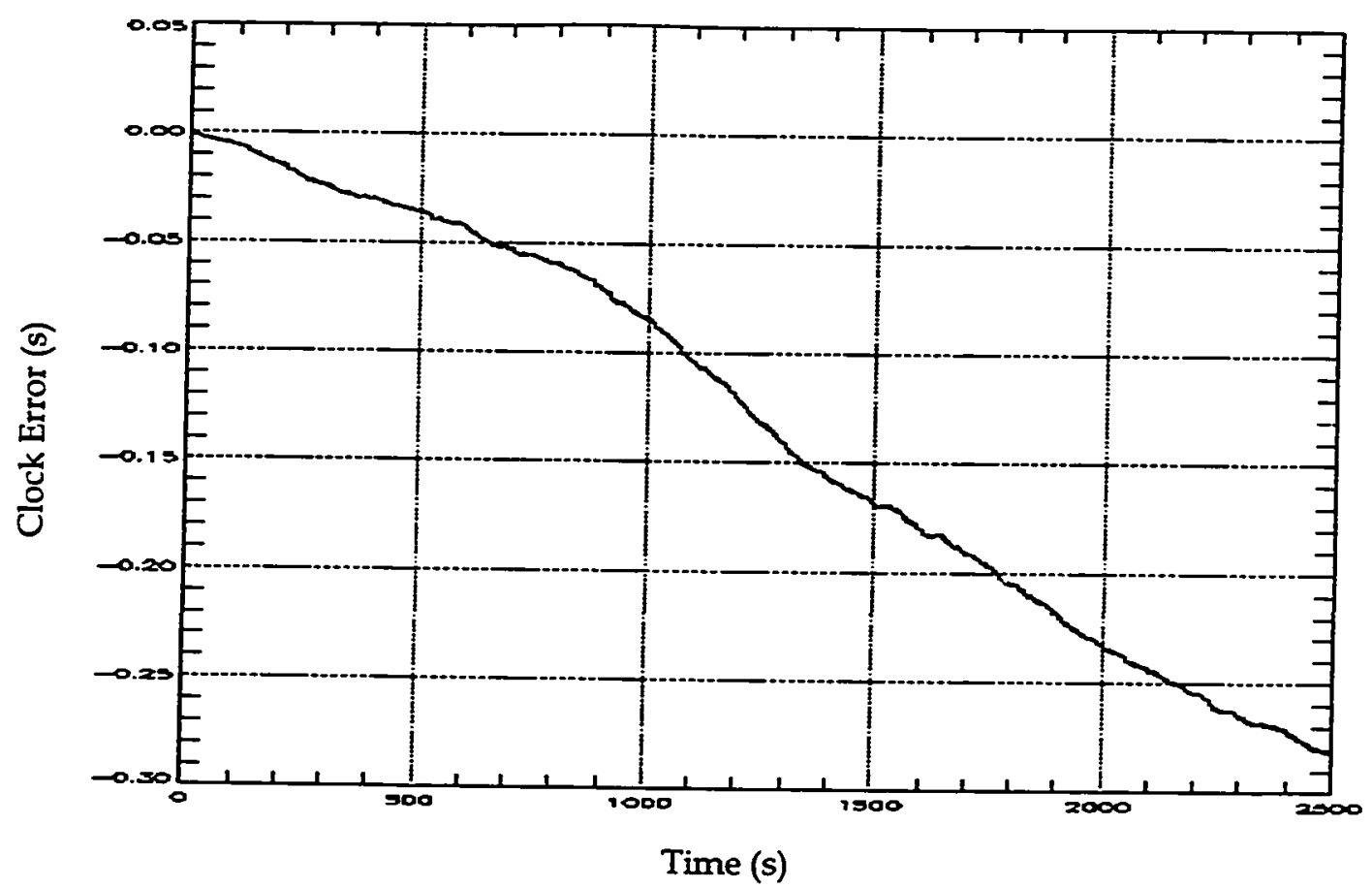

Figure 5.6: PLL Simulated Performance (Zero Pulsar Input)

This simulation has shown that it is possible to use a phase locked loop to track the pulsations of an $\mathrm{x}$-ray pulsar and use that signal as an autonomous time reference. While the performance of this loop is acceptable, its design is certainly not optimum and must be improved before use on the USA Experiment. 


\section{Chapter 6: Conclusions and Recommendations}

\subsection{Conclusions}

Emissions from stellar sources in the infrared, optical and ultraviolet wavelengths have been used as the basis for navigating satellites for more than three decades. Emissions in the $x$-ray regime have characteristics that suggest they also form an excellent basis for determining satellite attitude, time and position. X-ray navigation offers the potential for the autonomous determination of attitude, time and position from a single instrument operating in regimes from low earth orbit to geosynchronous orbit to interplanetary transfer to orbit about other planets. Furthermore, the x-ray regime offers the only fully autonomous measure of time on board a spacecraft.

The launch of the Unconventional Stellar Aspect Experiment in late 1996 will be the first opportunity tn demonstrate some of the principles of $x$-ray navigation.

This thesis serves as a case study for designing, manufacturing and testing experiments for space applications in small, semi-autonomous groups. This has been 
done through the structural and thermal design and analysis of the Unconventional Stellar Aspect Experiment.

Furthermore, this thesis is the first exploration of concepts of $x$-ray navigation. A variety of collimated $x$-ray instruments have been suggested for use on spinning spacecraft. The performance of a full attitude sensor with a single collimator has been demonstrated by using data from a previous spinning mission called HEAO-1. The improved performance of a dual collimator system was also demonstrated with this data. These instruments can provide attitude estimates with accuracies in the range of $0.01 \mathrm{deg}$. For more accurate attitude knowledge requirements on slowly spinning vehicles, an imaging $\mathrm{x}$-ray system was considered and limits on the bandwidth of measurements from such a system were estimated. It was shown that exposure times from $0.0008 \mathrm{sec}$ to $1 \mathrm{sec}$ would be required depending on the guide stars in the instrument field of view.

The potential for determining local spacecraft time autonomously was demonstrated by simulating the operation of a phase locked loop with input from the Crab Nebula pulsar. This pulsar has a period of 0.033 seconds and a complex pulse shape. The characteristics of the pulse shape and the Poisson noise associated with the background were included in the simulation. It was shown that a simple PLL design could attain frequency lock with the pulsar in about $500 \mathrm{sec}$. Lock was maintained with a steady-state error for the length of the simulation without any cycle slip and with a time error of about $0.0015 \mathrm{sec}$. 


\subsection{Recommendations for Future Research}

While many of the concepts of $x$-ray navigation have been demonstrated, there is still a great deal of work to be done before $x$-ray navigation is accepted as a standard method for determining the states of satellites. To this end the following areas of research are suggested to extend this concept:

1. While the USA mission is scheduled for launch in 1997, there is still much work to be done in support of its $\mathrm{x}$-ray navigation mission. Algorithms must be developed to determine the attitude of the spacecraft while using the USA instrument as a scanning collimator. This instrument can also be used to test the idea of identifying guide stars based on their pulsations. Flight code needs to be written for the RH32 to run the algorithms in real time.

Additionally, further simulations will be required to validate the PLL concept for the USA mission. These include simulations of other $x$-ray pulsars, the optimization of the PLL design for the USA case and the writing of flight code for the RH32 processor.

2. There is still a vast amount of data from the HEAO mission that can be used to prove the $\mathrm{x}$-ray navigation concept. The pointed mode high bit rate data can be used prove the concept of $x$-ray timing. There may also be high bit rate data of pulsars where HEAO is in a scanning mode. This data can be used to explore guide star identification concepts as well as $x$-ray timing on spinning vehicles. Furthermore, this database can be used to demonstrate that the differential x-ray star scanner can be used on guide stars whose intensities show significant temporal variability. 
3. This work has barely touched on the limits in accuracy that can be achieved by $x-$ ray star mapper systems. The design of coded masks for and deconvolution techniques for these systems have not been studied. Note that this problem is unique in that a priori information is available to the reconstruction algorithm. The problems associated with manufacturing, calibration and deployment of these instruments must also be addressed.

4. Finally, before these instruments can be used effectively and reliably, a number of design studies need to be performed. How small can we build these instruments? How large should the field of view be? How much power will they require? What are the ultimate performance limits in terms of satellite spin rate and jitter? 


\section{Appendix A: Unconventional Stellar Aspect Experiment Interface Control Document}




\section{P91-1/UNCONVENTIONAL STELLAR ASPECT (USA) \\ Interface Control Document} Security Manual. Section 11-19. or DOD Regulation 5200.1 AFR 250-1. Information Security Program. Chseter DX. For unclessified. limited documents. destroy by any mathod thet will prevert zisclasure of contents or reconeruction of the document.

WAFNONG

This documont contains technical date whoes export is cestricted by Ams Exoor Control Act TTin 20 US.C. 2751 of sea). or Exucutive Order 12470 or the Expon

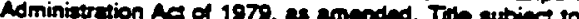

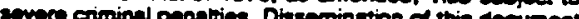
i comrolind under DOO Directive 5230.25.

Rockwoll intornational swoos entom avion Pogen

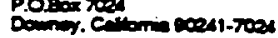




\subsection{SCOPE}

\subsection{SCOPE}

This Interface Control Document (ICD) defines and controls the design at the interface between the Unconventional Stellar Aspect (USA) Experiment and the P91-1 space vehicie (SV). Experiment ground support equipment (GSE) to Integrated Space Vehicle (ISV) GSE design interfaces are also defined. This ICD is intended to ensure compatibility between the USA and the SV by documenting form. fit, and functional interface agreements required to satisfy design and installation. Ground checkout and launch operations are documented in the Ground Operations Requirements Document (GORD). Launch facility to GSE interfaces are also defined in the GORD. Orbital operations requirements are defined in the Fight Operations
Requirements Document (FORD).

The interface requirements specified in this ICD take precedence over experiment requirements stated in the P91-1 contract.

\subsection{EXPERMMENT MISSION DEFINTIION}

The primary objective of the experiment is to test new approaches to the navigation of satellites. based on use of sensors operating in X-ray wavelengths observing celestial sources in those same bands. This calls for observing the brightest $X$-ray sources in the sty for prolonged periods and making a variety of measurements using $X$-ray sensors. Navigation of satellites involves being able to determine a state vector that includes: (1) position of the satellite relative to the earnh; (2) the time of the satellite; and (3) the satellite orientation. Different kinds of measurements support determination of these quantities. The measurements that are made sometimes characterize the sources themselves ( including characterizing physical mechanisms responsible for production of $\mathbf{X}$-ray emission) and other times directly lead to ascertaining one or more of the quantities comprising the state vector. To provide flexibility in scheduling observations and to give access to as many targets of opportunity as possible, it is necessary that the detectors have access to as large an amount of sky as possible. This coupled with the requirement to observe terrestrial sources translates into a wide range of pitch and yaw travel.

The USA experiment will observe sources for cumulative times of up to a month. but it switches from one source to another fairly frequently, typically twice per orbit. The observing program supports a number of different experiments and studies. Both the position and time determination tasks are carried out essentially by measuring $X$-ray count rates as a function of time and suitable processing the profiles so obtained. In the case of timekeeping. will calls for timing events to considerable precision, it is of central importance to be able to assign X-ray events an absolute time on the order of one microsecond. The celestial sources include precise natural clocks and it is important to time them precisely in order to exploit this feature. Some of these celestial events, such as eclipses. occur only occasionally and observations of them must be made at specific times. Examples of specific tasks include: (1) observation of horizon crossings of X-ray sources: and (2) observation of transitional events such as lunar occultations or eclipses or X-ray sources. particularly pulsars. In addition to the observation of celestial sources for navigation. there are some further tasks that involve observing terrestrial sources.

Examples of these terrestrial observations would include: (1) taking data at VLF ground stations simultaneously with USA observations of an X-ray source to correlate the variation in received VLF signal characteristics such as amplitude or phase with variations in $X$-ray input; (2) using those results to improve modeling of ionospheric response to ionization input; and (3) looking downward with the $X$-ray detectors to search for predicted $X$-ray flux associated with electrons precipitated by VLF signais. Downward-looking data are also used to investigate whether there are variations in $X$-ray flux from the earth at low latitudes that could serve as diagnostics for local enhancements of ionization or electron precipitation. 


\subsection{INTERFACE ITEM DESCRIPTION}

1.3.1 PQ1-1 Description. The major elements comprising the P91-1 space vehicle system (SVS)
are shown in Figure 1.

1.3.2 USA Experiment Definition. USA consists of two $X$-ray sensors and a gimballed (provided by the experimenter). Both sensors fit into a 2 -axis gimballed pointing assembly inputs from the $X$-ray sensors and is part of the cens navigation experiment computer processes pertectronics box that is mounted on USA.

The X-ray unit observes celestial sources and makes measurements that can be used for putonomous satellite navigation. The $X$-ray unit performs three navigation tasks (timekeeping, these simultaneousiy. The $X$-ray unit's determination), but does not necessarily do all three of ime-varying $X$-ray output of celestial sources which can of observation are: (a) measuring the imekeeping system; (b) of celestial sources which can be processed to provide an autonomous atmospheric system; (b) observing horizon crossings for autonomous position determination and soirces the sources through the field of view for aspect determination; and (d) observation of terrestrial $X$ emission used for any of these purated with very low frequency (VLF) transmitters. The X-ray assembly.

The X-ray sensor assembly consists of a pair of proportional counter units. These units are identical. Each $X$-ray detector includes a gas supply system to replenish lost detector gas. The collimators have fields of view that do not exceed $1.5^{\circ} \times 1.5^{\circ}$ (FWHM).

USA's on-board computer is a high performance autonomous navigation experiment computer. It has sufficient computing power to carty out a wide variety of navigational data analysis tasks using covered within the power on-board sensors as input. Power requirements for this computer new argin the power budget of the satellite. It is expected that from time to time that new algorithms will be uplinked and loaded in order to refine the navigational methodology. 


\subsection{USA/SPACE V'EHICLE INTERFACE REQUIREMENTS}

\subsection{GENERAL INTERI ACE REQLIREMIENTS}

The interface requirements specified in these paragraphs apply to USA and the space vehicle and include requirements imposed on the total P9! - I sp:ice syisten resulting from the mission profile.

\subsubsection{Onerational Orhit. The sun-synchronous orbit is as follows.}
Apogee:
Perigee:
$4.50 \pm 10 \mathrm{n} . \mathrm{mi}$
Inclination:
$4.50 \pm 10 \mathrm{n} . \mathrm{mi}$
Beta Angle:
$98.7 \pm 0.06=$ Period of Orbit: $\quad$ (OOL) betis angle of $15^{\circ} 1054^{\circ}$
$36 \pm 3^{\circ}$ beginning-of-life (BOL) with a drift potential to an end-of-life

NOTE: Beta angle is defined as the angle between the orbit plane and the geocentric radial sun position vector as shown in figure 3 . The beta angle is defined to be positive if SV motion is counterclockwise when seen from the sun.

3.1.2 On-Orbil Life. The mission supports experiment operations for a service life of one year afier space vehicle checkou: and test on-orbit. The ISV has an on-orbit design life of three
years.

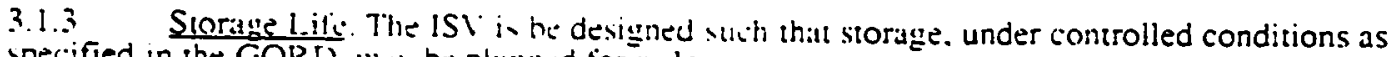
specified in the GORD. m.s be plamned for as lon! : ts tu'o yeurs.

3.1.4 Coerdinate Sistem. The coordinate system of the ISV is as shown in Figure 4. The

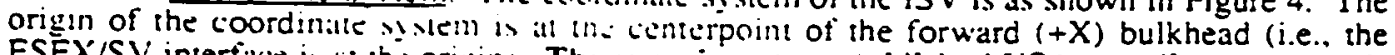
ESEX/SV interface is ai the orign, The expcrimenter established L'SA cuordinate system is shown in Figure?.

3.1.4.1 Earth Centered Inertial (ECl, coordinate Systen. The ECI coordinate system is defined such that the vectors originate at the earth's center. The $+Z$ vector passes through the north pole. the $+X$ vector passes through the vernal equinox (year 2000), and the $+Y$ vector is normal to both the $Z$ and $X$ vectors wing the right hand rule $(\hat{\mathbf{Y}}=\hat{Z} \times \hat{X})$. See Figure 5

\section{i.2 STRLCTLRE AND MECHA VIC.M! INTERFACES}

The interface requirements detine the form. fit. and access requirements for the installation of the experiment external and inte:nal to the space vehicle. Structure and mechanical interfaces will be as shown in Figure 6 . Sive and shape of the experiment is limited to the envelope shown in Figure 6.

\subsubsection{Loads}

3.2.1.1 Static Loads. The specified loads include the steady-state loads applied to the center of gravity of the experiment componenis. Forces and noments at the experiment/P91-1 interface are defined in Figure 7. Table 1 defines the design loads for the experiment given at the experiment c.g. A factor of sitiety of 2.0 for experiment primary structure is required if static load testing of the siructure is not to be pertormed. Mirgins gredier than zero are required. A fracture contrul anal sis per MII-STD-1522 "ill be performed by LSA for the pressure vessels.

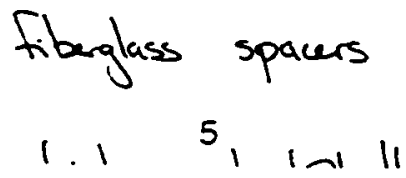


3.2.2 Stiffness Requirement. When the experiment is in its launch locked configuration with a rigid clamped interface, the fundamental natural frequency of the experiment is $\mathbf{5 0}$ $H \%$ or greater axial and lateral.

3.2.3 Eield-of-Regard and Field-of-View. The field-of-regard and field-of-view of the experiment are in accordince with Figure 8.

3.2.4 Mass Properties. Total weight of the experiment is limited to $\mathbf{4 5 3}$ pounds including growth margin. This weight excludes the SV mating electrical connectors and the mounting attach hardware supplied by the SV Contractor. Reference paragraph 3.5.3 for center of gravity (c.g.) and inertia limits for gimballing components.

Mass property reports are submitted with additional reports required if there are major changes to the weight, c.g.. or inertia data. Weight, c.g.'s and inertias are provided separately for gimballing components and other separately packaged components.

3.2.5 Mounting Provisions. Mounting provisions are as shown in Figure 6. Interfacing footprints, bolt locations and sizes are controlled to within \pm 0.005 inches. Interfacing drill remplates are designed and manufactured by Rockwell from dimensional drawings provided by the experimenter. The interfacing drill templates as provided to the experimenter.

3.2.5.1 Mounting Surfives Finish. Surface finish at each USA to ISV attachment interface is $\leq 4 \mathrm{R}$ microinches.

125

3.2.5.2 Mounting Surfice Flatness. Mouning: interface flatness is not applicable since alignment shims and thermal isolation are required at the USA to ISV interface. Shimming is to be provided as needed for installation.

\subsubsection{Bonding Straps. Bonding strap provisions are as defined in Figure 6.}

3.2.6 Exiernal Moving Parts. Moving parts are in accordance with Figure 8. The USA moving parts are the two gimballing sensors and the launch lock mechanisms.

3.2.7 Alignment. USA is aligned to the SV Inertial Reference Unit A (IRU-A) alignment cube.

3.2.7.1 Alignment Devices. The SV Contractor supplies the equipment and personnel needed to perform the alignment or the experiment to the SV. USA supplies a mirror cube that has been previousl: mounted and aligned b? L'SA un the USA detector boresight. The mirror cube will have $a$ clear line-of-sight frum the $+Y$ and $+Z$ sides of the $S V$ and will be securely mounted and protected so as to survive ISV level acoustic and thermal vacuum testing without loss of calibration to the boresight. The mirror cube, shown in Figure 6, may be removed at the experimenter's discretion, after final alignment verification.

3.2.7.2 Alienment Accuracy. USA is aligned to the SV IRU-A alignment cube to an alignment accuracy within 0.5 degrees.

3.2.7.3 Alignmens Knowledge. Alignment in 2 axes prior to shipment launch site is measured within an accuracy of one arc-minute with respect to the IRU-A alignment cube.

\subsubsection{Interface Materials}

3.2.8.1 Space Vehiclel SA Injerface. The SV/USA interfacing materials, mounting fasteners, and electrical bonding provisions are shown in Figure 6. 
3.5.4 Scan Profile. All USA pointer motions have the same general form, although the details of how much and how fast each axis moves depends on what task is being performed. The four general types of motion are track, slew, raster scan, and special. $A l l$ pointer velocity changes are made with an acceleration/deceleration of $2 \% \mathrm{~min} / \mathrm{sec}$ until the final angular velocity is reached. The acceleration maintains the disturbance torque about each axis below 2 oz,-in. The maximum rotation rate is $20 \% \mathrm{~min}$. One or two axes may be driven depending on the type of motion being performed. For the track mode the pitch drive accelerates to $4 \%$ min and remains at that value with minor deviations to correct for changes in the satellite attitude until the end of the observation, typically 15 to 20 min; after the observation the drive decelerates to zero velocity. During the typical observation the yaw drive operates only sporadically to correct for attitude errors. During a slew both drives accelerate to the maximum velocity of $20 \%$ min until the next target is reached. There is typically a rotation of $90^{\circ}$ to $120^{\circ}$ about the pitch axis. The motion about the yaw axis is determined by the relative positions of the two targets and is typically between $0^{\circ}$ and $90^{\circ}$. The raster scan mode has a pitch drive velucity of between $0^{\circ}$ and $4^{\circ} / \mathrm{min}$; the yaw drive accelerates to a maximum of approximately $10 \%$ min for total travel of approximately $10^{\circ}$ to $15^{\circ}$, then decelerates, reverses direction, and accelerates back to its previous rate of rotation in the opposite direction. It continues this procedure for the duration of the observation. The special observation modes are used to track vehicles or terrestrial targets and involve motions about one or both axes at rates less than $20 \% \mathrm{~min}$.

3.6

THERMAL CONTROL

The SV thermal comtro! subsysem is designed and tested per MIL-STD-1540B protoflight levels. Expermen:s thermal control subsistems are designed and tested (qualification and acceptance) to experiment specific requirements.

3.6.1 USA/Space Vehicle Thermal Intertice. The experiment/SV thermal interface schematic is presented in Figure 17. The parameters controlled by the experiment those controlled by the Space Vehicle as well as parameters affected by both experiment and SV, are identified in Figure 17. Thermal isolation interfacing hardware is shown in Figure 6.

3.6.2 Interface Temperature and Heat Flux Limits. The allowable temperature limits and heat transfer rates at the themal interfate during on orbit operation are per Table 2.

3.6.3 US.4 Allow:ahle Temperature Limits. The experiment component operating and nonoperating allowable temperatures are included in Table 2 . The pouer dissipation levels are as shown in the power profile (Figure 14 and in Section 3.3.2.1.1).

3.6.4 Thermal Control Coalings. Materia!s. Surfites Treatument and Restrictions. The selection and specification of the external coutings on the experiment and on the SV surfaces affecting USA thermal control is to be coordinated between the experiment and SV contractors.

All thermal control materials, i.e.. multilayer insulation. paints, tapes, adhesives, etc., are of flight proven quality with outgassing properties for weight loss and Collected Volatile Condensable Material (CVCM) compatible with the outgassing criteria as defined in the P91-1 Contamination Contro! Plan (Section 4.2.3).

USA multilayer insulation ( $\mathrm{ML}$ ). heaters and space radiators are provided and installed on the instrument by the experimenter prior to delivery to the SV contracior for integration with the SV. 
No electroexplosive devices are used by the USA, however paraffin actuators are used to release the 2 axis gimbal and are controlled by USA via the 1553 bus. The release is fail safe. requiring
2 commands for release.

\subsection{SAFETY REQUIREMENTS}

The experiment meets the requirements of WSMCR 127-1, CSTCR 127-1 and the P91-1 System Safety Program Plan when the experiment is attached to the SV. The experimenter is required to support the SV contractual safety reviews. Experinent inputs to the P91-1 Hazard Analysis Reports are defined in Table 6.

The Certification of Safety is included in the Cerificate of Compliance (Table 4). The launch site or factory safery training requirements which are applicable to the experimenters are specified in the GORD.

A Failure Mode Effects Analysis (FMEA) of the experiment interface is required. The purpose of the FMEA is to verify that failure of an experiment does not cause failure of the $\mathrm{SV}$ including the 1553 interface bus, or another experiment. The failure effects analyzed are limited to the SV to experiment interface. MIL-STD-1629, Task 101, provides guidance
for this task.

3.8.1 Design Criteria. The experiment is designed to preclude any operation, event. condition, or failure that could cause detectable or undetectable malfunctions or damage to Py 1-1. damage to other equipment. injury: to personnel, or damage to facilities.

$$
\text { FLIGHT ENVIRONMENT }
$$

The following parameters represent the induced night environments to which the interfacing experiment is exposed during ascent and earth orbit. The experiment is expected to survive and/or operate when exposed to any feasible combination of those parameters encountered from ascent through mission operstion.

3.9.1 Acoussic. Maxinum predicted flight acoustic environment is shown in Figure 18.

3.9.2 Vibration. Predicted random and sinusoidal flight vibration levels for the space vehicle side of the USA interface are as show $n$ in Figure 19

3.9.3 Shock. The predicted piro shock levels at the SV/experiment interface are expected to be within the envelope as shoun in Flgure 20

3.9.4 Depressurizition and Repressurization. The payload fairing (PLF) gaseous environment consists of residue remaining from the round purge conducted prior to lift-off. The nominal PLF pressure during ascent is shown in Figure 21. 3.9.5 Effeci of External Magnetic Fields. No instrument sensitivity to magnetic fields of up
to 2 Gauss have been identified.

3.9.6 Badiation Environment. The total radiarion dose for the three year mission is about $1.5 \times 10^{4}$ rads behind nominal ( $100 \mathrm{mil}$ aluminum) shielding. Figures 22 and 23 provide the total dose and single event radiation environments.

3.9.7 Eree Molecular Heating. Free molecular heating at fairing sepatration is less than 0.1 BTL/fi2-sec. The free molecular heaning rate hislory is shoun in Figure 24. 
3.10

Test Environment. The ISV is subjected to those tests described in the GORD. Tests are to be conducted to the protoflight levels as defined in MIL-STD-154().

3.1 Elight Contanjination Linits. The experimenter controls their flight contamination below the level budgeted in the P91-1 Contamination Control Plan. Section 4.1. The experimenter controls and directs venting from experiment enclosure to prevent impingement of outgassed products on any ISV sensitive surface. 


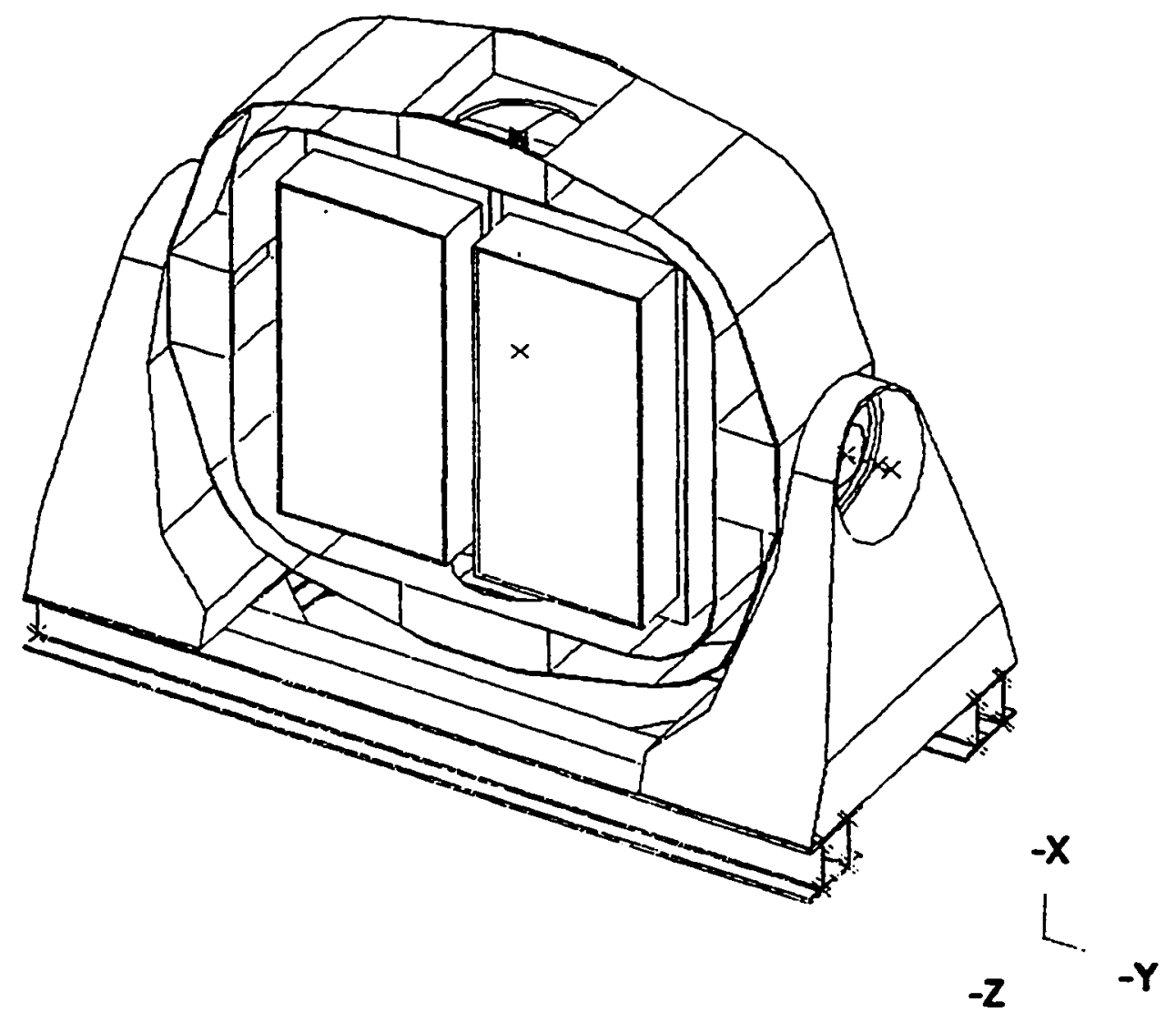

Figure 2. USA Experiment 


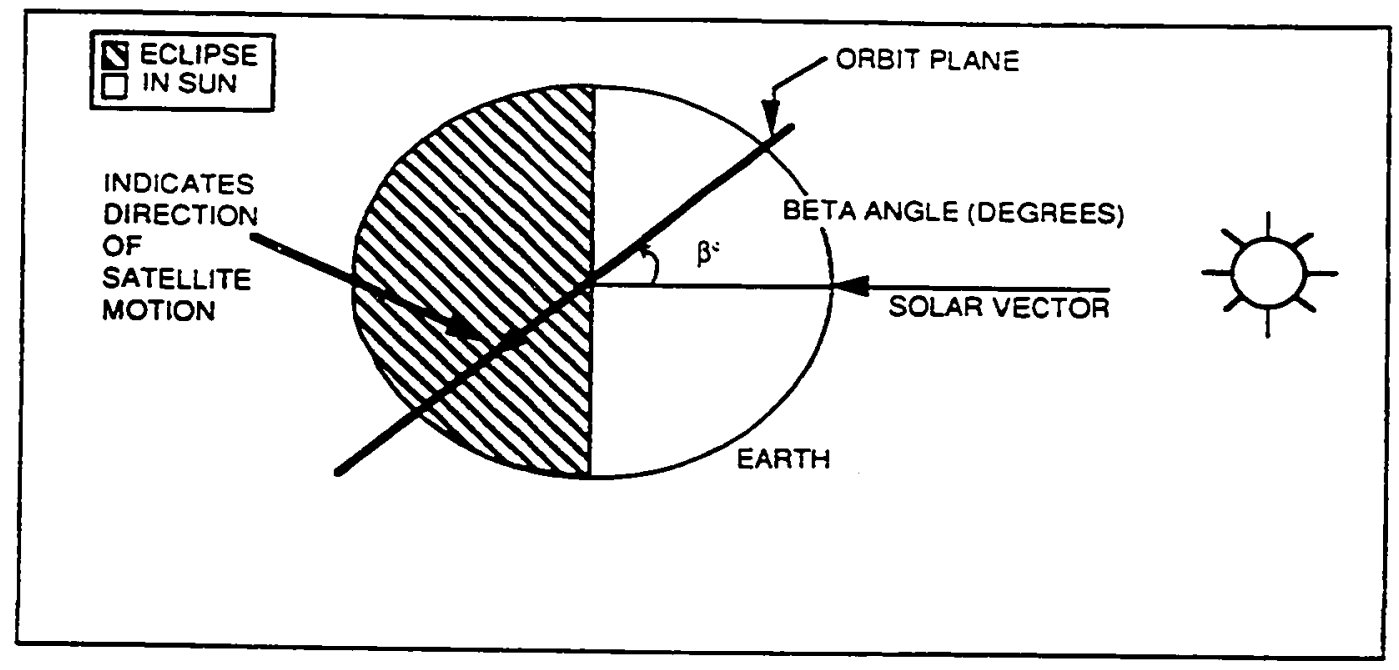

Figurc i. Bera Ans/c IIlasaration

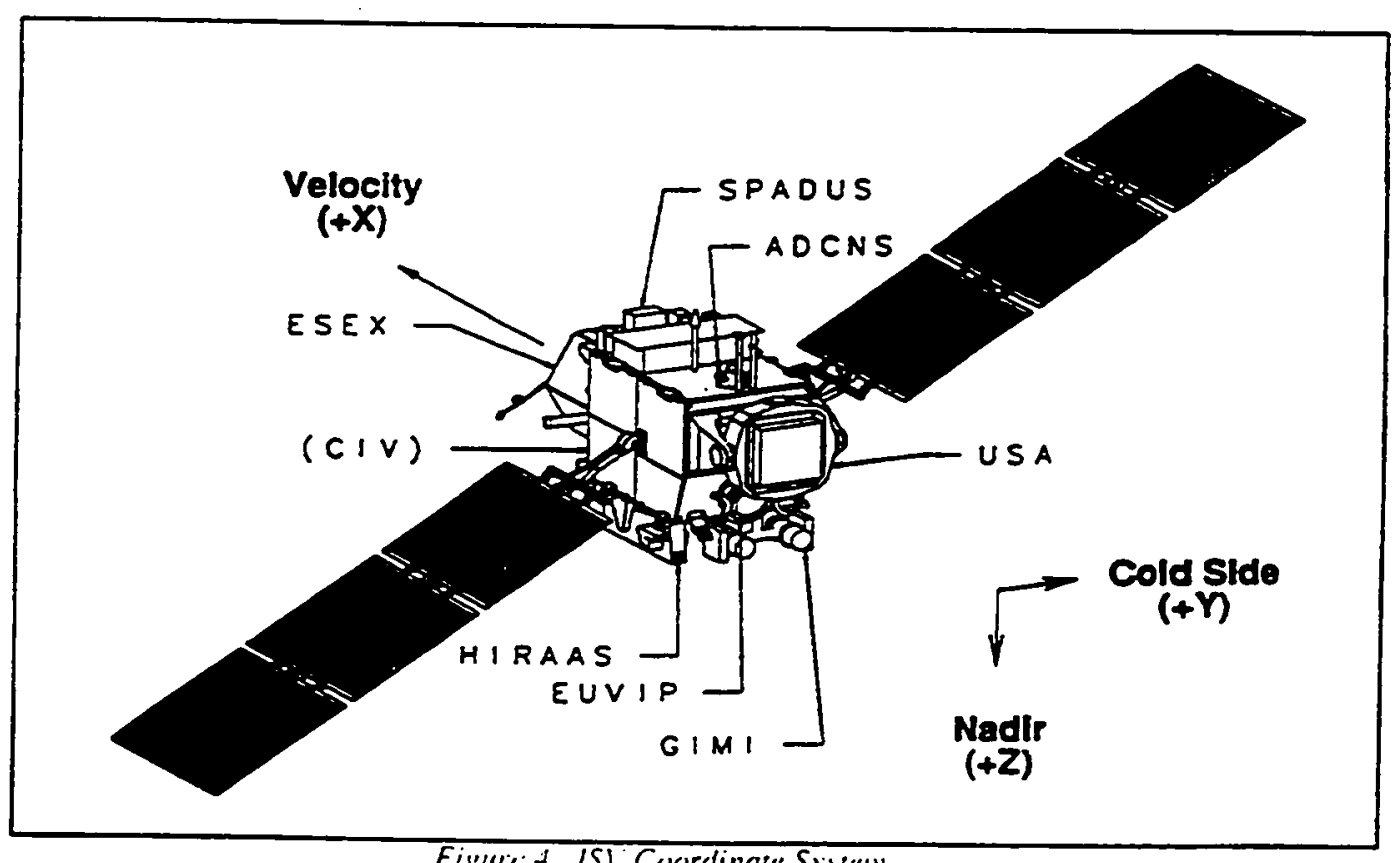

Fibure + ISI Coordinate Sistem 


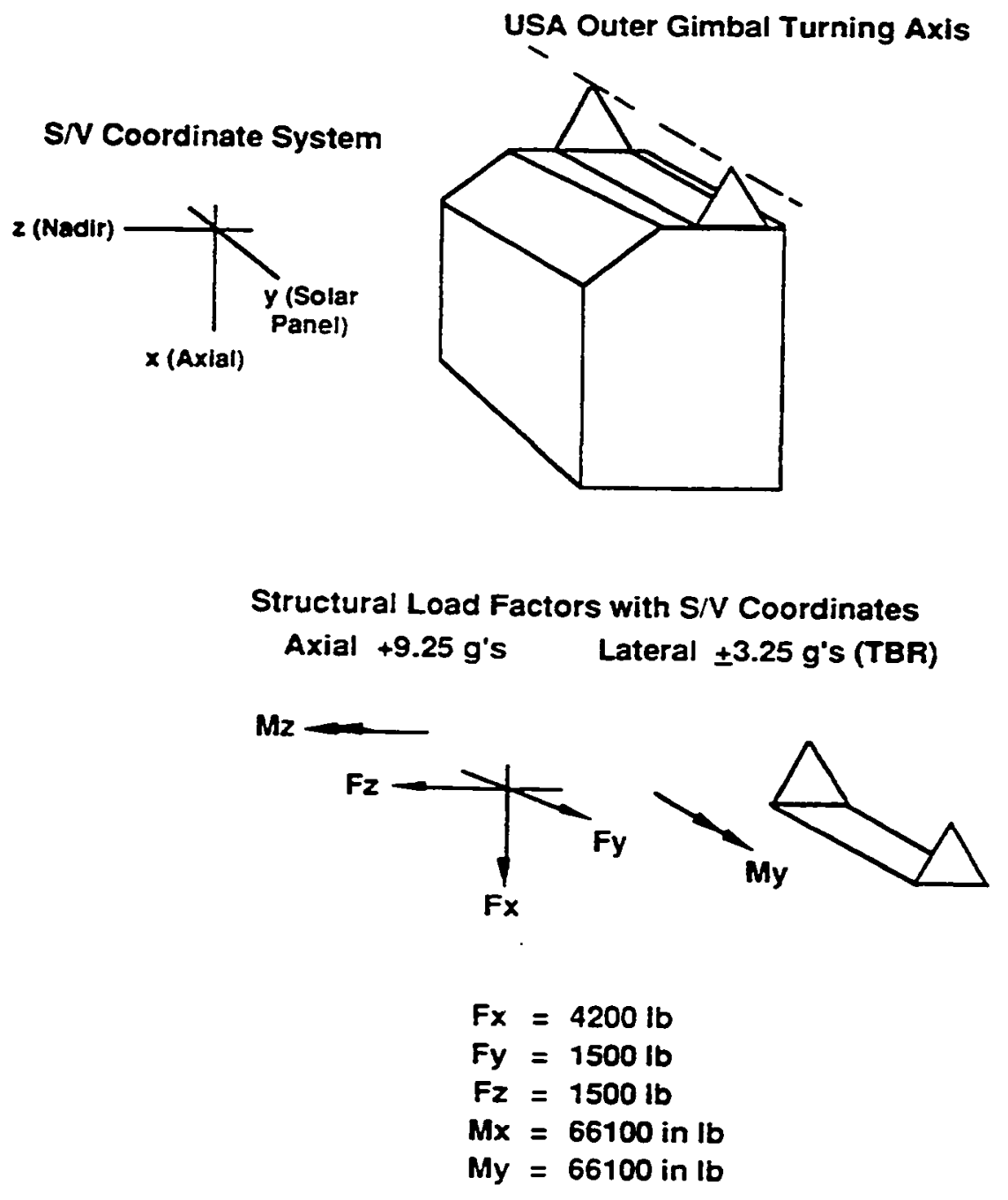

Figure 7. Interface Forces \& Moments 

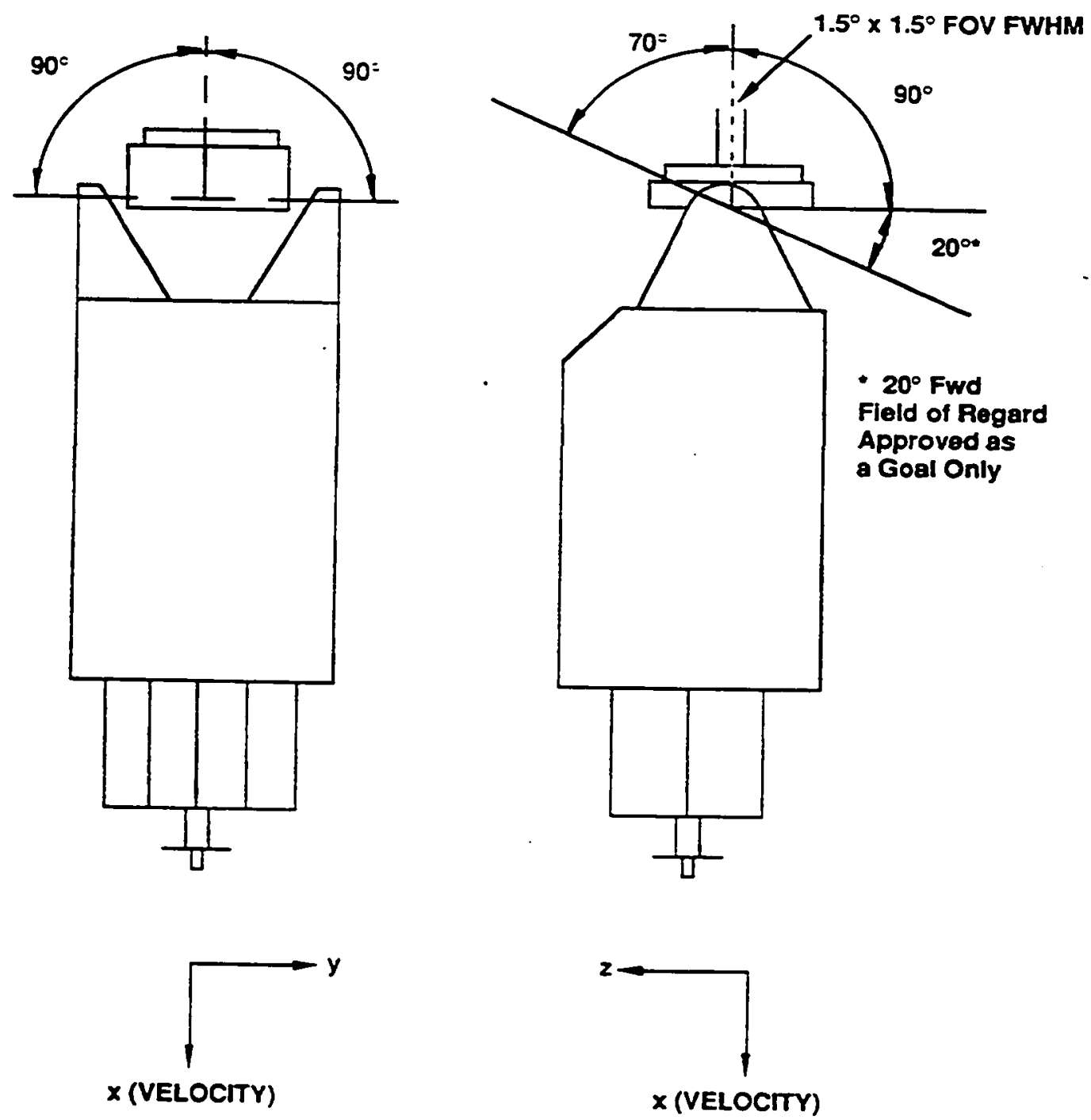

Figure S. LSA Field-of-Regard 


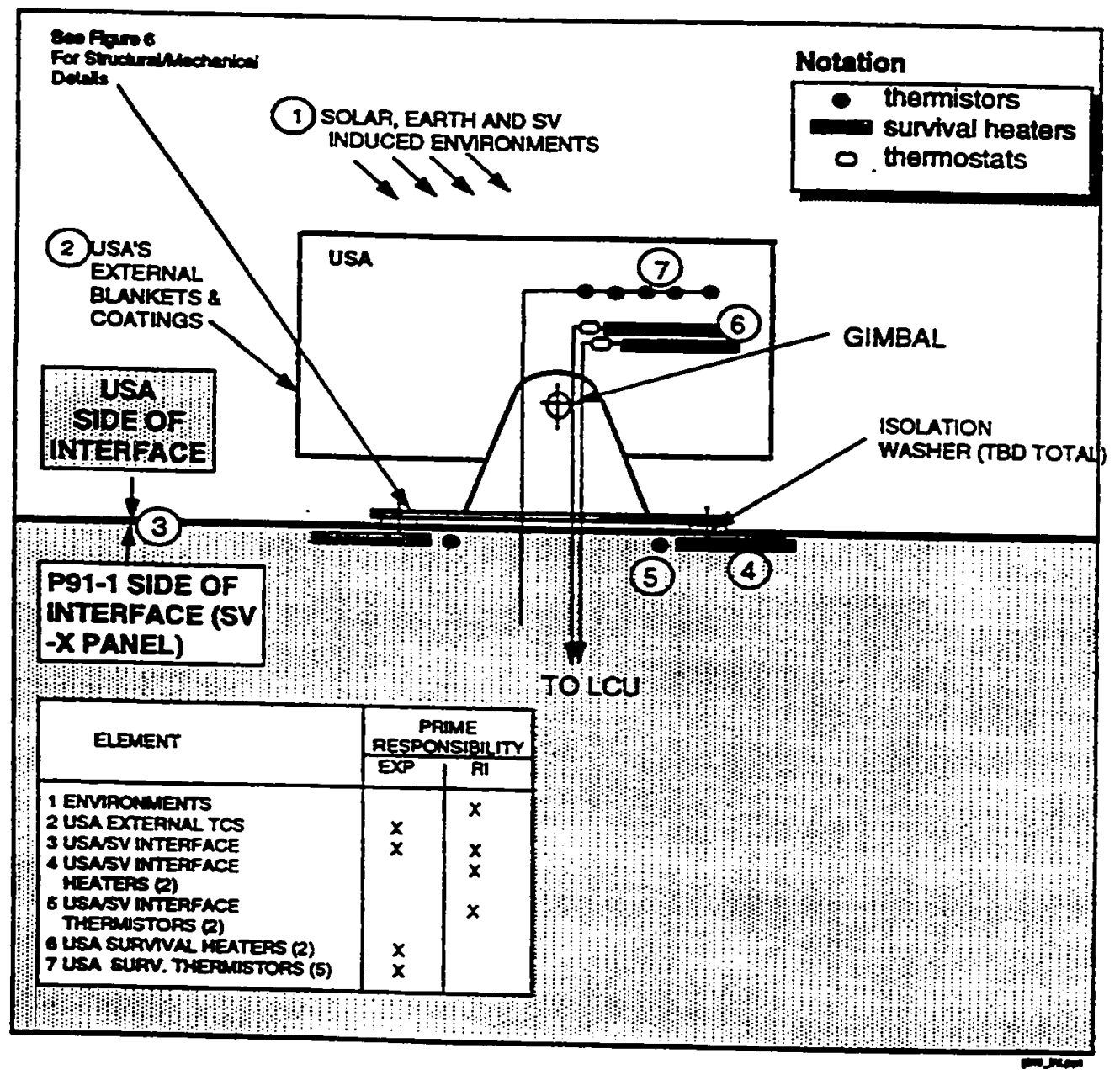

Fixure 17. USA SI Thermal Interfuces 


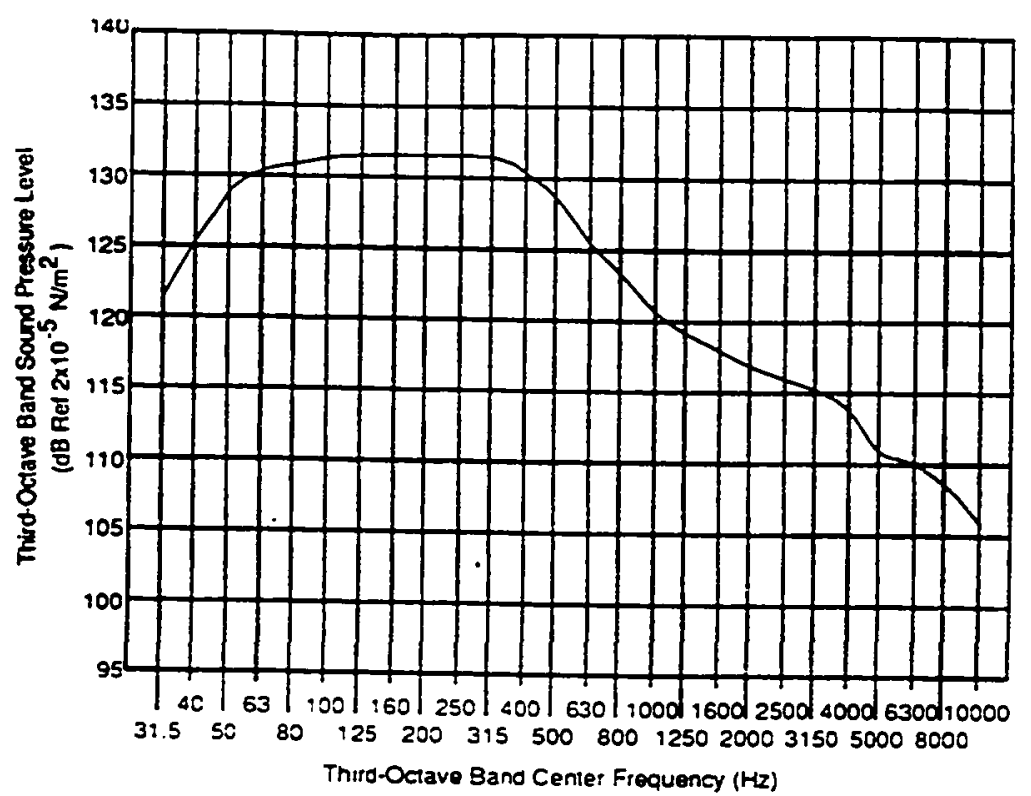

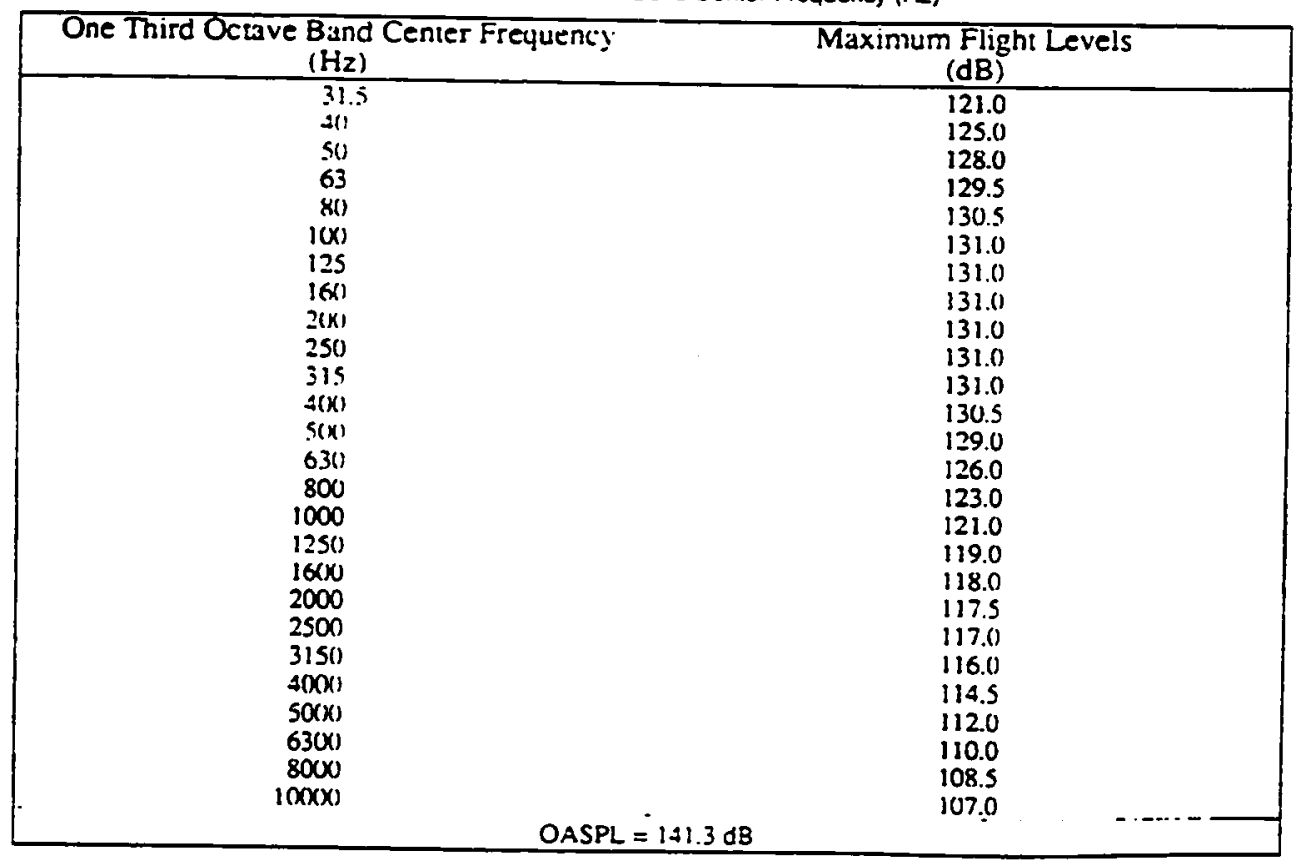

Figure 18. Acoustic Spectro 


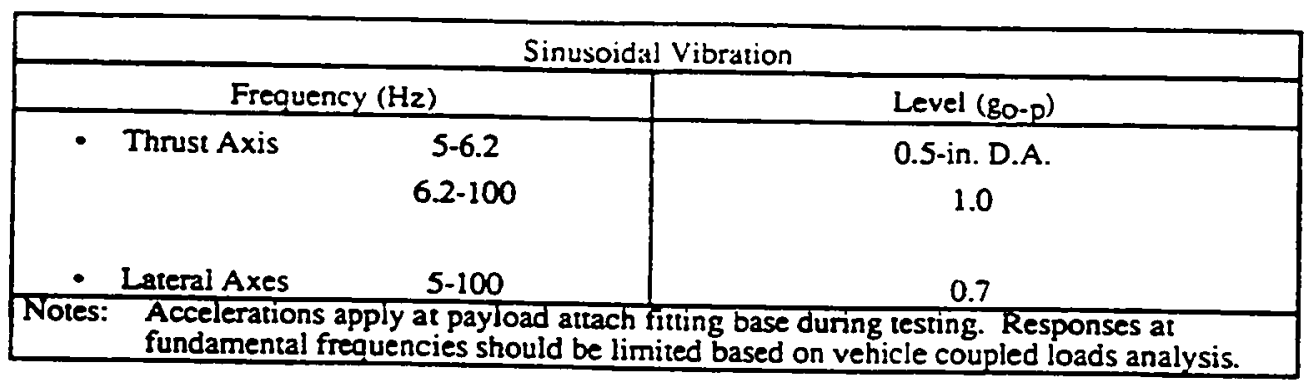

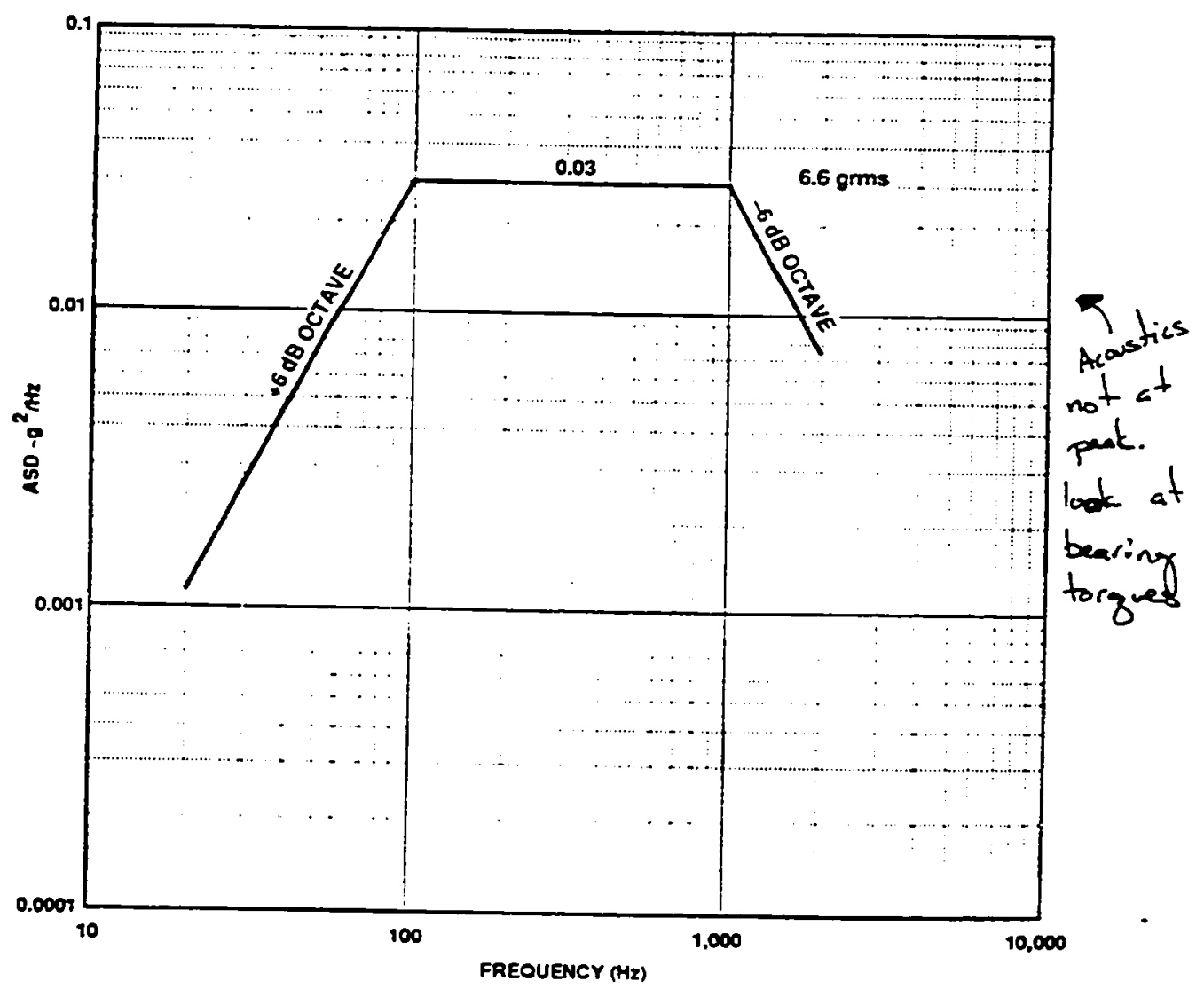

Figure 19. Maximum Predicted Flight Vibration Levels 


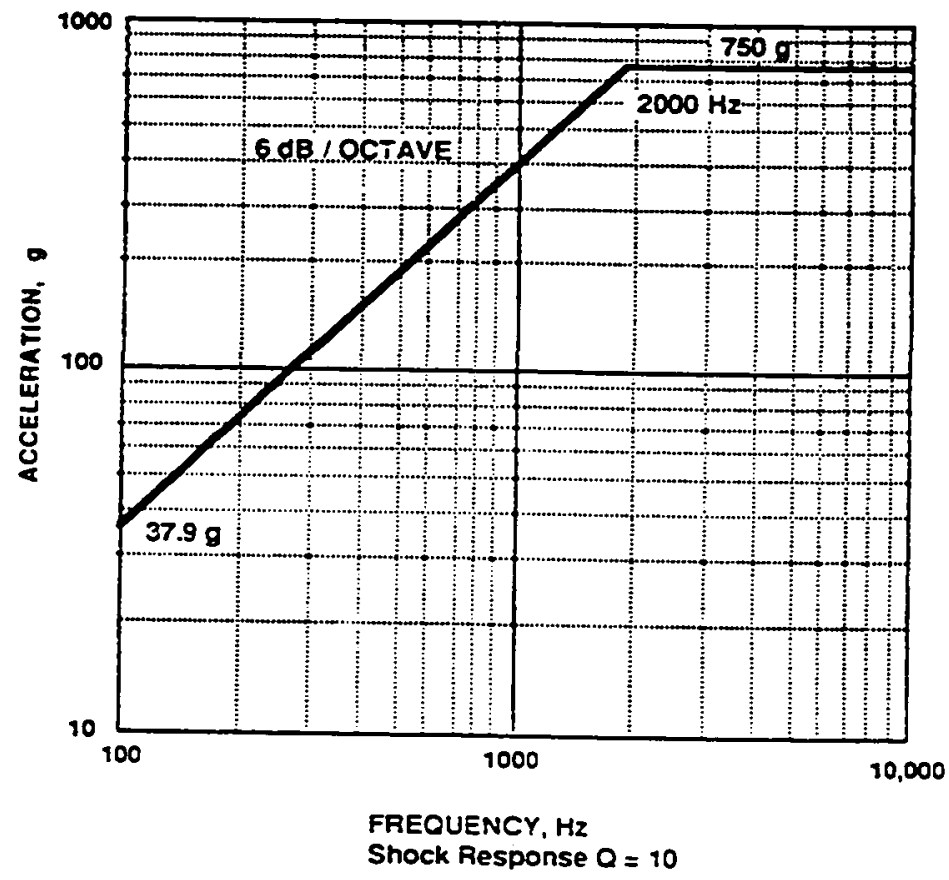

Figure 20. Predicted Pyro Shuck Level at USAISV Interface 


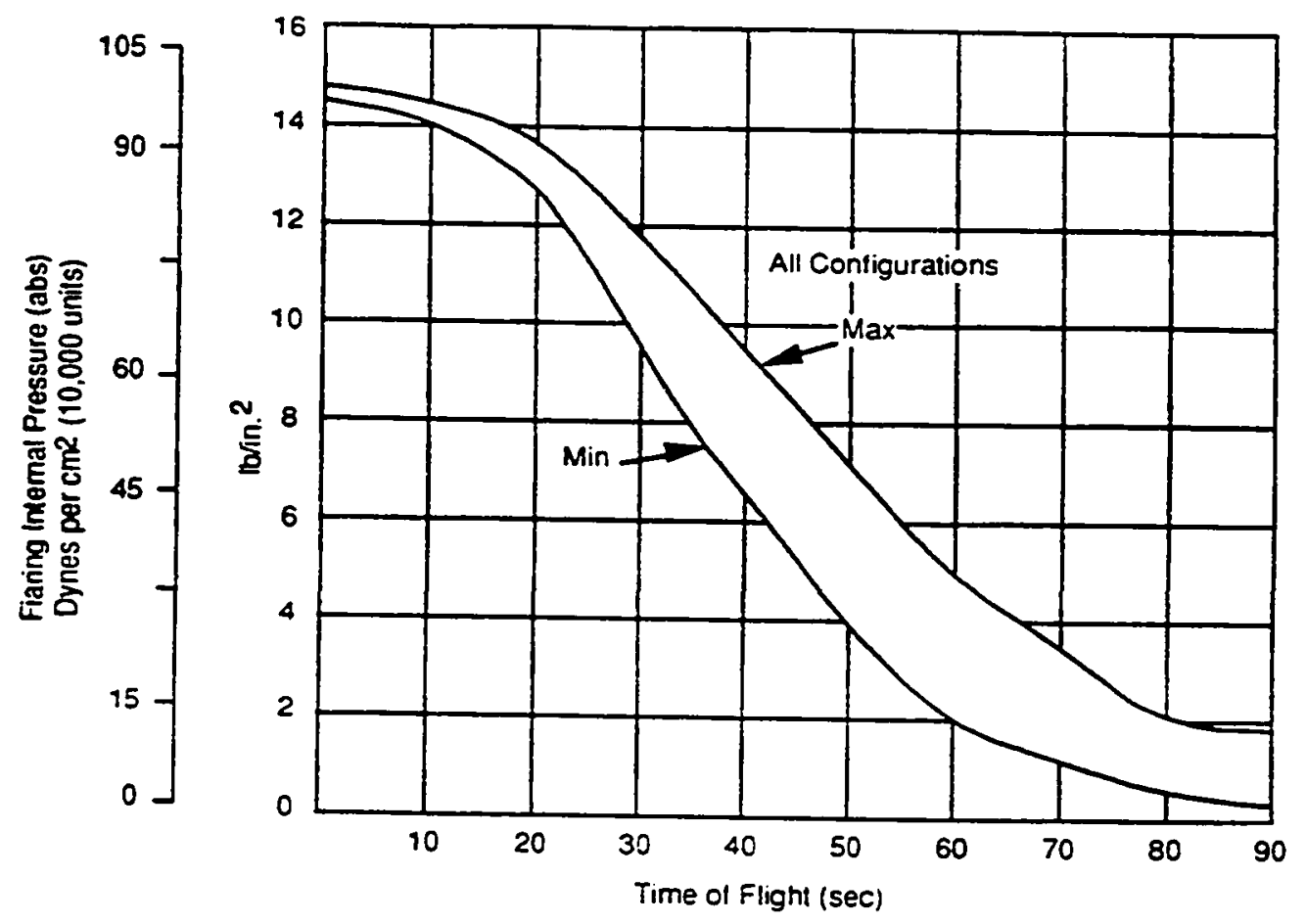

Maximum Rate of Change - 0.5 PSUSEC

Maximum Rate Over a 5 SEC Interval: 0.4 PSISSEC

\begin{tabular}{|c|c|c|}
\hline $\begin{array}{c}\text { Time of } \\
\text { Flight } \\
\text { (sec) }\end{array}$ & $\begin{array}{c}\text { Min } \\
\text { Pressure } \\
\text { (psi) }\end{array}$ & $\begin{array}{c}\text { Max. } \\
\text { Pressure } \\
\text { (psi) }\end{array}$ \\
\hline 0 & 14.5 & 15.0 \\
10 & 14.1 & 14.6 \\
20 & 12.7 & 14.3 \\
25 & 11.4 & 13.8 \\
30 & 98 & 13.4 \\
35 & 7.9 & 12.8 \\
40 & 6.4 & 12.2 \\
45 & 5.1 & 11.5 \\
50 & 3.9 & 10.7 \\
55 & 3.0 & 9.8 \\
60 & 2.2 & 8.9 \\
70 & 1.1 & 7.1 \\
80 & 0.6 & 5.2 \\
90 & 0.4 & 3.4 \\
\hline
\end{tabular}

Figure 21. Pressurc Levels Decas 
Table 1. Design Limit Flight Accelerations, $g(s)$

\begin{tabular}{|c|c|c|c|}
\hline & Axial & Lateral & \\
\hline -ift Off & & \pm 3.25 (TBR) & $=4.8$ \\
\hline Main Engine Cut Off & & \pm .13 & \\
\hline
\end{tabular}

Note: (-) Tension (+) Compression

imit loads include dynamic amplification of the ISV structure.

Table 2. USA/SV Interface Component Allowable Temperatures and Interface Heat Flow Limits

\begin{tabular}{|c|c|c|c|c|c|c|c|c|c|c|}
\hline \multirow[b]{2}{*}{ COMPONENT } & \multicolumn{3}{|c|}{ OPERATIONAL } & \multicolumn{3}{|c|}{ SURVIVAL } & \multicolumn{2}{|c|}{ ACCEPTANCE } & \multicolumn{2}{|c|}{ PROTOFLIGHT } \\
\hline & $\begin{array}{l}\mathrm{MIN} \\
(\mathrm{C})\end{array}$ & $\begin{array}{c}\text { MAX } \\
\text { (C) }\end{array}$ & $\begin{array}{c}\text { HEAT } \\
\text { FOW } \\
(W) \\
\end{array}$ & $\begin{array}{l}\text { MIN } \\
\text { (C) }\end{array}$ & $\begin{array}{c}\text { MAX } \\
\text { (C) }\end{array}$ & $\begin{array}{c}\text { HEAT } \\
\text { FLOW } \\
\text { (W) }\end{array}$ & $\begin{array}{l}\text { MLN } \\
\text { (C) }\end{array}$ & $\begin{array}{c}\operatorname{MAX} \\
\text { (C) }\end{array}$ & $\begin{array}{l}\mathrm{MIN} \\
\text { (C) }\end{array}$ & $\begin{array}{c}\text { MAX } \\
\text { (C) }\end{array}$ \\
\hline USAVSV Interface & -20 & 40 & $13^{* *}$ & -20 & 40 & -12 & N/A & $N / A$ & N/A & N/A \\
\hline \multicolumn{11}{|c|}{ The following temperatures are listed for Reference Onls; e.g.. thermal vacuum test } \\
\hline Electronics & -13 & 50 & - & .29 & 66 & 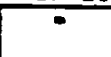 & .24 & 61 & -29 & 66 \\
\hline $\begin{array}{l}\text { Detectors/Viton } \\
\text { Scals }\end{array}$ & 0 & 50 & - & -15 & 66 & - & -10 & 61 & -15 & 66 \\
\hline
\end{tabular}

-See Section 3.3.2.1.1 for Power Dissipation

** $(+)$ Heat Flow is to SV; (-) Heat Flow is to Experiment

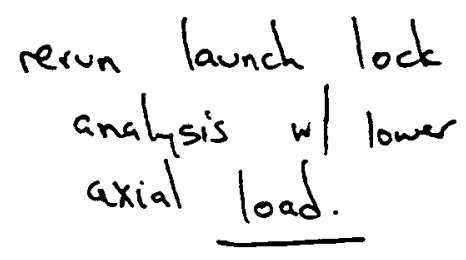




\section{Appendix B: Selected Astrogravity Notes}




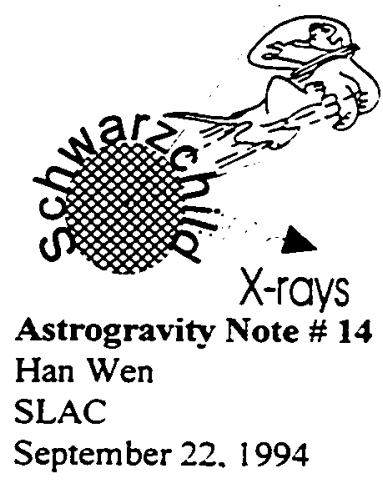

\section{Determining Dead Time}

We derive a simple analytic expression for the dead time' ${ }^{\prime}$ and verify its form with a Monte Carlo simulation. The only assumptions we make is that the distribution of counts follows Poisson statistics and that the dead times are nonparalyzable.

We may relate the count rate, $\mathcal{N}($ to the time interval probability density function, $f(T)$ in the following manner.

$$
\frac{1}{\mathcal{N}}=\langle T\rangle=\int_{0}^{\infty} T f(T) d T
$$

where $T$ is a time interval. Assuming Possion statistics,

$$
P_{P}(n, \mathcal{N} T)=\frac{(\mathcal{N} T)^{n} e^{-\hat{N} T}}{n !}
$$

we may determine $f(T)$ by considering the probability of having a time interval $T \leq T$.

$$
\begin{gathered}
P\left(T^{\prime} \leq T\right)=\sum_{n=1}^{\infty} P_{P}(n, \mathcal{N} T) \\
=1-P_{P}(0, \mathcal{N} T) \\
f(T)=\frac{d P\left(T^{\prime} \leq T\right)}{d T} \\
\Rightarrow f(T)=\mathcal{N} e^{-\mathcal{N} T}
\end{gathered}
$$

'Muller. NIM 112 (1973) pgs. 47-57 
If we turn on a dead time. $\tau$ to determine the obsenved count rate, $\chi_{6}$ using eq. (1) we need to transform $f(T)$ to the observed time interval density, $f_{O}(T)$. We expect to $f_{O}(T)$ take the form.

$$
\begin{aligned}
& f_{o}(T) \begin{cases}=0, & T<\tau \\
\propto f(T), & T \geq \tau\end{cases} \\
& \Rightarrow f_{0}(T) \propto U(T-\tau) f(T)
\end{aligned}
$$

where $U(T-\tau)$ is unit-step function. Requiring that the integral of $f_{o}(T)$ be normalized to one determines the normalization to be:

$$
\begin{aligned}
& f_{o}(T)=U(T-\tau) f(T-\tau) \\
\Rightarrow & f_{o}(T)=U(T-\tau) \mathcal{N}\left(e^{-\mathcal{N}(T-\tau)}\right.
\end{aligned}
$$

Plugging this into eq. (1) gives.

$$
\begin{gathered}
\frac{1}{\mathcal{N}_{0}}=\int_{0}^{\infty} T f_{0}(T) d T \\
=\frac{e^{\mathfrak{N} T}}{\mathcal{N}} \int_{\mathfrak{N} T}^{\infty} x e^{-x} d x \\
=\frac{1+\mathcal{N} T}{\mathcal{N}} \\
\Rightarrow \frac{\mathcal{N}}{\mathcal{N}_{0}=\frac{\mathcal{N}}{1+\mathcal{N} T}, \quad f_{\text {dead }}=\frac{\mathcal{N}-\mathcal{N}=\frac{\mathcal{N}_{0}}{1-\mathcal{N}_{0} T}}{\mathcal{N}}=\mathcal{N} \mathbb{N}_{0}}
\end{gathered}
$$

We verified the function form of the fractional dead time, eq. (6) by performing a Monte Cario simulation of a Poisson process using a set dead time. Using the Poisson time interval probability distribution. eq. ( 3 ) we generated a series of relative separation times for a given count rate. $\{$. The distribution of these relative separation times, namely, $f(T)$ is shown in Fig. 1.

By summing the counts which did not fall within the dead time of previous counts we determine the observed count rate. $\chi_{6}$ for this simulation. Plots of the resulting fractional dead times vs. $\chi_{6}$ for two dead times, $11 \mu \mathrm{s}$ and $40 \mu \mathrm{s}$ are shown in Figs. 2-3. Both plots show a linear relationship between the fractional dead time and $x_{6}$ with fitted slopes agreeing with eq. 6. namely, equalling their set dead times. respectively. 


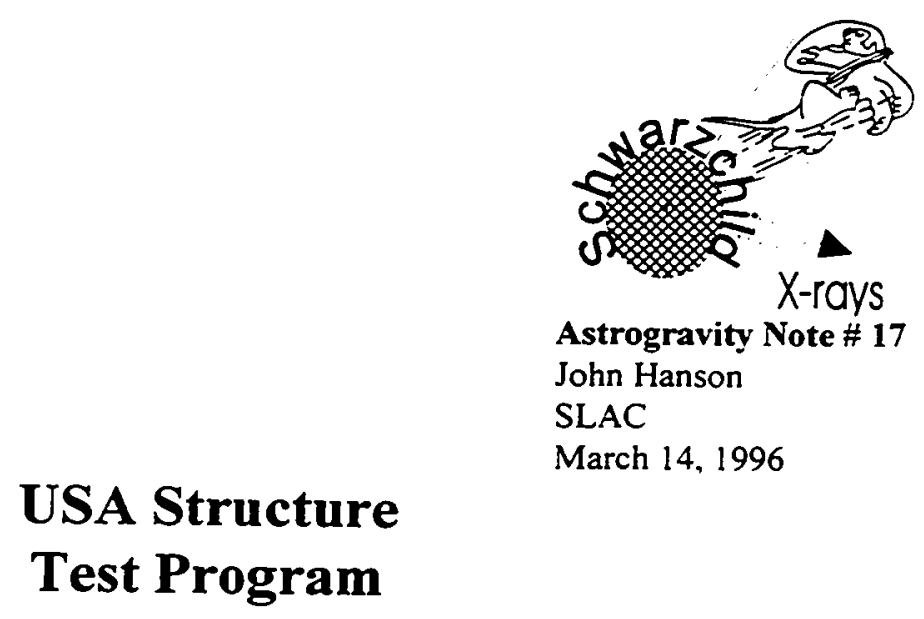

\section{Introduction}

In support of the Unconventional Stellar Aspect Experiment (USA), SLAC has designed. manufactured, and tested the gimbal support structure for the experiment. Constructed from 6061/T6 aluminum. this structure was assembled using a process called dip-brazing. An integral part of this effort was the test program that occurred simultaneously with the design and manufacturing processes.

\section{Test Program}

The concurrent design. manufacturing and testing program that was applied to the USA experiment is summarized below:

1. Finite element modelling:

The USA structure was modelled with the CAEDS/DEAS software package published by IBM and SDRC. The work will be described in a subsequent AstroGravity note.

2. Construction to established specifications:

All dip-brazing was done by Aluminum Dip Braze Co. (ADB) in Burbank. Ca. This work was done in accordance with Mil-B-7553-B Type V Grade B with quality standards per Mil-I-45208. All joint preparation was done to Mil-B-7553-B Type V Grade A specifications. Following the dip-brazing process, all parts were heat-treated to attain T6 conditions. All dip-brazed joints were then inspected radiographically and the results interpreted to determine the fractional braze wetting of each joint. Certifications of Conformance are on file with radiographs for all parts.

3. Manufacturing qualification: 
Because the dip-brazing process is relatively new to the aerospace community, a series of qualification test samples were built by ADB and tested to failure at SLAC. This work is described in detail in AstroGravity Note \#18.

\section{Prototype pylon and flight articles:}

After qualifying the manufacturing process, one prototype pylon was manufactured by ADB to prove the overall manufacturing concept. This pylon would later be subjected to a static load test. Only after visual and radiographic inspections of the prototype pylon were performed were the flight articles manufactured. The flight articles included two pylons and one yoke.

5. Quality assurance pull samples:

Each item dip-brazed by ADB was accompanied by five shear samples similar to those used in the manufacturing qualification process. These samples were prepared in the same manner and to the same specification as the prototype and flight articles and were brazed in the same bath and at the same time as their parent articles. The results of these tests are described in AstroGravity Note \#19.

\section{Static Load Test}

As a final test of the USA structure. the prototype pylon was used in a static load test that subjected the pylon to $115 \%$ of the USA ICD loads. This test is described in detail in AstroGravity Note $\# 20$.

\section{Test Summary} (Table 1).

The results of all facets of the USA structural test program are summarized below

\begin{tabular}{|c|c|c|c|c|}
\hline Test & $\begin{array}{c}\text { Pylon 1 } \\
\text { (Prototype) }\end{array}$ & $\begin{array}{l}\text { Pylon 2 } \\
\text { (Flight) }\end{array}$ & $\begin{array}{l}\text { Pylon } 3 \\
\text { (Flight) }\end{array}$ & $\begin{array}{c}\text { Yoke } \\
\text { (Flight) }\end{array}$ \\
\hline $\begin{array}{l}\text { Manufacturing } \\
\text { Qualification } \\
\text { Pull Samples } \\
\end{array}$ & $\begin{array}{c}6892 \text { psi } \\
\text { Mean Shear } \\
\text { Strength }\end{array}$ & $\begin{array}{c}6892 \text { psi } \\
\text { Mean Shear } \\
\text { Strength }\end{array}$ & $\begin{array}{c}6892 \text { psi } \\
\text { Mean Shear } \\
\text { Strength }\end{array}$ & $\begin{array}{c}6892 \text { psi } \\
\text { Mean Shear } \\
\text { Strength }\end{array}$ \\
\hline $\begin{array}{l}\text { Certification of } \\
\text { Conformance }\end{array}$ & On File & On File & On File & On File \\
\hline $\begin{array}{l}\text { Radiographic } \\
\text { Inspection }\end{array}$ & $\begin{array}{l}70 \% \text { min } \\
75 \% \text { avg }\end{array}$ & $\begin{array}{l}70 \% \mathrm{~min} \\
85 \% \text { avg }\end{array}$ & $\begin{array}{l}70 \% \text { min } \\
85 \% \text { avg }\end{array}$ & $\begin{array}{l}60 \% \mathrm{~min} \\
70 \% \text { avg }\end{array}$ \\
\hline $\begin{array}{l}\text { Quality } \\
\text { Assurance } \\
\text { Pull Samples }\end{array}$ & $\begin{array}{c}6973 \text { psi } \\
\text { Mean Shear } \\
\text { Strength }\end{array}$ & $\begin{array}{c}6491 \text { psi } \\
\text { Mean Shear } \\
\text { Strength }\end{array}$ & $\begin{array}{l}6341 \text { psi } \\
\text { Mean Shear } \\
\text { Strength }\end{array}$ & $\begin{array}{c}6037 \text { psi } \\
\text { Mean Shear } \\
\text { Strength }\end{array}$ \\
\hline Static Load Test & Passed & N/A & N/A & N/A \\
\hline
\end{tabular}

Table 1: USA Test Program Summary 


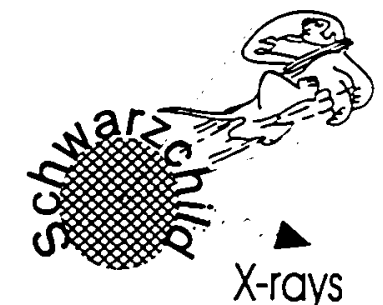

Astrogravity Note \# 18

John Hanson

SLAC

October 10, 1994

\section{USA Dip Brazing Manufacturing Qualification}

\section{Introduction}

The primary support structure for the Unconventional Stellar Aspect Experiment makes extensive use of a bonding process known as dip-brazing in its manufacturing. Two pylons and one yoke are to be assembled from components made of 6061/T6 aluminum that are dip-brazed together. In order to ensure that dip-brazed joints will have sufficient strength to survive the launch environment. a number of qualification braze joints were tested.

\section{Test Samples}

Two types of joints were tested: eight shear samples and eight tensile samples. Figures 1 and 2 show the dimensions of the shear and tensile samples. respectively. All samples were made from 6061/T6 aluminum. The test samples were dip-brazed by the Aluminum Dip Braze Co. of Burbank. Ca. All manufacturing was done to Mil-B-7883-B Type V Grade B with joint preparation to Mil-B-7883-B Type V Grade A. The samples were then machined to the final configuration shown in Figure 3.

\section{Test Results}

All samples were tested to failure using a tensile test machine in the Material Science Department of Stanford University. All test sample failure modes were brittle failure of the braze joint. The resulting failure loads are summarized in Table 1.

The strength of the braze joints are sufficient for the USA experiment, with mean joint failure stresses of 6892 psi for the shear samples and 7722 psi for the tensile samples. Note, however, that due to the orientation of the braze joint relative to the load direction. the shear samples were capable of carrying more than twice the load of the tensile samples. Inspection of the bonding surfaces of the destroyed samples indicated 
that each bond joint was only $20 \%$ to $30 \%$ wetted. Discussions with the manufacturer revealed that the samples were not manufactured with the Class A brazing preparation. The prototype pylon, flight articles and their respective validation samples will be manufactured to this specification and certification of this process will be provided.

\section{Conclusions}

While the qualification braze joints were not manufactured to the level of quality originally anticipated, their strength was sufficient to provide the USA gimbal structure design with a positive margin of safety. One prototype pylon, two flight pylons and one flight yoke will now be built by the Aluminum Dip Braze Co.

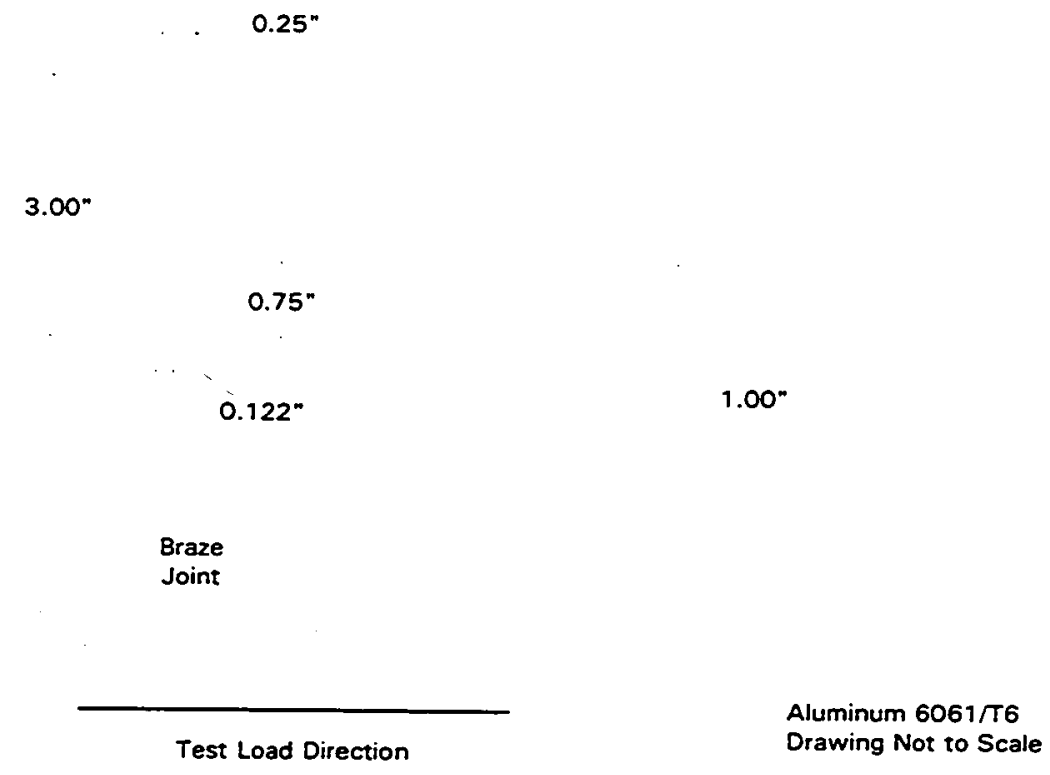

Figure 1: Shear Sample Specifications 
$2.00^{*}$

$1.00^{*}$

Braze

Joint

Test Load Direction

Aluminum 6061/T6

Drawing Not to Scale

Figure 2: Tensile Sample Specifications

$$
1.75^{\prime \prime}
$$

$0.50^{\prime \prime}$

\section{Braze Area}

Test Load Direction

Aluminum $6061 / \pi 6$

Drawing Not to Scale

Figure 3: Final Shear Sample Configuration 


\begin{tabular}{|c|c|c|r|r|r|r|}
\hline Sample & $\begin{array}{c}\text { Width } \\
\text { (in) }\end{array}$ & $\begin{array}{c}\text { Thickness } \\
\text { (in) }\end{array}$ & $\begin{array}{c}\text { Area } \\
\text { (sq. in.) }\end{array}$ & $\begin{array}{c}\text { Failure } \\
\text { Load } \\
\text { (lb) }\end{array}$ & $\begin{array}{c}\text { Failure } \\
\text { Stress } \\
\text { (psi) }\end{array}$ & $\begin{array}{c}\text { Shear } \\
\text { Stress } \\
\text { (psi) }\end{array}$ \\
\hline 10 & 0.503 & 0.241 & 0.121 & 2200 & 18148 & 5832 \\
\hline 12 & 0.500 & 0.242 & 0.121 & 2950 & 24380 & 7867 \\
\hline 13 & 0.501 & 0.239 & 0.120 & 2760 & 23050 & 7345 \\
\hline 14 & 0.504 & 0.238 & 0.120 & 2150 & 17924 & 5688 \\
\hline 15 & 0.499 & 0.240 & 0.120 & 2150 & 17953 & 5745 \\
\hline 17 & 0.500 & 0.240 & 0.120 & 2900 & 24167 & 7733 \\
\hline 18 & 0.503 & 0.240 & 0.121 & 2900 & 24023 & 7687 \\
\hline 19 & 0.501 & 0.239 & 0.120 & 2720 & 22716 & 7239 \\
\hline & & & & 2961 & 21545 & 6892 \\
\hline
\end{tabular}

Table la: Shear Sample Results

\begin{tabular}{|c|c|c|r|r|r|}
\hline Sample & $\begin{array}{c}\text { Width } \\
\text { (in) }\end{array}$ & $\begin{array}{c}\text { Thickness } \\
\text { (in) }\end{array}$ & $\begin{array}{c}\text { Area } \\
\text { (sq. in.) }\end{array}$ & $\begin{array}{c}\text { Failure } \\
\text { Load } \\
\text { (lb) }\end{array}$ & $\begin{array}{c}\text { Failure } \\
\text { Stress } \\
\text { (psi) }\end{array}$ \\
\hline 10 & 0.501 & 0.235 & 0.118 & 950 & 8069 \\
\hline 11 & 0.499 & 0.247 & 0.123 & 1160 & 9412 \\
\hline 12 & 0.504 & 0.247 & 0.124 & 690 & 5543 \\
\hline 14 & 0.503 & 0.245 & 0.123 & 1210 & 9819 \\
\hline 15 & 0.502 & 0.243 & 0.122 & 1020 & 8362 \\
\hline 16 & 0.503 & 0.246 & 0.124 & 890 & 7193 \\
\hline 17 & 0.501 & 0.248 & 0.123 & 770 & 6197 \\
\hline 19 & 0.500 & 0.245 & 0.123 & 880 & 7184 \\
\hline & & & Average & 946 & 7722 \\
\cline { 4 - 7 } & & & Std Dev & 179 & 1484 \\
\hline
\end{tabular}

Table Ib: Tensile Sample Results

Notes:

1. Samples constructed of $\mathrm{Al} 6061 / \mathrm{T} 6$

2. Al $6061 / \mathrm{T} 6$ has an ultimate strength of $45,000 \mathrm{psi}$

3. All failure were brittle fracture of the joint

4. Error in failure loads due to testing $-10 \%$ of nominal 


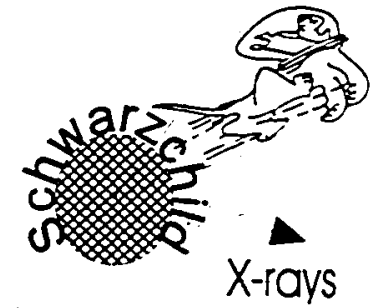

Astrogravity Note \# 19 John Hanson

SLAC

March 14, 1996

\section{USA Structure Quality Assurance Testing}

\section{Introduction}

The primary support structure for the Unconventional Stellar Aspect Experiment makes extensive use of a bonding process known as dip-brazing in its manufacturing. Two pylons and one yoke are to be assembled from components made of 6061/T6 aluminum that are dip-brazed together. In order to ensure that dip-brazed joints will have sufficient strength to survive the launch environment. a number of qualification braze joints were tested.

\section{Test Samples}

Qualification pull samples were manufactured with each prototype and flight article. Five shear samples were tested with each item (see Figure 1). All samples were made from $6061 / \mathrm{T} 6$ aluminum. The test samples were dip-brazed by the Aluminum Dip Braze Co. of Burbank. Ca. All manufacturing was done to Mil-B-7883-B Type V Grade $B$ with joint preparation to Mil-B-7883-B Type V Grade A. A total of four sets of five samples were made.

\section{Test Results}

All samples were tested to failure using an Instron Model 1125 tensile test machine in the Material Science Department at Stanford University. This machine had a maximum applied load of $20,000 \mathrm{lb}$ and was calibrated at $10,000 \mathrm{lb}$. All test sample failure modes were brittle fracture of the braze joint. the resulting failure loads are summarized in Table 1 . The mean failure loads will be used in the final structural analysis report to determine the design margin of safety. 
3.00"

$0.75^{*}$

$0.122^{\prime \prime}$

$1.00^{\circ}$

Braze

Joint

Test Load Direction

Aluminum $6061 \pi 6$

Drawing Not to Scale

Figure 1: Shear Sample Specifications 


\begin{tabular}{|c|c|c|r|r|r|r|r|r|}
\hline Sample & $\begin{array}{c}\text { Depth } \\
\text { (in) }\end{array}$ & $\begin{array}{c}\text { Width } \\
\text { (in) }\end{array}$ & $\begin{array}{c}\text { Thickness } \\
\text { (in) }\end{array}$ & $\begin{array}{c}\text { X-Section } \\
\text { Area } \\
\text { (in^2) }\end{array}$ & $\begin{array}{c}\text { Braze } \\
\text { Area } \\
\text { (in^2) }\end{array}$ & $\begin{array}{c}\text { Failure } \\
\text { Load } \\
\text { (lb) }\end{array}$ & $\begin{array}{c}\text { Shear } \\
\text { Stress } \\
\text { (psi) }\end{array}$ & $\begin{array}{c}\text { Tensile } \\
\text { Stress } \\
\text { (psi) }\end{array}$ \\
\hline 1 & 0.75 & 1.00 & 0.25 & 0.25 & 0.75 & 4500 & 6000 & 18000 \\
\hline 2 & 0.75 & 1.00 & 0.25 & 0.25 & 0.75 & 4820 & 6427 & 19280 \\
\hline 3 & 0.75 & 1.00 & 0.25 & 0.25 & 0.75 & 6350 & 8467 & 25400 \\
\hline 4 & 0.75 & 1.00 & 0.25 & 0.25 & 0.75 & 4460 & 5947 & 17840 \\
\hline 5 & 0.75 & 1.00 & 0.25 & 0.25 & 0.75 & 6020 & 8027 & 24080 \\
\hline & \multicolumn{10}{|c|}{ Average } & 5230 & 6973 & 20920 \\
\hline
\end{tabular}

Table 1a: Pylon \#1 Qualification Samples

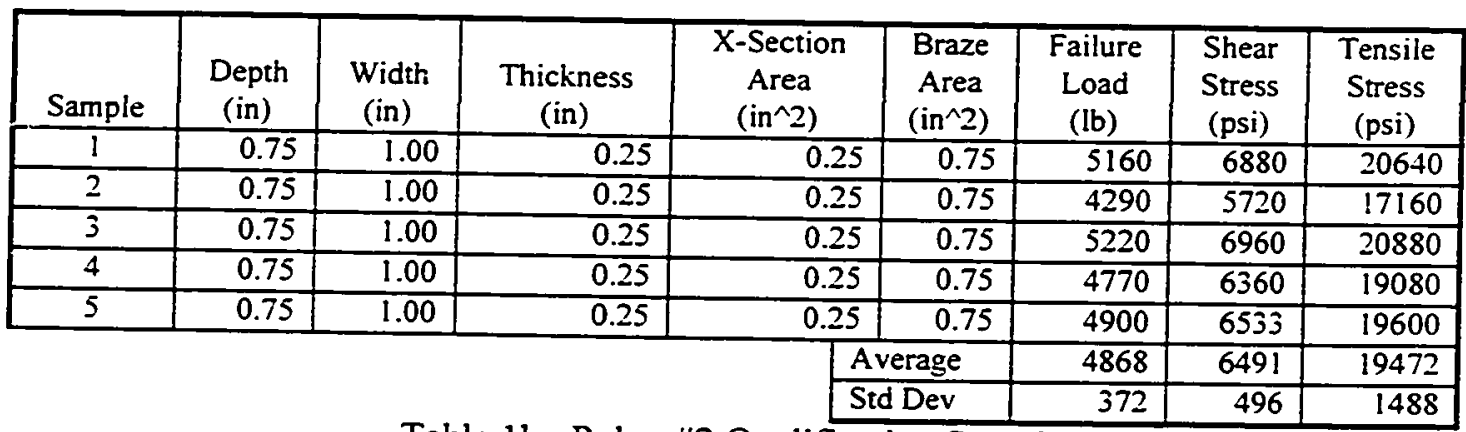

Table 1b: Pylon \#2 Qualification Samples

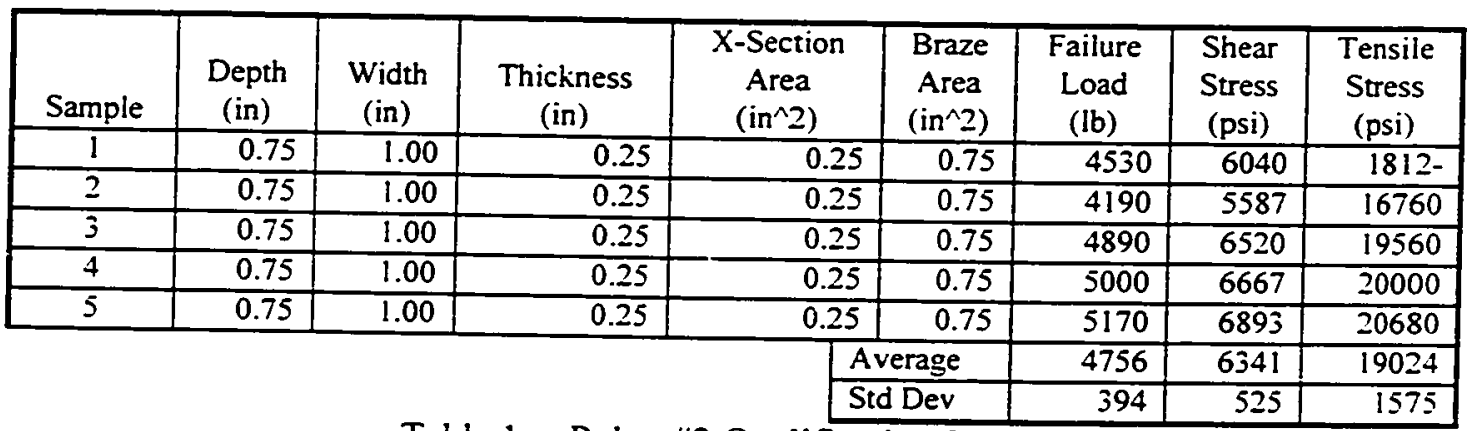

Table 1c: Pylon $\# 3$ Qualification Samples

\begin{tabular}{|c|c|c|r|r|r|r|r|r|}
\hline Sample & $\begin{array}{c}\text { Depth } \\
\text { (in) }\end{array}$ & $\begin{array}{c}\text { Width } \\
\text { (in) }\end{array}$ & $\begin{array}{c}\text { Thickness } \\
\text { (in) }\end{array}$ & $\begin{array}{c}\text { X-Section } \\
\text { Area } \\
\text { (in^2) }\end{array}$ & $\begin{array}{c}\text { Braze } \\
\text { Area } \\
\text { (in^2) }\end{array}$ & $\begin{array}{c}\text { Failure } \\
\text { Load } \\
\text { (lb) }\end{array}$ & $\begin{array}{c}\text { Shear } \\
\text { Stress } \\
\text { (psi) }\end{array}$ & $\begin{array}{c}\text { Tensile } \\
\text { Stress } \\
\text { (psi) }\end{array}$ \\
\hline 1 & 0.75 & 1.00 & 0.25 & 0.25 & 0.75 & 5920 & 7893 & 23680 \\
\hline 2 & 0.75 & 1.00 & 0.25 & 0.25 & 0.75 & 4190 & 5587 & 16760 \\
\hline 3 & 0.75 & 1.00 & 0.25 & 0.25 & 0.75 & 3462 & 4613 & 13840 \\
\hline 4 & 0.75 & 1.00 & 0.25 & 0.25 & 0.75 & 4760 & 6347 & 19040 \\
\hline 5 & 0.75 & 1.00 & 0.25 & 0.25 & 0.75 & 4310 & 5747 & 17240 \\
\hline
\end{tabular}

Table 1d: Yoke Qualification Samples 


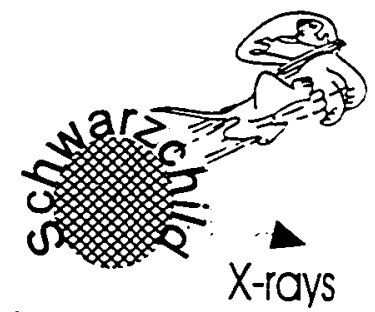

Astrogravity Note \# 20 John Hanson

SLAC

March 14, 1996

\section{USA Pylon Static Load Test}

\section{Introduction}

The Unconventional Stellar Aspect Experiment (USA) is an X-ray detector that will be launched on an Air Force sponsored satellite called the Advanced Research and Global Observation Satellite (ARGOS). USA is a joint experiment between the Naval Research Laboratory (NRL) and the Stanford Linear Accelerator Center (SLAC).

Under the direction of Professor Elliott Bloom. SLAC has undertaken the design. fabrication and testing of the USA gimbal structure (Figure 1). This structure consists of two outer pylons that allow a yoke to pivot in the pitch axis between them. The proportional chambers are gimballed inside the yoke to allow motion in the yaw axis. In order to make an efficient design. extensive finite element modelling was used in the design process. Additionally, a process relatively new to the aerospace industry. called dipbrazing, was used to make the structure as light as possible. A prototype of the hot-side pylon was fabricated first and a static load test was performed. This test approximated the flight loads expected to be seen by the USA structure.

\section{Static Load test Concept} below.

The maximum static loads seen by the USA experiment are defined in USA ICD Table 1, repeated

\begin{tabular}{|l|c|c|}
\hline & Axial (g's) & Lateral (g's) \\
\hline Lift Off & $+3.25 /-0.26$ & $+/-4.00$ \\
\hline Main Engine Cutoff & +7.1 & $+/-0.13$ \\
\hline
\end{tabular}

Table 1: USA Acceleration Environment

The USA ICD also specifies that the design for any structural element that will not be subjected to a static load test use a factor of safety of 2.0 and provide positive margin in the worst case load. Any element tested to be $115 \%$ of the ICD loads need only be designed witha factor of safety of 1.5 and positive margin. All structural elements of USA were designed with a factor of safety of 2.0 and positive margin. The prototypepylon was the tested to $115 \%$ of the ICD loads. The resulting worst case stress conditions for the hot-side pylon are detailed in Table 2 and shown pictorially in Figures 2 through 5 . Noting that the lift off condition has the highest peak stress. the static load test was performed for this load case only.

\begin{tabular}{|c|c|c|c|c|}
\hline & \multicolumn{3}{|c|}{ Acceleration (in $/ \mathrm{s}^{\wedge} 2$ ) } & \multirow{2}{*}{$\begin{array}{c}\text { Max Stress } \\
\text { Von Mises (psi) }\end{array}$} \\
\hline Load Case & $a_{x}$ & $\mathrm{a} y$ & $\overline{a_{z}}$ & \\
\hline
\end{tabular}




\begin{tabular}{|l|c|c|c|c|}
\hline Lift Off & -1444 & -1750 & 309 & 1160 \\
\hline MECO & -3155 & 41 & -41 & 485 \\
\hline
\end{tabular}

Table 2: Worst Case Stress Conditions

These acceleration loads composed of two components:

1. A point load and moment at the motor/yoke interface due to the support load for the yoke/detector assembly and the acceleration load on the motor assembly.

2. A distributed load on the entire pylon due to self-loading in the acceleration environment.

Self-loading of the pylon was neglected during the static load test. the resulting point loads are given in Table 3. These loads act at the center of the pylon/motor interface ring.

\begin{tabular}{|l|l|c|c|c|c|c|c|}
\cline { 2 - 8 } \multicolumn{1}{c|}{} & \multicolumn{3}{c|}{ Force (lb) } & \multicolumn{3}{c|}{ Moment (in Ib) } \\
\cline { 2 - 8 } \multicolumn{1}{c|}{} & Node & $\mathrm{F}_{\mathrm{X}}$ & $\mathrm{F}_{\mathrm{y}}$ & $\mathrm{F}_{\mathrm{z}}$ & $\mathrm{M}_{\mathrm{X}}$ & $\mathrm{M}_{\mathrm{y}}$ & $\mathrm{M}_{\mathrm{z}}$ \\
\hline Hub Load & 13955 & 328 & 344 & -183 & -121 & 0 & 1830 \\
\hline Motor Load & 00060 & 71 & 86 & -15 & 0 & 0 & 0 \\
\hline Total Load & 12857 & 399 & 430 & -198 & -274 & 0 & 1579 \\
\hline
\end{tabular}

Table 3: Worst Case (-Y) Pylon Point Loads

\section{Test Apparatus}

The point load was applied to the test article by attaching two cables to the pylon and placing these cables in tension. Any desired force and moment combination can be generated by applying two different forces at two different locations. These loads and their points of application are given in Table 4. These are given relative to the center of the pylon/motor interface ring.

\begin{tabular}{|c|c|c|c|c|c|c|}
\hline & \multicolumn{3}{|c|}{ Force (lb) } & \multicolumn{3}{|c|}{ Location (in) } \\
\hline & $F_{x}$ & $F_{y}$ & $\overline{F_{z}}$ & $r_{x}$ & $r_{y}$ & $r_{z}$ \\
\hline Load 1 & 223 & 0 & -198 & -1.53 & -7.08 & -1.72 \\
\hline Load 2 & 176 & 430 & 0 & 1.25 & 3.06 & 3.90 \\
\hline Total Load & 399 & 430 & -198 & & & \\
\hline
\end{tabular}

Table 4: Test Case Maximum Applied Loads (at Node 12857) 


\section{Test Apparatus}

The test apparatus consists of six components:

I. Test aricle: The prototype pylon

2. Aluminum mounting plate: $0.5^{\prime \prime}$ thick mounting plate to interface between the pylon and the workbench top.

3. Cable/motor interface assembly: Machined at SLAC and providing an interface for two separate loads to be applied simultaneously to the assembly.

4. Load cell: Two load cells borrowed from NASA/Ames Research Center. Each cell outputs a voltage proportional to the load acting along the sensitive axis of the cell. the voltage was recorded with a digital multimeter (DMM).

5. Tumbuckle assembly: Two assemblies purched from West Marine with working loads of $2000 \mathrm{lb}$.

6. Dial indicator: Five dial indicators will be arranged to measure the deflections of the motor/yoke interface.

The pylon was bolted to the aluminum plate which was bolted to a workbench top. The static load was applied by attaching the cable/motor interface assembly to the pylon with the cable assemblizs arranged to apply two forces at two non-coincident points on the motor interface. A turnbuckle was placed on each cable to apply a desired load in the direction of the cable. A load cell on each cable measured the actual force applied. This is described pictorially in Figure 6.

Six dial indicators were used to measure the deflection of the pylon head relative to the aluminum mounting plate. These were arranged so that the three rotations and three deflections that describe the motion of the pylon head could be determined. Additionally, one dial indicator ( $\# 7)$ was used to measure the deflection of the baseplate relative to the workbench. The locations of the dial indicators are summarized in Table 5 and shown pictorially in Figure 7.

\begin{tabular}{|c|c|c|c|c|}
\hline \multirow{2}{*}{ Measurement $\#$} & \multirow{2}{*}{$\begin{array}{c}\text { Measurement } \\
\text { Direction }\end{array}$} & $\mathrm{X}$ & $\mathrm{Y}$ & $\mathrm{Z}$ \\
\cline { 3 - 5 } & $\mathrm{x}$ & -3.65 & 0.05 & 4.57 \\
\hline $\mathrm{I}$ & $\mathrm{x}$ & -3.65 & 0.01 & -4.38 \\
\hline 2 & $-z$ & -1.98 & -0.80 & -4.93 \\
\hline 3 & $\mathrm{y}$ & -3.11 & 0.31 & 0.69 \\
\hline 4 & $\mathrm{y}$ & 3.04 & 0.31 & -1.97 \\
\hline 5 & $\mathrm{y}$ & 2.60 & 0.31 & 1.96 \\
\hline 6 & $y$ & 21.13 & 17.75 & -12.00 \\
\hline 7 & & & \\
\hline
\end{tabular}

Table 5: Measurement Locations

\section{Test Results}

The static load test of the prototype pylon was performed in five separate cycles. Each cyle involved increasing the test load from $0 \%$ to $100 \%$ of the peak load (as given in Table 4 ) in increments of $10 \%$. After reaching 100\%. the test loads were decreased in decrements of $10 \%$ back to zero load. Each load was achieved as follows: 
1. Tighten turmbuckles while reading the voltage output of each load cell through the DMM

2. Read and record the measurments of each dial indicator as well as the voltage of each load cell.

The test loads and their corresponding load cell output voltages are summarized in Table 6 . The measured deflections are attached. Note that five load cycles, each increments to $100 \%$ of the test load and the decremented to zeo load. did not result in any brittle fracture of the dip brazed joints or plastic deformation of the aluminum plates.

\section{Analysis of Deflections}

A finite element of the prototype pylon in the test configuration was analyzed with the IDEAS software package. The resulting stress distribution is shown in Figure 8 while the expected deflection is shown in Figure 9. Table 7 describes the expected deflections of the pylon head.

\begin{tabular}{|c|c|}
\hline Component & $\begin{array}{c}\text { Predicted } \\
\text { Deflection (in) }\end{array}$ \\
\hline$\Delta \mathrm{X}$ & 0.014 \\
\hline$\Delta \mathrm{Y}$ & 0.049 \\
\hline$\Delta \mathrm{Z}$ & -0.002 \\
\hline
\end{tabular}

Table 7: Predicted Pylon Head Deflections

\begin{tabular}{|c|c|}
\hline & $\begin{array}{c}\text { Predicted } \\
\text { Deflection (mrad) }\end{array}$ \\
\hline$\Delta \Theta_{x}$ & -0.0337 \\
\hline$\Delta \Theta_{y}$ & -0.0926 \\
\hline$\Delta \Theta_{z}$ & -2.218 \\
\hline
\end{tabular}

It is assumed that the pylon head undergoes small angle rigid body rotation and rigid body deflection. the deflections predicte by the finite element model can be related easily to the six measurements. The deflection of a point $P^{\prime}$ in such a body can be releted to the deflection of another point $P$ in that body and the rotation of the body about $P$ as follows:

$$
\left\{\begin{array}{l}
\Delta X \\
\Delta Y \\
\Delta Z
\end{array}\right\}_{P^{\prime}}=\left\{\begin{array}{l}
\Delta X \\
\Delta Y \\
\Delta Z
\end{array}\right\}_{P}+\left[\begin{array}{ccc}
0 & z & -y \\
-z & 0 & x \\
y & -x & 0
\end{array}\right]\left\{\begin{array}{l}
\Delta \Theta_{x} \\
\Delta \Theta_{y} \\
\Delta \Theta_{z}
\end{array}\right\}
$$

where

$(x, y, z)=$ location of $P^{\prime}$ relative to $P$

$\left(\Delta \Theta_{\gamma}, \Delta \Theta_{y} . \Delta \theta_{z}\right)=$ rotation about $P$

$(\Delta \mathrm{X}, \Delta \mathrm{Y}, \Delta \mathrm{Z})_{\mathrm{P}} \cdot=$ deflection of $\mathrm{P}^{\prime}$

$(\Delta X, \Delta Y, \Delta Z)_{P}=$ deflection of $P$

The six deflections (each measured at a different point on the pylon head and in a different direction) are related to the three deflections and three rotations of the pylon head measured relative to the center of the pylon/motor interface ring as follows:

$$
\left\{\begin{array}{c}
\Delta M_{1} \\
\Delta M_{2} \\
-\Delta M_{3} \\
\Delta M_{4} \\
\Delta M_{5} \\
\Delta M_{6}
\end{array}\right\}=\left[\begin{array}{lll}
1 & 0 & 0 \\
1 & 0 & 0 \\
0 & 0 & 1 \\
0 & 1 & 0 \\
0 & 1 & 0 \\
0 & 1 & 0
\end{array}\right]\left\{\begin{array}{l}
\Delta X \\
\Delta Y \\
\Delta Z
\end{array}\right\}_{P}+\left[\begin{array}{ccc}
0 & 4.57 & -0.05 \\
0 & -4.38 & -0.01 \\
-0.80 & 1.98 & 0 \\
-0.69 & 0 & -3.11 \\
1.97 & 0 & 3.04 \\
-1.96 & 0 & 2.60
\end{array}\right]\left\{\begin{array}{l}
\Delta \Theta_{x} \\
\Delta \Theta_{y} \\
\Delta \Theta_{z}
\end{array}\right\}_{P}
$$


The resulting predicted deflections at the measurement locations are given in Table 8 along with the measured peak deflections, averaged over the five load cycle tests. The final or "zero load" deflections are also shown.

\begin{tabular}{|c|c|c|c|c|c|}
\hline & \multicolumn{2}{|c|}{$\begin{array}{c}\text { Peak Deflection } \\
\left(0.001^{\prime \prime}\right)\end{array}$} & \multicolumn{2}{c|}{$\begin{array}{c}\text { Final Deflection } \\
\left(0.0011^{\prime \prime}\right.\end{array}$} & $\begin{array}{c}\text { Predicted } \\
\text { Deflection } \\
\left(0.001^{*}\right)\end{array}$ \\
\hline$\#$ & Mean & Std Dev & Mean & Std Dev & Mean \\
\hline 1 & 14 & 1.0 & 1.0 & 1.3 & 15 \\
\hline 2 & 6 & 0.6 & 0.4 & 0.6 & 14 \\
\hline 3 & 2 & 0.2 & 0.1 & 0.2 & 2 \\
\hline 4 & 68 & 3.7 & 3.0 & 4.1 & 56 \\
\hline 5 & 36 & 2.1 & 1.8 & 2.4 & 42 \\
\hline 6 & 42 & 2.3 & 1.7 & 2.5 & 43 \\
\hline
\end{tabular}

The elastic nature ofthe deflections is shown in Figures 10 through 15.

Conclusions

The prototype pylon for the USA experiment was tested to $115 \%$ of the USA ICD flight loads in a static load test. The dip brazed joints did not undergo any brittle failure. the pylon did not undergo any plastic deformation. the measure deflections matched to expected deflections to within ten percent. 


\section{Appendix C: Pointing Feasibility Study - C Procedures}

\section{C.1 Sensitivity Study}

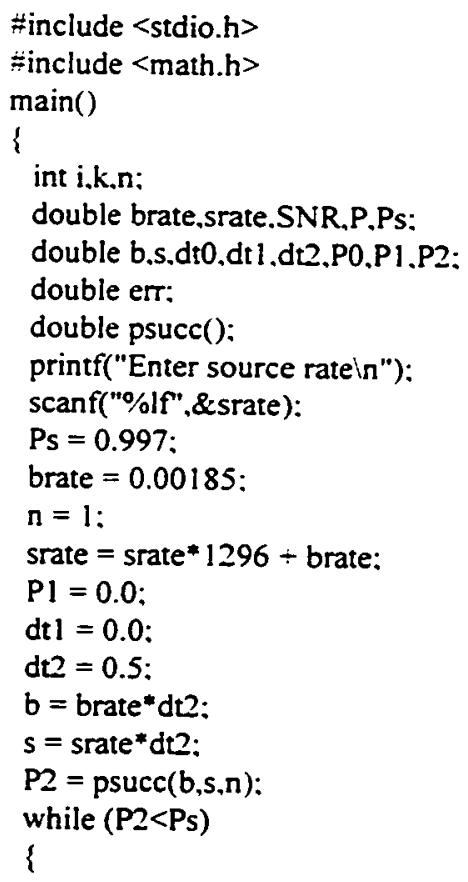




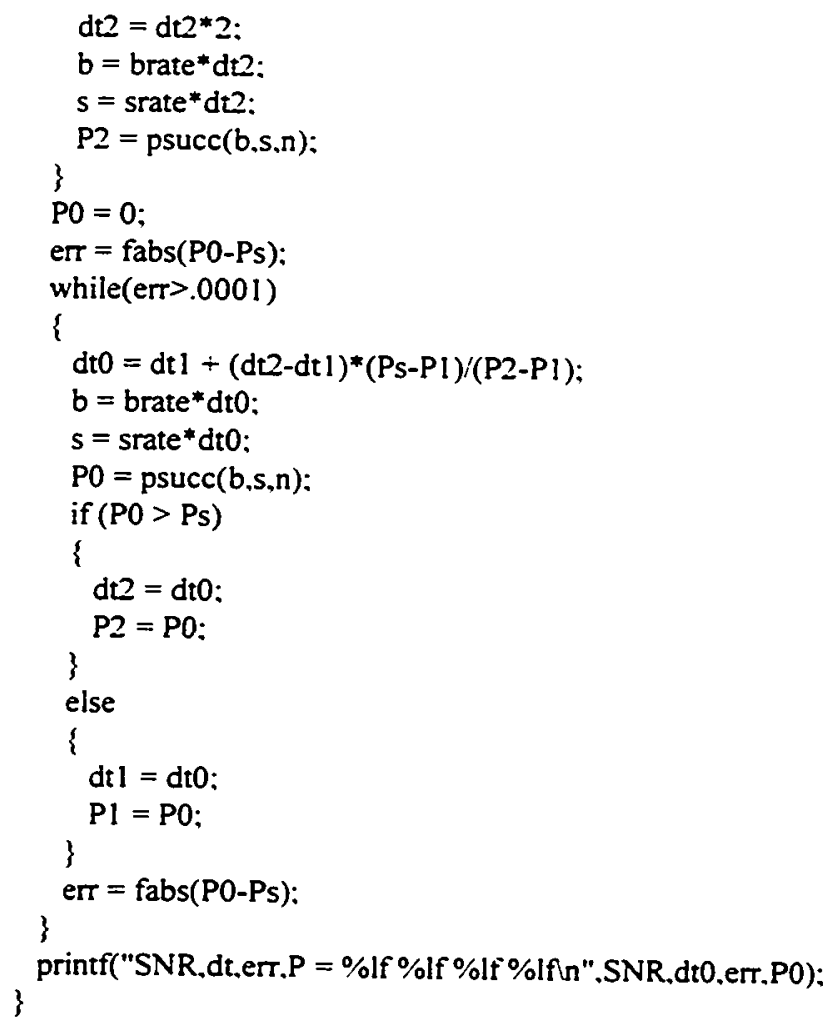




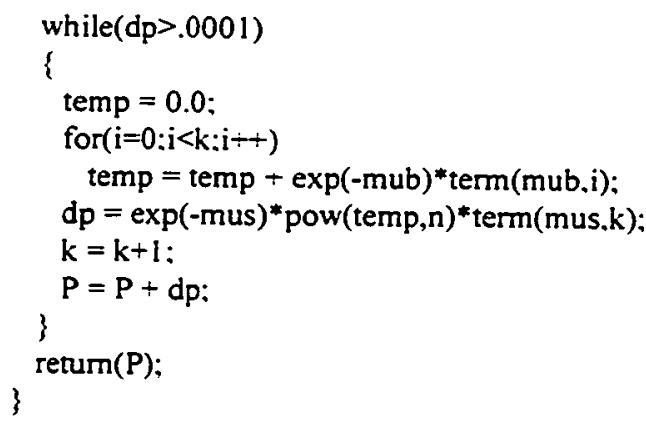

\section{C.2 X-ray Sky Survey}

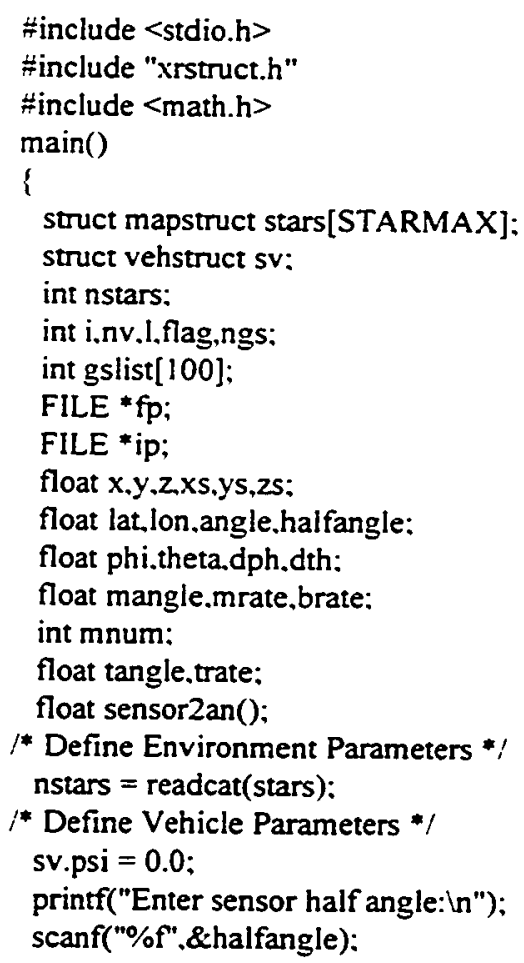




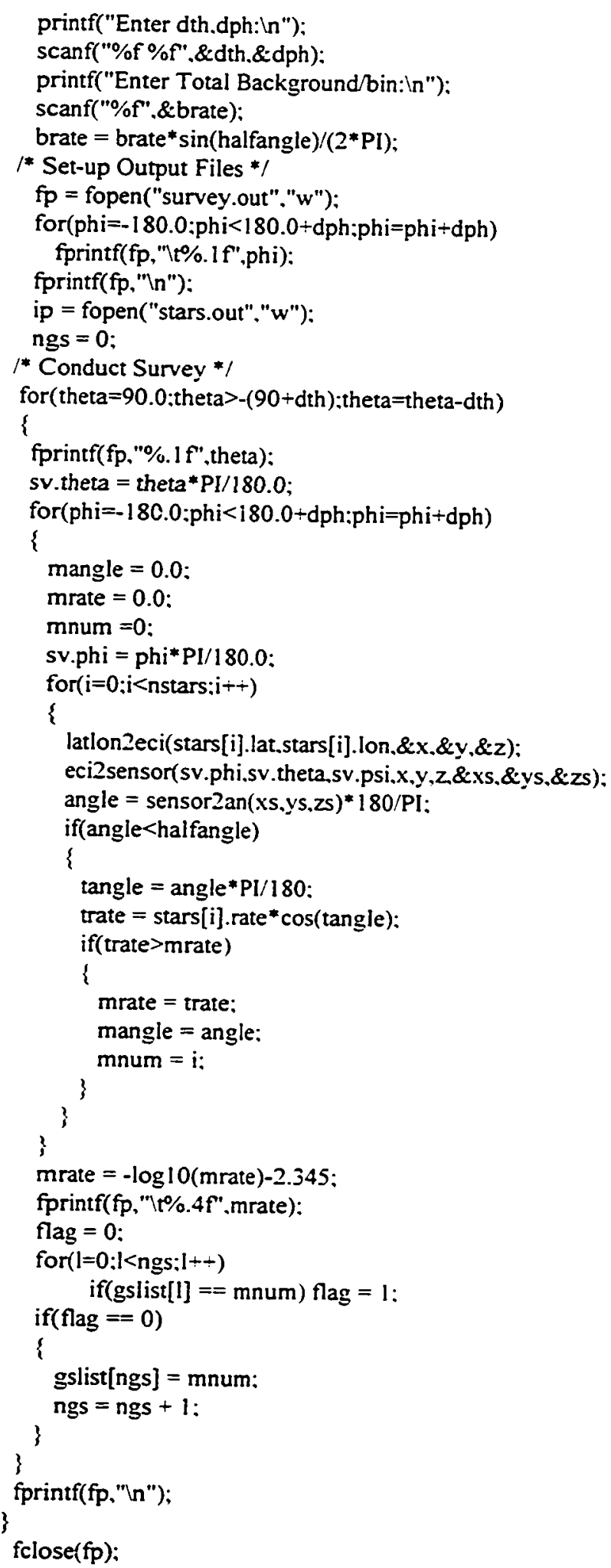




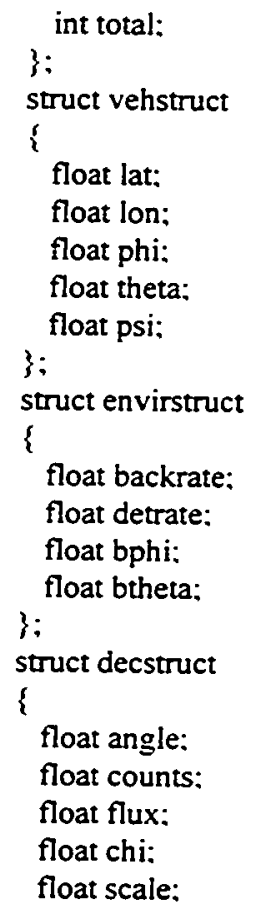




\section{Appendix D: Derivation of Equations Describing Collimator Transmission}

\section{D.1 Two Dimensional Collimators}

The response of a one dimensional collimator can be described in terms of a single angle, $\alpha$, as:

$$
T_{c}=\left(1-\frac{\tan |\alpha|}{\tan \left(\alpha_{0}\right)}\right) \cos (\alpha)
$$

Physically, the term in parentheses represents the shadowing of the detector surface by the sides of the collimator. The $\cos (\alpha)$ term represents the effect of projecting the detector area into the direction of the x-ray source. further reducing the effective detector area.

A two dimensional collimator can be manufactured by placing a rectangular shroud around the detector. The response of this collimator can then be described in 
terms of two angles, $\alpha$ and $\rho$, that are the angles made by projecting the direction to the $\mathrm{x}$-ray source onto the $\mathrm{xz}$ and $\mathrm{yz}$ planes respectively (Figure D.1), viz.:

$$
T_{c}=\left(1-\frac{\tan |\alpha|}{\tan \left(\alpha_{0}\right)}\right)\left(1-\frac{\tan |\rho|}{\tan \left(\rho_{0}\right)}\right) \cos (\gamma)
$$

Note that the reduction in collimator response due to shadowing is described by functions of $\alpha$ and $\rho$. The $\cos (\gamma)$ term represents the projection of the detector area on the $x$-ray direction and is a function of $\alpha$ and $\rho$, viz.:

$$
\cos (\gamma)=\frac{1}{\sqrt{1+\tan ^{2}(\alpha)+\tan ^{2}(\rho)}}
$$

We would like to separate the collimator transmission into the product of the transmission of two one dimensional collimators acting independently and oriented orthogonally. To this end we can write the $\cos (\mathrm{g})$ term as.

$$
\cos (\gamma)=\cos (\alpha) \cos (\rho) \frac{1}{\sqrt{\sin ^{2}(\rho) \cos ^{2}(\alpha)+\cos ^{2}(\rho)}}
$$

The transmission of a two dimensional rectangular collimator can then be written as (using the fact that $\cos ^{2} \alpha+\sin ^{2} \alpha=1$ ):

$$
T_{c}(\alpha, \rho)=T_{c}(\alpha) T_{c}(\rho) \frac{1}{\sqrt{1-\sin ^{2}(\rho) \sin ^{2}(\alpha)}} \cong T_{c}(\alpha) T_{c}(\rho)
$$

For collimators with reasonably small fields of view, the $\frac{1}{\sqrt{1-\sin ^{2}(\rho) \sin ^{2}(\alpha)}}$ term is going to be very nearly one. As an example. consider the HEAO-AI Module 3 collimator in which the largest value of $\alpha$ is four degrees and the largest value of $\rho$ is one degree. In this case, the error in the computed transmission caused by assuming this term is unity is $7.4\left(10^{-7}\right)$. 


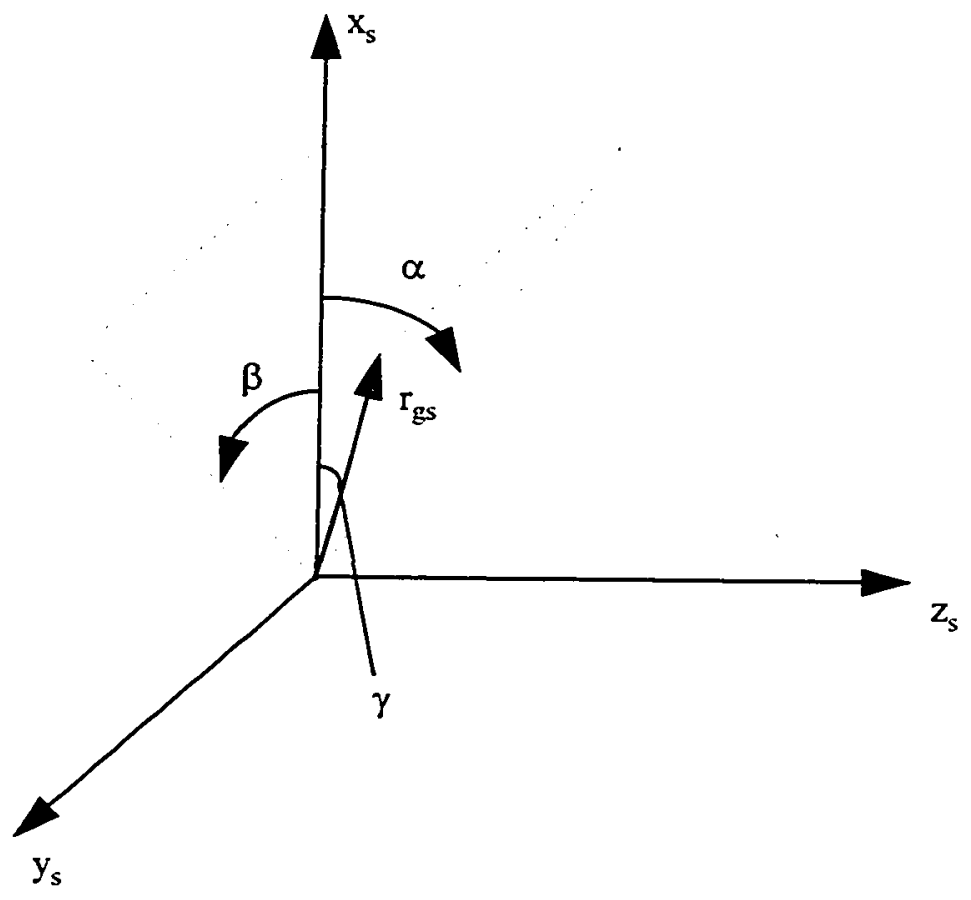

Figure D.1: Collimator Angle Definitions 


\section{Appendix E: First Order Star Scanner Model}

\section{E.1 Coordinate Systems}

Celestial Coordinates - Inertially fixed frame. Denoted $\left(X_{c}, Y_{c}, Z_{c}\right)$, the $X_{c}$ axis points to galactic north while the $Y_{c}$ axis is in the direction of the first point of Aries.

Guide Star Coordinates - Inertially fixed frame. A guide star is defined by its position in celestial coordinates. This position is given by the right ascension of the ascending node (RA) and the declination (DEC) of the star (Figure E.1). First. a rotation through RA about the $\mathrm{X}_{\mathrm{c}}$ axis to a set of coordinates $\left(\mathrm{X}^{*}, \mathrm{Y}^{*}, \mathrm{Z}^{*}\right)$ is performed. A rotation through DEC about the $Z^{\prime}$ axis to the guide star coordinates $(X, Y, Z)$ is then made. This places the $\mathrm{Y}$ axis pointing directly at the guide star. Derivation of all equations of motion will take place in the Guide Star inertial frame. 
Spacecraft Coordinates - Body fixed frame. The spacecraft coordinates are in the principal directions of the inertia tensor. The $z$-axis is in direction of the principal axis with the maximum inertia and is in the nominal spin direction. This system is defined by the unit vectors $(x, y, z)$.

Instrument Coordinates - Body fixed frame. The $y_{i}$-axis is in the direction of maximum instrument transmission. The $x_{i}$-axis defines the pitch axis of the instrument. The $z_{i}$-axis defines the roll axis of the instrument.

\section{E.2 Spacecraft Orientation}

The orientation of the spacecraft relative to guide star coordinates can be described by three Euler angles that represent the rotation of the spacecraft body fixed coordinate system in guide star coordinates. In this discussion these will be the yaw, pitch and roll angles $(\psi, \theta, \phi)$ (Figure E.2).

Yaw is a rotation about the $Y$-axis to a coordinate system $\left(x^{*}, y^{*}, z^{\prime}\right)$ :

$$
\begin{aligned}
& x^{\prime}=X \cos \psi-Z \sin \psi \\
& y^{\prime}=Y \\
& z^{\prime}=X \sin \psi+Z \cos \psi
\end{aligned}
$$

Pitch is a rotation about the $x^{\prime \prime}$-axis to a coordinate system $\left(x^{\prime \prime}, y^{\prime \prime} . z^{\prime \prime}\right)$ :

$$
\begin{aligned}
& x^{\prime \prime}=x^{\prime} \\
& y^{\prime \prime}=y^{\prime} \cos \theta+z^{\prime} \sin \theta \\
& z^{\prime \prime}=-y^{\prime} \sin \theta+z^{\prime} \cos \theta
\end{aligned}
$$

Roll is a rotation about the $z "$-axis to the spacecraft coordinate system $(x, y, z)$ :

$$
\begin{aligned}
& x=x^{\prime \prime} \cos \phi+y^{\prime \prime} \sin \phi \\
& y=-x^{\prime \prime} \sin \phi+y^{\prime \prime} \cos \phi \\
& z=z^{\prime \prime}
\end{aligned}
$$


The transformation from spacecraft coordinates to guide star coordinates can be written as:

$$
A_{s c 2 g s}=\left[\begin{array}{ccc}
\cos \psi \cos \phi+\sin \psi \sin \theta \sin \phi & \cos \theta \sin \phi & -\sin \psi \cos \phi+\cos \psi \sin \theta \sin \phi \\
-\cos \psi \sin \phi+\sin \psi \sin \theta \cos \phi & \cos \theta \cos \phi & \sin \psi \sin \phi+\cos \psi \sin \theta \cos \phi \\
\sin \psi \cos \theta & -\sin \theta & \cos \psi \cos \theta
\end{array}\right]
$$

Conversely, the transformation from guice star coordinates to spacecraft coordinates can be written as:

$$
A_{g s 2 s c}=\left[\begin{array}{ccc}
\cos \psi \cos \phi+\sin \psi \sin \theta \sin \phi & -\cos \psi \sin \phi+\sin \psi \sin \theta \cos \phi & \sin \psi \cos \theta \\
\cos \theta \sin \phi & \cos \theta \cos \phi & -\sin \theta \\
-\sin \psi \cos \phi+\cos \psi \sin \theta \sin \phi & \sin \psi \sin \phi+\cos \psi \sin \theta \cos \phi & \cos \psi \cos \theta
\end{array}\right]
$$

\section{E.3 Instrument Orientation}

In some cases the instrument axes may not be perfectly oriented with the spacecraft axes. This may be due to the purposeful placement of the instrument or due to misalignments during manufacturing. The process of defining the instrument axes relative to the spacecraft axes in terms of euler angles is similar to that discussed in Section E.2. In this case. the euler angles are denoted $\left(\delta_{x}, \delta_{y}, \delta_{z}\right)$. In this case the rotations go $\left(\delta_{z}, \delta_{x}, \delta_{y}\right)$ (Figure E.3).

$\delta_{z}$ is a rotation about the $z$-axis to a coordinate system $\left(x_{i}{ }^{\circ}, y_{i}, z_{i}\right)$ :

$$
\begin{aligned}
& x_{i}^{\prime}=x \cos \delta_{x}+y \sin \delta_{x} \\
& y_{i}^{\prime}=-x \sin \delta_{x}+y \cos \delta_{x} \\
& z_{i}^{\prime}=z
\end{aligned}
$$


$\delta_{\mathbf{x}}$ is a rotation about the $x_{i}{ }^{\prime}$-axis to a coordinate system $\left(x_{i}{ }^{*}, y_{i}{ }^{\prime}, z_{i}{ }^{\prime \prime}\right)$ :

$$
\begin{aligned}
& x_{i}^{\prime \prime}=x^{\prime} \\
& y_{i}^{\prime \prime}=y_{i}^{\prime} \cos \delta_{x}+z_{i}^{\prime} \sin \delta_{x} \\
& z_{i}^{\prime \prime}=-y_{i}^{\prime} \sin \delta_{x}+z_{i}^{\prime} \cos \delta_{x}
\end{aligned}
$$

$\delta_{y}$ is a rotation about the $y_{i}$ "-axis to the spacecraft coordinate system $\left(x_{i}, y_{i}, z_{i}\right)$ :

$$
\begin{aligned}
& x_{i}=x_{i}^{\prime \prime} \cos \delta_{y}-z_{i}^{\prime \prime} \sin \delta_{y} \\
& y_{i}=y_{i}^{\prime \prime} \\
& z_{i}=x_{i}^{\prime \prime} \sin \delta_{y}+z_{i}^{\prime \prime} \cos \delta_{y}
\end{aligned}
$$

The transformation from instrument coordinates to spacecraft coordinates can be written as:

$$
A_{\text {in } 2 s c}=\left[\begin{array}{ccc}
\cos \delta_{y} \cos \delta_{z}-\sin \delta_{y} \sin \delta_{x} \sin \delta_{z} & \cos \delta_{y} \sin \delta_{z}+\sin \delta_{y} \sin \delta_{x} \cos \delta_{z} & -\sin \delta_{y} \cos \delta_{x} \\
-\cos \delta_{x} \sin \delta_{z} & \cos \delta_{x} \cos \delta_{z} & \sin \delta_{x} \\
\sin \delta_{y} \cos \delta_{z}+\cos \delta_{y} \sin \delta_{x} \sin \delta_{z} & \sin \delta_{y} \sin \delta_{z}+\cos \delta_{y} \sin \delta_{x} \cos \delta_{z} & \cos \delta_{y} \cos \delta_{x}
\end{array}\right]
$$

Conversely. the transformation from spacecraft coordinates to instrument coordinates can be written as:

$$
A_{s c 2 i n}=\left[\begin{array}{ccc}
\cos \delta_{y} \cos \delta_{z}-\sin \delta_{y} \sin \delta_{x} \sin \delta_{z} & -\cos \delta_{x} \sin \delta_{z} & \sin \delta_{y} \cos \delta_{z}+\cos \delta_{y} \sin \delta_{x} \sin \delta_{z} \\
\cos \delta_{y} \sin \delta_{z}+\sin \delta_{y} \sin \delta_{x} \cos \delta_{z} & \cos \delta_{x} \cos \delta_{z} & \sin \delta_{y} \sin \delta_{z}+\cos \delta_{y} \sin \delta_{x} \cos \delta_{z} \\
-\sin \delta_{y} \cos \delta_{x} & \sin \delta_{x} & \cos \delta_{y} \cos \delta_{x}
\end{array}\right]
$$




\section{E.4 General Motion of the Spacecraft}

The general motion of the spacecraft is governed by the Euler Equations:

$$
\begin{aligned}
& T_{c x}+T_{d x}=I_{x x} \omega_{x}+\left(I_{z z}-I_{y y}\right) \omega_{y} \omega_{z} \\
& T_{c y}+T_{d y}=I_{y y} \omega_{y}+\left(I_{x x}-I_{z z}\right) \omega_{x} \omega_{z} \\
& T_{c z}+T_{d z}=I_{z z} \dot{\omega}_{z}+\left(I_{y y}-I_{x x}\right) \omega_{y} \omega_{x}
\end{aligned}
$$

\section{E.4.1 Free Motion of the Spacecraft}

Consider the torque free or polhode motion of the vehicle:

$$
\begin{aligned}
& 0=I_{x x} \dot{\omega}_{x}+\left(I_{z z}-I_{y y}\right) \omega_{y} \omega_{z} \\
& 0=I_{y y} \dot{\omega}_{y}+\left(I_{x x}-I_{z z}\right) \omega_{x} \omega_{z} \\
& 0=I_{z z} \omega_{z}+\left(I_{y y}-I_{x x}\right) \omega_{y} \omega_{x}
\end{aligned}
$$

This can be linearized by assuming small perturbations about a large roll velocity:

$$
\begin{aligned}
& \omega_{x}=\omega_{1} \\
& \omega_{y}=\omega_{2} \\
& \omega_{z}=\Omega+\omega_{3} \\
& 0=I_{x x} \cdot \omega_{1}+\left(I_{z z}-I_{y y}\right) \Omega \omega_{2} \\
& 0=I_{y y} \cdot \omega_{2}+\left(I_{x x}-I_{z z}\right) \Omega \omega_{1} \\
& 0=I_{z z} \omega_{3}
\end{aligned}
$$

This can be written in the LaPlace domain as:

$$
\left[\begin{array}{ccc}
I_{x x} s & \left(I_{z z}-I_{y y}\right) \mathrm{R} & 0 \\
\left(I_{x x}-I_{z}\right) \Omega & I_{y y} s & 0 \\
0 & 0 & I_{z z} s
\end{array}\right]\left\{\begin{array}{l}
\omega_{1} \\
\omega_{2} \\
\omega_{3}
\end{array}\right\}=\left\{\begin{array}{l}
0 \\
0 \\
0
\end{array}\right\}
$$


Note that the polhode motion of the satellite has been decoupled from the spinning motion about the z-axis. The general solution to these equations can be written as:

$$
\begin{aligned}
& \omega_{1}=\frac{\dot{\omega}_{1}(0)}{p} \sin (p t)+\omega_{1}(0) \cos (p t) \\
& \omega_{2}=\frac{\dot{\omega}_{2}(0)}{p} \sin (p t)+\omega_{2}(0) \cos (p t) \\
& \omega_{3}=\omega_{3}\left(t_{0}\right)
\end{aligned}
$$

where the polhode frequency, $p$, is:

$$
p^{2}=-\frac{\left(I_{z z}-I_{y y}\right)\left(I_{x x}-I_{z z}\right)}{I_{x x} I_{y y}} \Omega^{2}
$$

The angular velocity of the satellite can be written in terms of the euler rates in the body axes as:

$$
\begin{aligned}
& \omega_{x}=\dot{\psi} \cos \theta \sin \phi+\dot{\theta} \cos \phi \\
& \omega_{y}=\dot{\psi} \cos \theta \cos \phi-\dot{\phi} \sin \theta \\
& \omega_{z}=\dot{\cdot} \cdot \dot{\psi} \sin \theta
\end{aligned}
$$

Alternately, the euler rates can be written in terms of the angular velocity:

$$
\begin{aligned}
& \dot{\phi}=\omega_{z}+\left[\omega_{x} \sin \phi+\omega_{y} \cos \phi\right] \tan \theta \\
& \dot{\theta}=\omega_{x} \cos \phi-\omega_{y} \sin \phi \\
& \dot{\psi}=\frac{1}{\cos \theta}\left[\omega_{x} \sin \phi+\omega_{y} \cos \phi\right]
\end{aligned}
$$

For the case of a vehicle designed to spin about its largest principal moment of inertia. the motion of the spin axis will be restricted to motion near the $z$-axis. That is. $\psi$ and $\theta$ will be small. Furthermore, the roll rate can be assumed to be a perturbation about some large fixed rate, $\Omega$. The roll angle can also be assumed to be some small perturbation about an initial roll angle. $\phi_{0}$. The euier rate terms can then be rewritten as: 


$$
\begin{aligned}
& \dot{\phi}=\Omega+\omega_{3} \\
& \dot{\theta}=\omega_{1} \cos \phi_{0}-\omega_{2} \sin \phi_{0} \\
& \dot{\psi}=\omega_{1} \sin \phi_{0}+\omega_{2} \cos \phi_{0}
\end{aligned}
$$

These equations can be used to related the initial conditions between the euler angles and the angular velocity in body coordinates:

$$
\begin{aligned}
\omega_{1}(0)=\dot{\psi}(0) \sin \phi_{0}+\theta(0) \cos \phi_{0} \\
\dot{\omega_{1}(0)=} \dot{\psi}(0) \sin \phi_{0}+\dot{\theta}(0) \cos \phi_{0} \\
\omega_{2}(0)=\dot{\psi}(0) \cos \phi_{0}-\dot{\theta}(0) \sin \phi_{0} \\
\dot{\cdot} \cdot \dot{\cdot}(0) \cos \phi_{0}-\dot{\theta}(0) \sin \phi_{0} \\
\omega_{2}(0)=\dot{\psi}(0) \\
\omega_{3}(0)=\dot{\phi}(0)-\Omega
\end{aligned}
$$

The differential equations for $\theta$ and $\psi$ are first order linear differential equations that are coupled by the forcing terms $\omega_{1}$ and $\omega_{2}$. The roll angle. $\phi$, is described by a single first order ordinary differential equation. Note that the polhode motion described by pitch and yaw is uncoupled from the rolling motion about the z-axis. The general form of their solutions will be:

$$
\begin{gathered}
\theta(t)=\theta(0)+\left(\frac{\omega_{1}(0)}{p^{2}} \cos \phi_{0}-\frac{\omega_{2}(0)}{p^{2}} \sin \phi_{0}\right)(1-\cos (p t))+ \\
\left(\frac{\omega_{1}(0)}{p} \cos \phi_{0}-\frac{\omega_{2}(0)}{p} \sin \phi_{0}\right) \sin (p t) \\
\psi(t)=\psi(0)+\left(\frac{\omega_{1}(0)}{p^{2}} \sin \phi_{0}+\frac{\omega_{2}(0)}{p^{2}} \cos \phi_{0}\right)(1-\cos (p t))+ \\
\left(\frac{\omega_{1}(0)}{p} \sin \phi_{0}+\frac{\omega_{2}(0)}{p} \cos \phi_{0}\right) \sin (p t) \\
\phi(t)=\phi(0)+\Omega
\end{gathered}
$$


Equations E.4.10 can be used to simplify Equation E.4.11, viz.:

$$
\begin{aligned}
& \theta(t)=\theta(0)+\frac{\theta(0)}{p^{2}}(1-\cos (p t))+\frac{\theta(0)}{p} \sin (p t) \\
& \psi(t)=\psi(0)+\frac{\psi(0)}{p^{2}}(1-\cos (p t))+\frac{\psi(0)}{p} \sin (p t) \\
& \phi(t)=\phi(0)+\Omega t
\end{aligned}
$$

\section{E.5 Guide Star Location in Instrument Frame}

By the definition of the Guide Star Coordinates. the location of the guide star can be written in Guide Star Coordinates as.

$$
\vec{R}_{g s}=\left[\begin{array}{lll}
0 & 1 & 0
\end{array}\right]\left\{\begin{array}{c}
\hat{X} \\
\dot{y} \\
Y \\
Z
\end{array}\right\}
$$

This can be transformed to the spacecraft body frame using the euler angles. viz.:

$$
\bar{R}_{g s}=\left[\begin{array}{c}
\cos (\theta) \sin (\phi) \\
\cos (\theta) \cos (\phi) \\
-\sin (\theta)
\end{array}\right]^{T}\left\{\begin{array}{c}
x \\
x \\
y \\
z
\end{array}\right\}
$$

In general. the instrument frame will not coincide exactly with the spacecraft frame. Using the transformation E.2.5 from above. the guide star location can be written in instrument coordinates as.

$$
\vec{R}_{g s}=\left[\begin{array}{c}
\cos (\theta) \sin (\phi) \\
\cos (\theta) \cos (\phi) \\
-\sin (\theta)
\end{array}\right]^{T} A_{s c 2 i n}\left\{\begin{array}{c}
\hat{x}_{i} \\
\hat{y}_{i} \\
\hat{z}_{i}
\end{array}\right\}
$$

In Appendix D. the two angles that define the transmission of the collimator were denoted $\alpha$ and $\rho$. The guide star vector can be written in terms of $\alpha$ and $\rho$. viz.: 


$$
\vec{R}_{g s}=\left[\begin{array}{c}
-\frac{\tan (\rho)}{\sqrt{1+\tan ^{2}(\alpha)+\tan ^{2}(\rho)}} \\
\frac{1}{\sqrt{1+\tan ^{2}(\alpha)+\tan ^{2}(\rho)}}
\end{array}\right]^{T}\left\{\begin{array}{c}
\frac{\tan (\alpha)}{\sqrt{1+\tan ^{2}(\alpha)+\tan ^{2}(\rho)}} \\
\hat{y}_{i} \\
\hat{z}_{i}
\end{array}\right\}
$$

Equating the three terms in E.5.3 and E.5.4, we can solve for $\alpha$ and $\rho$ in terms of both the known spacecraft attitude $(\phi, \theta, \psi)$ and the orientation of the instrument in the spacecraft $\left(\delta_{x}, \delta_{y}, \delta_{z}\right)$. If this were to be done for a general case. we would have to solve this numerically. If, however. we assume that the instrument axes are close to the spacecraft axes and that the pitch and yaw euler angles are small and roll is small about some initial roll angle $\phi_{0}=0$ (by definition of the guide star location), we can simplify the problem by making small angle approximations, viz.:

$$
\begin{aligned}
& {\left[\begin{array}{c}
-\rho \\
1 \\
\alpha
\end{array}\right]^{T}=\left[\begin{array}{c}
\phi \\
1 \\
-\theta
\end{array}\right]^{T}\left[\begin{array}{ccc}
1 & -\delta_{z} & \delta_{y} \\
\delta_{z} & 1 & -\delta_{x} \\
-\delta_{y} & \delta_{x} & 1
\end{array}\right]} \\
& {\left[\begin{array}{c}
-\rho \\
1 \\
\alpha
\end{array}\right]^{T}=\left[\begin{array}{c}
\phi+\delta_{z} \\
1 \\
-\delta_{x}-\theta
\end{array}\right]^{r}}
\end{aligned}
$$

Or.

$$
\begin{aligned}
& \rho=-\left(\phi+\delta_{z}\right) \\
& \alpha=-\left(\theta+\delta_{x}\right)
\end{aligned}
$$




\section{E.6 Motion of Guide Star in Instrument Frame}

We can combine E.5.6 with E.4.12 to get an expression for the motion of the guide star in instrument coordinates, viz:

$$
\begin{aligned}
& \rho=-\left(\phi(0)+\Omega t+\delta_{z}\right) \\
& \alpha \cdot \\
& \alpha=-\left(\theta(0)+\frac{\theta(0)}{p^{2}}(1-\cos (p t))+\frac{\theta(0)}{p} \sin (p t)+\delta_{x}\right)
\end{aligned}
$$

The initial roll angle $\phi(0)$ has been previously defined to be 0 . If the polhode frequency, $p$, is small compared to the nominal roll rate. $\Omega$. then pt will be second order small. Equation E.6.1 can be simplified, viz.:

$$
\begin{aligned}
& \rho=-\left(\Omega t+\delta_{z}\right) \\
& \alpha=-\left(\theta(0)+\delta_{x}\right)
\end{aligned}
$$




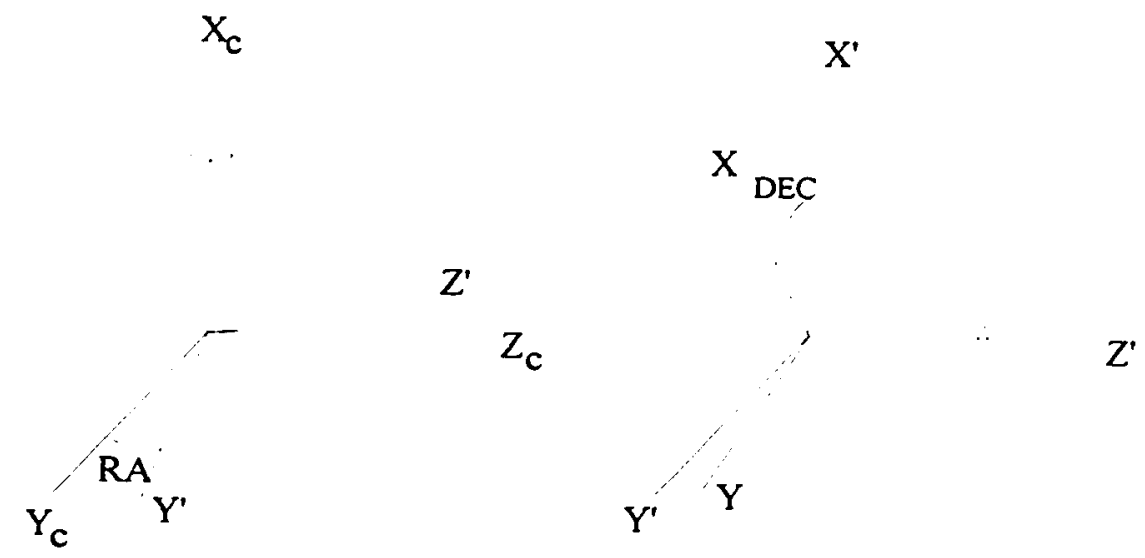

Figure E.1: Transformation from Celestial to Guide Star Coordinates

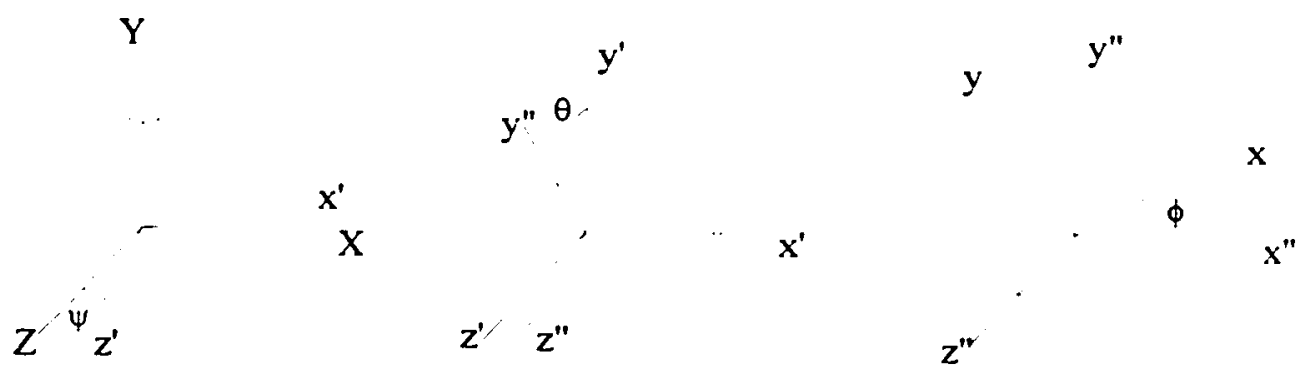

Figure E.2: Transformation from Guide Star to Spacecraft Coordinates 


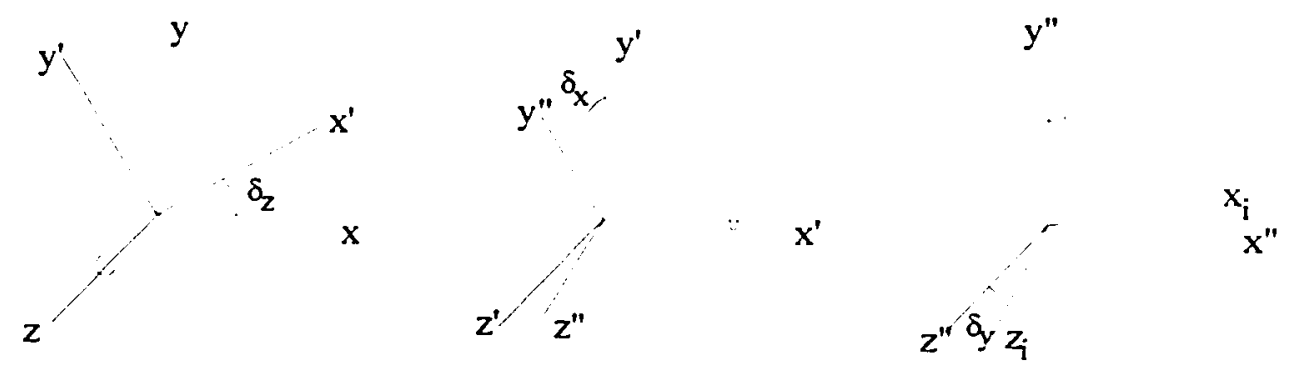

Figure E.3: Transformation from Spacecraft to Instrument Coordinates

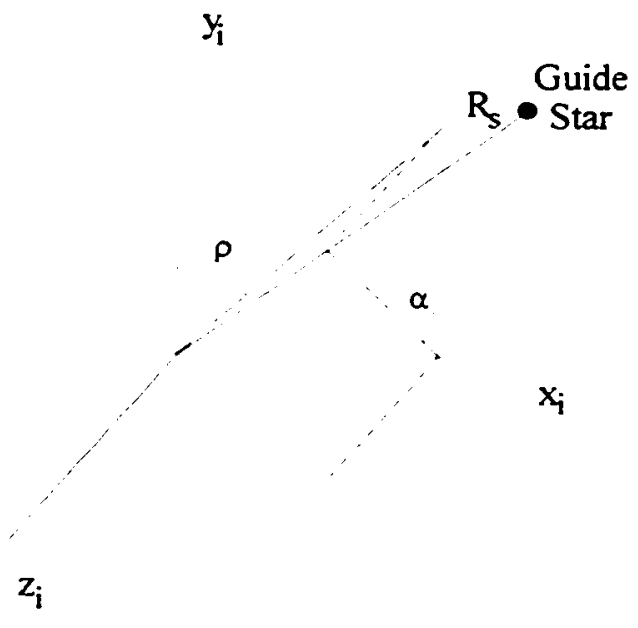

Figure E.4: Guide Star Location in Instrument Coordinates 


\section{Appendix F: Second Order Star Scanner Model}

\section{F.1 Introduction}

In Appendix $E$ it was shown that the operation of an $x$-ray star scanner can be described by a model that neglects any terms that are second order small and smaller. However. it may be helpful to include some of these terms. This may lead to improvements in the performance of the $x$-ray star scanner. In this Appendix. a second order star scanner model will be created by including the quadratic terms neglected in Appendix E. The derivation of this model will directly parallel the derivation of the first order model. using the same coordinate systems and terms.

\section{F.2 Representation of Instrument Misalignments}

The misalignment of the instrument in spacecraft coordinates can be modeled by assuming that the euler rotations that represent the instrument axes in the spacecraft frame $\left(\delta_{x}, \delta_{y}, \delta_{z}\right)$ have small errors associated with them. The actual euler rotations are 
then $\left(\delta_{x}+\varepsilon_{x}, \delta_{y}+\varepsilon_{y}, \delta_{z}+\varepsilon_{z}\right)$. Equation E.3.5 can be rewritten in terms of these new rotations, viz.:

$$
\begin{aligned}
& A_{s c 2 i n}\left({ }^{*}, 1\right)=\left[\begin{array}{c}
\cos \left(\delta_{y}+\varepsilon_{y}\right) \cos \left(\delta_{z}+\varepsilon_{z}\right)-\sin \left(\delta_{y}+\varepsilon_{y}\right) \sin \left(\delta_{x}+\varepsilon_{x}\right) \sin \left(\delta_{z}+\varepsilon_{z}\right) \\
\cos \left(\delta_{y}+\varepsilon_{y}\right) \sin \left(\delta_{z}+\varepsilon_{z}\right)+\sin \left(\delta_{y}+\varepsilon_{y}\right) \sin \left(\delta_{x}+\varepsilon_{x}\right) \cos \left(\delta_{z}+\varepsilon_{z}\right) \\
-\sin \left(\delta_{y}+\varepsilon_{y}\right) \cos \left(\delta_{x}+\varepsilon_{x}\right)
\end{array}\right] \\
& A_{s c 2 i n}\left({ }^{*} .2\right)=\left[\begin{array}{c}
-\cos \left(\delta_{x}+\varepsilon_{x}\right) \sin \left(\delta_{z}+\varepsilon_{z}\right) \\
\cos \left(\delta_{x}+\varepsilon_{x}\right) \cos \left(\delta_{z}+\varepsilon_{z}\right) \\
\sin \left(\delta_{x}+\varepsilon_{x}\right)
\end{array}\right] \\
& A_{s c 2 \text { in }}\left({ }^{*}, 3\right)=\left[\begin{array}{c}
\sin \left(\delta_{y}+\varepsilon_{y}\right) \cos \left(\delta_{z}+\varepsilon_{z}\right)+\cos \left(\delta_{y}+\varepsilon_{y}\right) \sin \left(\delta_{x}+\varepsilon_{y}\right) \sin \left(\delta_{z}\right) \\
\sin \left(\delta_{y}+\varepsilon_{y}\right) \sin \left(\delta_{z}+\varepsilon_{z}\right)+\cos \left(\delta_{y}+\varepsilon_{y}\right) \sin \left(\delta_{x}+\varepsilon_{x}\right) \cos \left(\delta_{z}+\varepsilon_{z}\right) \\
\cos \left(\delta_{y}+\varepsilon_{y}\right) \cos \left(\delta_{x}+\varepsilon_{x}\right)
\end{array}\right]
\end{aligned}
$$

Expanding the terms in F.2.1 by Taylor Series expansion yields a quadratic approximation to $A_{\mathrm{sc} 2 \mathrm{in}}$, viz.:

$$
A_{s c \text { in }}=\left[\begin{array}{ccc}
1+\frac{\left(\delta_{y}+\varepsilon_{y}\right)^{2}}{2}+\frac{\left(\delta_{z}+\varepsilon_{z}\right)^{2}}{2} & -\left(\delta_{z}+\varepsilon_{z}\right) & \left(\delta_{y}+\varepsilon_{y}\right)+\left(\delta_{x}+\varepsilon_{x}\right)\left(\delta_{z}+\varepsilon_{z}\right) \\
\left(\delta_{z}+\varepsilon_{z}\right)+\left(\delta_{x}+\varepsilon_{x}\right)\left(\delta_{y}+\varepsilon_{y}\right) & 1+\frac{\left(\delta_{x}+\varepsilon_{x}\right)^{2}}{2}+\frac{\left(\delta_{z}+\varepsilon_{z}\right)^{2}}{2} & \left(\delta_{x}+\varepsilon_{x}\right)+\left(\delta_{y}+\varepsilon_{y}\right)\left(\delta_{z}+\varepsilon_{z}\right) \\
-\left(\delta_{y}+\varepsilon_{y}\right) & \left(\delta_{x}+\varepsilon_{x}\right) & 1+\frac{\left(\delta_{x}+\varepsilon_{x}\right)^{2}}{2}+\frac{\left(\delta_{y}+\varepsilon_{y}\right)^{2}}{2}
\end{array}\right]
$$

\section{F.3 Free Motion of the Spacecraft}

The torque free motion of the vehicle can be represented by the euler equations. viz.:

$$
\begin{aligned}
& 0=I_{x x} \dot{\omega}_{x}+\left(I_{z z}-I_{z y}\right) \omega_{y} \omega_{z} \\
& 0=I_{y y} \dot{\omega}_{y}+\left(I_{x x}-I_{z z}\right) \omega_{x} \omega_{z} \\
& 0=I_{z z} \dot{\omega}_{z}+\left(I_{y y}-I_{x x}\right) \omega_{y} \omega_{x}
\end{aligned}
$$


In this analysis, we will assume that the vehicle is nearly a body of rotation. viz.:

$$
\begin{aligned}
& I_{x x}=I_{t} \\
& I_{y y}=I_{t}+\delta I \\
& I_{z z}=I_{a}
\end{aligned}
$$

Again assuming that the vehicle is primarily spinning about the z-axis. the euler equations can be reduced to a quadratic model, viz:

$$
\begin{aligned}
& \omega_{x}=\omega_{1} \\
& \omega_{y}=\omega_{2} \\
& \omega_{z}=\Omega+\omega_{3} \\
& 0=I_{t} \dot{\omega}_{1}+\left(I_{a}-I_{t}-\delta I\right) \Omega \omega_{2} \\
& 0=\left(I_{t}+\delta I\right) \dot{\omega}_{2}+\left(I_{t}-I_{a}\right) \Omega \omega_{1} \\
& 0=I_{a} \dot{\omega}_{3}
\end{aligned}
$$

This can be written in the LaPlace domain as:

$$
\left[\begin{array}{ccc}
I_{t} s & \left(I_{a}-I_{t}-\delta I\right) \Omega & 0 \\
\left(I_{t}-I_{a}\right) \Omega & \left(I_{t}+\delta I\right) s & 0 \\
0 & 0 & I_{a} s
\end{array}\right]\left\{\begin{array}{l}
\omega_{1} \\
\omega_{2} \\
\omega_{3}
\end{array}\right\}=\left\{\begin{array}{l}
0 \\
0 \\
0
\end{array}\right\}
$$

Note that the polhode motion of the satellite has been decoupled from the spinning motion about the z-axis. The general solution to these equations can be written as:

$$
\begin{aligned}
& \omega_{1}=\frac{\dot{\omega}_{1}(0)}{p} \sin (p t)+\omega_{1}(0) \cos (p t) \\
& \omega_{2}=\frac{\dot{\omega}_{2}(0)}{p} \sin (p t)+\omega_{2}(0) \cos (p t) \\
& \omega_{3}=0
\end{aligned}
$$

where the polhode frequency. $\mathrm{p}$, is:

$$
p^{2}=-\frac{\left(I_{a}-I_{t}-\delta I\right)\left(I_{t}-I_{a}\right)}{I_{t}\left(I_{a}+\delta I\right)} \Omega^{2}
$$


Again, assuming that pt is small. F.3.6 can be reduced by neglecting terms smaller than second order, viz:

$$
\begin{aligned}
& \omega_{x}=\dot{\omega}_{1}(0) t+\omega_{1}(0) \\
& \omega_{y}=\dot{\omega}_{2}(0) t+\omega_{2}(0) \\
& \omega_{z}=\Omega
\end{aligned}
$$

From E.4.8, the euler rates can be written in terms of the angular velocity in the body axes, viz.:

$$
\begin{aligned}
& \dot{\phi}=\omega_{z}+\left[\omega_{x} \sin \phi+\omega_{y} \cos \phi\right] \tan \theta \\
& \dot{\theta}=\omega_{x} \cos \phi-\omega_{y} \sin \phi \\
& \dot{\psi}=\frac{1}{\cos \theta}\left[\omega_{x} \sin \phi+\omega_{y} \cos \phi\right]
\end{aligned}
$$

For the case of a vehicle designed to spin about its axis of largest principal moment of inertia, the motion of the spin axis will be restricted to motion near the z-axis. That is. $y^{\prime}$ and $\theta$ will be small. Furthermore, the roll rate can be assumed to be a perturbation about some large fixed rate. $\Omega$. The roll angle can also be assumed to be some small perturbation about an initial roll angle. $\phi_{0}$. But by the definition of the guide star coordinate system, $\phi_{0}$ is zero. Keeping the quadratic terms. the euler rate terms can then be rewritten as:

$$
\begin{aligned}
& \dot{\phi}=\Omega+\omega_{2} \theta \\
& \dot{\theta}=\omega_{1}-\omega_{2} \phi \\
& \dot{\psi}=\omega_{2}+\omega_{1} \phi
\end{aligned}
$$

Combining F.3.9 and F.3.7, we get the differential equations describing the polhode motion of the spinning vehicle to second order. viz.:

$$
\begin{aligned}
& \dot{\phi}-\omega_{2}(0) \theta=\Omega \\
& \dot{\theta}+\omega_{2}(0) \phi=\omega_{1}(0) t+\omega_{1}(0) \\
& \dot{\psi}=\omega_{2}+\omega_{1} \phi
\end{aligned}
$$


The general solution for these coupled equations can be written as.

$$
\begin{aligned}
& \phi=\Omega t \\
& \theta=\theta(0)+\dot{\theta}(0) t \\
& \psi=\psi(0)+\dot{\psi}(0) t-\Omega \theta(0) t
\end{aligned}
$$

\section{F.4 Guide Star Location in Instrument Frame}

The location of the guide star can be written in spacecraft coordinates by E.4.2:

$$
\bar{R}_{g s}=\left[\begin{array}{c}
\cos (\theta) \sin (\phi) \\
\cos (\theta) \cos (\phi) \\
-\sin (\theta)
\end{array}\right]^{T}\left\{\begin{array}{c}
\hat{x} \\
\bar{y} \\
z
\end{array}\right\}
$$

This can be expanded by Taylor Series expansion, keeping the second order small terms, viz:

$$
\vec{R}_{g s}=\left[\begin{array}{c}
\phi \\
1+\frac{\theta^{2}}{2} 1+\frac{\phi^{2}}{2} \\
-\theta
\end{array}\right]^{T}\left\{\begin{array}{c}
\hat{x} \\
y \\
y \\
z
\end{array}\right\}
$$

The guide star location can be written in instrument coordinates, viz.:

$$
\bar{R}_{g s}=\left[\begin{array}{c}
\phi \\
1+\frac{\theta^{2}}{2} 1+\frac{\phi^{2}}{2} \\
-\theta
\end{array}\right]^{T} A_{s c 2 i n}\left\{\begin{array}{c}
\hat{x}_{i} \\
y_{i} \\
z_{i}
\end{array}\right\}
$$


Recalling equation E.4.5. this can also be written in terms of the collimator roll and itch angles, $\rho$ and $\alpha$. viz:

$$
\bar{R}_{g s}=\left[\begin{array}{c}
-\frac{\tan (\rho)}{\sqrt{1+\tan ^{2}(\alpha)+\tan ^{2}(\rho)}} \\
\frac{1}{\sqrt{1+\tan ^{2}(\alpha)+\tan ^{2}(\rho)}} \\
\frac{\tan (\alpha)}{\sqrt{1+\tan ^{2}(\alpha)+\tan ^{2}(\rho)}}
\end{array}\right]^{T}\left\{\begin{array}{c}
\hat{x}_{i} \\
\hat{y}_{i} \\
z_{i}
\end{array}\right\}
$$

Substituting F.2.2 into F.4.3 and equating the result to F.4.4. we can write the collimator roll and pitch angles in terms of modelable parameters, viz:

$$
\begin{aligned}
& \rho=-\left(\phi+\left(\delta_{x}-\theta\right) \varepsilon_{y}+\delta_{z}+\varepsilon_{z}+\varepsilon_{y} \varepsilon_{x}\right) \\
& \alpha=-\left(\theta+\varepsilon_{y}\left(\phi+\varepsilon_{z}\right)+\delta_{x}+\varepsilon_{x}\right)
\end{aligned}
$$

Substituting for the motion of the spacecraft as given by F.3.11 into F.4.5. we can describe the motion of the guide star in instrument coordinates. viz.:

$$
\begin{aligned}
& \rho=-\left(\Omega t+\delta_{z}+\varepsilon_{z}+\left(\theta(0)+\delta_{x}+\varepsilon_{x}\right)\left(\delta_{y}+\varepsilon_{y}\right)\right) \\
& \alpha=-\left(\theta(0)+\delta_{x}+\varepsilon_{x}\right)-\theta(0) t+\left(\varepsilon_{y}+\delta_{y}\right)\left(\Omega t+\delta_{z}+\varepsilon_{z}\right)
\end{aligned}
$$

Noting that two of the misalignment terms. $\varepsilon_{\mathrm{x}}$ and $\varepsilon_{\mathrm{z}}$. are unobservable. the motion of the guide star can be modeled as.

$$
\begin{aligned}
& \rho=-\left(\Omega t+\delta_{z}+\left(\theta(0)+\delta_{x}\right)\left(\delta_{y}+\varepsilon_{y}\right)\right) \\
& \left.\alpha=-\left(\theta(0)+\delta_{x}\right)-\theta(0) t+\left(\varepsilon_{y}+\delta_{y}\right)\left(\Omega t+\delta_{z}\right)\right)
\end{aligned}
$$




\section{Appendix G: HEAO A1 Studies - IDL Procedures}

\section{G.1 General Files}

function ASP2INST, Yr, Zr, Rr

: Convert from RA/DEC to Vectors

$\mathrm{Y}=$ transpose $(\operatorname{rd} 2 \mathrm{v}(\mathrm{Yr}(0), \mathrm{Yr}(1)))$

$Z=$ transpose $(\operatorname{rd} 2 v(\operatorname{Zr}(0), \operatorname{Zr}(1)))$

$R=$ transpose $(r d 2 v(\operatorname{Rr}(0) \cdot \operatorname{Rr}(1)))$

$\mathrm{x}=\operatorname{vecx}(\mathrm{y}, \mathrm{z})$

$\mathrm{L}=\mathrm{db} \operatorname{larr}(3.3)$

$\mathrm{L}(0, *)=\mathrm{x}$

$\mathrm{L}(1, *)=\mathrm{y}$

$L\left(2 .^{*}\right)=z$

rsi $=$ transpose $(r) \div$ transpose $(L)$

alpha $=\operatorname{asin}(r s i(2))$

beta $=\operatorname{atan}(r s i(0) .-r s i(1))$

$\operatorname{ran}=[$ beta,alpha $]$

retum, ran

end

FUNCTION RD2V. RA.DEC

$N=n \_$elements(RA)

$r=$ dblarr(N.3)

$r(* .0)=\cos (D E C) * \cos (R A)$

$r(*, 1)=\cos (\mathrm{DEC}) * \sin (\mathrm{RA})$

$r(2)=\sin (D E C)$

return. $r$

end 


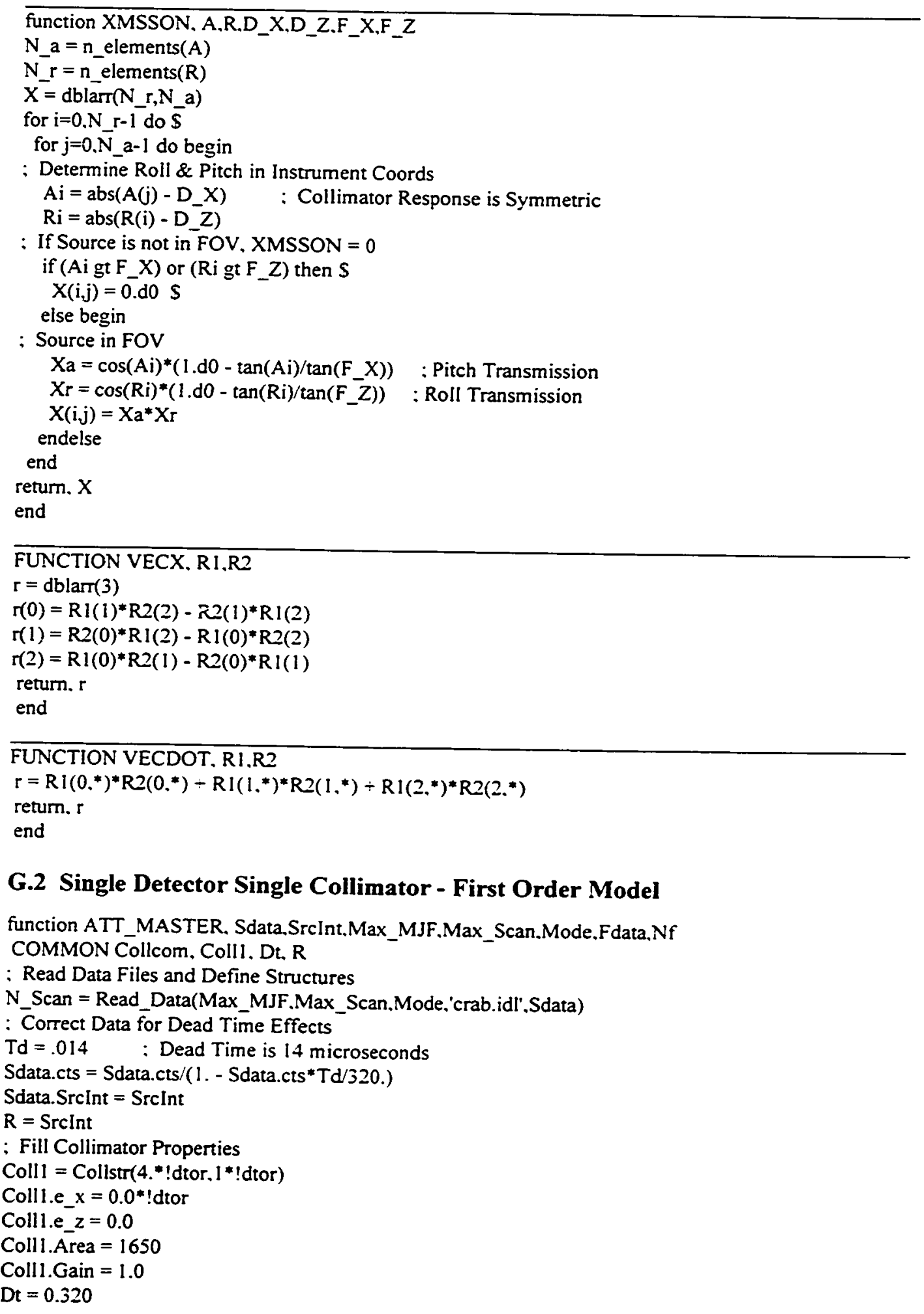




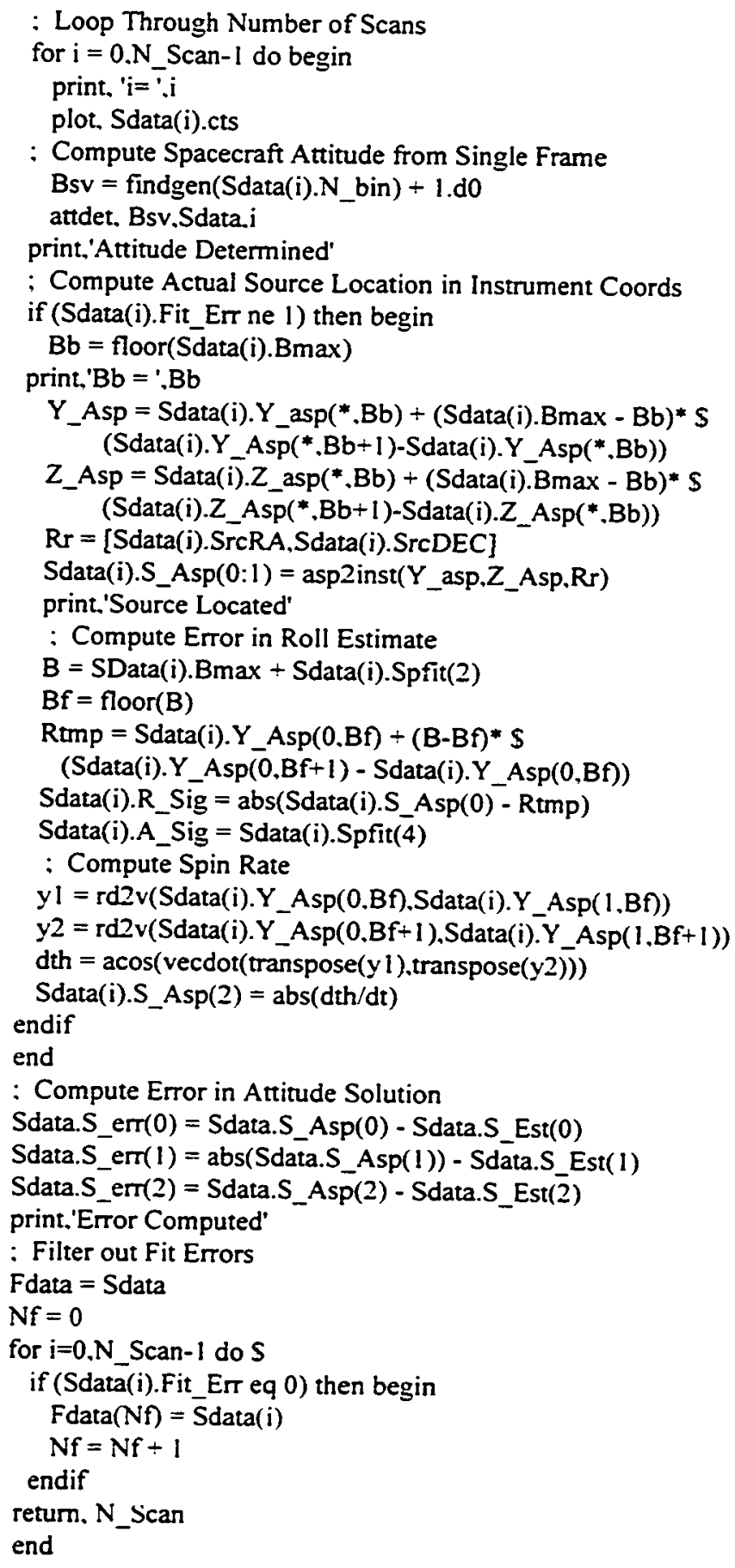




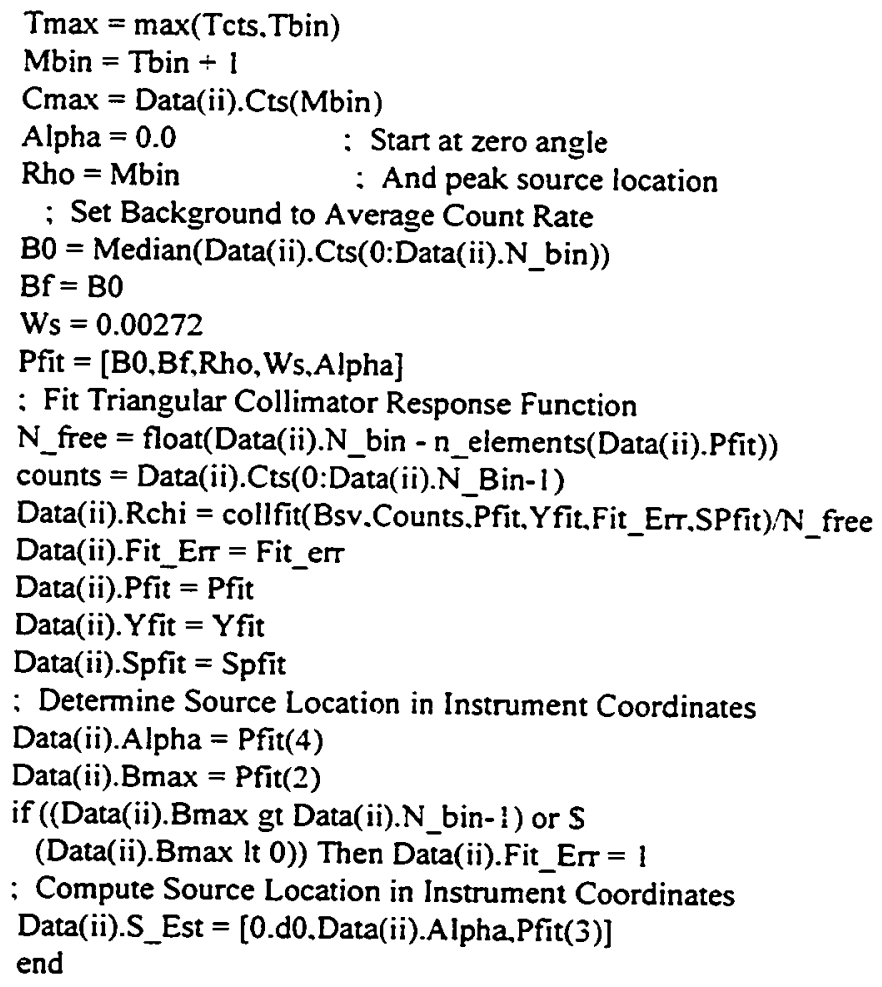




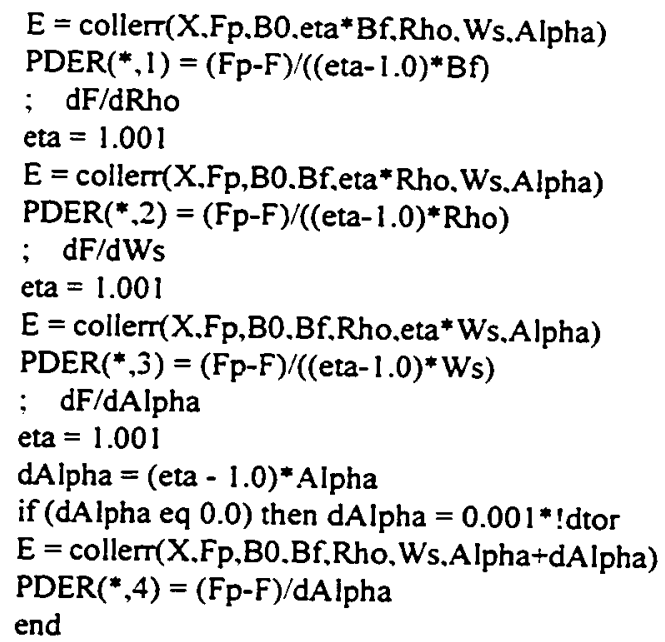



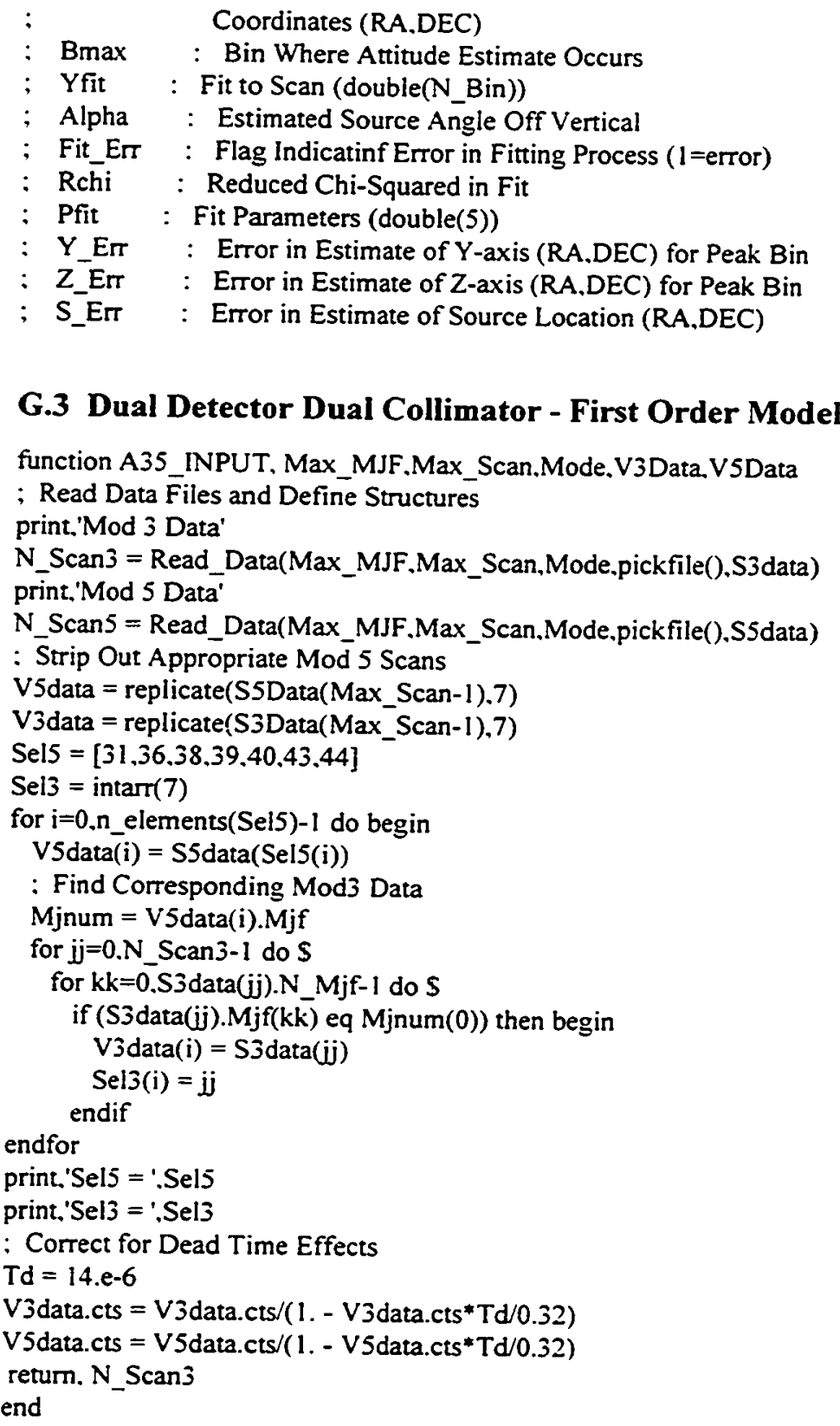

pro A35_Mast, X3data,X5data,N

COMMON COLLCOM. Coll1.Coll2,Dt

; Define Collimator Structures

: Collimator 1 - Mod 3

Coll1 = Collstr(8.* !dtor.2. ${ }^{*}$ !dtor)

Colll.e_ $x=0.0^{*}$ !dtor

Colli.e $z=0.0$

Colll . area $=1650$.

Coll 1 gain $=1.0$ 


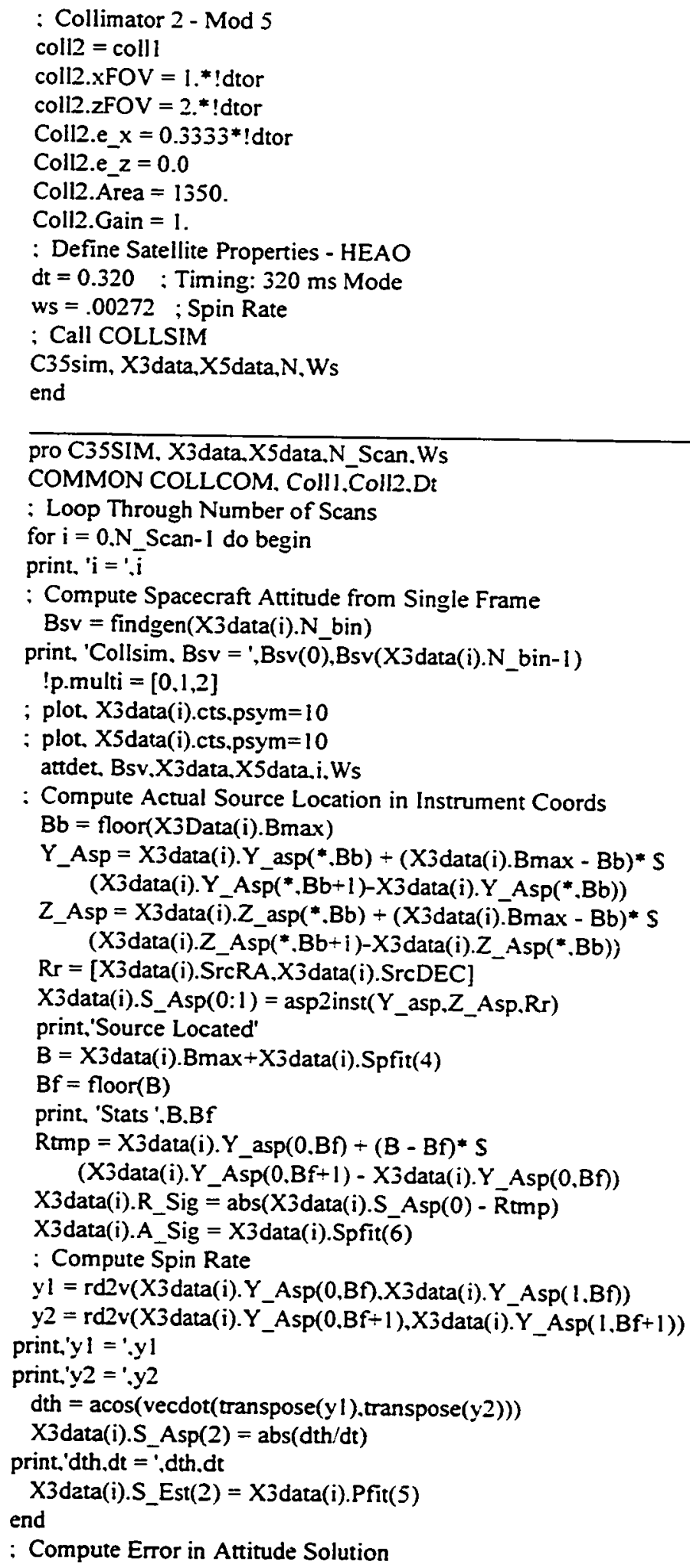


X3data.S_err $=$ X3data.S_Asp - X3̈data.S_Est

print.'Error Computed'

end

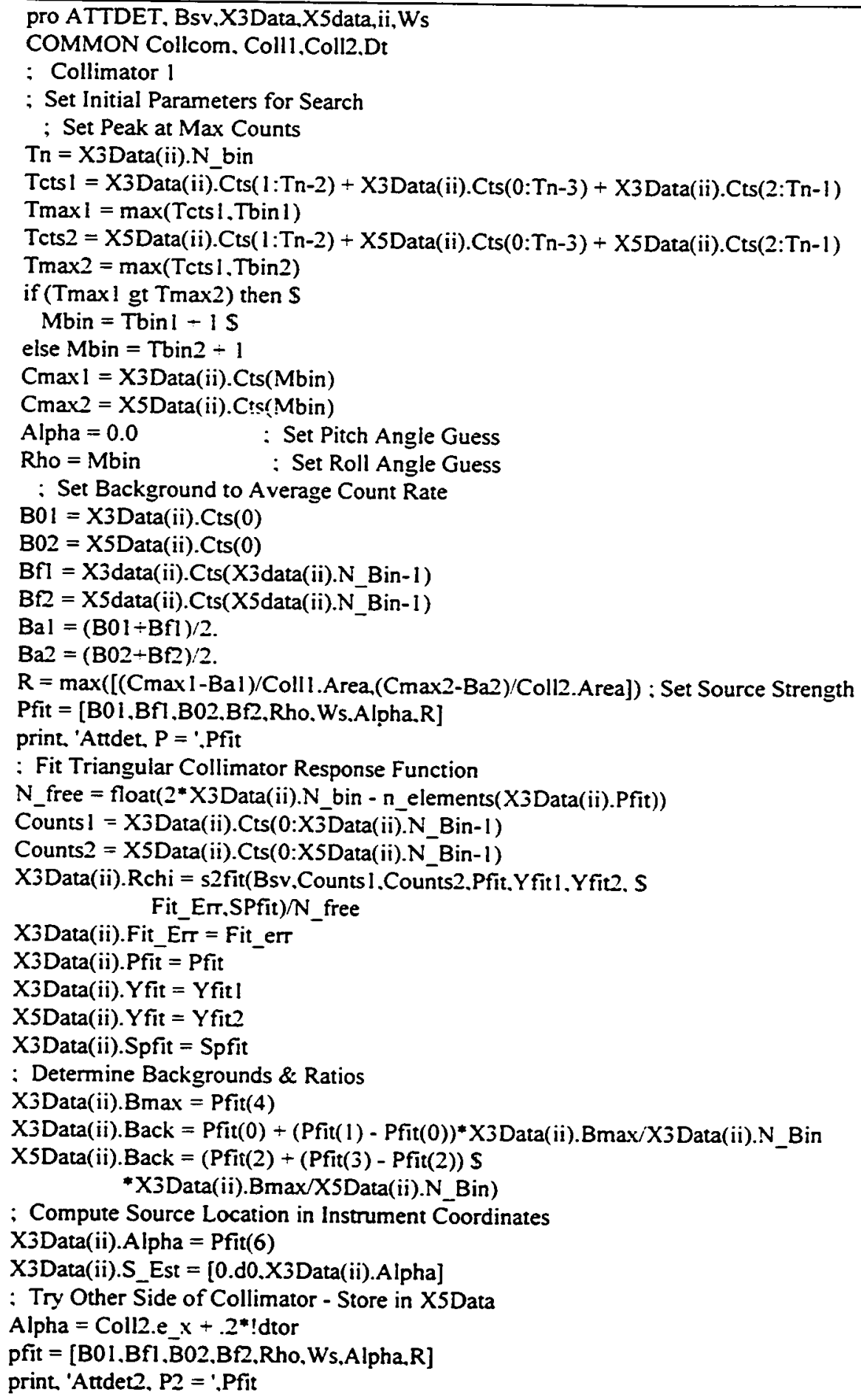




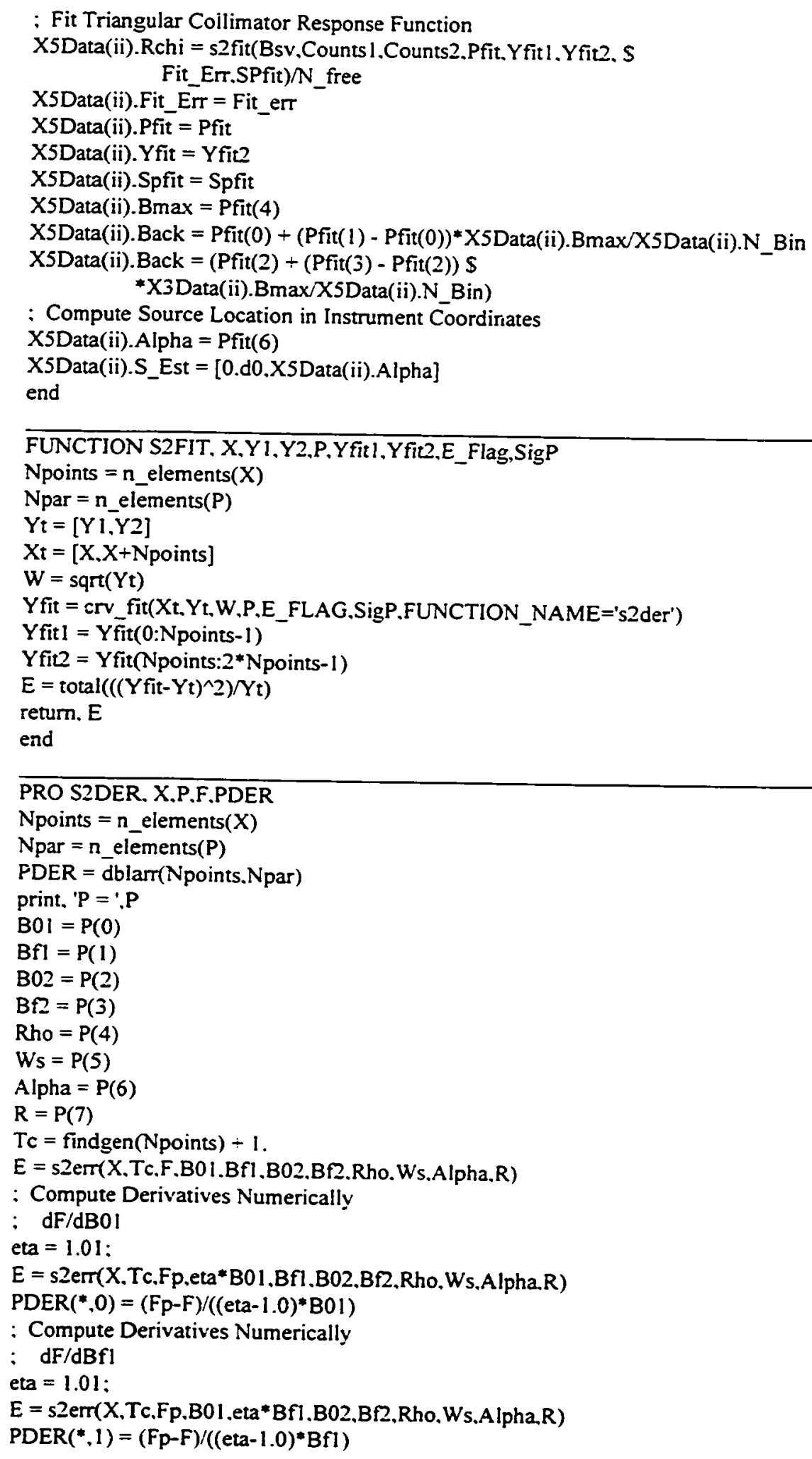




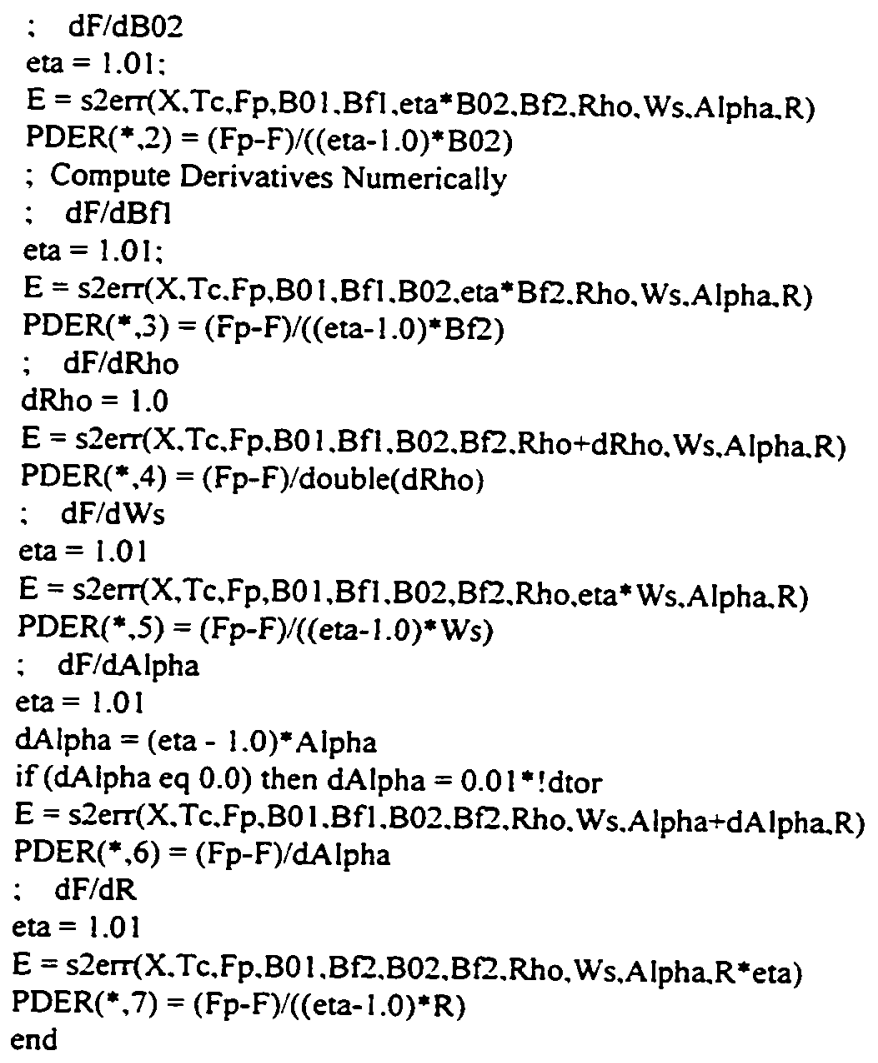


case 1 of

(X(i) It L1): begin : First Scan, To ist Break

$\operatorname{Trn}(\mathrm{i})=\mathrm{B} 01+(\mathrm{Bfl}-\mathrm{B} 0 \mathrm{l})^{*} \mathrm{X}(\mathrm{i}) /(\mathrm{N}-\mathrm{l})$ end

(X(i) ge $\mathrm{Ll}$ ) and (X(i) lt Rho): begin ; First Scan, Ist Side

$\mathrm{B}=\mathrm{B} 01+(\mathrm{Bfl}-\mathrm{B} 0 \mathrm{l}) * \mathrm{X}(\mathrm{i}) /(\mathrm{N}-\mathrm{l})$

$\operatorname{Tm}(i)=B+2^{*}(X(i)-L 1)^{*}(P 1) /(L 2-L 1)$

end

( $X(\mathrm{i})$ ge $\mathrm{Rho}$ ) and (X(i) It L2): begin : First Scan. 2nd Side

$\mathrm{B}=\mathrm{B} 0 \mathrm{l}+(\mathrm{BFl}-\mathrm{B} 0 \mathrm{l}) * \mathrm{X}(\mathrm{i}) /(\mathrm{N}-\mathrm{l})$

$\operatorname{Tm}(\mathrm{i})=\mathrm{B}+2^{*}(\mathrm{~L} 2-\mathrm{X}(\mathrm{i}))^{*}(\mathrm{P} 1) /(\mathrm{L} 2-\mathrm{L} 1)$

end

(X(i) ge $L 2)$ and $(X(i)$ It $N)$ : begin ; First Scan. From 2nd Side

$\mathrm{B}=\mathrm{B} 0 \mathrm{l}+(\mathrm{Bfl}-\mathrm{B} 0 \mathrm{l}) * \mathrm{X}(\mathrm{i}) /(\mathrm{N}-\mathrm{l})$

$\operatorname{Tm}(i)=B$

end

( $X(\mathrm{i})$ ge $N$ ) and $(X(i)$ it $N+L 3$ ): begin : 2nd Scan. To ist Side

$\operatorname{Tr}(\mathrm{i})=(\mathrm{B} 02+(\mathrm{Bf} 2-\mathrm{B} 02) *(\mathrm{X}(\mathrm{i})-\mathrm{N}) /(\mathrm{N}-\mathrm{I}))$ end

(X(i) ge $N+L 3$ ) and (X(i) it $N+R h o)$ : begin : 2nd Scan. Ist Side

$\mathrm{B}=(\mathrm{B} 02+(\mathrm{B} 2-\mathrm{B} 02) *(\mathrm{X}(\mathrm{i})-\mathrm{N}) /(\mathrm{N}-1))$

$\operatorname{Trn}(\mathrm{i})=\mathrm{B}+2^{*}(\mathrm{X}(\mathrm{i})-\mathrm{N}-\mathrm{L} 3)^{*}(\mathrm{P} 2) /(\mathrm{L} 4-\mathrm{L} 3)$

end

( $X(i)$ ge $N+R h o$ ) and (X(i) it $N+L 4)$ : begin : 2nd Scan. 2nd Side

$\mathrm{B}=\left(\mathrm{B} 02+(\mathrm{Bf} 2-\mathrm{B} 02)^{*}(\mathrm{X}(\mathrm{i})-\mathrm{N}) /(\mathrm{N}-1)\right)$

$\operatorname{Tm}(i)=B+2 *(L 4-X(i)+N) *(P 2) /(L 4-L 3)$ end

(X(i) ge $N+L 4)$ and $(X(i)$ It $N$ t): begin : 2nd Scan. End

$B=\left(B 02 \div(B f 2-B 02)^{*}(X(i)-N) /(N-1)\right)$

$\operatorname{Tm}(\mathrm{i})=\mathrm{B}$

end

else: print. ' $X$ has illegal value: '.i.X(i)

endcase

$E=\operatorname{total}\left(\left((\mathrm{Cts}-\operatorname{Trn})^{\wedge} 2\right) / \mathrm{Cts}\right)$

return. $E$

end

\section{G.4 Dual Detector Dual Collimator - Second Order Model}

pro ATTDET, Bsv, X3Data,X5data.ii,Ws

COMMON Collcom, Colli,Coll2.Dt

: Collimator 1

: Set Initial Parameters for Search

: Set Peak at Max Counts

$\mathrm{Tn}=$ X3Data(ii).N_bin

Tcts $1=X 3$ Data(ii).Cts(1:Tn-2) + X3Data(ii).Cts(0:Tn-3) + X3Data(ii).Cts(2:Tn-1)

Tmax $1=\max ($ Tcts 1, Tbin 1$)$

Tcts2 = X5Data(ii).Cts(1:Tn-2) + X5Data(ii).Cts(0:Tn-3) + X5Data(ii).Cts(2:Tn-1)

$T \max 2=\max ($ Tcts 1, Tbin 2$)$

if (Tmaxl gt Tmax2) then $S$

Mbin $=$ Tbin $1+1 \mathrm{~S}$

else Mbin = Tbin $2+1$

Cmaxl $=$ X3Data(ii).Cts(Mbin) 


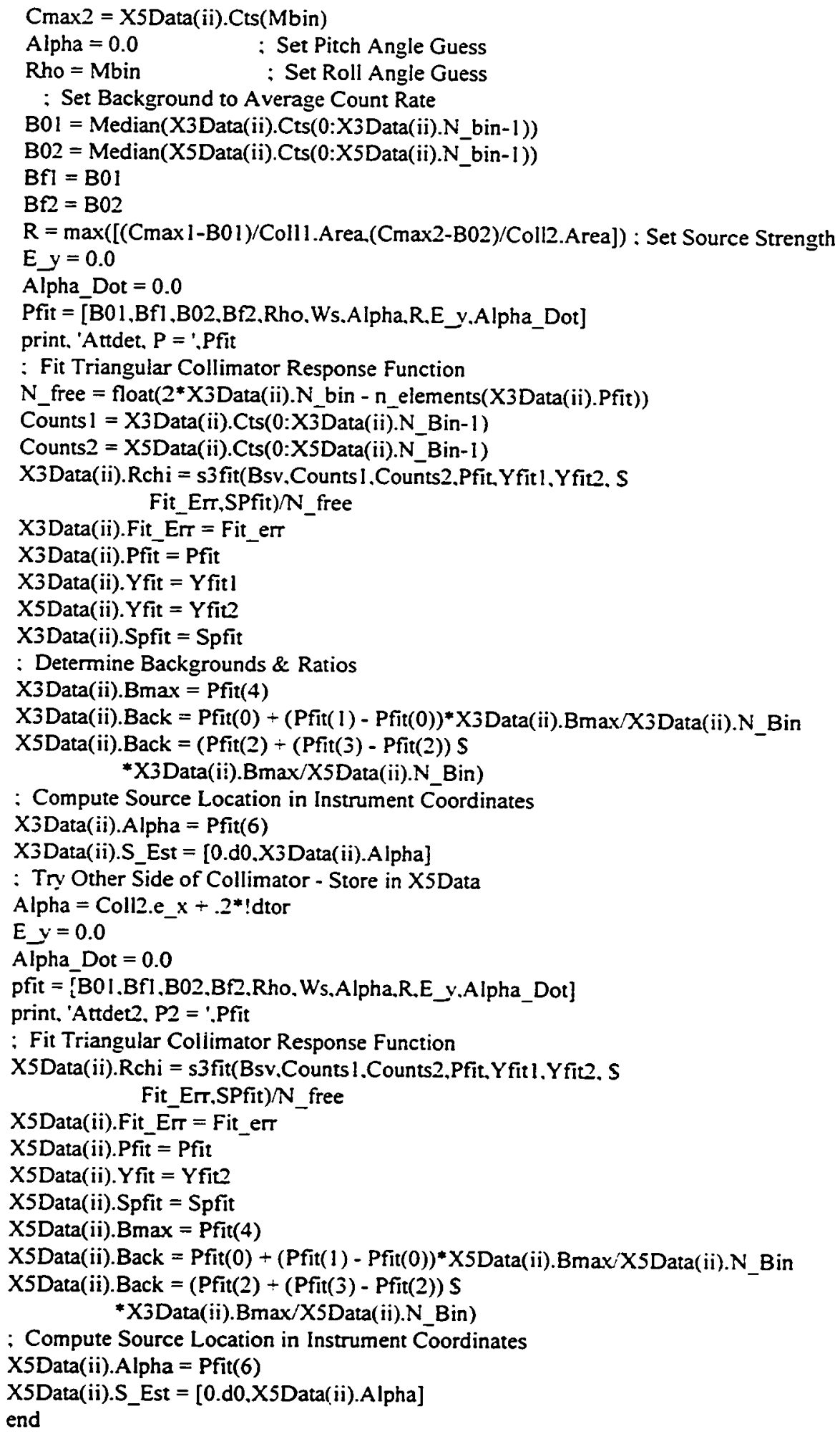




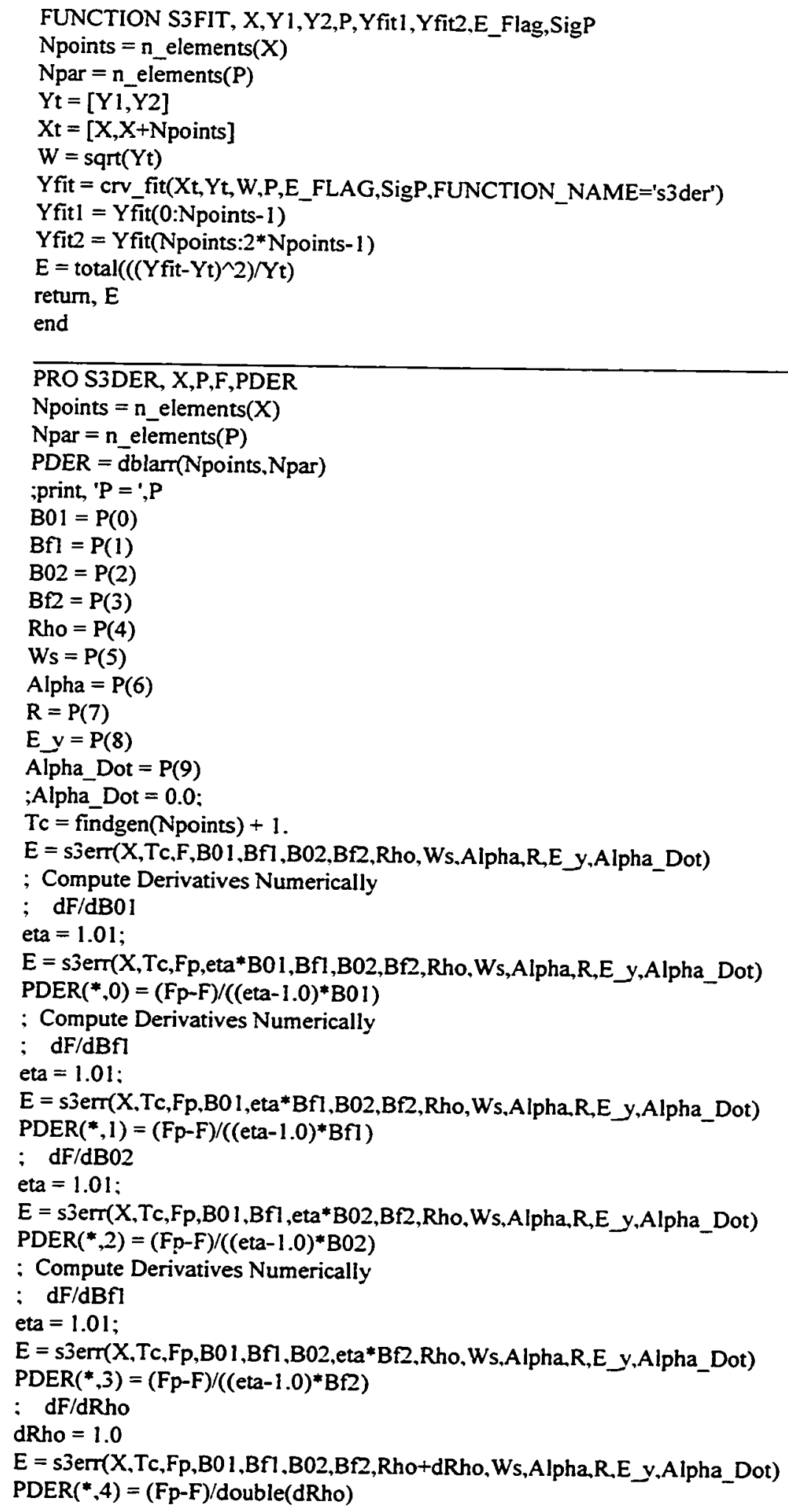




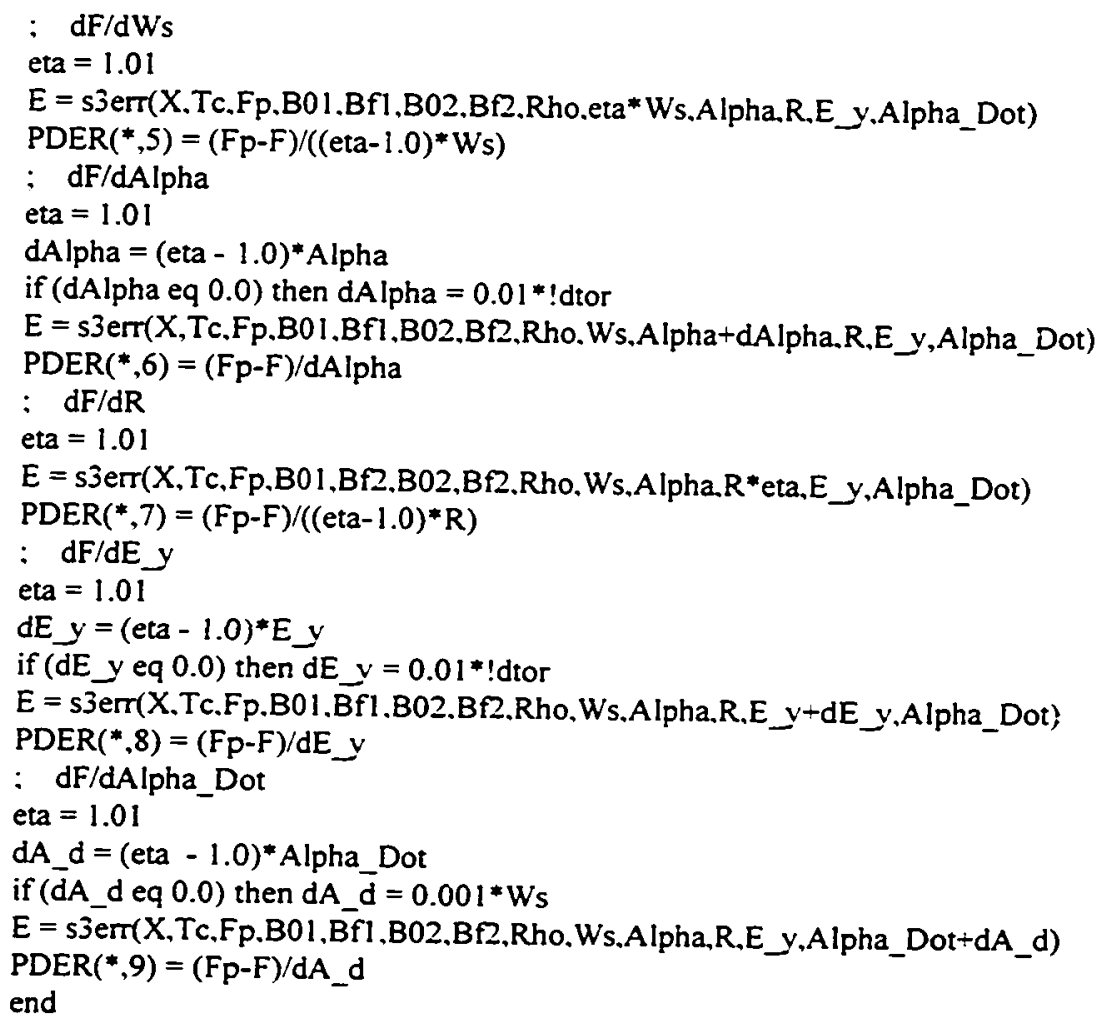




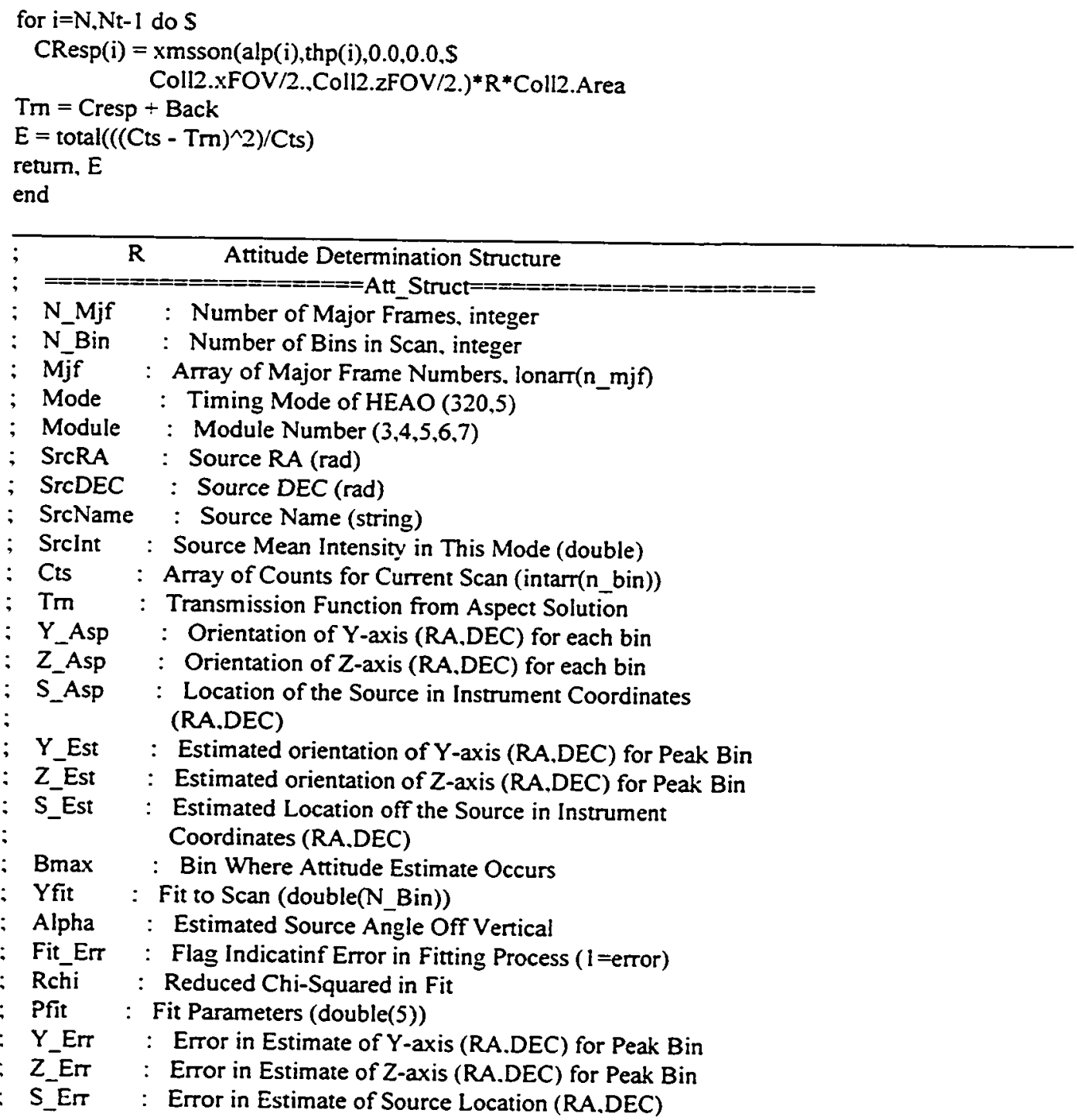




\section{Appendix H: Phase Locked Loops}

\section{H.1 Phase Detectors}

The simplest way to measure the relative phase of two signals is to use a quadrature multiplier. That is. we simply multiply the two signals together. viz.:

$$
u_{d}=u_{1}(t) u_{2}(t)
$$

Consider the case where $u_{1}$ and $u_{2}$ are sinusoidal. viz.:

$$
\begin{aligned}
& u_{1}=A_{1} \sin \left(\theta_{1}\right) \\
& u_{2}=A_{2} \cos \left(\theta_{2}\right)
\end{aligned}
$$

If the phase error, $\theta_{e}$, is defined as.

$$
\theta_{e}=\theta_{1}-\theta_{2}
$$


then the phase detector signal. $u_{d}$, can be written as follows:

$$
\begin{aligned}
& u_{d}=A_{1} A_{2} \sin \left(\theta_{1}\right) \cos \left(\theta_{1}-\theta_{e}\right) \\
& u_{d}=A_{1} A_{2} \sin \left(\theta_{1}\right)\left(\cos \left(\theta_{1}\right) \cos \left(\theta_{e}\right)+\sin \left(\theta_{1}\right) \sin \left(\theta_{e}\right)\right) \\
& u_{d}=\frac{A_{1} A_{2}}{2}\left(\sin \left(2 \theta_{1}\right) \cos \left(\theta_{e}\right)+\left(1-\cos \left(2 \theta_{1}\right)\right) \sin \left(\theta_{e}\right)\right. \\
& u_{d}=\frac{A_{1} A_{2}}{2} \sin \left(\theta_{e}\right)+\frac{A_{1} A_{2}}{2} \sin \left(2 \theta_{1}-\theta_{e}\right)
\end{aligned}
$$

The first term of $u_{d}$ is a steady term proportional to the phase error between $u_{1}$ and $\mathrm{u}_{2}$. The second term is a sine wave at twice the reference frequency. A properly designed loop filter will attenuate signals at twice the reference frequency to reduce this term. The net effect is that the phase detector signal is proportional to the phase error with a small jitter on top of it. If the phase error is small, then the phase detector can be approximated by a linear model. viz.:

$$
u_{d} \cong \frac{A_{1} A_{2}}{2} \theta_{e}=K_{p d} \theta_{e}
$$

\section{H.2 Loop Filters}

The three filters considered in this thesis are the constant gain filter. the active lag-lead filter and the proportional plus integral (PI) filter.

\section{H.2.1 Constant Gain Filter}

The differential equation governing the constant gain filter is.

$$
u_{f}=K_{f} u_{d}
$$

In the LaPlace domain this can be represented as,

$$
U_{f}(s)=K_{f} U_{d}(s)
$$




\section{H.2.2 Active Lag-Lead Filter}

The differential equation governing the active lag-lead filter is,

$$
u_{f}+\tau_{1} \dot{u}_{f}=K_{f}\left(u_{d}+\tau_{2} \dot{u}_{d}\right)
$$

In the LaPlace domain this can be represented as,

$$
U_{f}(s)=K_{f} \frac{1+\tau_{2} s}{1+\tau_{1} s} U_{d}(s)
$$

\section{H.2.3 PI Filter}

The differential equation governing the PI filter is.

$$
\tau_{1} \dot{u}_{f}=u_{d}+\tau_{2} \dot{u_{d}}
$$

In the LaPlace domain this can be represented as.

$$
U_{f}(s)=\frac{1+\tau_{2} s}{\tau_{1} s} U_{d}(s)
$$

\section{H.3 Voltage Controlled Oscillator}

The voltage controlled oscillator (VCO) is the local clock that will be used as a time reference. In this case the output of the VCO will be a sine wave. The frequency of the VCO signal is equal to the sum of a constant frequency. called the center frequency. and an offset frequency that is proportional to the input. viz.:

$$
\omega_{v c o}(t)=\omega_{0}+K_{v c o} u_{f}(t)
$$

The phase of the VCO $\left(\theta_{2}\right)$, is just the integral of $\omega_{\text {veo }}$,

$$
\theta_{2}(t)=\int_{0}^{t} \omega_{v c o}(t) d t+\theta_{2}(0)
$$


This can be written in the LaPlace domain as.

$$
\Theta_{2}(s)=\frac{K_{v c c}}{s} U_{f}(s)
$$

The output of the VCO introduces a nonlinearity into the system. viz.:

$$
u_{2}(t)=\cos \left(\theta_{2}(t)\right)
$$

\section{H.4 General Linearized Equations for the PLL}

By using the linear model of the phase detector (H.1.6), a general transfer function for the PLL in the locked state can be derived, viz:

$$
\begin{aligned}
& H(s)=\frac{\Theta_{2}(s)}{\Theta_{1}(s)}=\frac{K_{v c o} K_{p d} F_{l f}(s)}{s+K_{v c o} K_{p d} F(s)} F_{p f}(s) \\
& H_{e}(s)=\frac{\Theta_{e}(s)}{\Theta_{1}(s)}=\frac{s}{s+K_{v c o} K_{p} F_{l f}(s)} F_{p f}(s)
\end{aligned}
$$

\section{H.5 Simulation Prefilter - Butterworth Bandpass Design}

Using the MATLAB rountine 'BUTTER', a butterworth bandpass filter was designed for the $\mathrm{x}$-ray timing simulation. The state-space representation of this filter is given in equation H.5.1.

$$
\begin{aligned}
\Phi_{p f} & =\left[\begin{array}{cccc}
5.1789 e-1 & -4.7702 e-2 & 7.7451 e-1 & -2.4340 e-2 \\
4.7702 e-2 & 5.8535 e-1 & 2.4340 e-2 & 8.0893 e-1 \\
-7.7451 e-1 & 2.4340 e-2 & 6.0481 e-1 & 1.2419 e-1 \\
-2.4340 e-2 & -8.0893 e-1 & -1.2419 e-2 & 5.8724 e-1
\end{array}\right] \quad \Gamma_{p f}=\left[\begin{array}{c}
85024 e-2 \\
2.6720 e-3 \\
-4.3384 e-2 \\
-1.3634 e-3
\end{array}\right] \\
H_{p f} & =\left[\begin{array}{llll}
1.6865 e-2 & 5.6051 e-1 & 8.6054 e-3 & 2.8600 e-1
\end{array}\right] \quad J_{p f}=\left[\begin{array}{l}
9.4469 e-4
\end{array}\right]
\end{aligned}
$$


The frequency response of this filter is shown in Figure H.1.
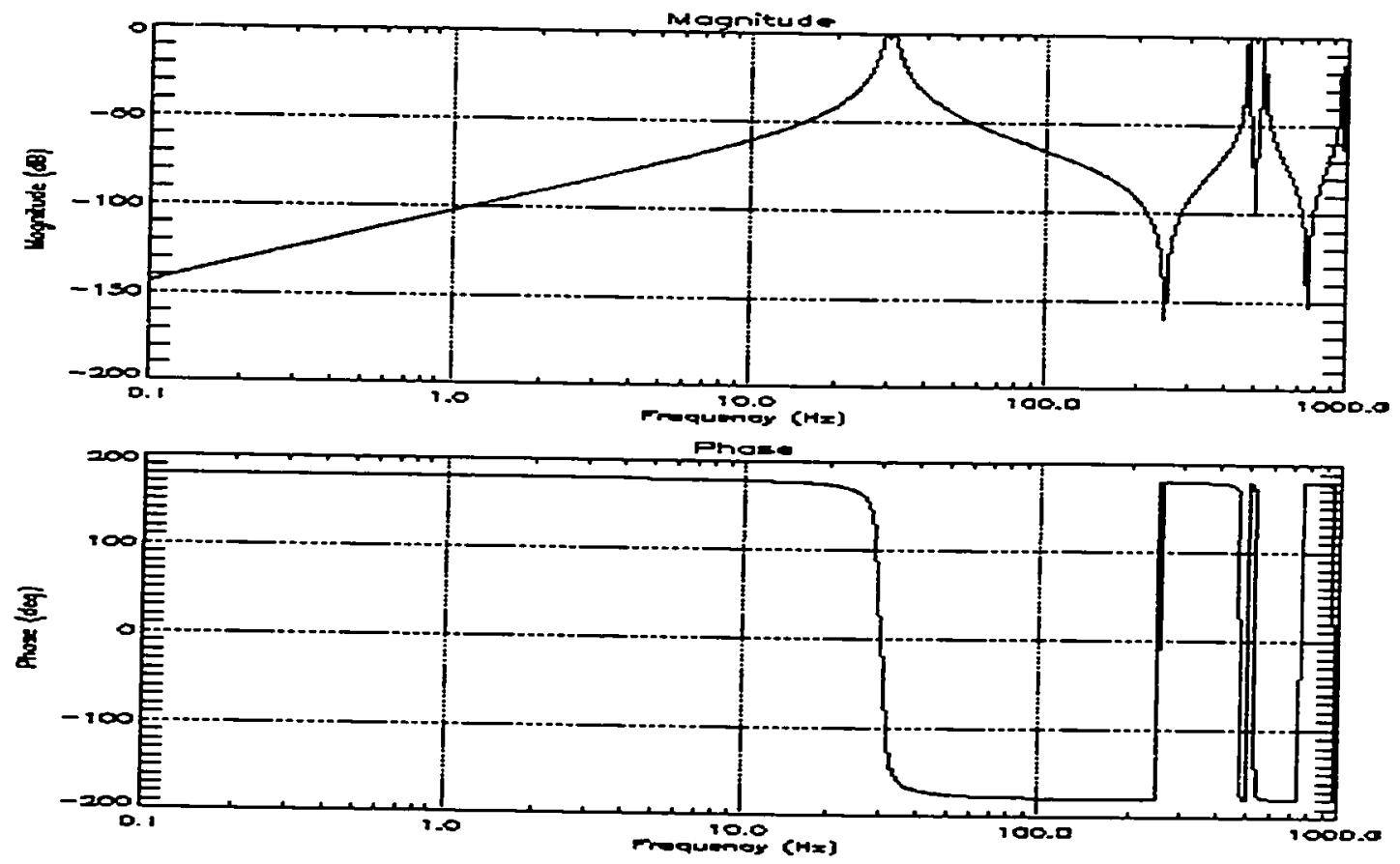

Figure H.1: Prefilter Frequency Response 


\section{Appendix I: Phase Locked Loop Simulation - IDL Procedures}

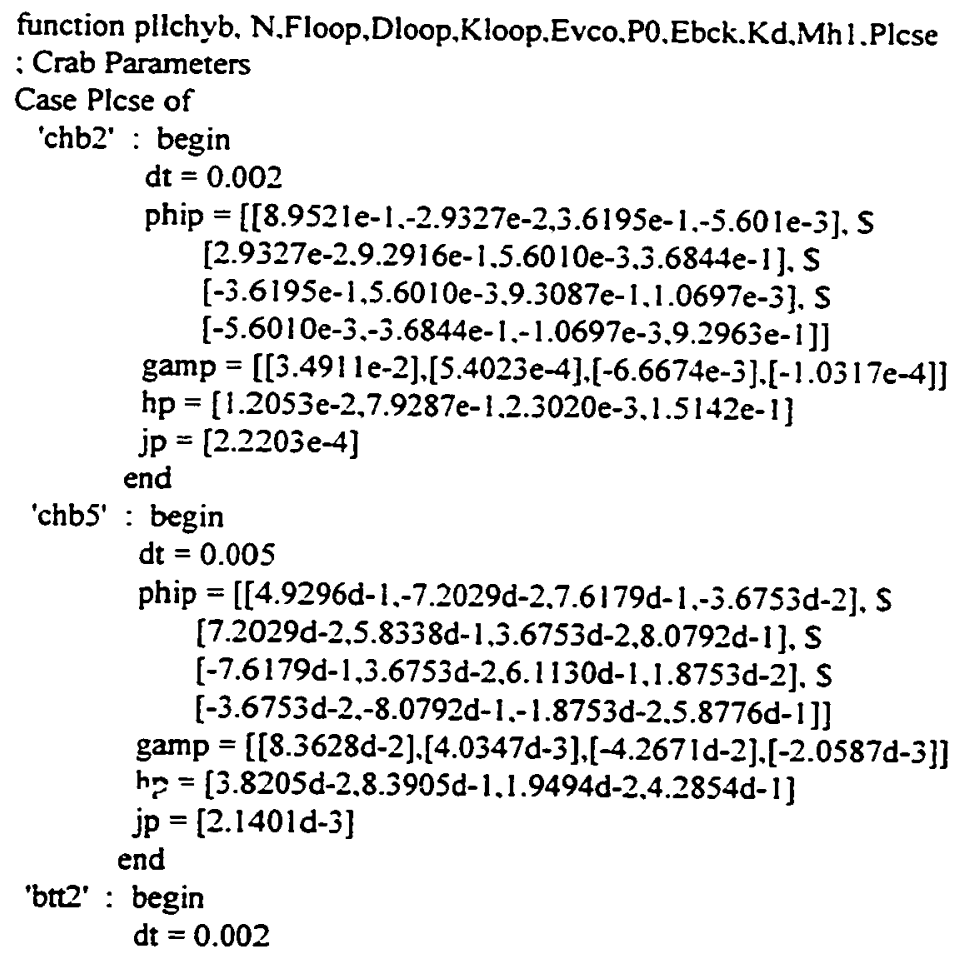




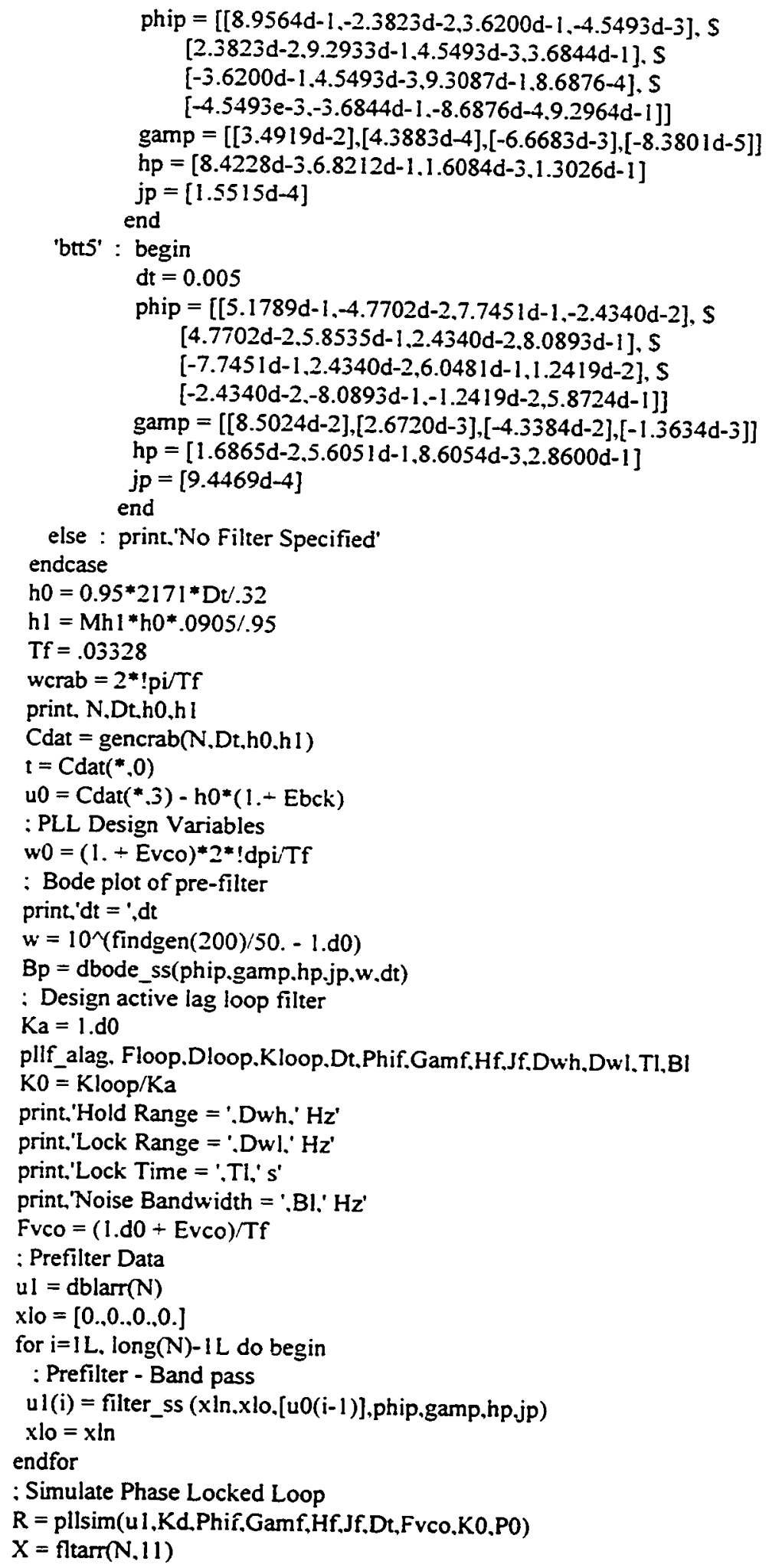




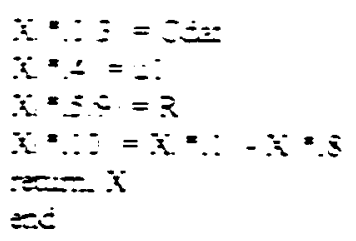

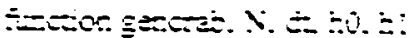

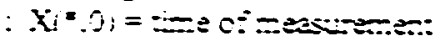

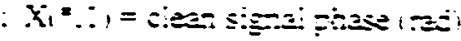

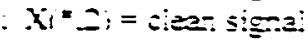

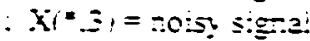

$x=0$

$x_{i}=0,=$ indgen! $>i=$

: Fill Cas Aray

$\mathrm{Nci}=10001$

$c e=$ ticrabrici.eno.til

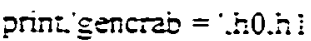

: Interpolate to Find Count Rate

$i=x(* 0)-\operatorname{long}\left(x t^{*} .01 \text {.03:32 }\right)^{*} .03528$

$t=i^{*}+\mathrm{ici} .03328$

$i l=\operatorname{lon} g(t)$

$2=\mathrm{Il}-1 \mathrm{~L}$

$x(* 2)=\operatorname{cc}(t))-(\operatorname{cc}(2)-\operatorname{cc}(t 1))=(t-t 1)$

$x(* .1)=x(* .0)=2$. !dpi .03328

$X(* . j)=\operatorname{poidev}(X(* .2))$

return. $x$

end

pro PLLF.ALAG. Fn.Damp.K.Dt.Phi.Gam.H.J.Dwh.Dw.TI.B

wn $=2 . \mathrm{d} 0 *: \mathrm{dpi} * \mathrm{Fn}$

$\mathrm{Ka}=1.0$

tau2 $=2 *$ Damp un $-1 . \mathrm{K}$

taul $=\mathrm{K} / \mathrm{wn} 2$

print.taul.tau2.damp.wn

if ((taul gt 0.0$)$ and (tau2 gt 0.0$)$ ) then begin clss_alag. Ka.taul.tau2.A.B.C.D

c2d_ss. A.B.C.D.Phi.Gam.H.J.Dt.'prewarp',wn

endif else $S$

print.'Improper Loop Specifications'

: Compute Loop Performance Paramters

Dwh $=K /(2 . \mathrm{d} 0 *$ !dpi)

$\mathrm{Dwl}=\mathrm{K}^{*}(\operatorname{tau} 2 / \operatorname{tau} \mathrm{I}) /\left(2 . \mathrm{d} 0^{*} ! \mathrm{dpi}\right)$

$\mathrm{Tl}=1 . \mathrm{d} 0 / \mathrm{Fn}$

$\mathrm{Bl}=(\mathrm{Fn} / 2 . \mathrm{d} 0)^{*}(\mathrm{Damp}+0.25 / \mathrm{Damp})$

end

function filter_ss, x.x0,u,phi.gam.h.j

; $x(k+1)=p h i^{*} x(k)+g a m^{*} u(k)$

; $y(k)=h^{*} x(k)+j * u(k)$

$x=x 0$ \#phi + u\#gam

$y=x \neq h+u \# j$

return. $y$ 


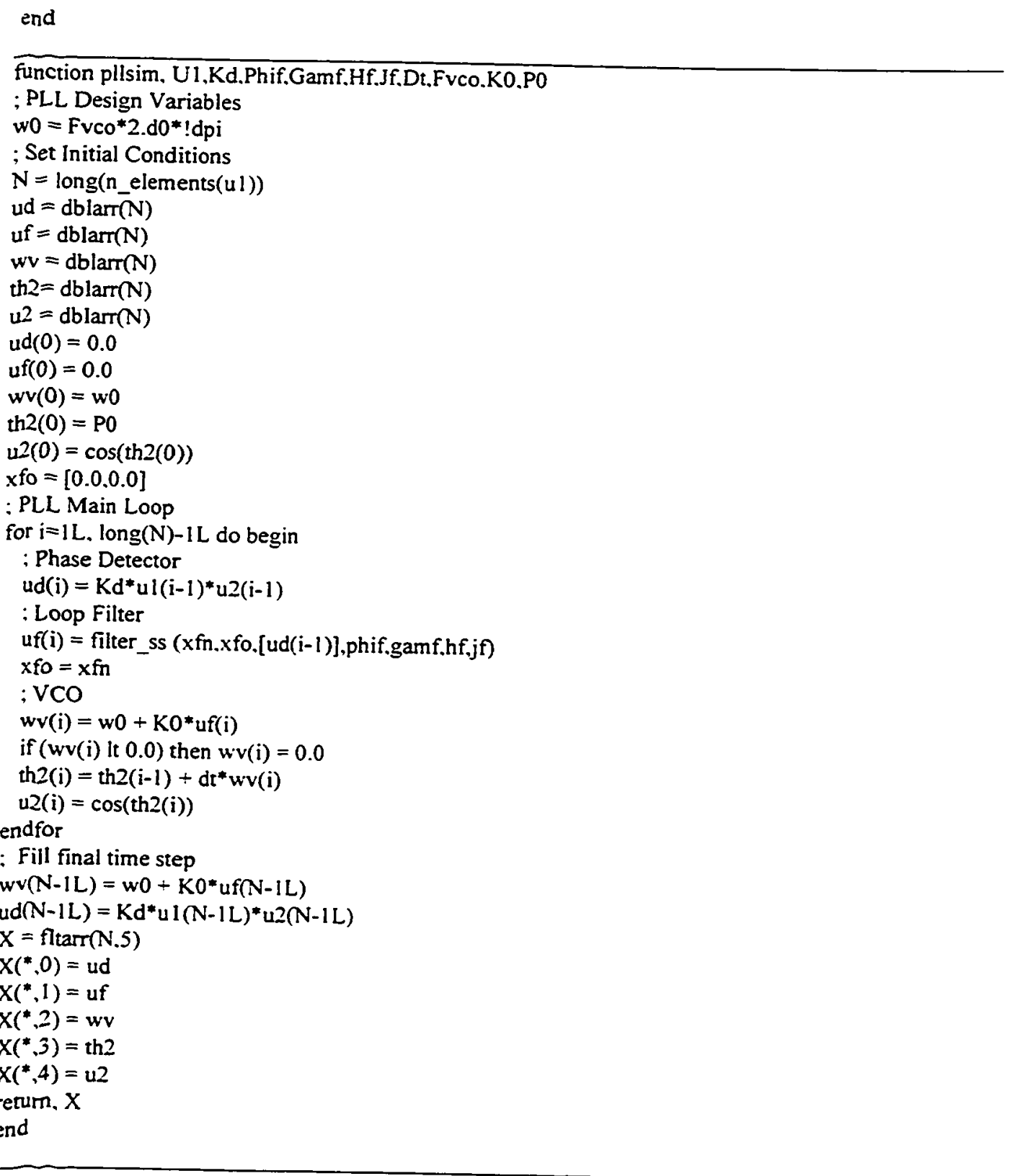




\section{References}

Aluminum Association. Aluminum Brazing Handbook, 4th ed. Washington. D.C.: Aluminum Association. 1990.

Best. Roland E. Phase Locked Loops: Theory, Design and Applications. New York: McGraw Hill. Inc., 1993.

Bevington. Philip R. and D. Keith Robinson. Data Reduction and Error Analysis for the Physical Sciences. New York: McGraw Hill. Inc., 1992.

Black, Harold D. "Early Development of Transit. The Navy Navigation Satellite System." Journal of Guidance, Navigation and Control. 13:577-585, 1990.

Blanchard. Alain. Phase-Locked Loops: Appiication to Coherent Receiver Design. New York: John Wiley \& Sons, Inc.. 1976.

Bradt. Hale V.D.. et al. "X-ray Astronomy Missions". Annual Review of Astronomy and Astrophvsics. 30:391-427, 1992.

Byard. Kevin. “On Self-Supporting Coded Aperture Arrays.” Nuclear Instruments and Methods in Physics Research. A322:97-100. 1992.

Cohen. Clark E. et al. "Space Flight Tests of Attitude Determination Using GPS." Intemational Joumal of Satellite Communications. 12:427-33. 1994.

Crerie. Jeffrey: Phase-Lock roll Control of Inertially Pointing Spacecraft. Unpublished $\mathrm{PhD}$ Thesis. Stanford University. Stanford. Ca.. 1993.

Dicke. R.H. "Scatter-Hole Cameras for X-rays and Gamma Rays." Astrophysical Journal. 153:Li 101-L106. 1968.

Fabian. A.C. and X Barcons. "The Origin of the X-ray Background". Annual Review of Astronemv and Astrophysics. 30:429-456. 1992.

Fenimore. E. E. and T. M. Cannon. "Coded Aperture Imaging with Uniformly Redundant Arrays." Applied Optics. 17:337-347, 1978.

Forman, W. et al. "The Fourth Uhuru Catalog of X-ray Sources." The Astrophysical Joumal Supplement Series. 38:357-412, 1978.

Gardner, Floyd M. Phaselock Techniques. New York: John Wiley \& Sons, 1966.

Gardner. Floyd M. Phaselock Techniques. New York: Jchn Wiley \& Sons. 1979. 
Hammersley, Andrew et al. "Reconstruction of Images from a Coded-Aperture Box Camera." Nuclear Instruments and Methods in Physics Research. A31 1:585-594. 1992.

Hanson. John E. "USA Structure Test Program." Internal report. Astrogravity Note \#17. Stanford Linear Accelerator Center, Stanford. Ca.

Hanson, John E. "USA Dip Brazing Manufacturing Qualification." Internal report. Astrogravity Note \#18. Stanford Linear Accelerator Center. Stanford. Ca.

Hanson. John E. "USA Structure Quality Assurance Testing." Internal report. Astrogravity Note \#19. Stanford Linear Accelerator Center. Stanford. Ca.

Hanson. John E. "USA Pylon Static Load Test." Internal report. Astrogravity Note \#20. Stanford Linear Accelerator Center, Stanford. $\mathrm{Ca}$.

Harnden. F. R. and F.D. Seward. "Einstein Observations and the Crab Nebula Pulsar." The Astrophysical Journal. 283:279-285, 1984.

Hoech. Robert. et al. "Design. Capabilities and Performance of a Miniaturized Airborne GPS Receiver for Space Applications." Paper presented at IEEE 1994 Position Location and Navigation Symposium. New York. NY. 1994.

Kau, S.P. et al. "Autonomous Satellite Orbit Navigation Using Known and Unknown Earth Landmarks." Paper presented at AIAA Guidance and Control Conference. Boston. Mass. Aug 20-22. 1975.

Massen, Robert. “Sensors with Coded Apertures." Journal of Physics E: Scientific Instruments. 20:595-600. 1987.

The Math Works. Inc. Control Svstem Toolbox Fer Use with MATLAB. Natick. Mass.: The Math Works. Inc. 1992.

The Math Works. Inc. MATLAB Reference Guide. Natick. Ma.: The Math Works. Inc. 1992.

Mendel, Jerry M. Maximum Likelihood Deconvolution. New York: Springer-Verlag. 1990.

Ponman, Trevor et al. "Error Analysis for a Noncyclic Imaging System." Nuclear Instruments and Methods in Physics Research. A262:419-429, 1987.

Research Systems. Inc. IDL User's Guide. Boulder. Co.: Research Systems, Inc. 1994. 
Research Systems, Inc. IDL Reference Guide. Boulder, Co.: Research Systems. Inc. 1994.

Skinner, G.K. "Imaging with Coded-Aperture Masks." Nuclear Instruments and Methods in Physics Research. A221:33-40, 1984.

Staff of the Space Department. Johns Hopkins University APL, and Staff of the Guidance and Control Laboratory, Stanford University. "A Satellite Freed of all but Gravitational Forces: 'TRIAD I'." Joumal of Spacecraft and Rockets. 11: 637644, 1974.

Stanley, William D.. et al. Digital Signal Processing. Reston, Va.: Reston Publishing Co.. Inc., 1984.

Taylor. Fred J. Digital Filter Design Handbook. New York: Marcel Dekker. Inc.. 1983.

Van Bezooijen, Roelof W.H. "Star Sensors for Autonomous Attitude Control and Navigation." Optical Technologies in Aerospace Sensing, 1992.

Vessot, Robert F.C. "Experimental Techniques with Highly Stable Clocks." Paper presented at XIVth Moriond Workshop on Dark Matters in Cosmology. Clocks and tests of Fundamental Laws. Villars-Sur-Ollon, Switzerland. January 21. 1995.

Wen. Han. "Determining Dead Time." Internal report. Astrogravity Note \#14. Stanford Linear Accelerator Center. Stanford. Ca.

Wertz. James R.. ed. Spacecraft Attitude Determination and Control. Boston. Ma: Kluwer Academic Publishers, 1978.

White. Robert L. et al. "Autonomous Satellite Navigation Using Observations of Starlight Atmospheric Refraction." Navigation. 32:317-333. 1985-86.

White. R. L. et al. "Use of Known Landmarks for Satellite Navigation" Paper presented at AIAA Guidance and Control Conference. Boston. Mass. Aug 20-22, 1975.

White. N.E. et al. "The Properties of X-ray Binaries." In X-rav Binaries, edited by Walter H.G. Lewin et al. Cambridge, UK: Cambridge University Press, 1995:157.

Wood. K.S. et al. "The HEAO A-1 X-ray Source Catalog". The Astrophysical Joumal Supplement Series. 56: 507-649, 1984.

Wood. K.S. "Navigation Studies Utilizing the NRL-801 Experiment and the ARGOS Satellite." Preprint. 1993. 